\title{
PROCEEDINGS
}

\section{Workshop on the Measurement of Transient Pressure and Temperature}

\section{3-24 April 1991}

\section{Editors}

Vern E. Bean

Gregory 」. Rosasco

U.S. DEPARTMENT OF COMMERCE Technology Administration National Institute of Standards and Technology Process Measurement Division Gaithersburg, MD 20899 



\section{PROCEEDINGS}

\section{Workshop on the Measurement of Transient Pressure and Temperature}

\section{3-24 April 1991}

\section{Editors}

Vern E. Bean

Gregory J. Rosasco

U.S. DEPARTMENT OF COMMERCE Technology Administration

National Institute of Standards and Technology

Process Measurement Division

Gaithersburg, MD 20899

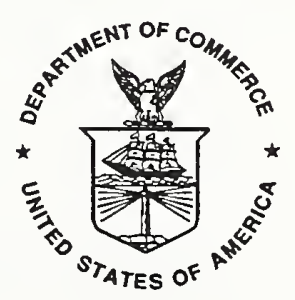

U.S. DEPARTMENT OF COMMERCE Barbara Hackman Franklin, Secretary

TECHNOLOGY ADMINISTRATION Robert M. White, Under Secretary for Technology

NATIONAL INSTITUTE OF STANDARDS

AND TECHNOLOGY

John W. Lyons, Director 


\begin{abstract}
Workshop on the
Measurement of Transient Pressure and Temperature
\end{abstract}

$$
\text { 23-24 April } 1991
$$

National Institute of Standards and Technology Gaithersburg, Maryland

Page

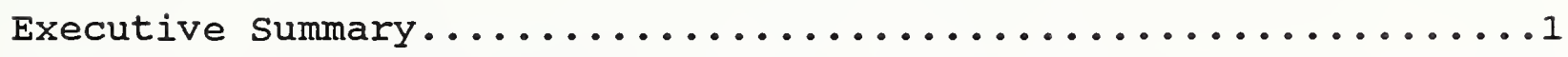

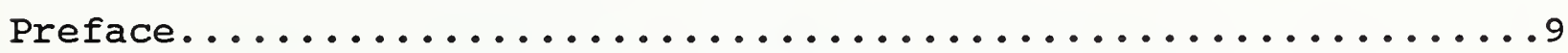

(Speakers are indicated by bold type.)

Dynamic High Pressure Measurement: Issues and Instrumentation

Marek I. Winiarz, G. E. Aircraft Engines, Cincinnati, Ohio......10

Tourmaline Gage for Navy's Underwater Explosion Shock

Measurements

Ronald B. Tussing, Naval Surface Warfare Center, White Oak,

Silver Spring, Maryland.......................... 8

Dynamic Pressure Requirements of the Army

John Ball, Redstone Arsenal, Alabama.................69

The Search for a Transient Pressure Standard: A Collaborative Effort for NASA and NIST

Troy J. Estes, NASA, White Stands, Las Cruces, New Mexico,

william C. Smith, Lockheed Engineering and Sciences

Center, White Sands, Las Cruces, New Mexico.................78

Fast Pressure Measurements: The Need for PVDF Calibration

Standards

Bruce Hartenbaum, H-Tech Laboratories, Santa Monica,

California,

Bradford Sturtevant, California Institute of Technology,

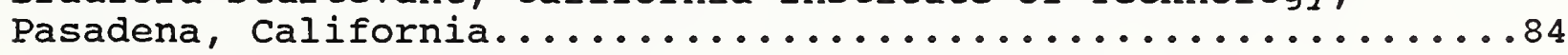

Dynamic Step-Pressure Calibration

James F. Lally, PCB Piezotronics, Depew, New york...........104

Improved Accuracy in Piezoelectric Measurements Using a Low

Noise, Ultra-High Isolation Signal Conditioner

Bruce Hartenbaum, H-Tech Laboratories, Santa Monica,

California,

James Morrison, Lawrence Livermore National Laboratory,

Livermore, California,

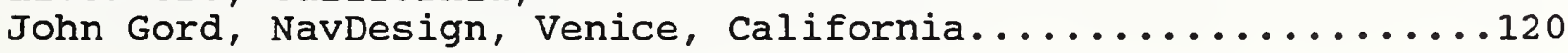


Three Case Studies of Strain/Test-Induced Voltages Which Obscured Signals

Peter R. Stein, Stein Engineering Services, Inc., Phoenix,

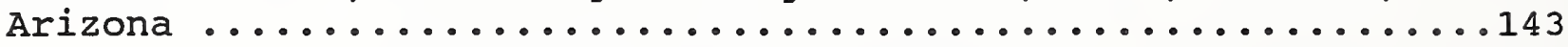

Calibration of Ballistic Pressure Transducers Arpad A. Juhasz and Charles D. Bullock, Aberdeen Proving Ground, Maryland,

Don Newhall, Harwood Engineering, Walpole, Massachusetts......146

Dynamic Pressure Calibrator

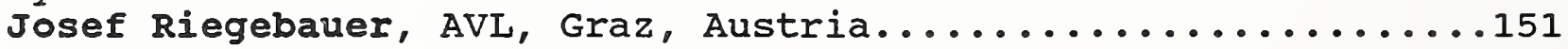

Means of Dynamic Calibration for Pressure Transducers

J. P. Damion, Ecole Nationale Superieure d'Arts et Metiers,

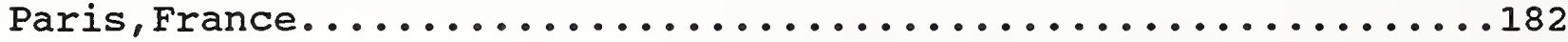

A Proposed Dynamic Pressure and Temperature Primary Standard Gregory J. Rosasco, Vern E. Bean, and Wilbur S. Hurst, NIST,

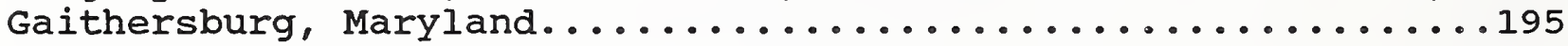

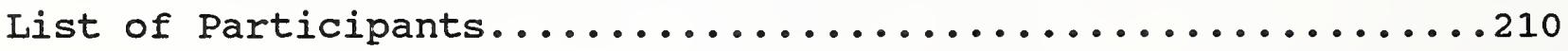




\section{EXECUTIVE SUMMARY}

The Workshop on the Measurement of Transient Pressure and Temperature was held at the National Institute of standards and Technology (NIST), Gaithersburg, MD, on 23-24 April 1991. There were 77 attendees, 55\% from government and 45\% from the private sector. Aerospace, automotive, and instrumentation manufacturers were present along with engineers from private sector and government (Army, Navy, Air Force, and NASA) research organizations. The Army, Navy, and NASA primary standards laboratories also were represented. The workshop drew international attention with participants from Taiwan, Sweden, Austria, and France. Only France currently offers transient pressure calibrations as part of their national measurement system.

The purpose of the workshop was to identify current and emerging measurement problems and needs for transient pressure and temperature measurements, to explore scientific and technical barriers and opportunities for achieving measurement accuracy goals, and to assist in the formulation of an appropriate role for NIST.

The scope of this workshop was the measurement of transient pressure and transient temperature; however, the participants were much more concerned with pressure than with temperature. Temperature was discussed briefly by two speakers: Damion described the use of a shock tube to provide a transient temperature stimulus to study a special platinum resistance thermometer and Rosasco described how spectroscopic techniques can be used to measure both transient temperature and pressure.

\section{Measurement Problems and Needs}

The range of industrial and scientific interests represented at the workshop is a reflection of the importance of transient measurements in a wide range of applications, such as aeronautics, blast-wave analysis, internal combustion and jet engine testing, ballistics, health, and safety (see for example winiarz, Ball, Estes and Smith, Hartenbaum and sturtevant). The accuracy of these transient measurements is crucial, and a common thread in many of the presentations was the need for traceability to national standards at NIST. In many cases, agency policy and government regulations require such traceability (Ball, Estes and Smith). There currently are no national primary standards for the measurement of either transient pressure or transient temperature in the metrology system of the United states, and hence there is no traceability to NIST.

As one might expect from the range of applications, the amplitude and frequency of transient pressure calibration needs are widely varied and range, for example, from $13 \mathrm{~Pa}$ at $50 \mathrm{kHz}$ for the national aerospace plane to $1 \mathrm{GPa}$ at $100 \mathrm{MHz}$ for studies on explosives. Estes and smith documented the requirements of NASA. We present these in Fig. 1 as an outline in pressure and frequency 
space, with the numbers inside the outline being the desired accuracies in that pressure-frequency region, expressed as percents. At the conclusion of the workshop, the participants were invited to indicate the pressure and frequency ranges of the calibration services they needed with the corresponding accuracies. Figure 2 is a collage of the replies from organizations other than NASA. Again, the numbers inside the outline represent the needed accuracies expressed as percents.

\section{Current Practice in Transient Pressure Calibrations}

Because there is no traceability to NIST for transient pressure or temperature measurements, laboratories that need to make such measurements are left to their own resources to develop calibration and testing programs. Calibration generally is understood to mean "... a test during which known values of measurand are applied to a transducer and corresponding output readings recorded" (see Ially who quotes $A$ Guide for the Dynamic Calibration of Pressure Transducers, ANSI MC88.1-1972, published by The American Society of Mechanical Engineers, New York, NY). Because of the technical problems associated with providing a known measurand for transient pressure, an approach often taken is to assume a calibration with static pressure standards and methods to be valid for pressure transducers used under transient pressure conditions. As widely documented by the speakers, this assumption is incorrect because the pressure transducer is a mechanical structure which will behave differently at different frequencies. The static calibration yields no frequency behavior information.

The ranges of the needed calibration services indicated in Figs. 1 and 2 span more than eight orders of magnitude in frequency and six orders of magnitude in pressure. It is not surprising that multiple techniques are required to meet the needs. The current state-of-the-art in transient pressure calibrations was presented by a number of speakers. From the discussions, we observe that as the frequency and pressure increase, the difficulty of generating a known measurand for the calibration of transducers also increases.

A variety of transient pressure generators were discussed (Lally, Newhall, Riegebauer, Damion) including the pistonphone, dead weight tester with a quick-opening valve, pneumatic and hydraulic quickopening valve devices, a column of liquid mounted on a shaker head, a modified air compressor, a weight falling on a piston which compresses a confined liquid (drop-hammer), and the shock tube. For the very high frequency regime, i.e. for nanosecond rise-time devices, Hartenbaum and sturtevant suggested the use of an electrically driven flyer plate to produce the requisite (i.e. in frequency and amplitude) shock waves.

These generators may be classified as periodic or aperiodic. Periodic generators yield sinusoidally varying pressures, are limited to lower frequencies, and suffer decreased amplitude as the frequency increases. The pressure amplitudes are generally not 
known by absolute means and thus periodic generators are used as comparators rather than as primary standards (Damion). The quickopening valves, the drop hammer, and the shock tube are aperiodic generators and were considered in greater detail.

Aperiodic generators yield a pressure step or a pressure impulse. These generators subject the transducer to a range of frequencies, the high frequency limit of the generator usually depends upon the rise time of the pressure; the low frequency limit depends upon the duration or width of the pressure stimulus. The description of the complete frequency content of the stimulus depends on the details of the temporal evolution of the step/impulse which, in general, are not known. We observe from many of the presentations that it is difficult to assess the sources of the high frequency content in the response of a transducer under calibration. Some oscillations observed with a quick-opening valve step-generator are thought to be a common characteristic of such devices (Newhall). Defective transducers or defects in the electrical cables and connections also can give rise to strong oscillations in the step or impulse response (Lally, Newhall). Shock waves also have high frequency content which is not well understood. Under these conditions, the frequency information derived from the response of a transducer used with such generators is essentially qualitative.

The steady-state amplitudes for the step generator, or peak amplitudes for the impulse generator, are used to determine the transducer sensitivity (the ratio of the transducer output voltage to unit pressure). These amplitudes are derived either from direct measurement of steady-state pressures in quick-opening valve devices (Newhall, Lally, Damion) or from calculations, for example, ideal shock-wave theory (Lally, Damion) or a quasi-empirical model for the drop-hammer generator (Riegebauer). In the latter paper, two different approaches are used to arrive at the peak pressure and good agreement, within $4 \%$, is demonstrated.

France is the only nation currently offering transient pressure calibration services as part of their national measurement system. The laboratory is located at Ecole Nationale supérieure d'Arts et Metiers (ENSAM) in Paris and is under the direction of $J . P$. Damion. The service covers the frequency range from a few $\mathrm{Hz}$ to a few tens of $\mathrm{kHz}$ at pressures up to $20 \mathrm{MPa}$. Quick-opening valve devices are used for the lower frequencies and shock tubes for the higher frequencies (Damion). Damion's discussion gives good account of the practical limits and assumptions implicit in the use of the various calibration devices.

\section{Pressure Transducers}

Many of the presentations and the discussions following them indicated that modern transducer technology, based on a wide variety of physical phenomena, is highly developed. Transducer resonance frequencies greater than $100 \mathrm{kHz}$ are routinely achieved, and thus measurements for frequencies up to 10's of kilohertz can be made. Lally presented sensitivity data for a transducer 
obtained by 5 different techniques: the dead-weight tester, a hydraulic drop-hammer, two varieties of quick-opening valve devices, and a shock-tube. Excellent consistency, at the 2\% level, was demonstrated for the sensitivity of this transducer, which has a resonance frequency in the range of $500 \mathrm{kHz}$. Newhall compared a piezoelectric gage and a strain-type gage subjected to a $700 \mathrm{MPa}$ pressure step in a quick-opening valve device and reported virtually identical responses. Riegebauer.demonstrated transducer repeatability at the $0.5 \%$ level for a drop-hammer device with a rise time in the 1-2 ms regime. Perhaps the most widely tested transducers are the Navy's tourmaline-based piezoelectric devices.

The Navy's underwater explosion shock measurements program has used gages made of single-crystal tourmaline for over 40 years. Tourmaline is bulk or hydrostatically sensitive. It does not require any mechanical shielding to permit only a certain direction of strain as does quartz, thus avoiding the complexities of diaphragms, mechanical resonances, and reflections. The Navy calibrates the gage statically by means of a dead weight tester and assesses the time response with a quick-opening valve. The gage calibration constant is stable and linear over the span of 0.1 to $350 \mathrm{MPa}$. Many gages used and recalibrated over a 15 year period show no aging effects. The gage shows no signs of hysteresis. The tourmaline gage remains the Navy's gage of choice (Tussing).

\section{Signal Transfer and Processing}

Modern high speed digital data recording systems are routinely used for transient measurements, and indications are that they are quite adequate, in terms of speed, accuracy, and data processing capability, for the needs of the community. In any application there are important issues with respect to spurious signal generation and pick-up and with the noise associated with the measurement environment; these issues were addressed by many authors (Winiarz; Ball; Hartenbaum, Morrison and Gord; Stein). such signal/noise problems are ubiquitous. Some are associated with the transducer, for example, the effects of temperature transients are familiar. Stein also cautioned us to be wary of strain-induced voltages in impedance-based measurement devices. other problems, perhaps the most pervasive, are associated with the transfer of the electrical signal to the recording device. Hartenbaum et al. described advances in noise reduction by means of a new signal conditioner based on hybrid optical-electronic circuitry. These types of problems must be considered in any experimental design, including that of a calibration facility. In general, care must be exercised in analyzing and interpreting the frequency content of one's signal, especially the high frequency components.

\section{v. Technical Opportunities and Barriers}

In assessing the current state of the national calibration system for transient pressure, we note first that there are dozens of laboratories active in this area. Most of these have transient 
sources which are used mainly for qualitative assessment of transducer response functions, e.g., no unexpected resonance behavior, lag, overshoot, or decay. Some laboratories have transient sources where the amplitude of the step stimulus or of the peak impulse stimulus is known.

We also observe that transient pressure transducer technology is quite reliable. This implies that, once calibrated, transducers can be used to transfer national standards to calibration laboratories and to maintain the accuracy of a national calibration system. These observations provide a technical basis for establishing a national calibration system in order to provide the needed traceability for transient pressure measurements.

At the present time, no transient generator is sufficiently well understood to allow it to serve as a primary standard. Assurance of the accuracy of our knowledge of the pressure-amplitude versus frequency for a transient source becomes increasingly difficult for frequencies above $1 \mathrm{kHz}$. The pressure wave in a shock tube (the highest frequency source for gas phase calibrations) can only be assumed to follow ideal shock wave theory. Detailed information on the rise time, shock front curvature, shock attenuation, or other non-ideal behavior, although of concern, has not yet been obtained for calibration sources (Damion). At the present time we cannot resolve this issue by recourse to a first principles theory for the transient phenomenon. Furthermore, because of the high frequency content, conventional transducers cannot be used to answer the questions experimentally. This situation limits the ability to characterize, even qualitatively, the transducer response function above a few 10's of $\mathrm{kHz}$.

These observations underlie the approach NIST is following to produce a primary standard for the measurement of transient pressure and temperature (Rosasco). In the NIST approach, the primary standard is the pressure and temperature sensor, a sample of diatomic gas which is part of the pressurizing gas. Diatomic gas molecules have a fundamental vibrational motion whose frequency is a function of pressure. These molecules also have well defined rotational energy levels whose populations provide a reliable measure of temperature. The vibrational frequencies and the populations of the rotational energy levels can be determined by laser spectroscopy. The pressure standard can ultimately be regarded as primary in that the shift and widths of the spectral features used to determine the pressure can be obtained from $a b$ initio theory. The temperature measurement approach is also primary in that it is based on the Boltzmann equation for the thermodynamic system in local equilibrium.

The gas molecule transducer offers significant advantages over conventional transducers. It does not contaminate the pressure information through resonance, phase lag, ringing, damping, limited frequency response, and limited rise time as is the case with conventional transducers. The signals carrying the pressure and temperature information are transferred to the recording device by 
means of laser beams; the traditional sources of noise and pick-up are thereby eliminated. Significantly, this transducer provides simultaneous pressure and temperature information which is necessary to completely specify a transient source.

In order to be useful for calibration of conventional transducers, the proposed primary standard needs to be realized in a transient source. The source to be used is a shock tube capable of generating reflected shock pressures up to $20 \mathrm{MPa}$. The shock tube will be calibrated by means of the molecular transducer to avoid the necessity of assuming ideal shock wave behavior for calculating the pressure and temperature from shock tube theory. The calibrated shock tube will serve as a reference standard for the calibration of conventional pressure transducers and will provide a method for decoupling pressure and temperature effects in the transducer response.

\section{Suggestions for the Role of NIST}

There was considerable discussion on the role of NIST in solving these measurement problems. The consensus view which emerged from the discussions can be summarized as follows:

1. NIST should develop standards to provide traceability for the calibration of transient pressure transducers.

2. The participants were generally willing to collaborate on these measurement problems through a working group organized to provide a forum for discussion, and through measurement roundrobins for transient pressure. More than 15 laboratories indicated a willingness to be involved in such round-robins.

3. The shock tube is the instrument of choice for obtaining pressure transducer transfer functions because of the fast rise time of the pressure step.

4. There was genuine interest in the proposal by NIST to develop a national standard based on the transfer of a molecular-level primary standard to a reference shock tube.

V. E. Bean

G. J. Rosasco 


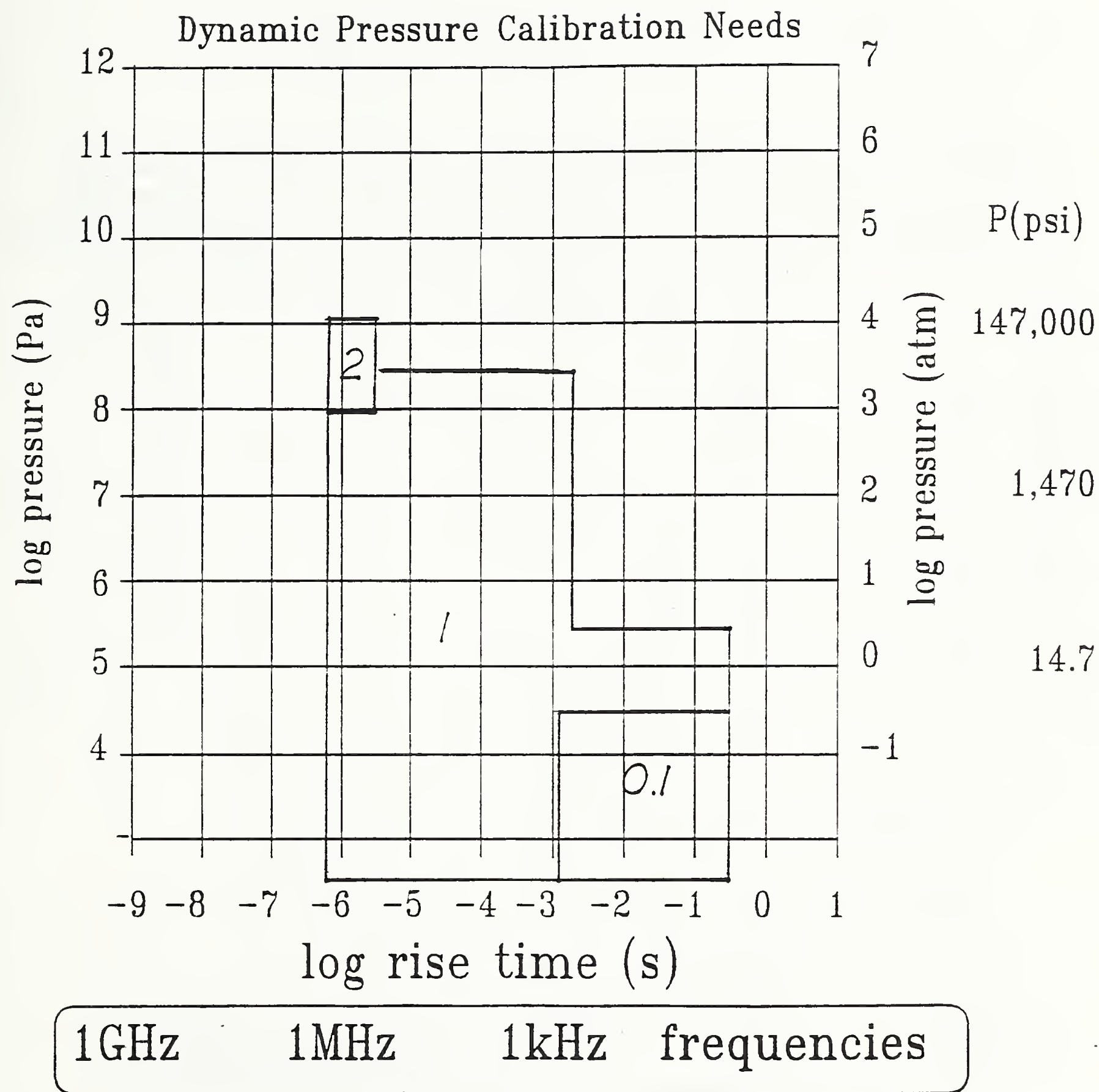

Figure 1. The outlined areas in pressure-frequency space represent the transient pressure calibration needs of NASA. The numbers in the areas represent the needed accuracies expressed in percent. 


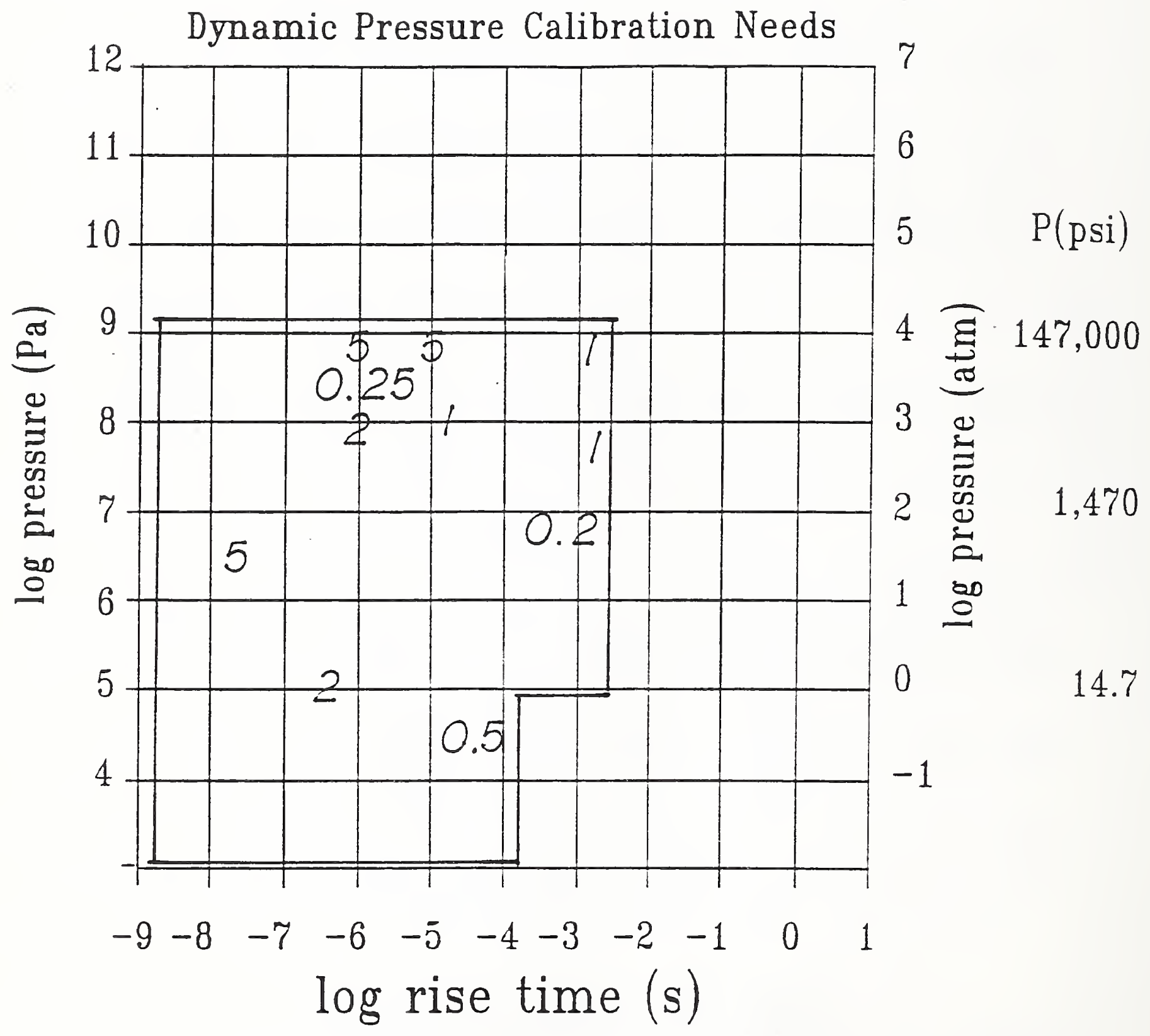

\section{$1 \mathrm{GHz} \quad 1 \mathrm{MHz} \quad 1 \mathrm{kHz}$ frequencies}

Figure 2. The outlined areas in pressure-frequency space represent the transient pressure calibration needs of organizations with representatives attending the workshop other than NASA. The numbers in the areas represent the needed accuracies expressed in percent. 


\section{PREFACE}

Twelve technical talks were presented during the workshop. Speakers were encouraged, but not required, to publish in these proceedings. All twelve talks are here represented in one of three formats: traditional conference papers, abstracts with view graphs, or reprints from archival sources. The last names in parentheses in this summary identify the speaker's published material herein where more information on the topic can be found. 


\begin{abstract}
To be presented at the Workshop on the Measurement of Transient Pressure and Temperature at the National Institute of Standards and Technology (NIST), on the 23-24 Apri 1, 1991.
\end{abstract}

Dynamic High Pressure Measurement

Issues and Instrumentation

Marek L. Winiarz, P. E.

The world of dynamic, transient pressure measurements at high regimes is full of assumptions and uncertainties. First section of the paper will address the key issues of metrology including validation, calibration, and sensor inclusion. Attempts at solutions such as calculational modeling, calibration facilities wi 11 be i 11 ustrated.

The second part will review instrumentation (sensors) used to measure high pressure and stress dynamica11y. Gage examples wi 11 include strain-based devices, piezo-effect devices, and others. Mounting issues, inclusion problems and data acquisition wi 11 be addressed for each gage.

The concluding section will provide an overview of the state-of-the-art techniques used to measure pressure in turbine engine design and development at Genera1 Electric Aircraft Engines. It wi11 i 11 ustrate how engineers approach these critical measurements in the presence of temperature and air-flow fluctuations. 


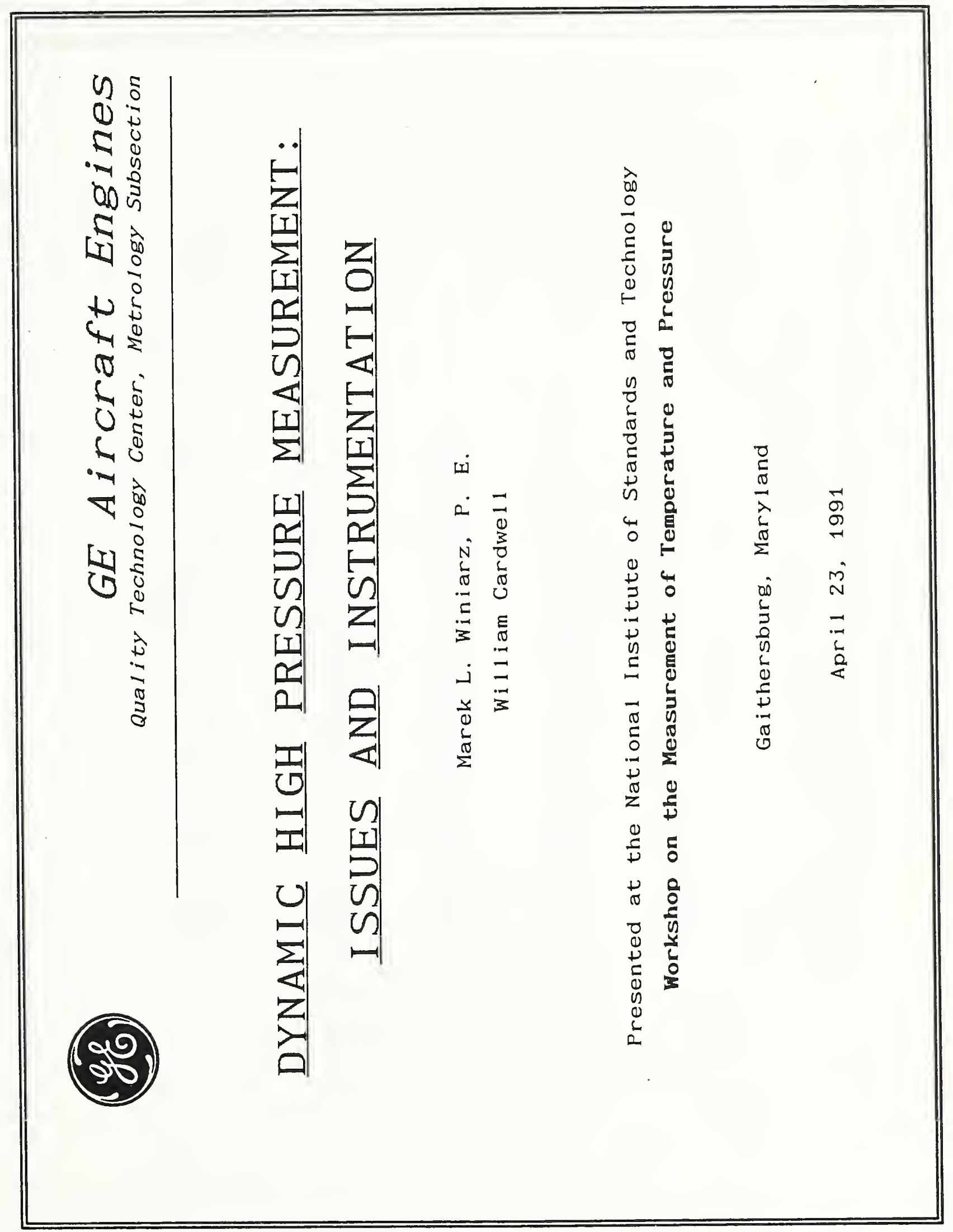




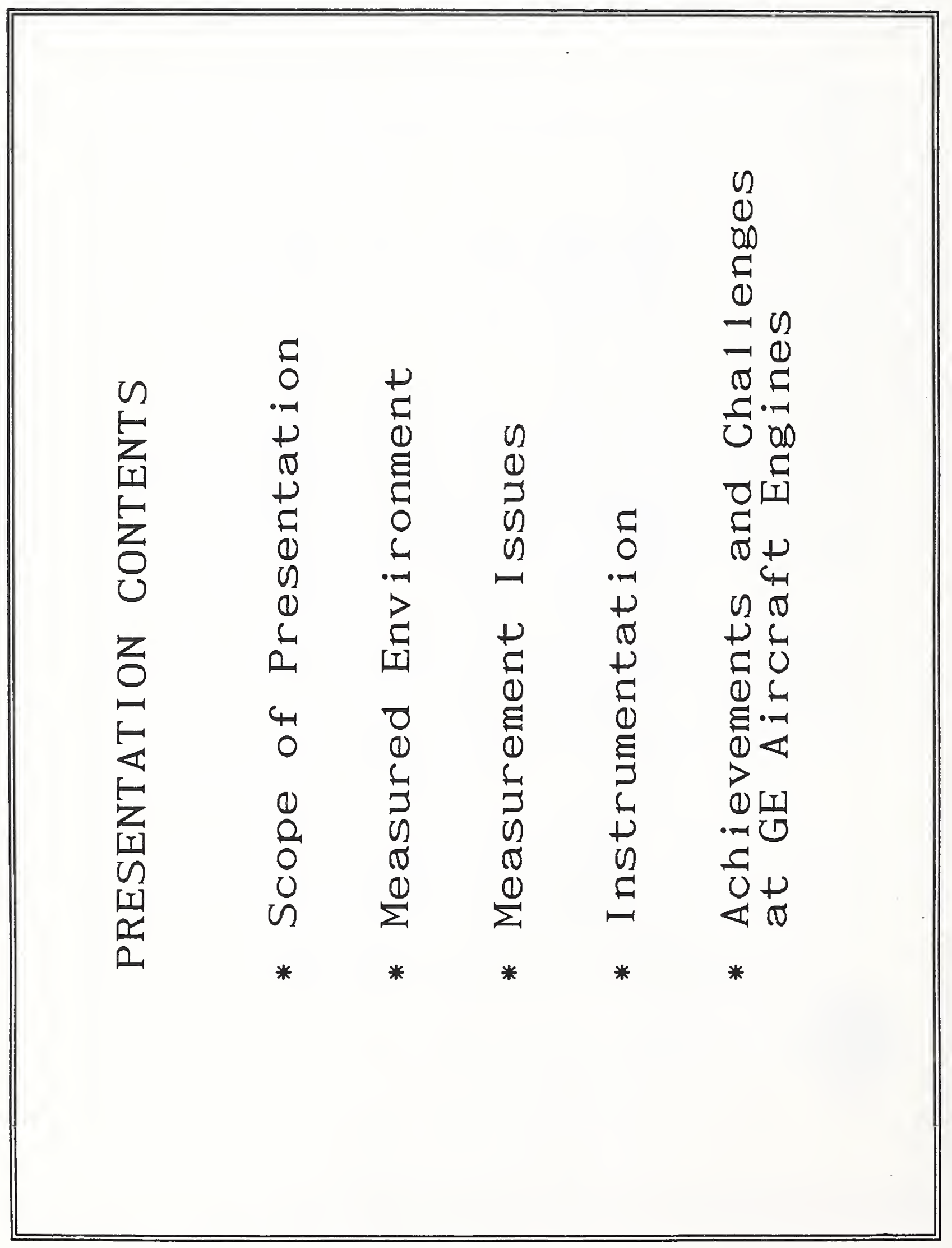




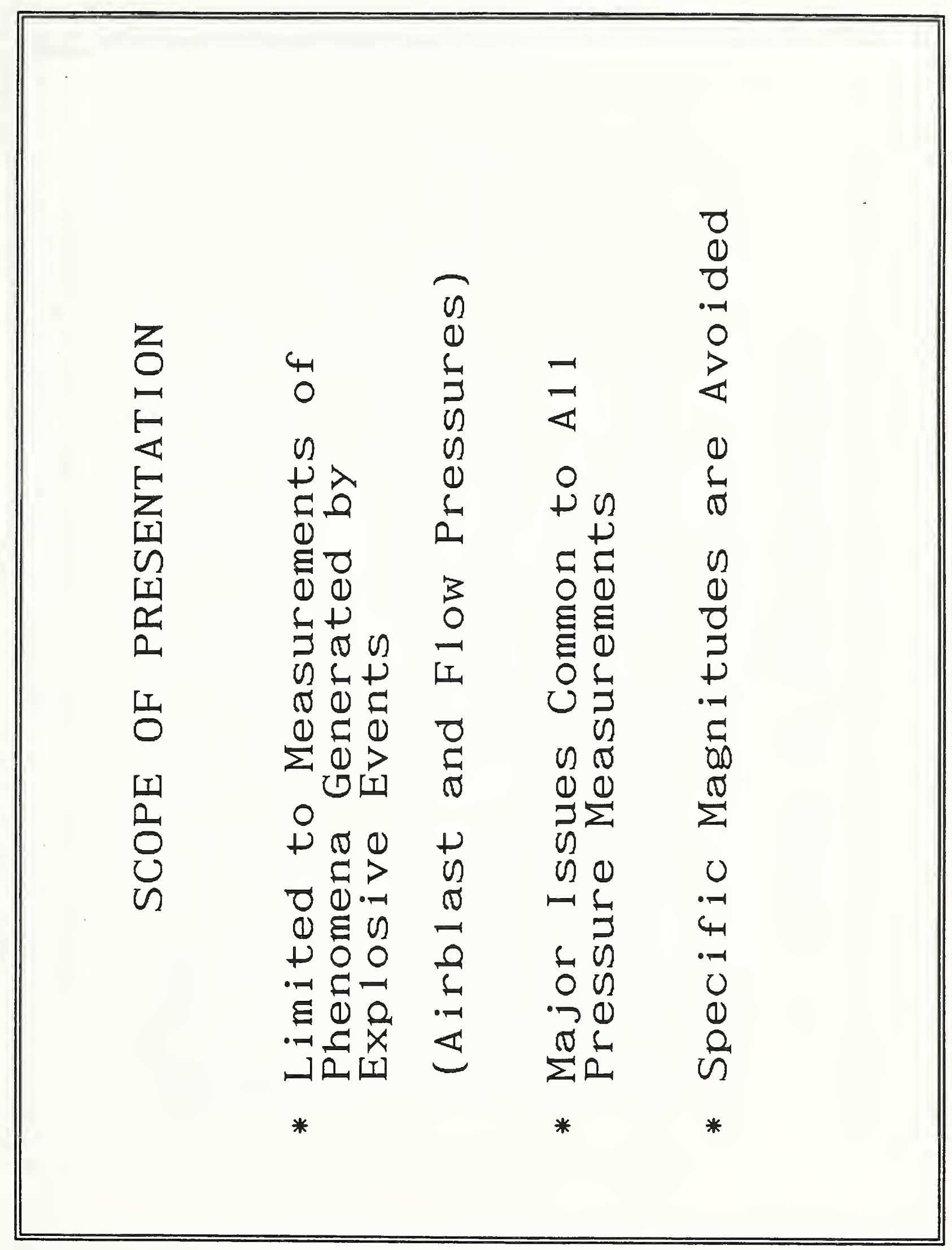




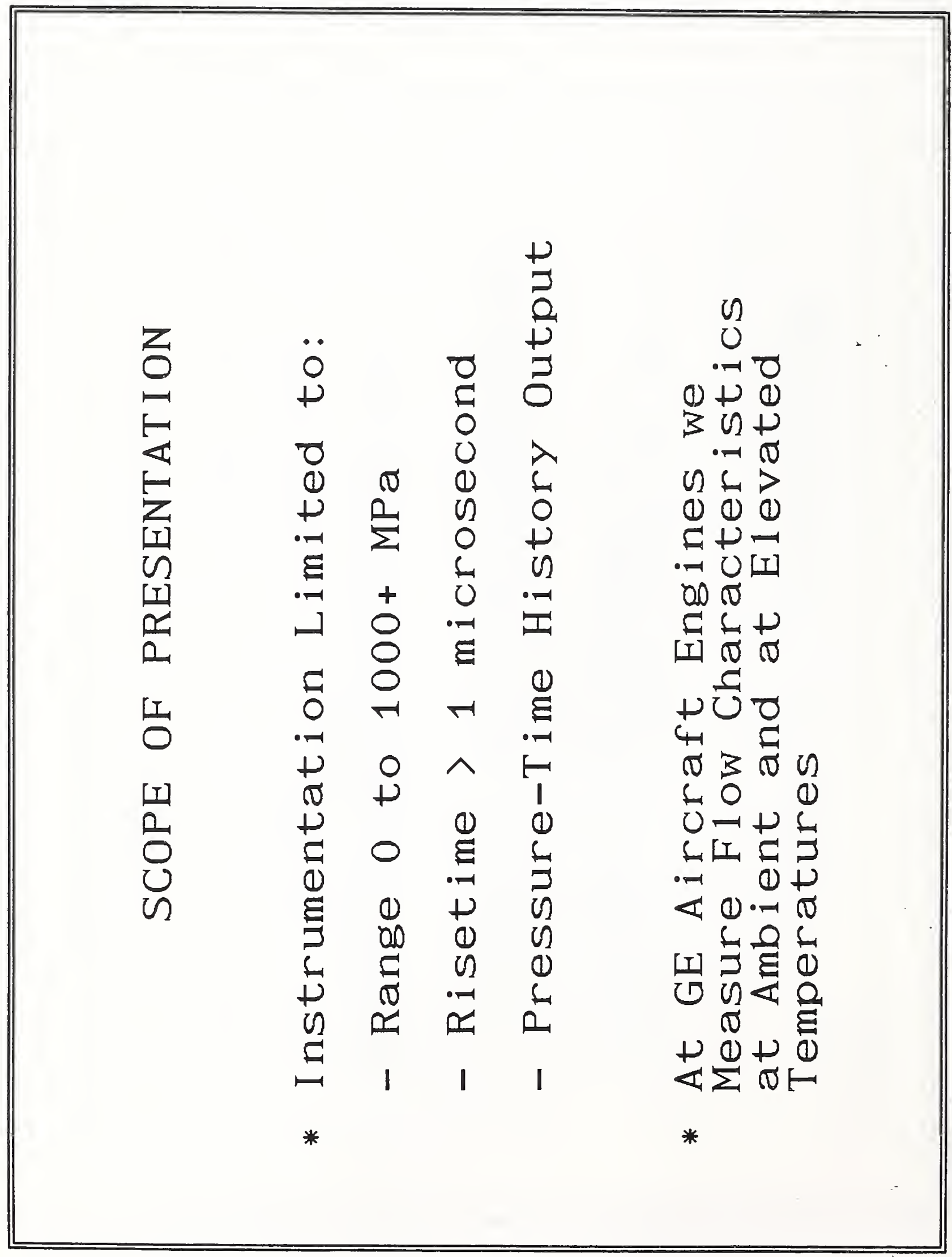




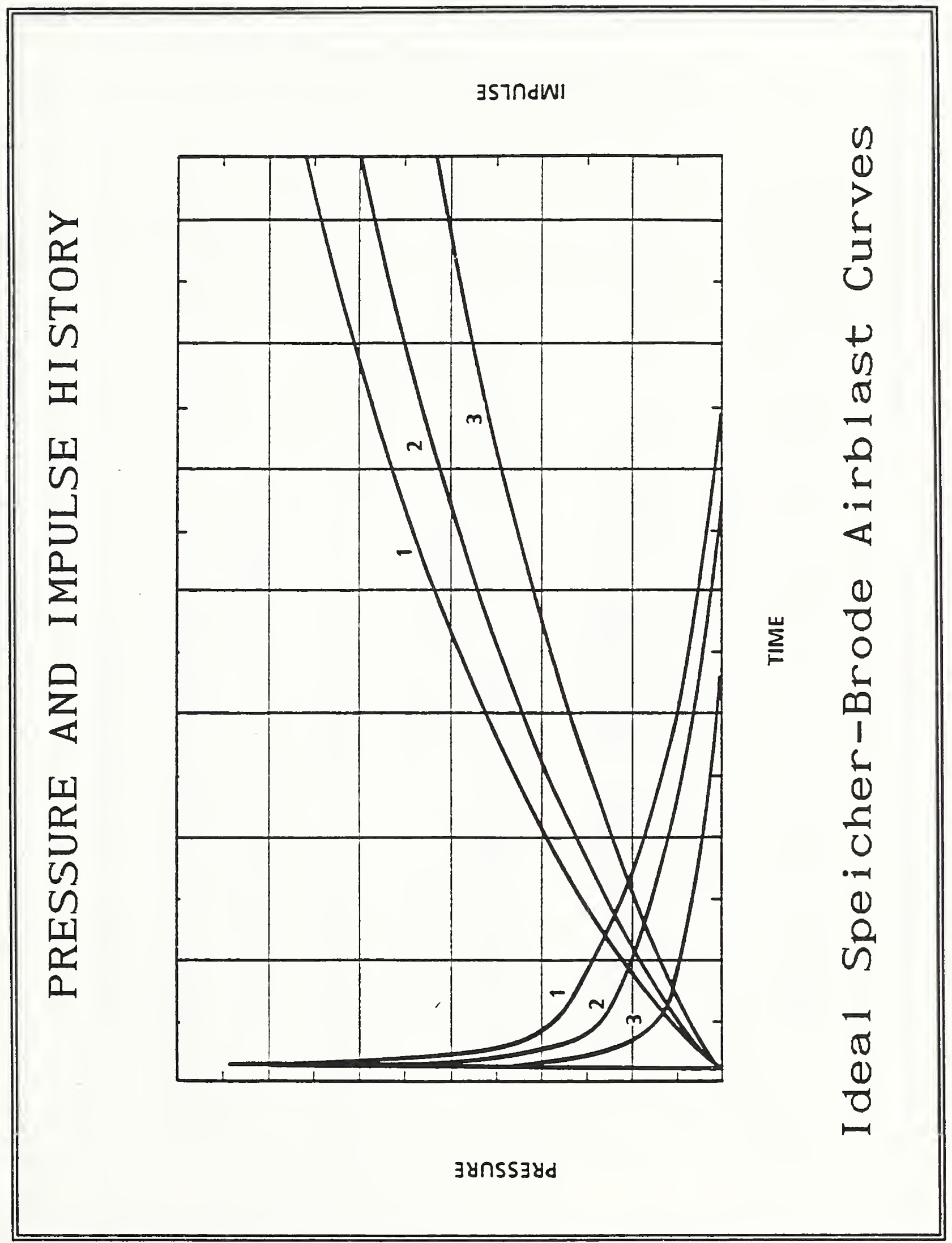




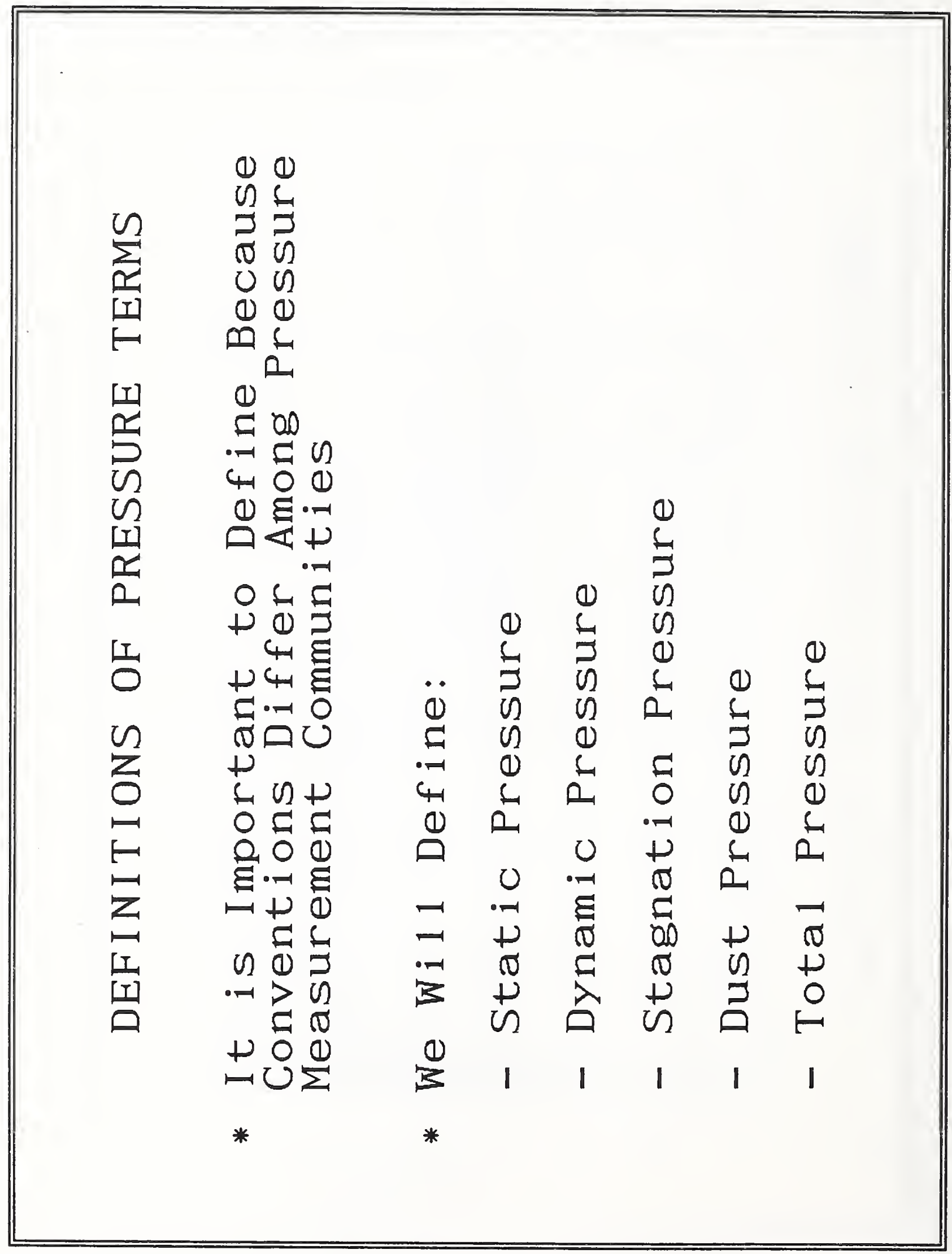




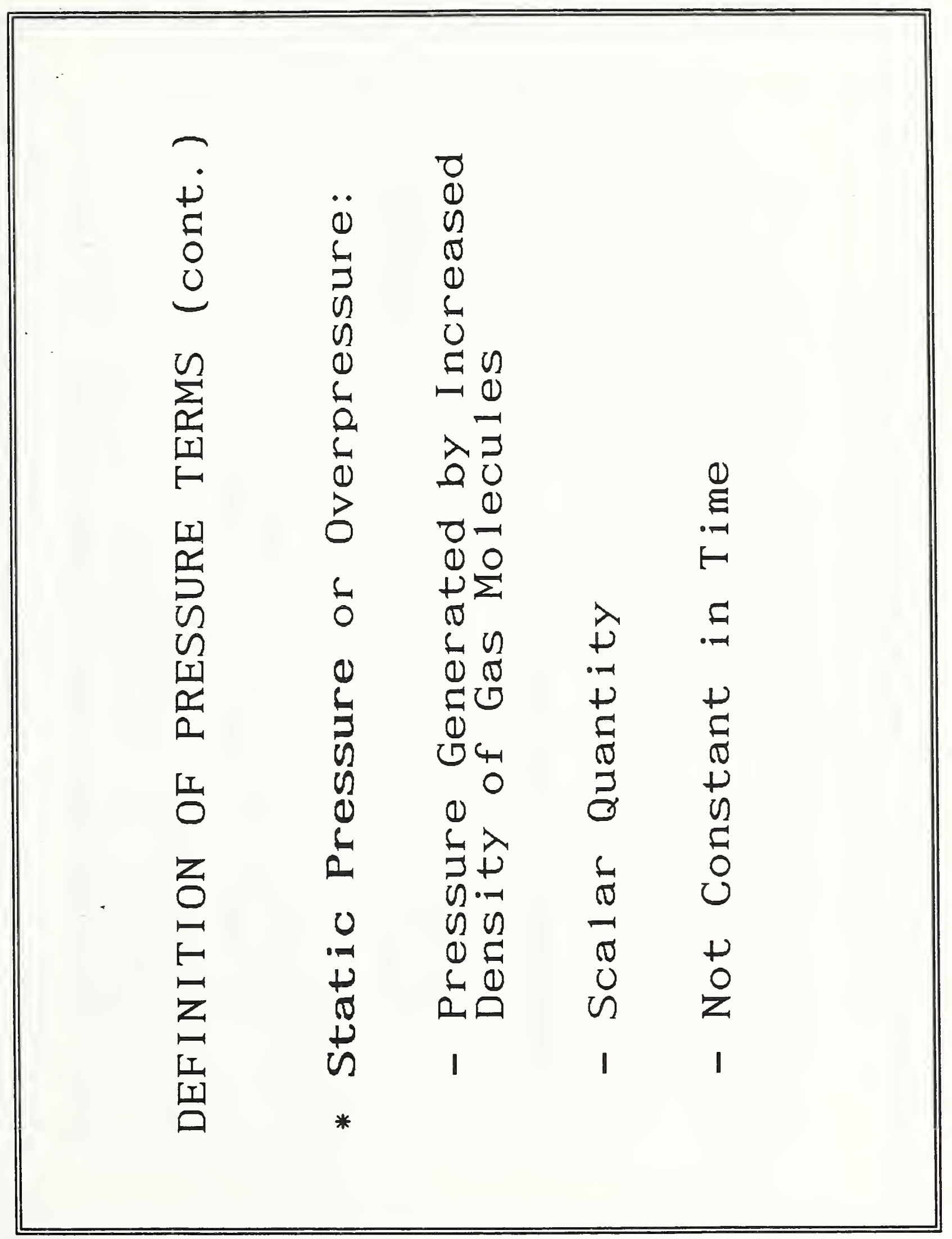




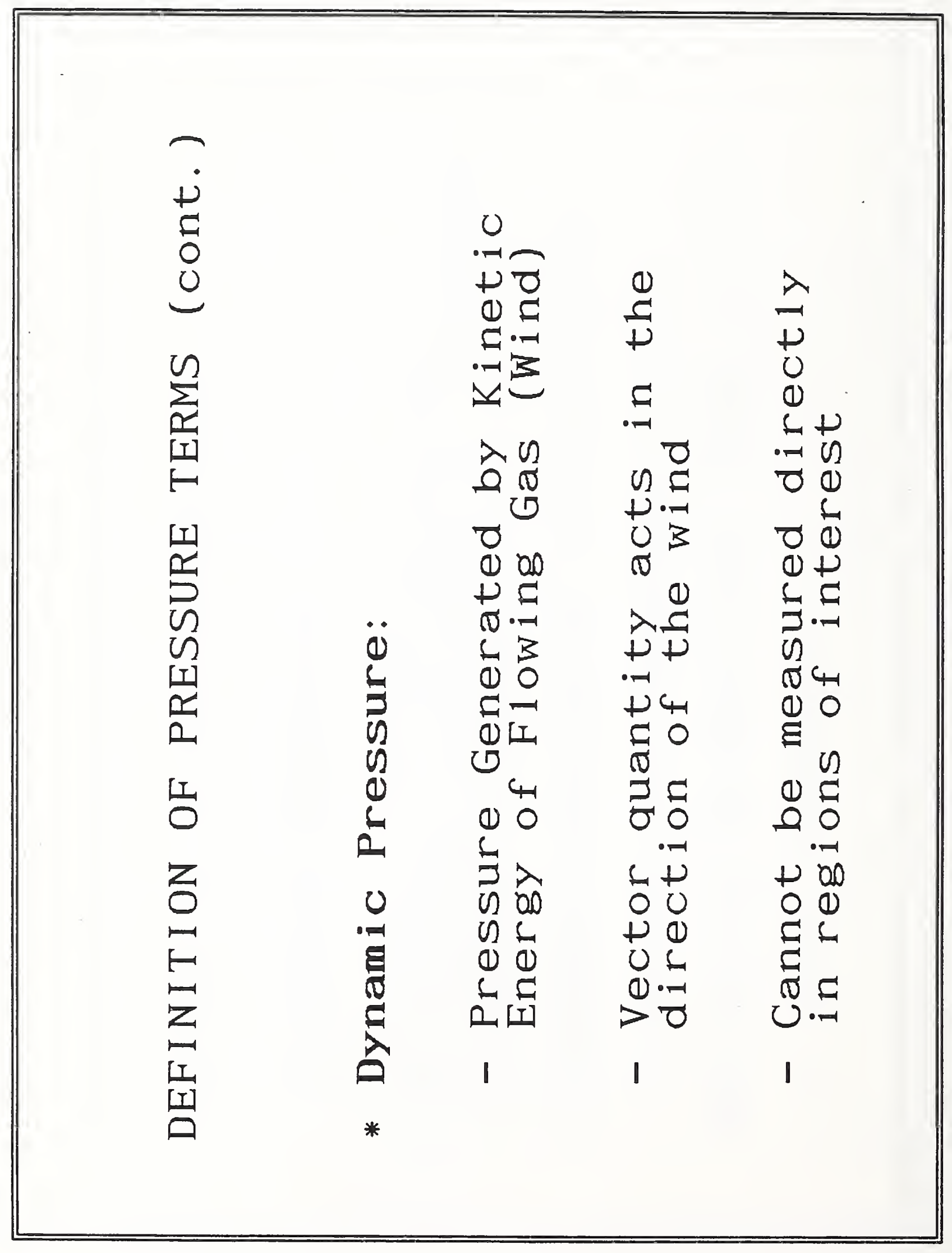




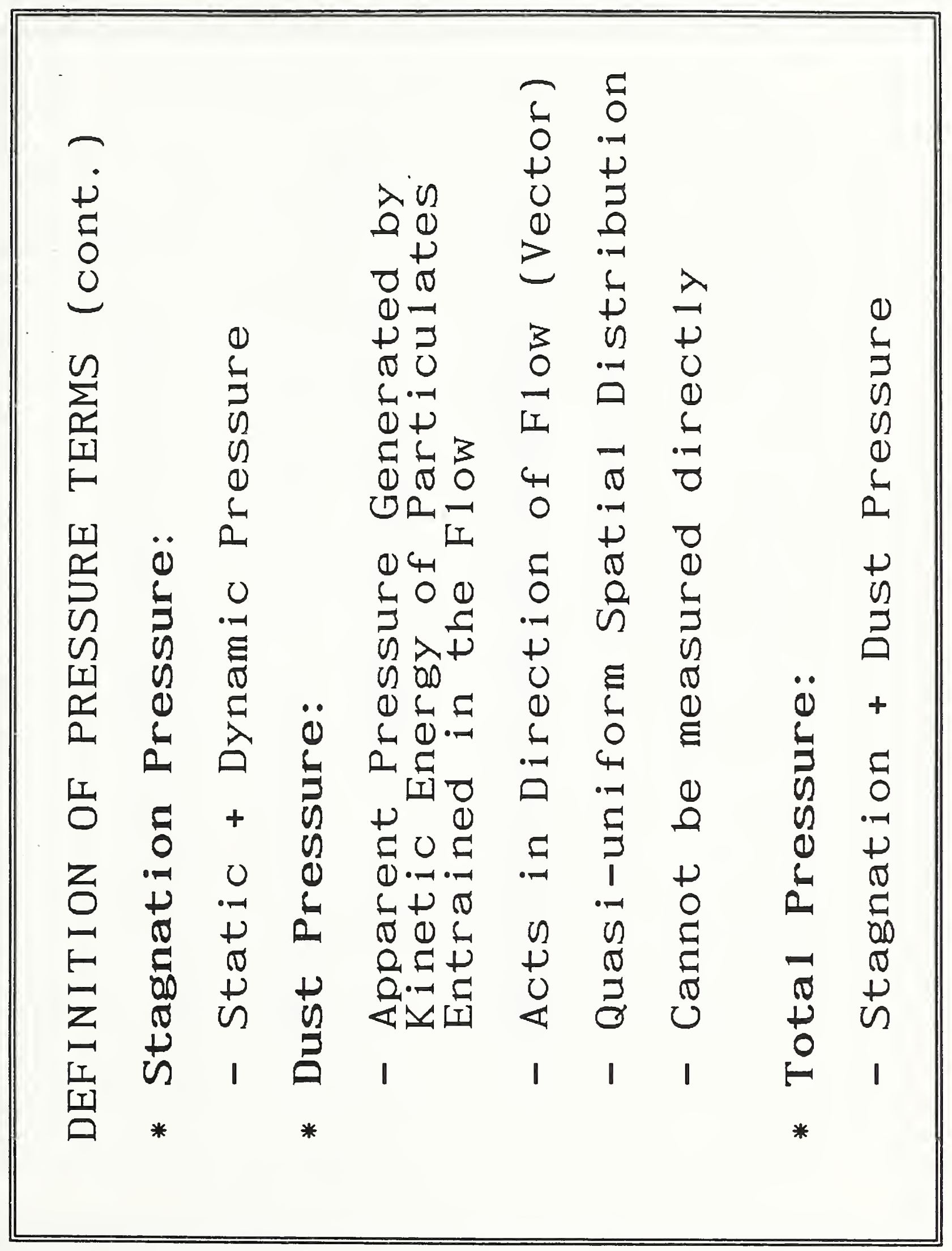




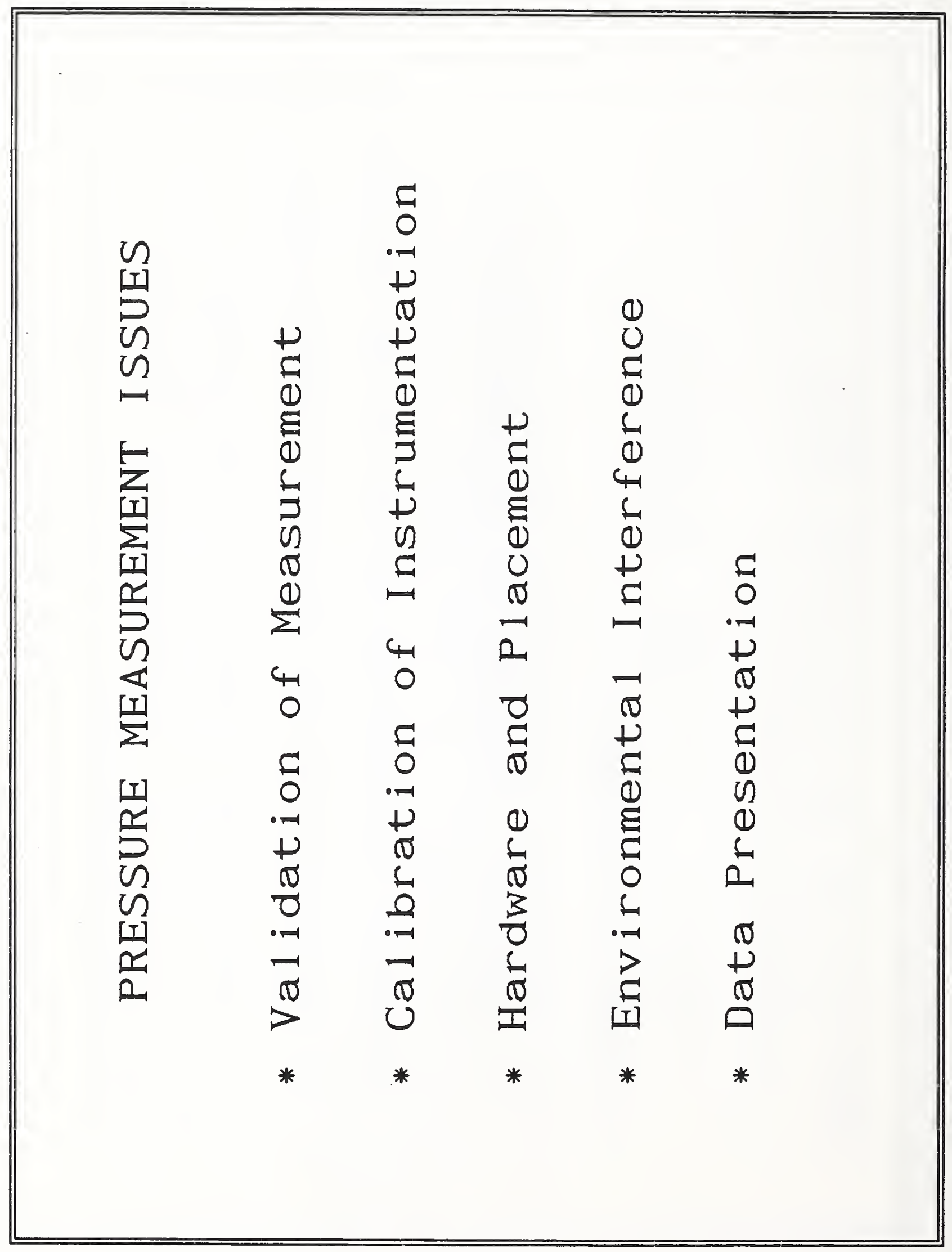




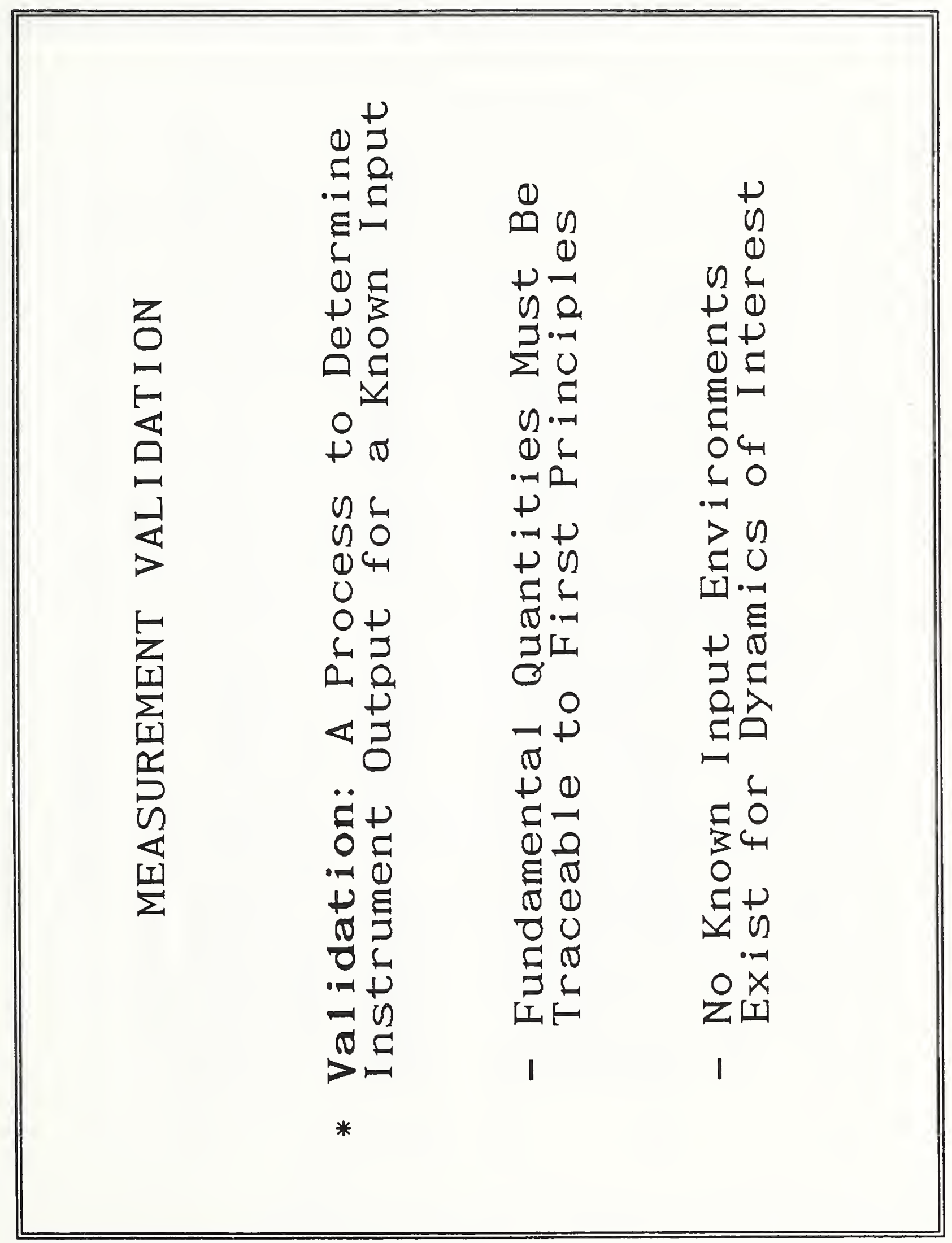




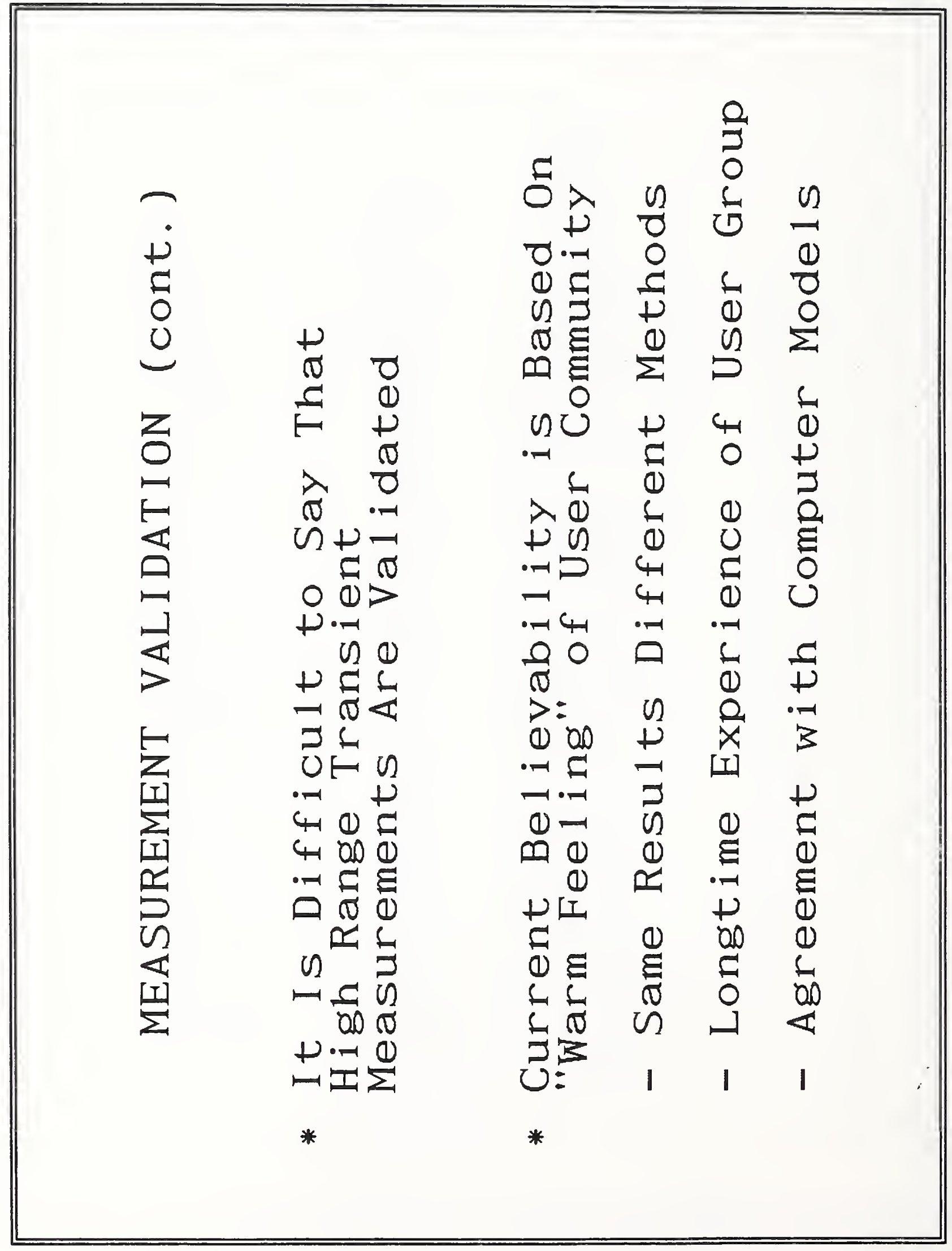




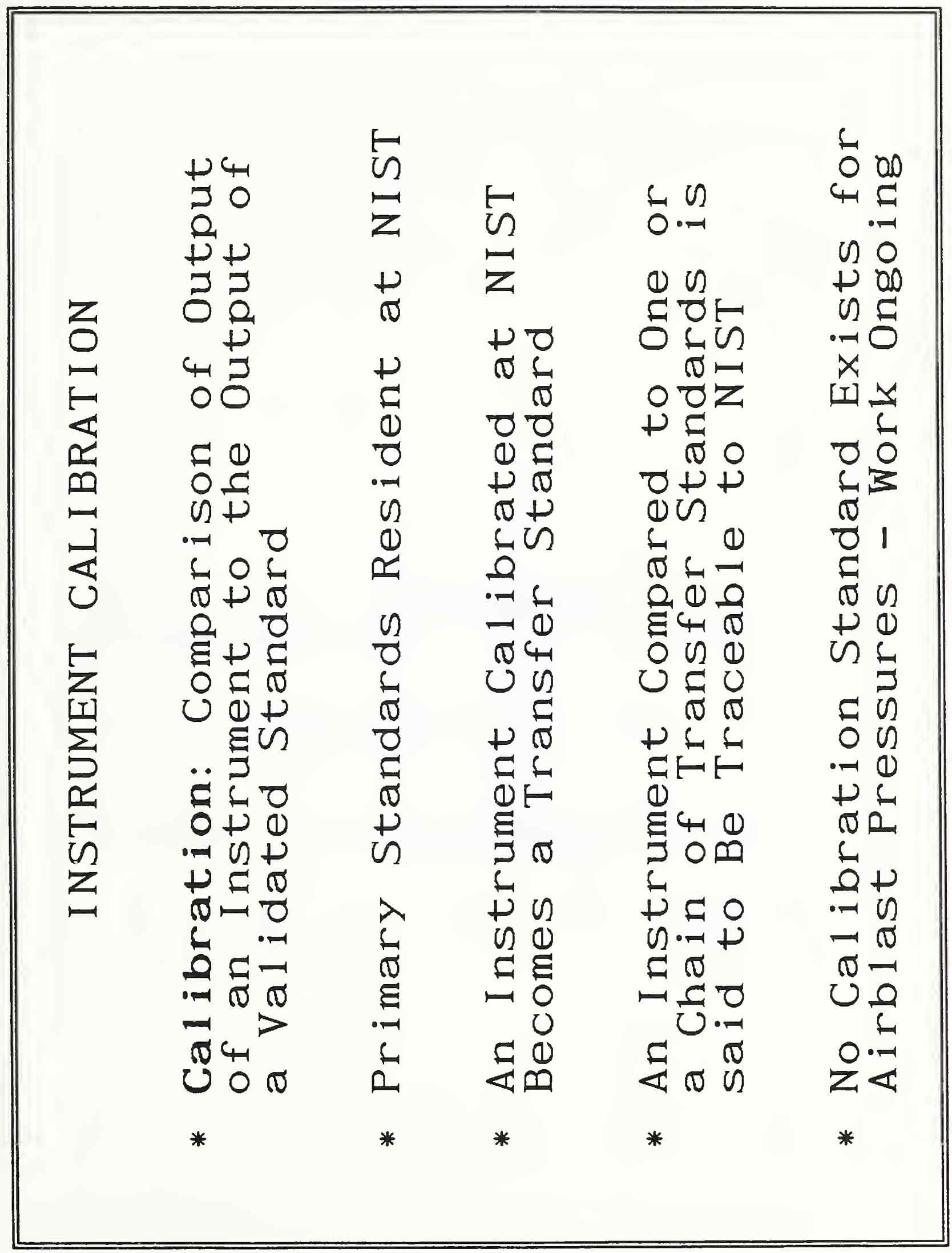




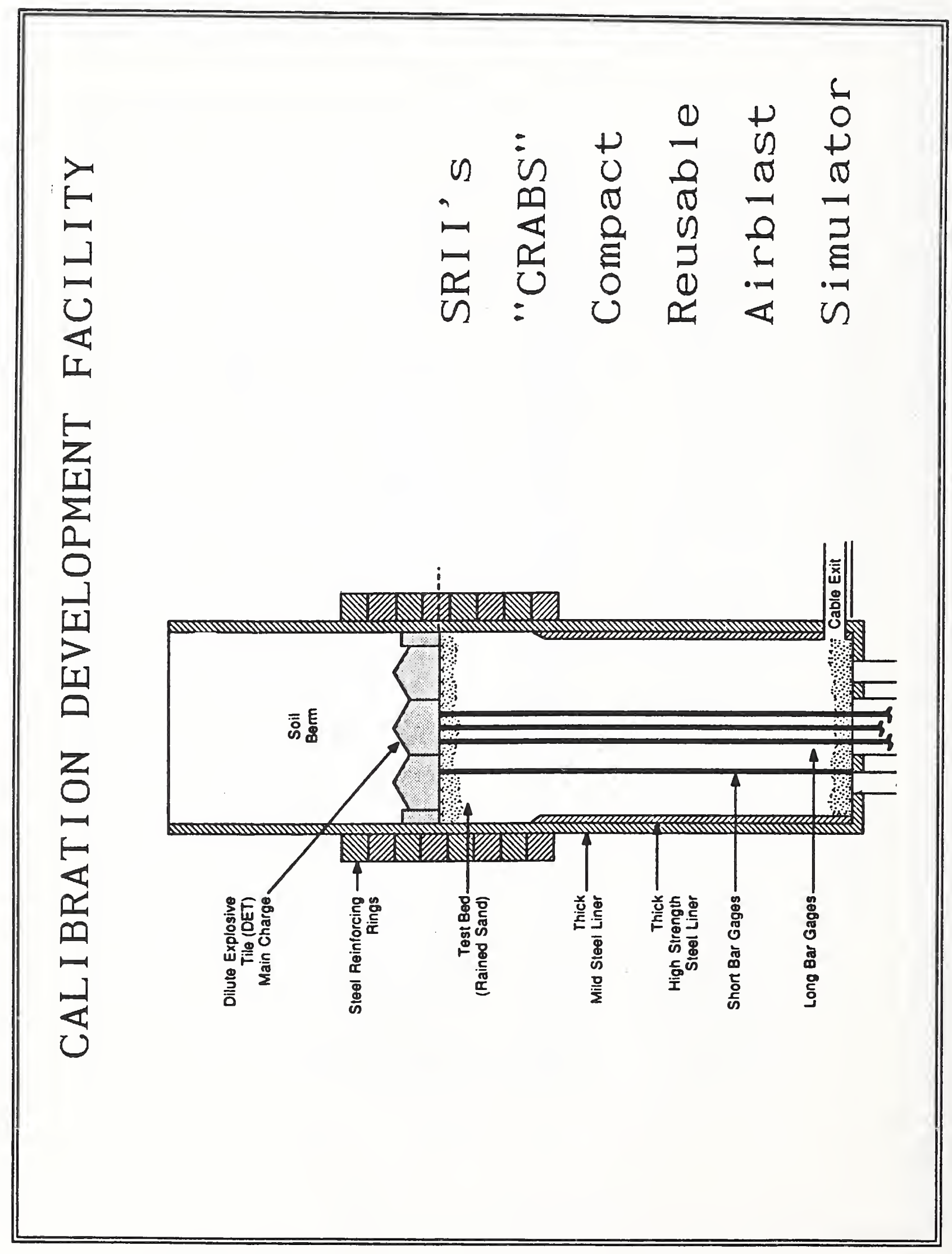




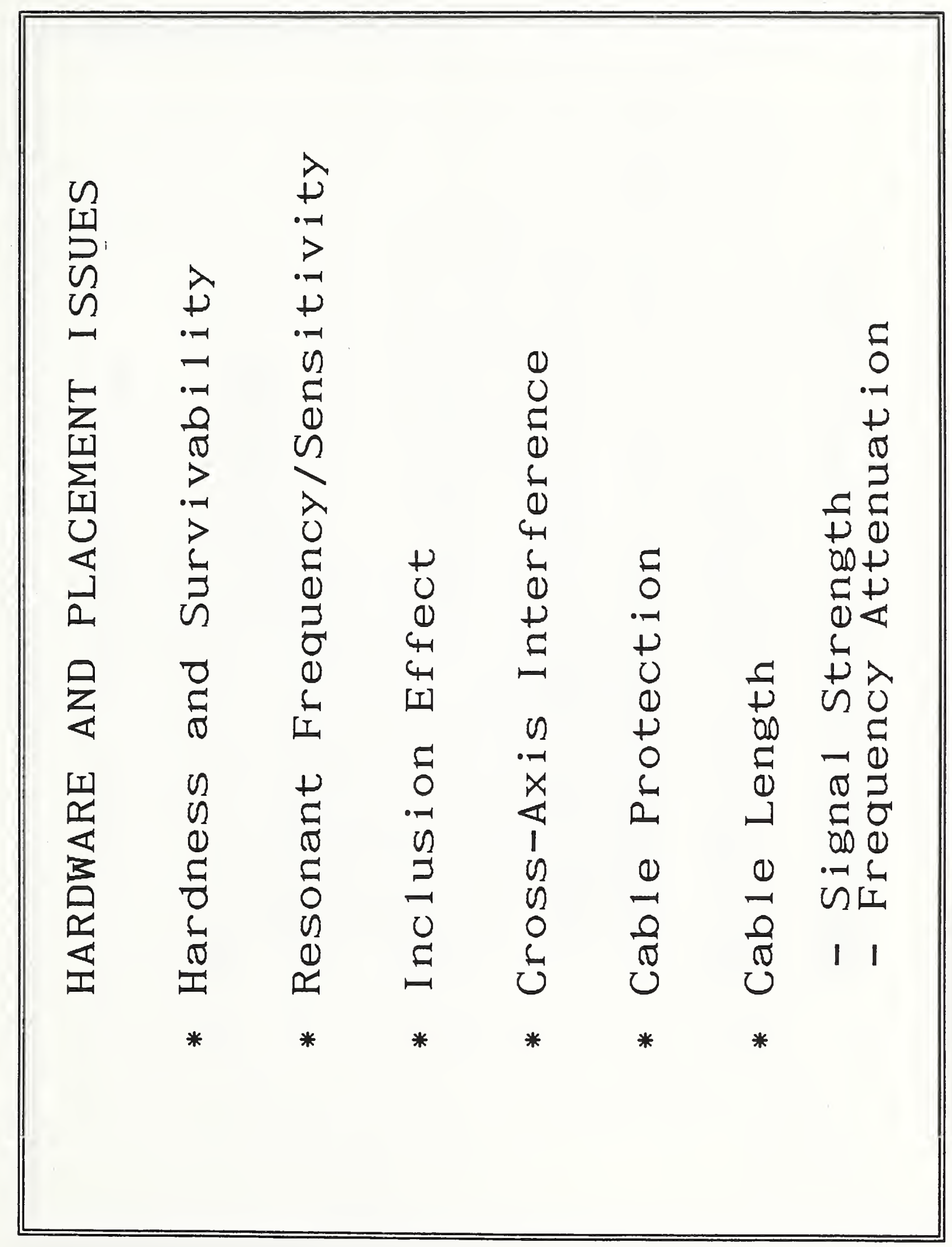




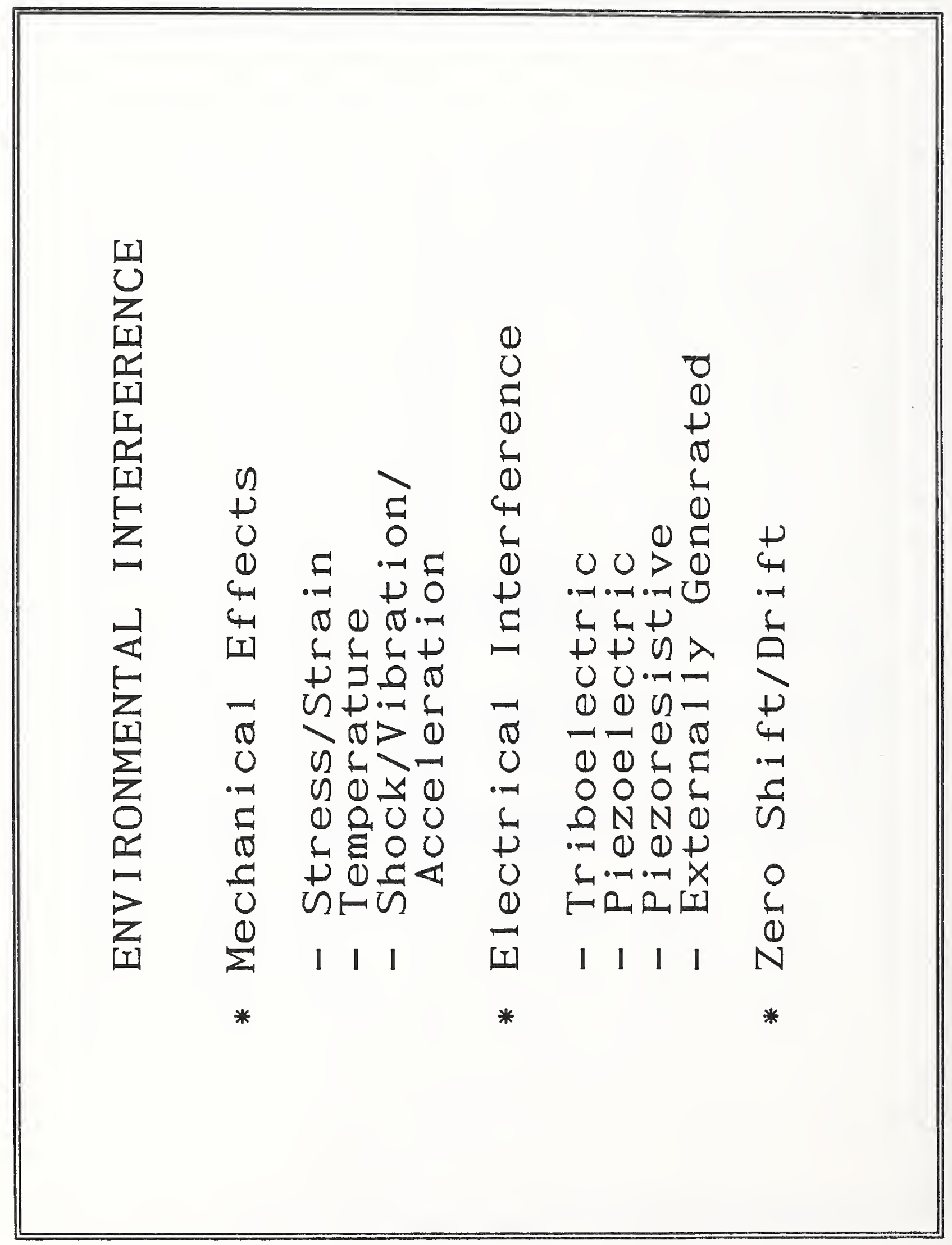




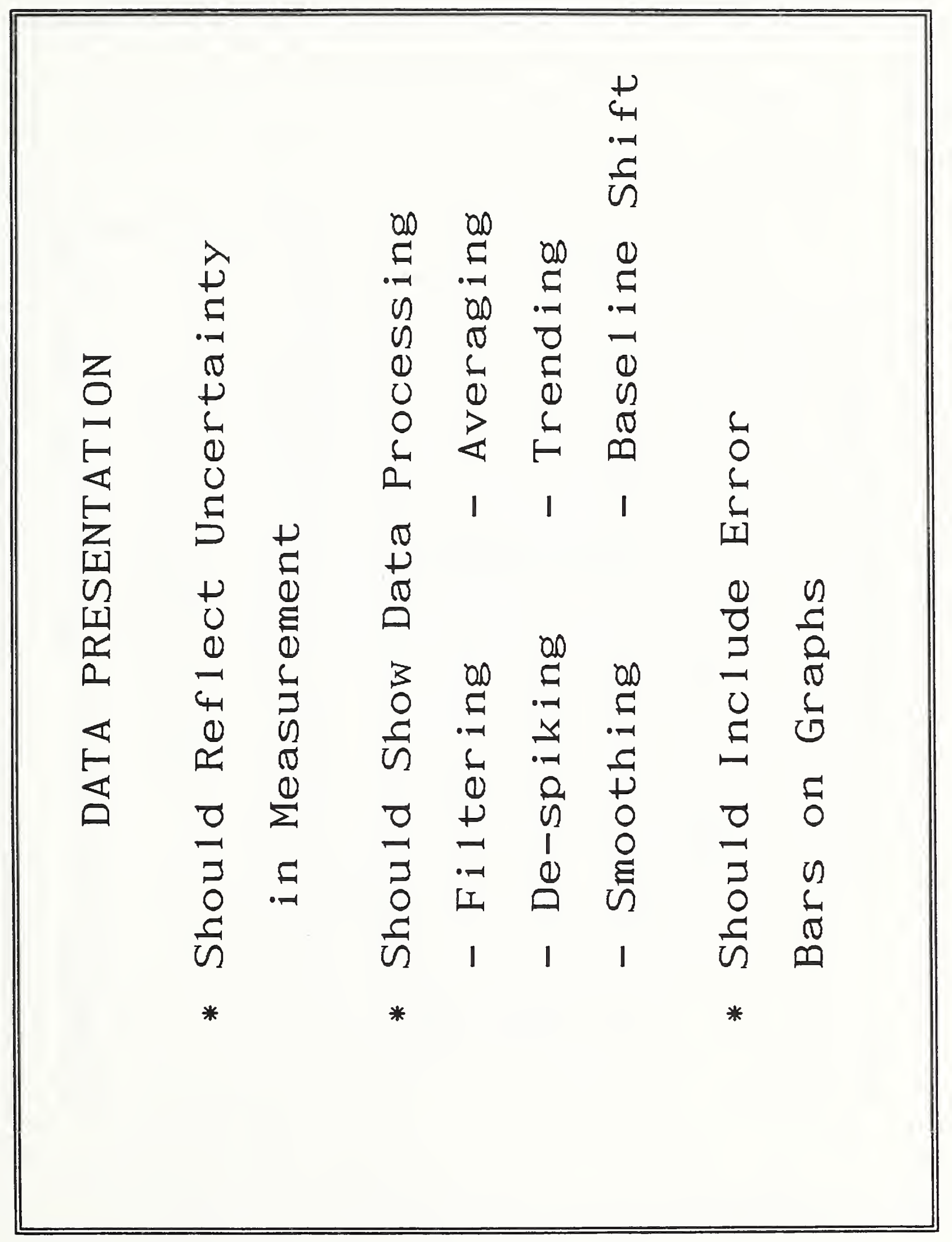




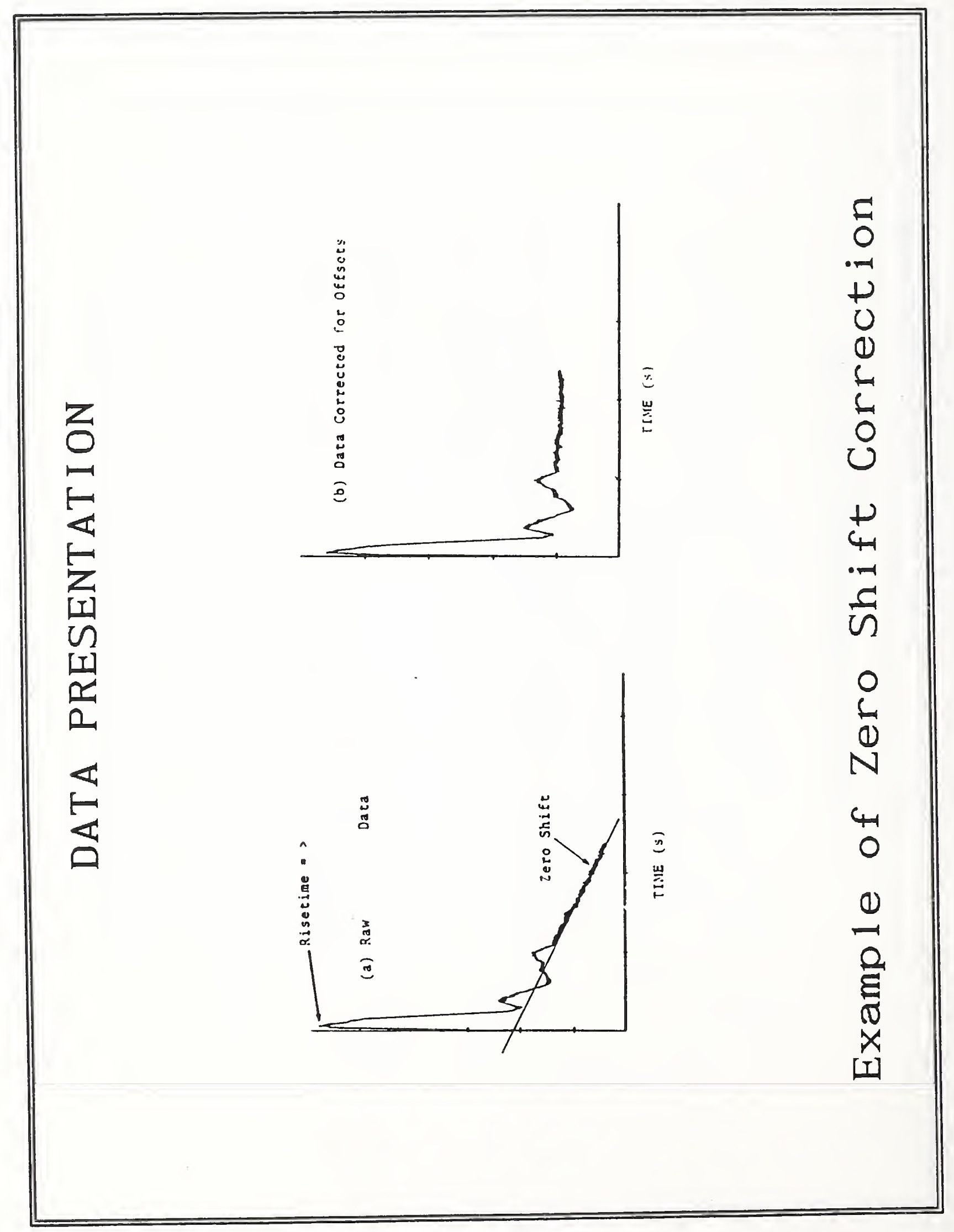




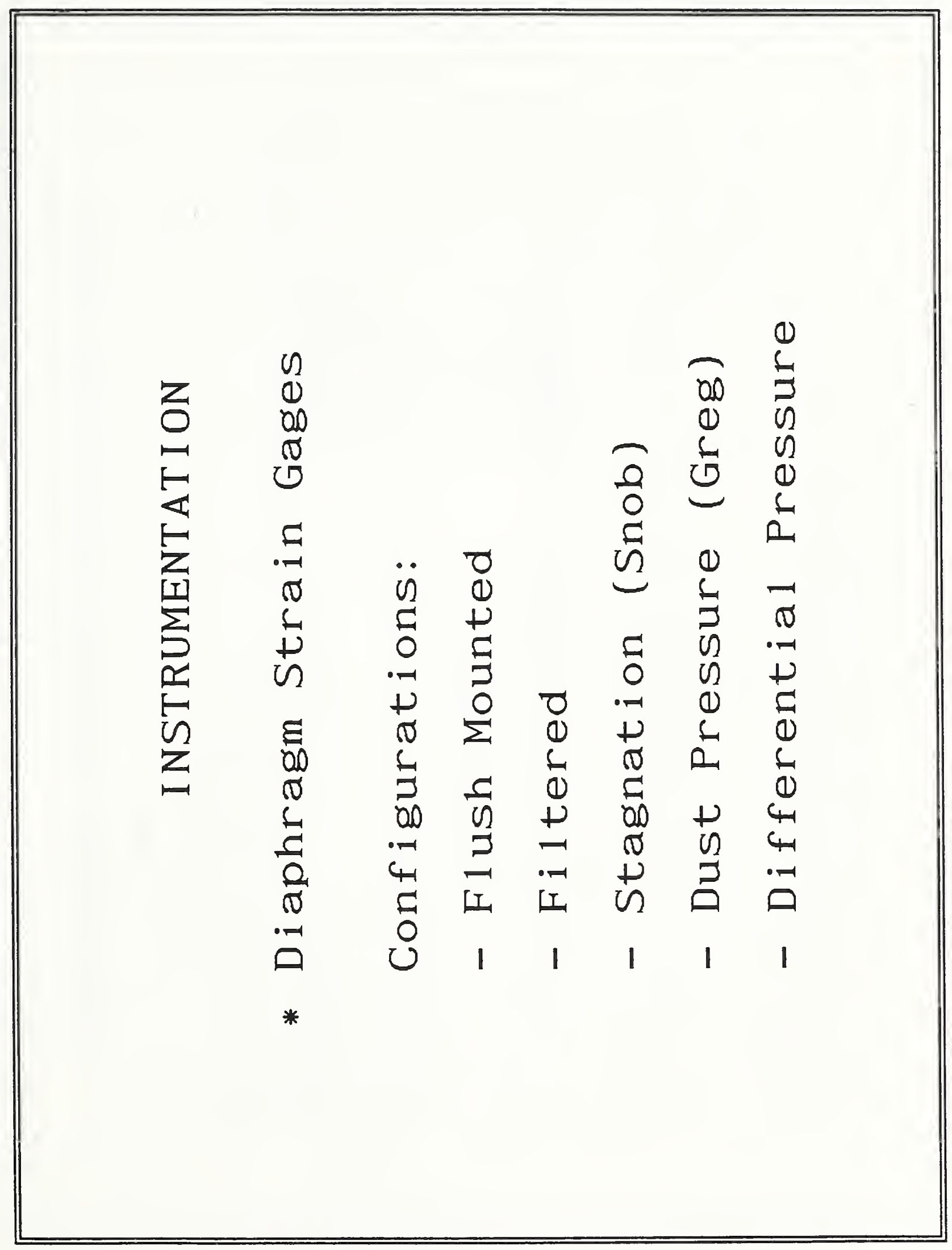




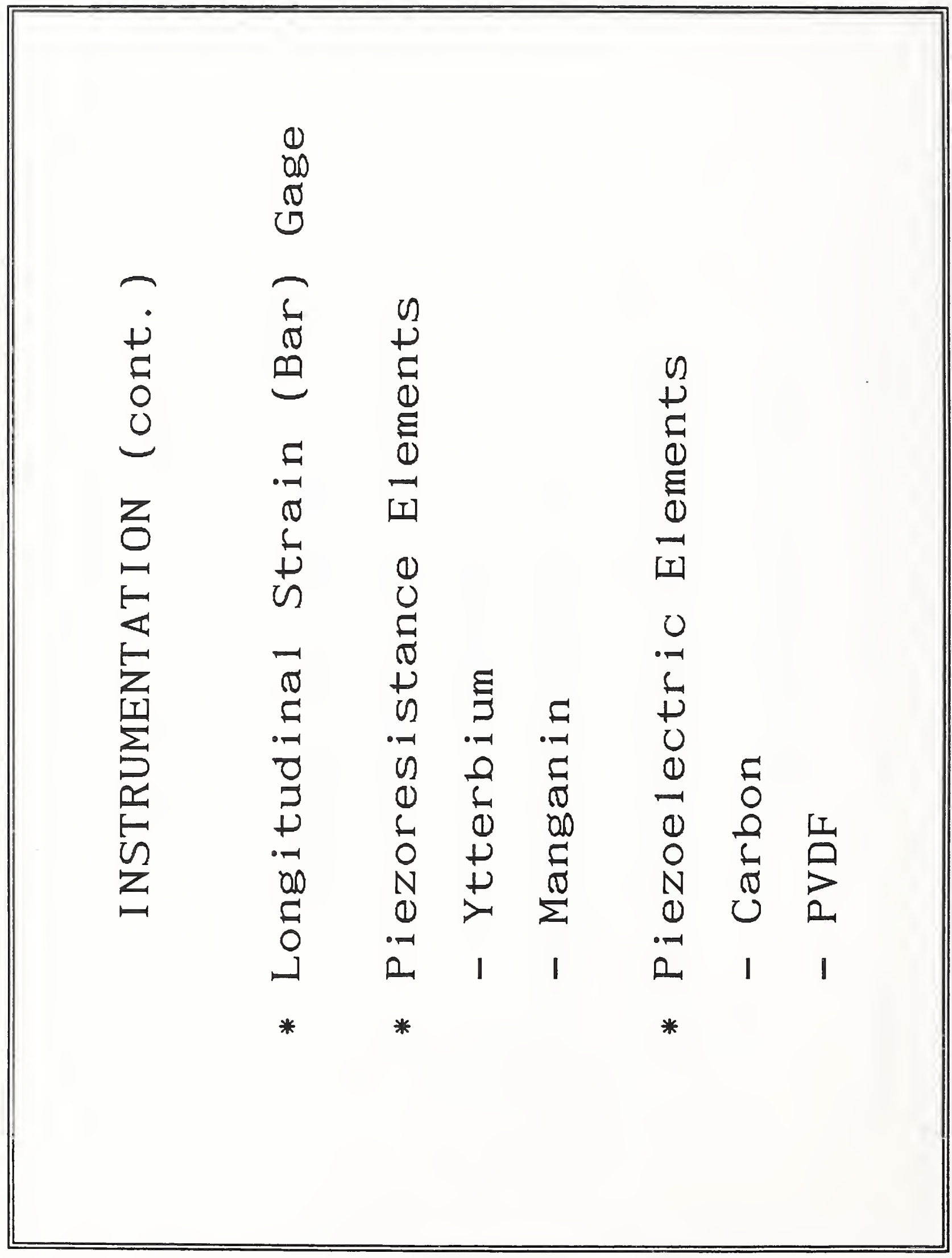




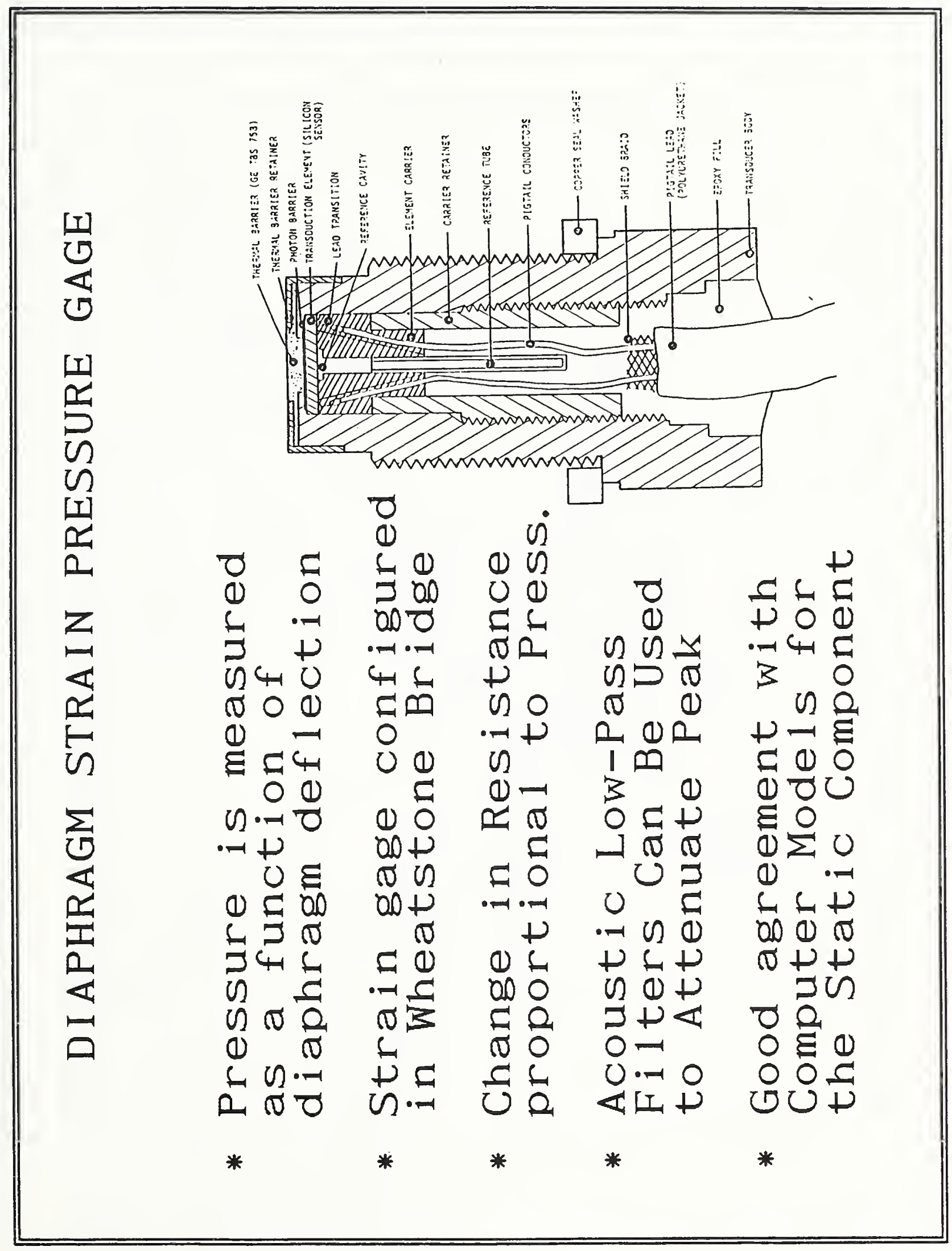




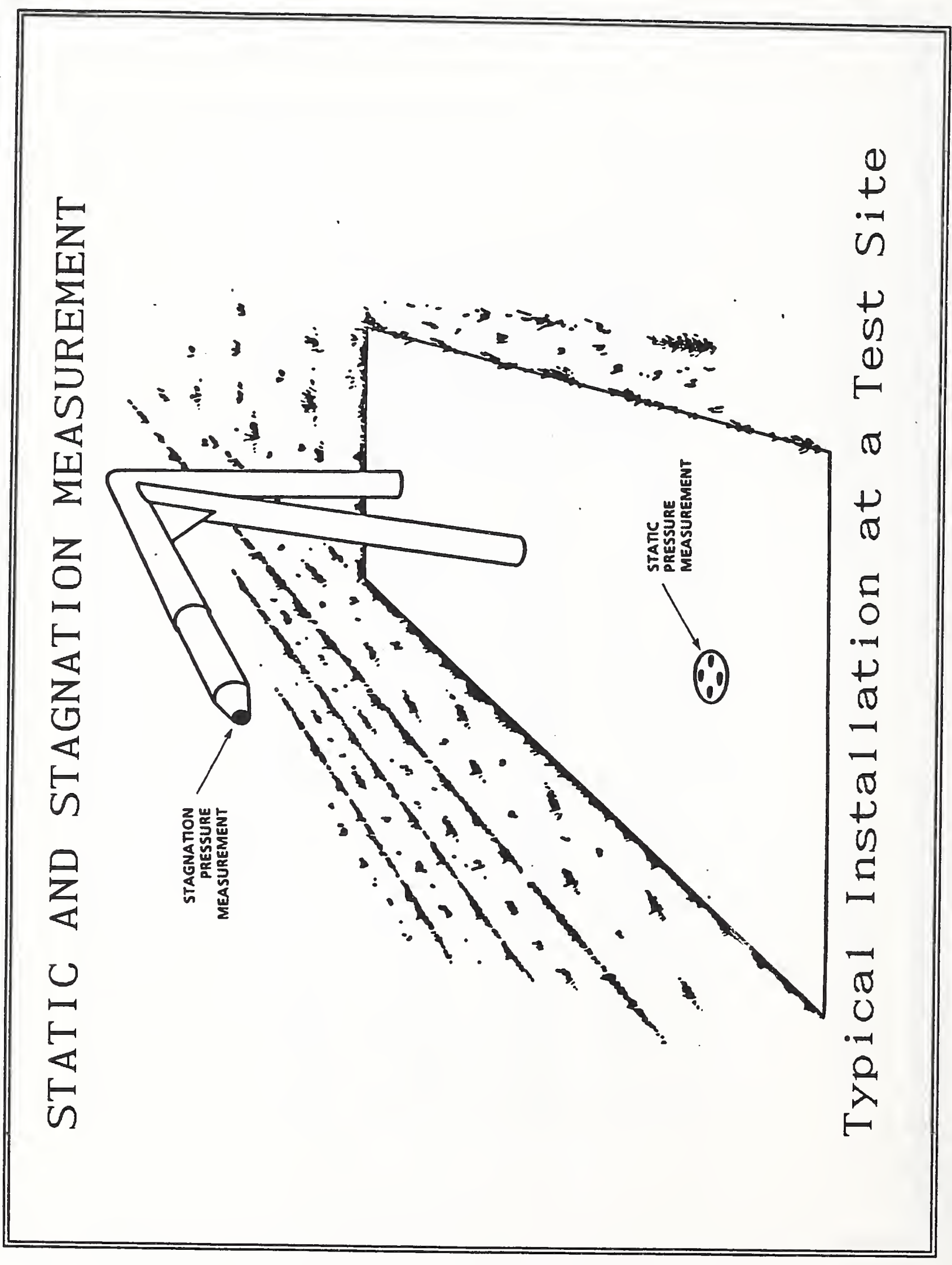




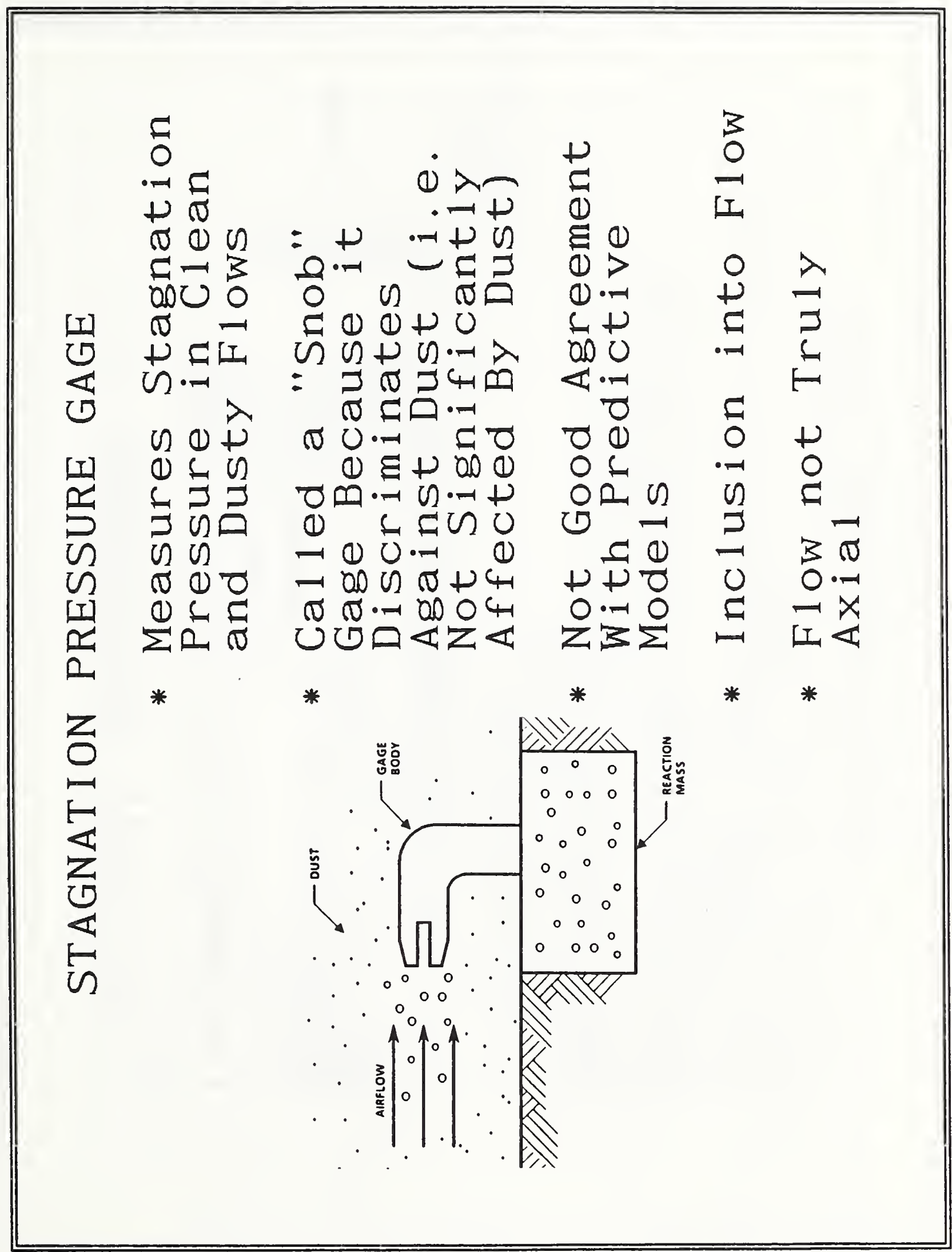




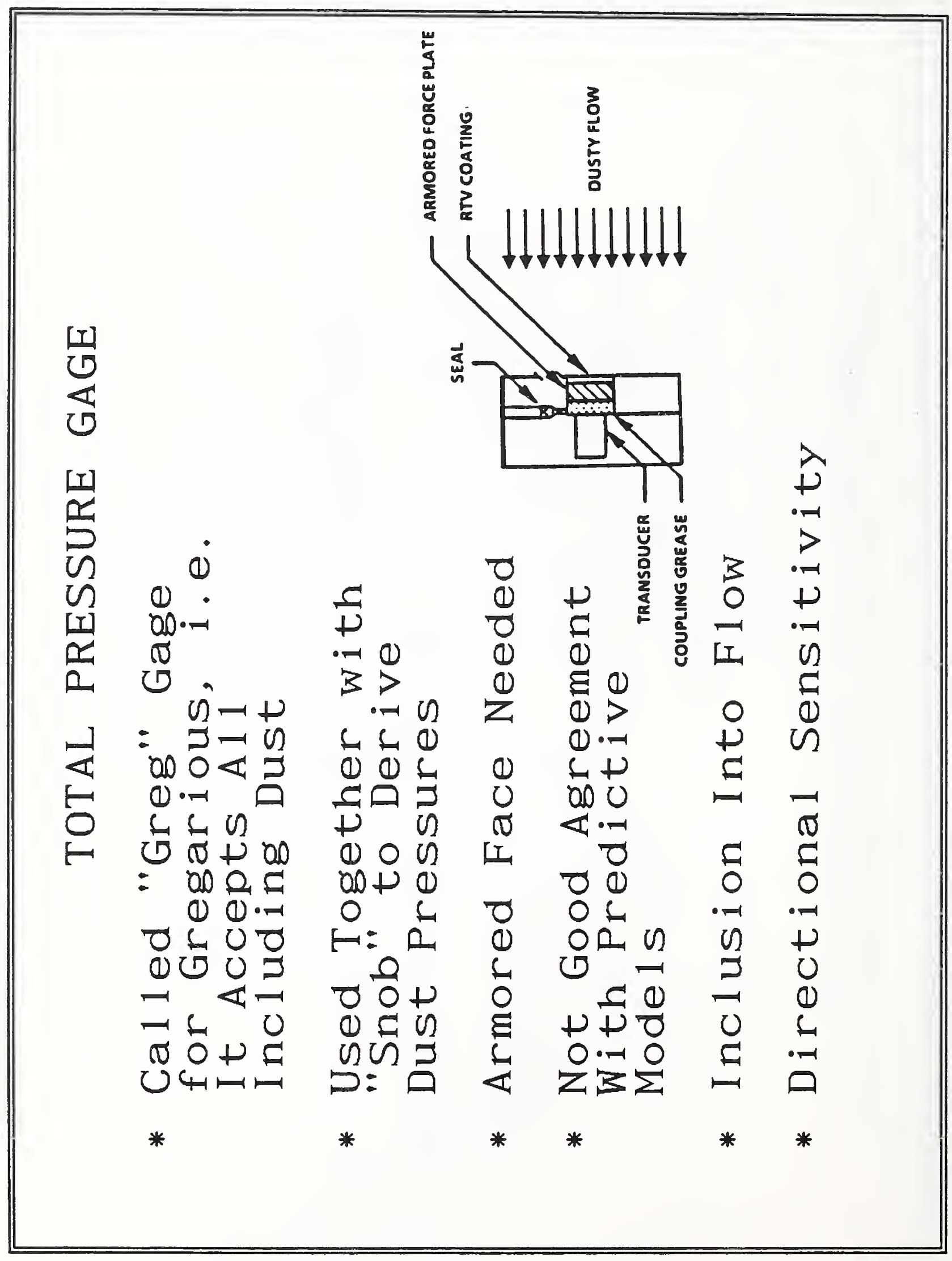




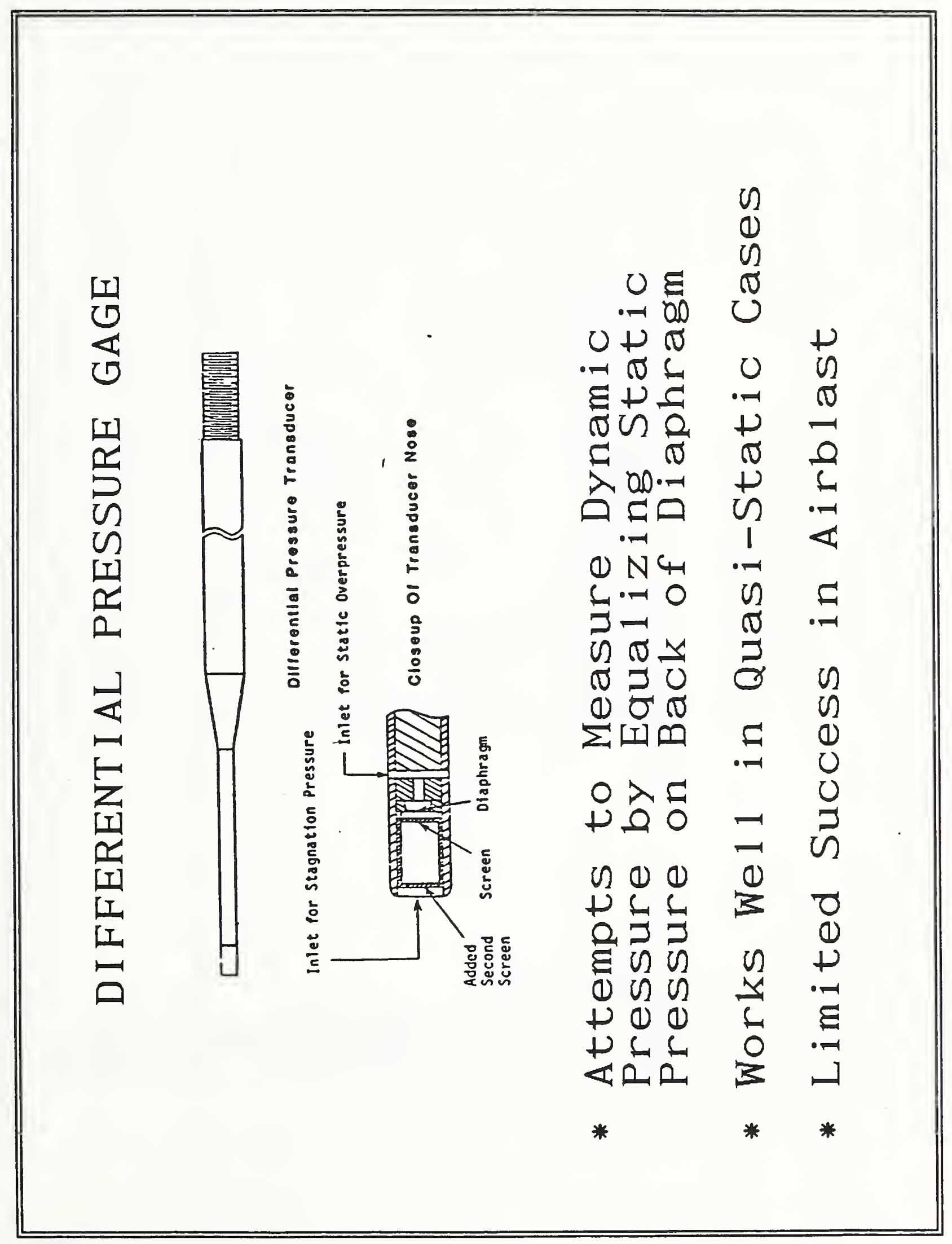




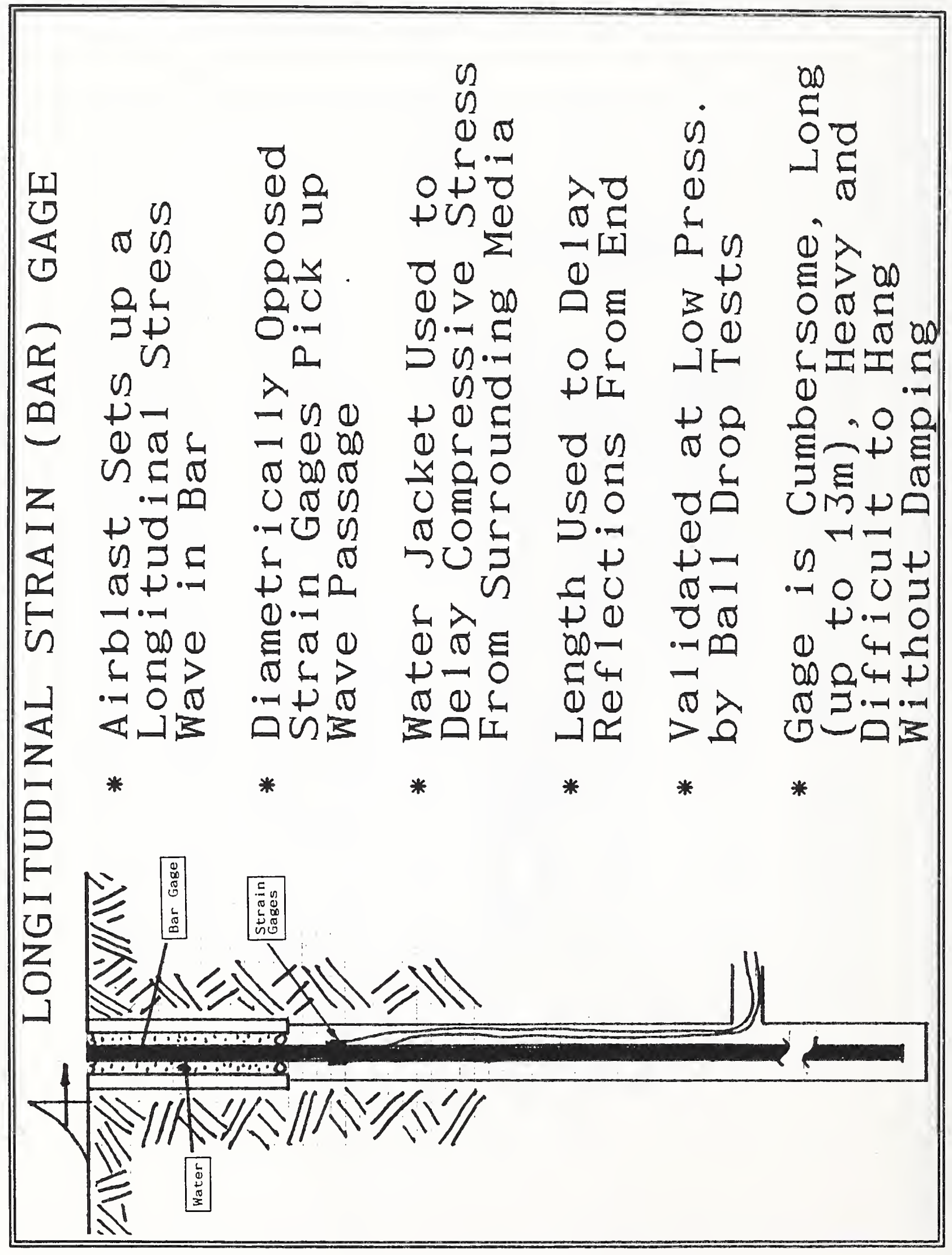




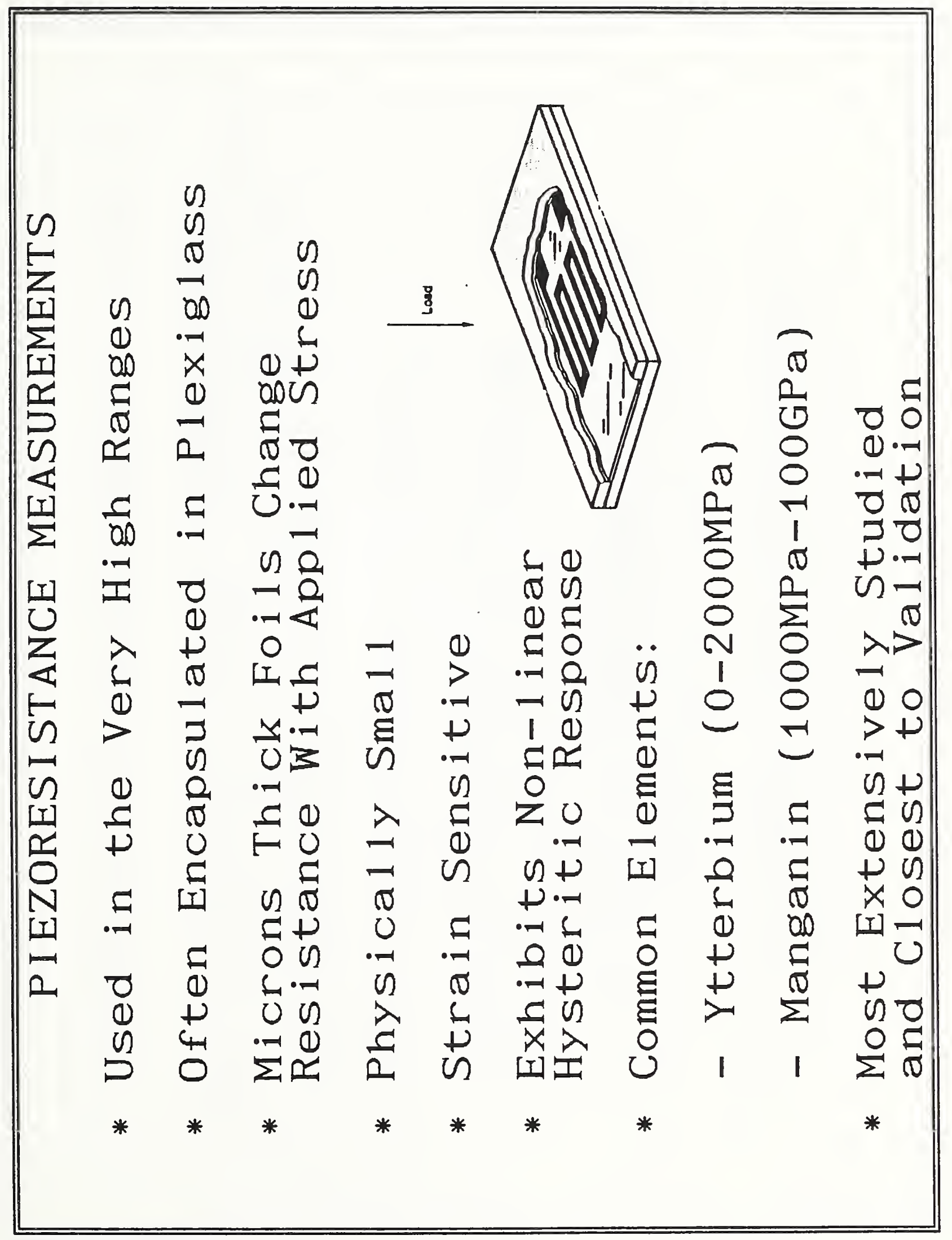




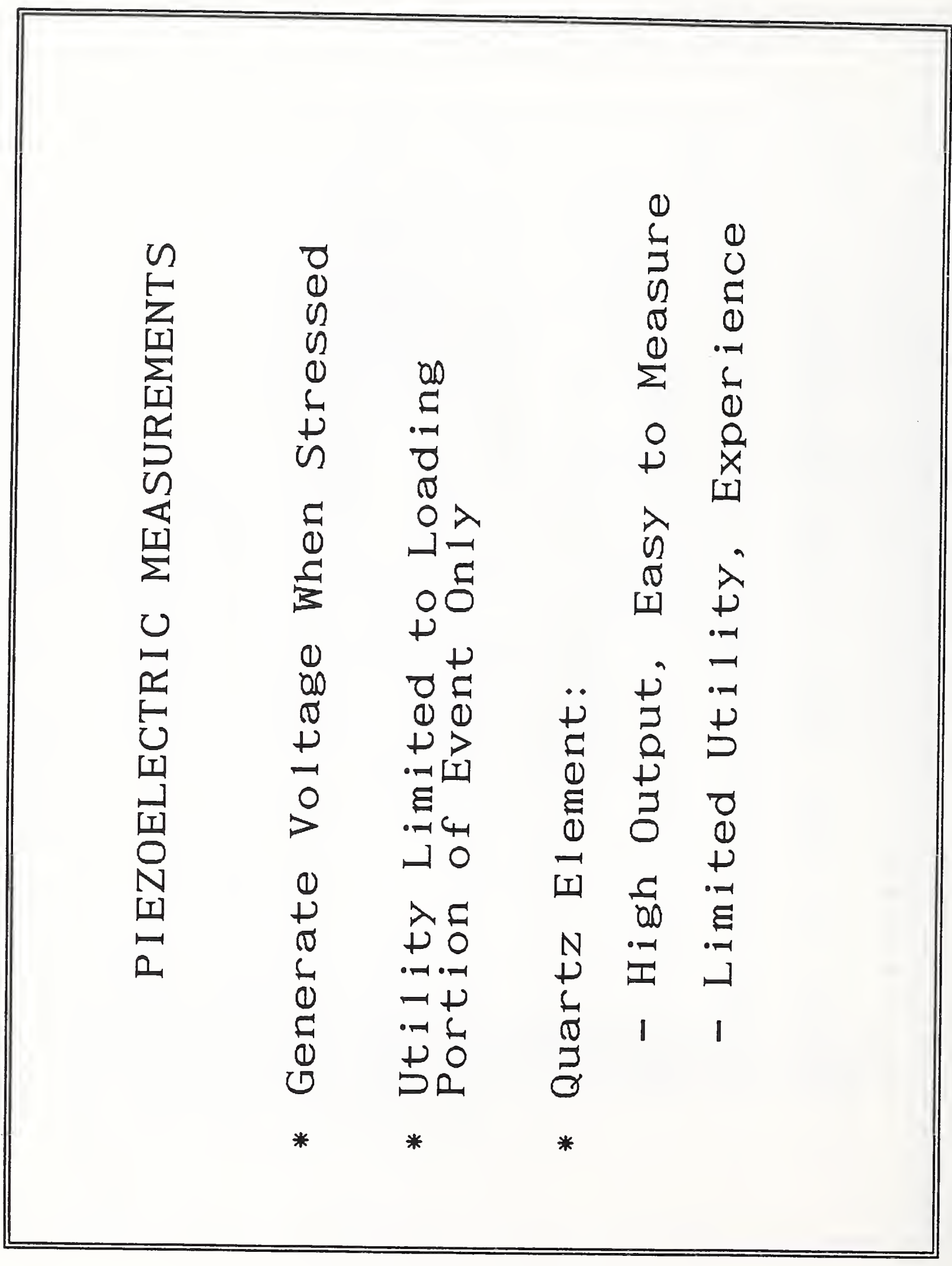




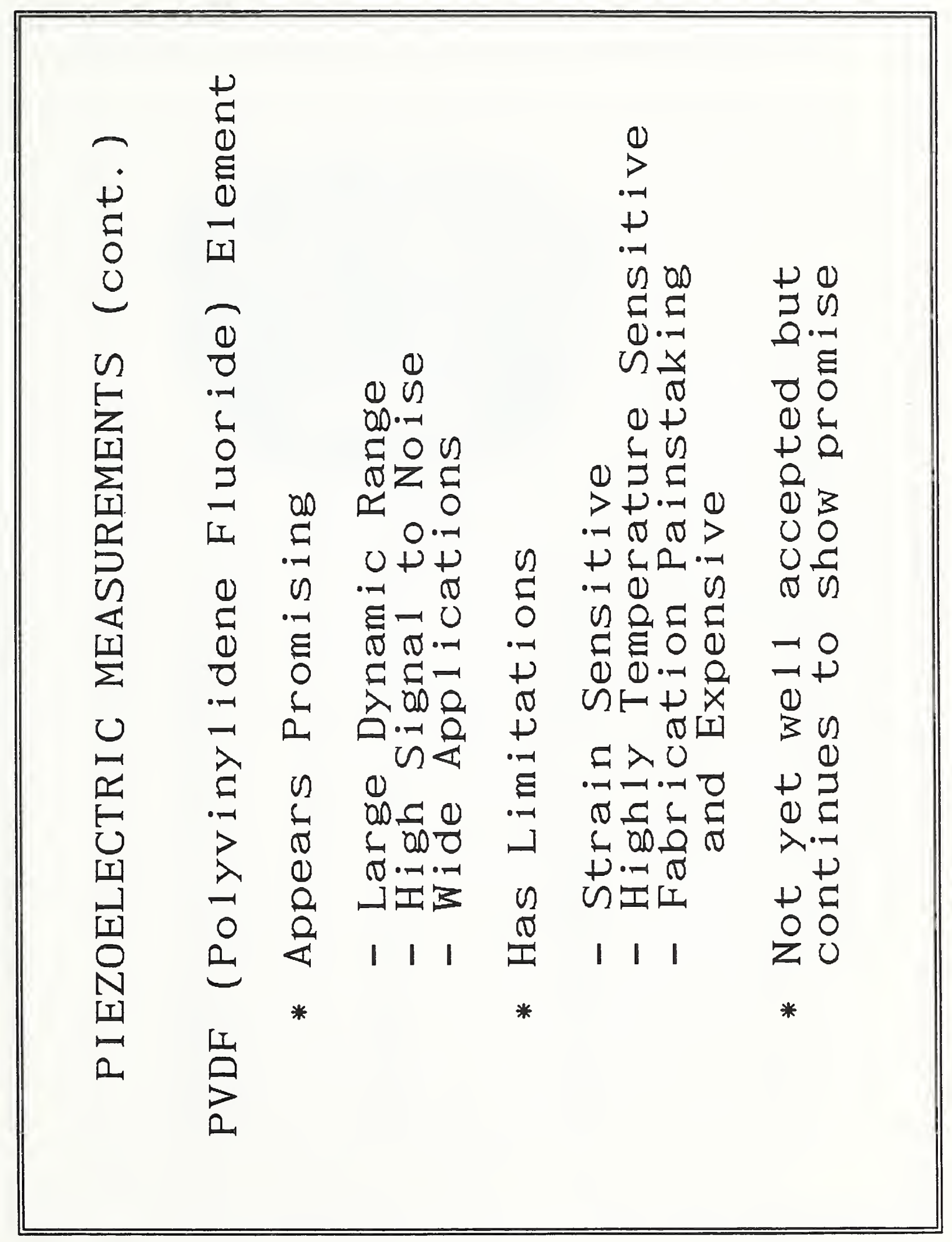




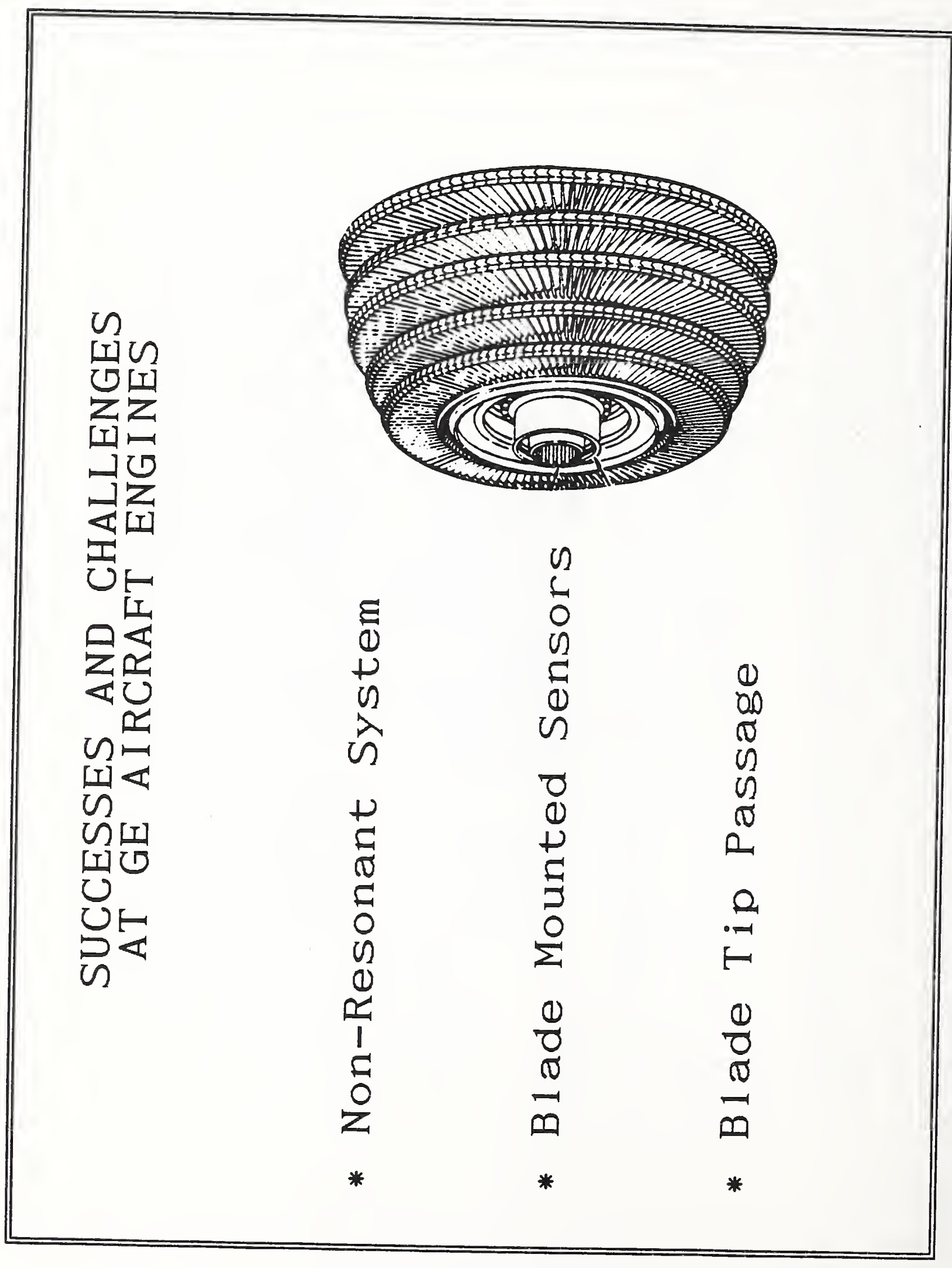




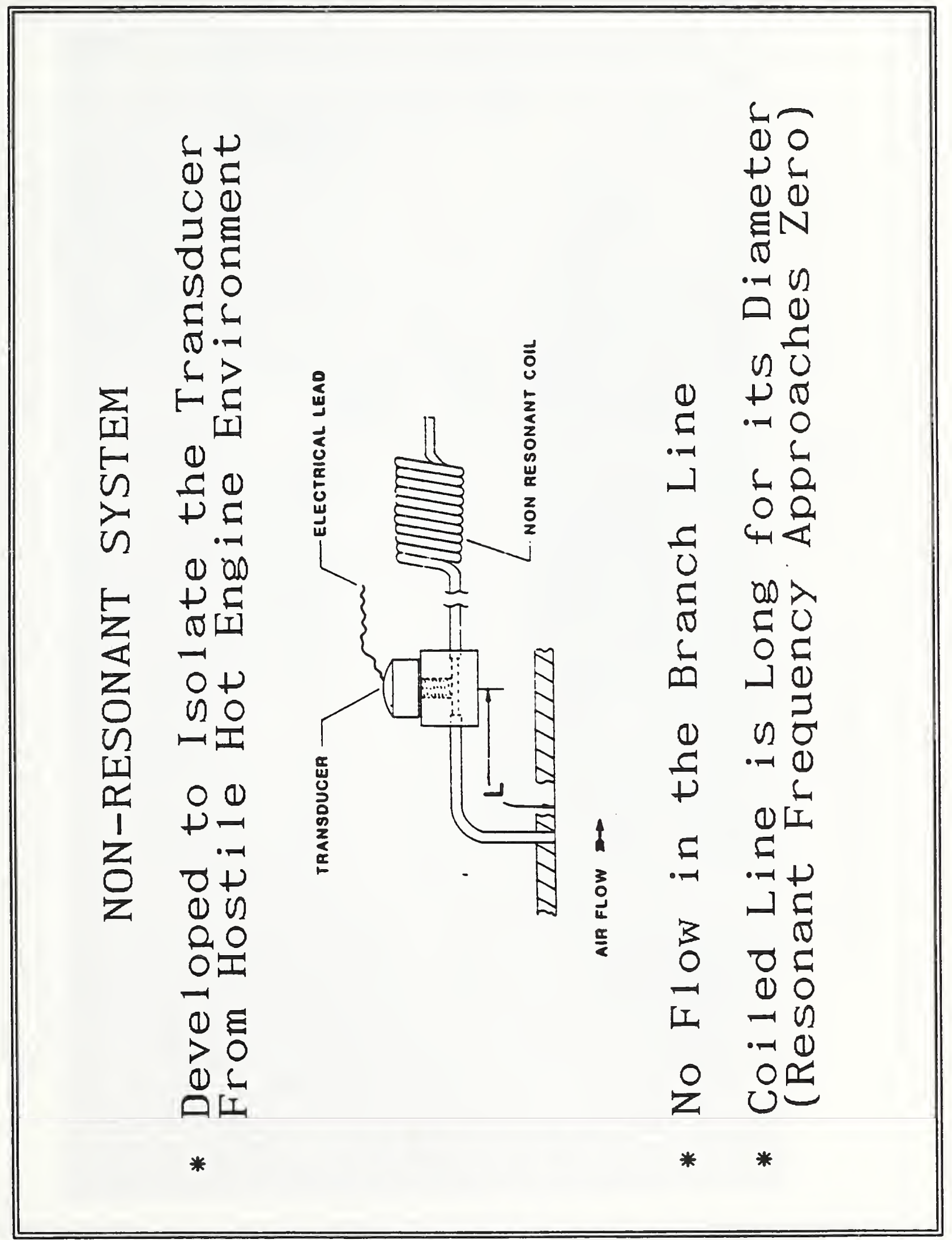




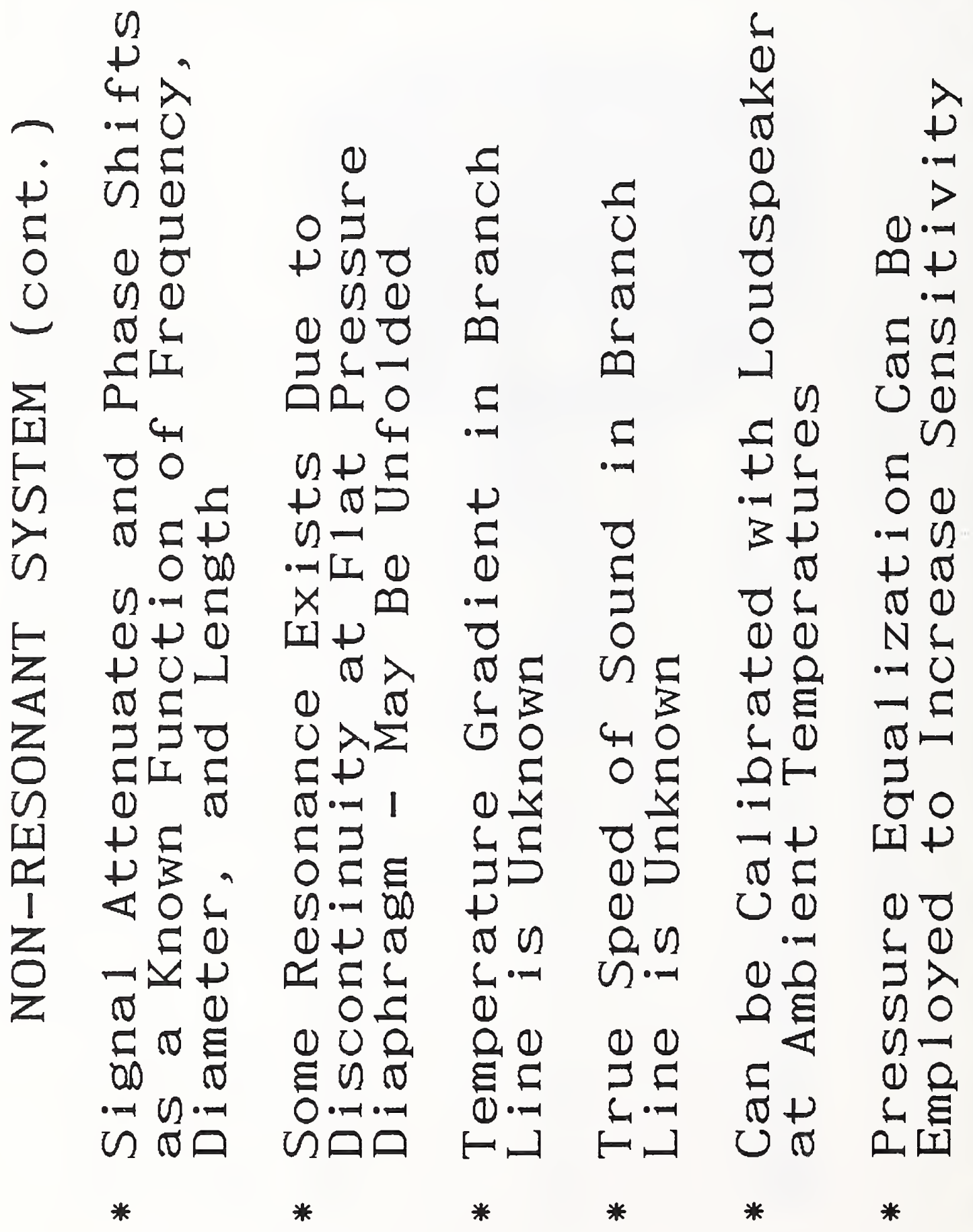




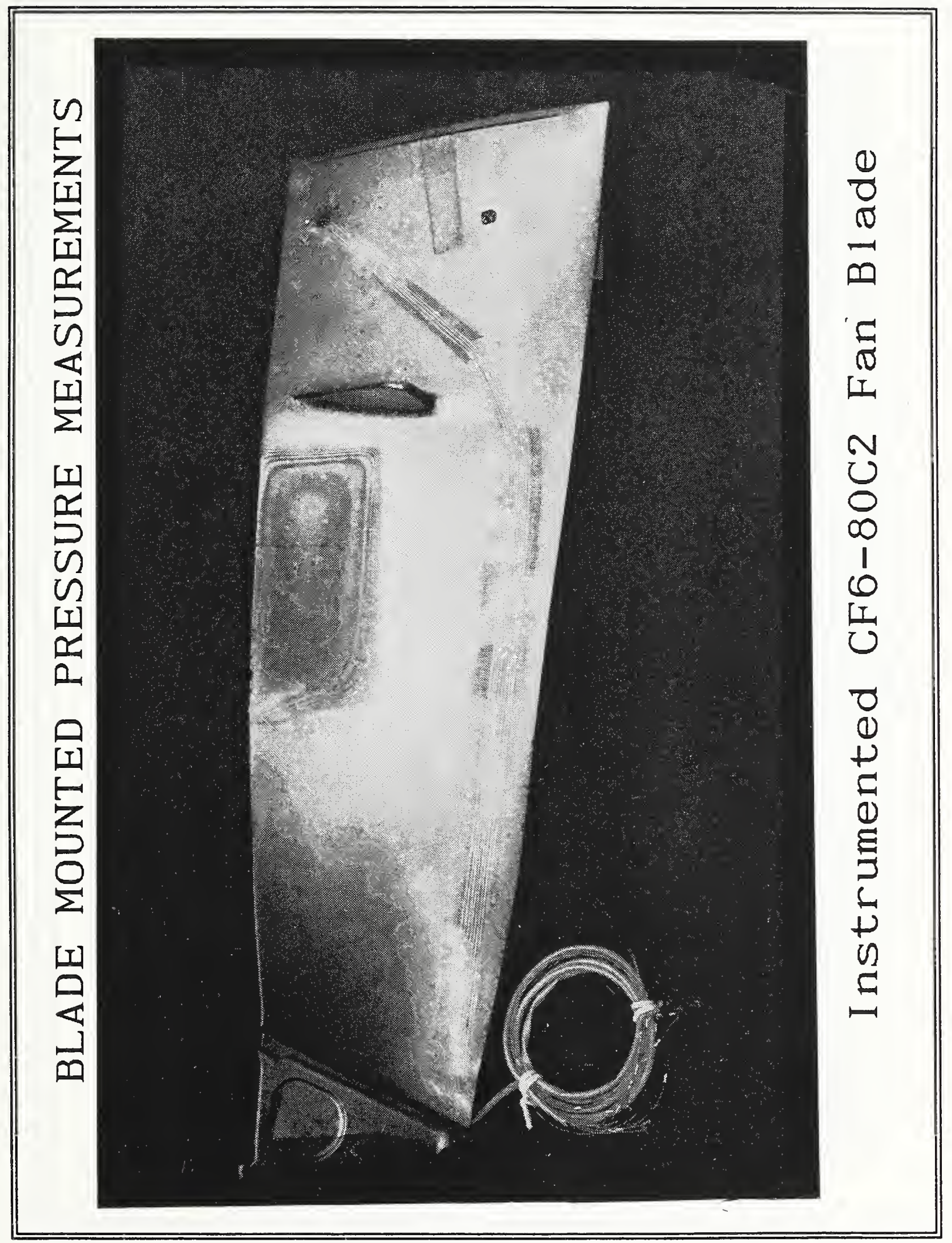




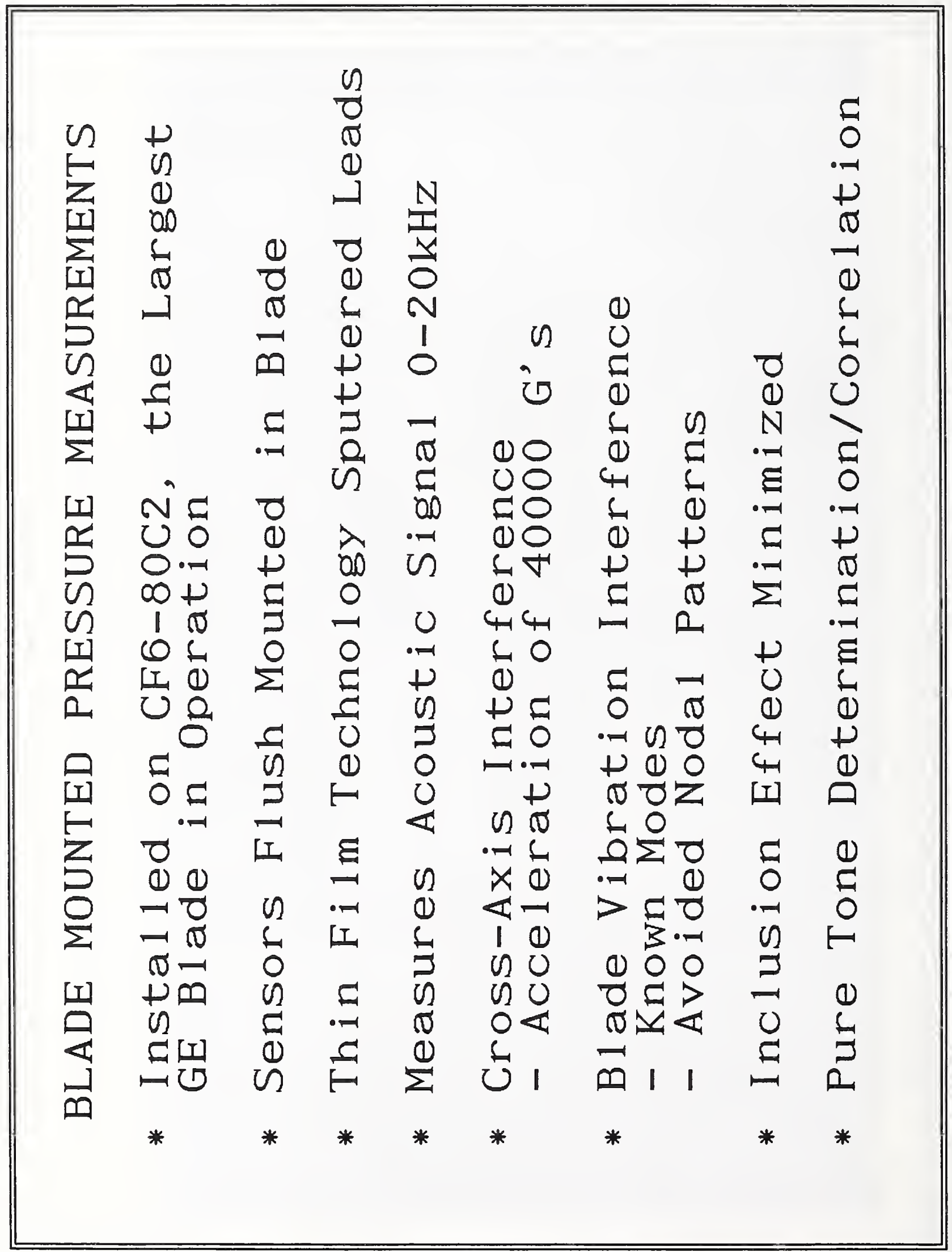




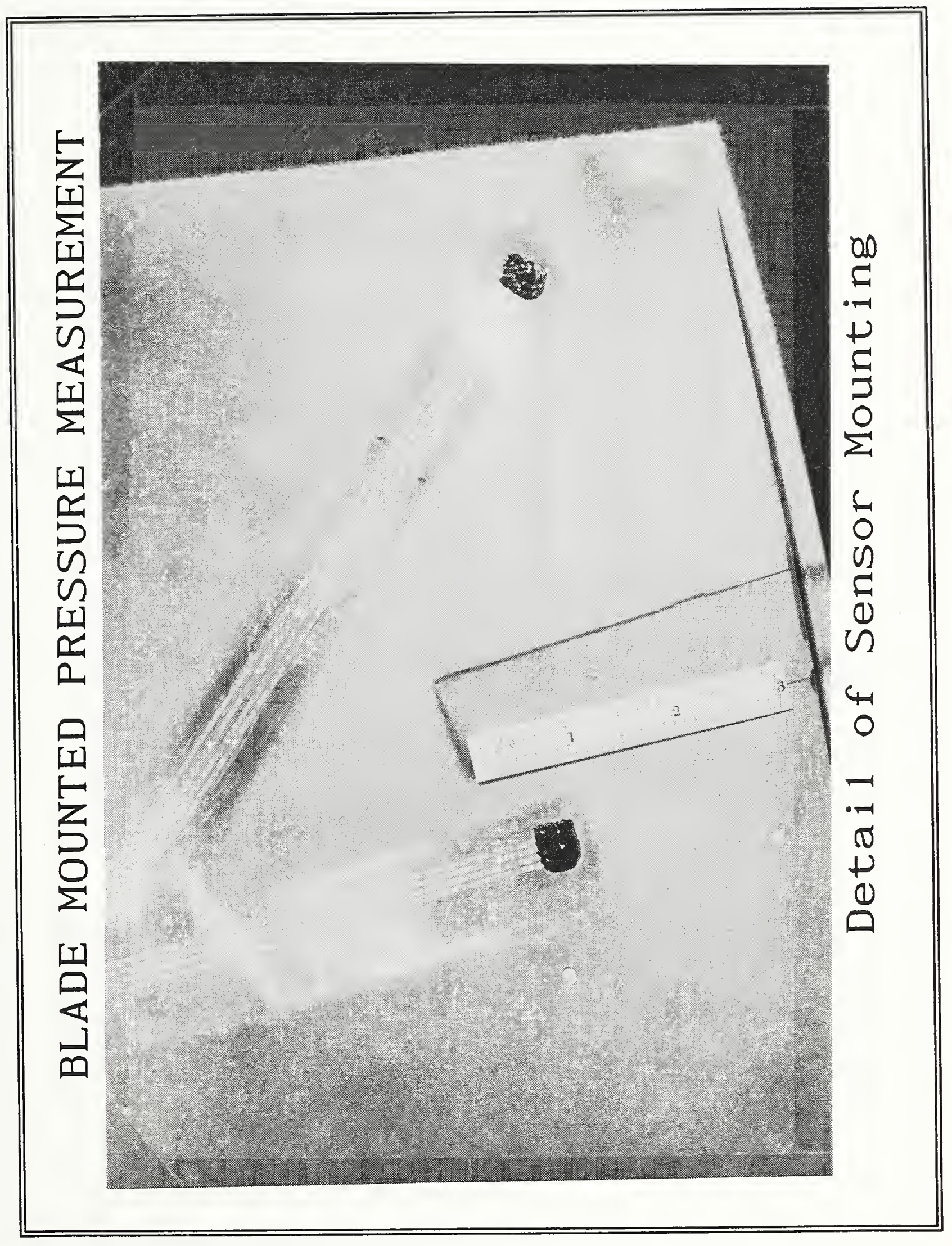




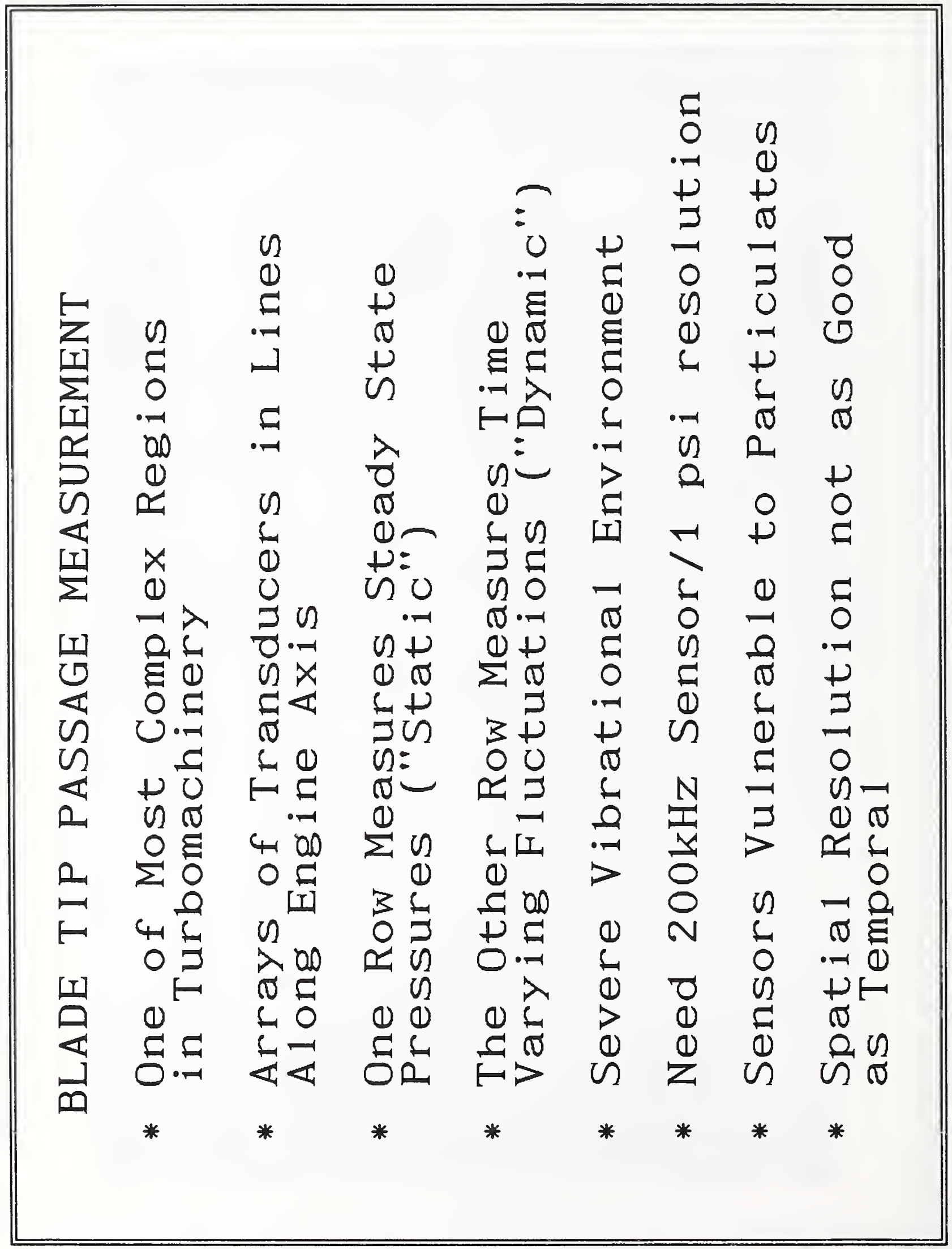




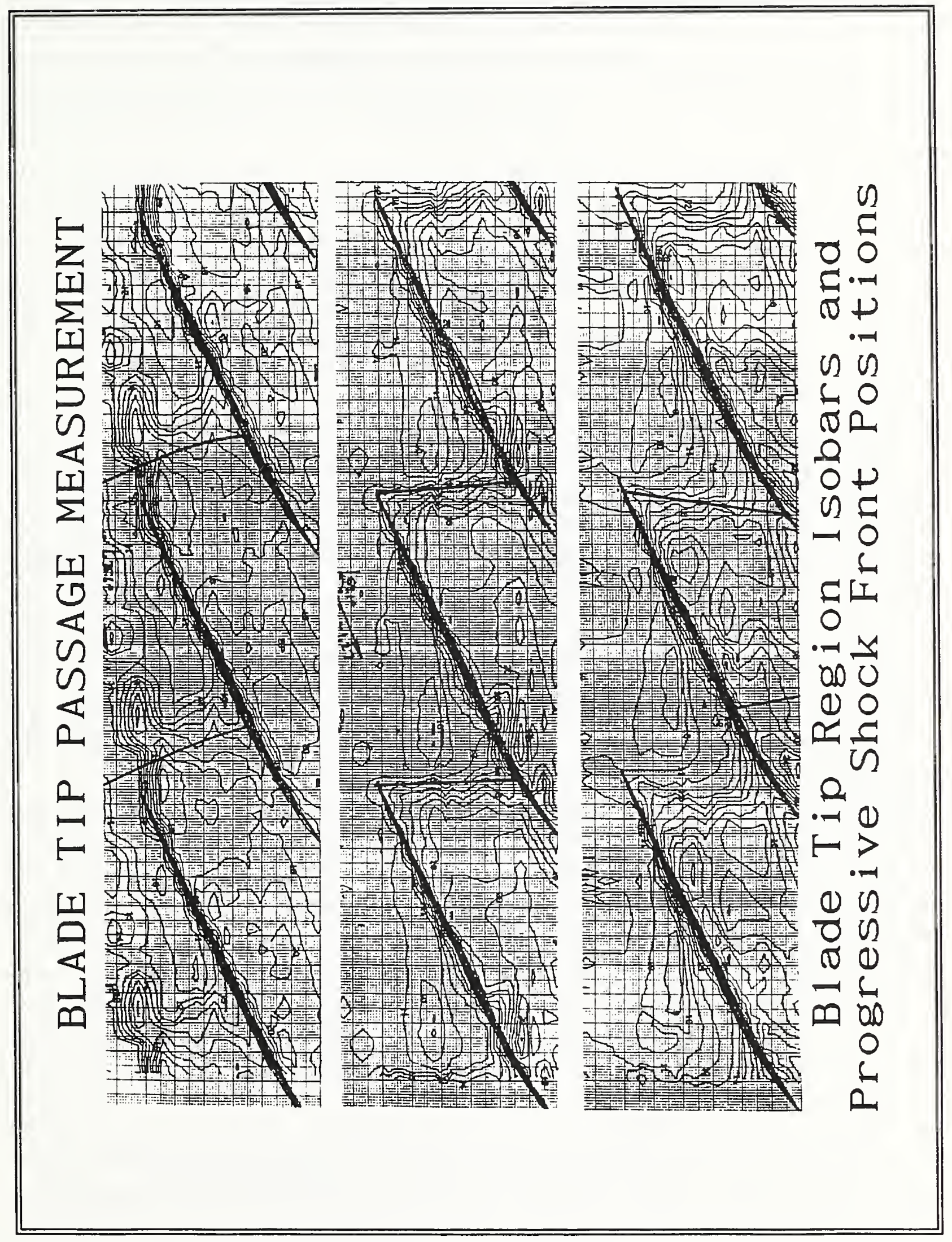




\title{
EXPLOSION SHOCK MEASUREMENTS
}

(UNCLASSIFIED)

\author{
RONALD B. TUSSING
}

\section{NAVAL SURFACE WARFARE CENTER WHITE OAK LABORATORY \\ (301) 394-1187}

\section{INTRODUCTION}

There are many considerations to be made in selecting a transducer or piezoelectric gauge for underwater shock wave phenomena measurements. The accuracy of the total measurements system is the primary factor, of which the gauge is only a part. However, the total system can be no better than the gauge or transducer selected.

This paper addresses the characteristics of tourmaline, the Naval Surface Warfare Center or NAVSWC (Navy) tourmaline gauge and its response, and the methods used to determine the response. Tourmaline has been the transducer of choice for over 40 years, and still remains such. The NAVSWC gauge has been used to measure the data to characterize all of the Navy's explosives for over 20 years, which are archived at the NAVSWC White Oak Laboratory. The NAVSWC gauge has been made available for data comparisons internationally to over a dozen countries, as well as to national laboratories and companies. The NAVSWC gauge has been used on innumerable tests: charge weights ranging from a few grams to 20 tons; test sites including pressure vessels, ponds, quarnies, rivers, and the deep ocean; and environments ranging from the tropics to the arctic.

\section{NAVSWC TOURMALINE GAUGE}

\subsection{Characteristics of Tourmaline}

Tourmaline is a hard natural crystal that is bulk or hydrostatically sensitive. Its piezoelectric constant is high enough to be useful and is constant over an adequate temperature range. 
A survey of piezoelectric activity of minerals by W. L. Bond (1948) revealed that only quartz and tourmaline were practical for extensive piezoelectric work. Since then, lithium niobate and PVDF or PVF2 have shown some promise, though PVDF is presently characterized well in only one dimension and its response is nonlinear. Some of the characteristics for tourmaline are:

Piezoelectric Constant: K = 11 Picocoulombs/psi

Linear Pressure Range: 1 psi - 50,000 psi

Dielectric Constant: 7.44

Density: $190-200 \mathrm{lb} / \mathrm{ft}^{3}\left(3.0-3.2 \mathrm{~g} / \mathrm{cm}^{3}\right)$

Hardness: 7.3 (Mohs' Scale)

Sound Speed: $19,685 \mathrm{ft} / \mathrm{sec}(6000 \mathrm{~m} / \mathrm{sec})$

Piezoelectric Moduli: d31, d33

Tourmaline must be cut perpendicular to the $z$ axis or optic axis. Charge is generated on the faces perpendicular to this axis by pressure applied to these faces as well as the parallel faces or edges: $K=d 33+2 d 31$. The properties of quartz are similar to tourmaline except that quartz is not hydrostatically sensitive, and its edges must be constrained or protected from the shock wave. Tourmaline has the hydrostatic sensitivity advantage and does not require any mechanical shielding to permit a certain direction of strain. This avoids the accompanying problems of diaphragms, mechanical resonances, reflections and complexity.

\subsection{History of the NAVSWC Gauge}

The NAVSWC (Navy) gauge is fabricated and calibrated currently at the R15 Dahlgren site. The gauge evolved from the Wood's Hole gauge used by R. H. Cole. A contingent of scientists from Wood's Hole involved with underwater explosions research moved to the Naval Gun Factory in Washington, D. C. in 1949, and then to Indian Head, Maryland in about 1950. Some of this group moved to the Naval Ordnance Laboratory (presently NAVSWC) in White Oak Maryland when it opened; however, all testing was done in the Potomac River at Indian Head site. The best coating used at this time was bees-wax and it became the standard internationally. Many gauges were purchased from a small company Crystal Research, although gauges were always fabricated in-house, and all gauges were calibrated in-house using a dead weight tester. When Crystal Research went out of business in the 1960's, the Naval Ordnance Laboratory bought all their equipment and tourmaline. Some of the tourmaline went to UERD(Underwater Explosions Research Division) of DTRC(David Taylor Research Center). When their gauge person retired, NAVSWC was the only one in the U.S. fabricating an underwater tourmaline gauge. The 
NAVSWC gauge was then made available to other agencies and internationally to other countries as a test standard for comparing data, and it still is made available.

In the mid 1960's R. S. Price and J. B. Dempsey experimented with gauge coatings to improve the variations in testing, using a statistical approach to minimize the standard deviation or sigma. The myriad of coatings tried, included various plastics, waxes, epoxies and various rubber compounds. In 1967, a gauge immersed in silicone oil in a plastic boot proved to diminish the variations noted by all of the other coatings. Final and conclusive results were reported in 1972. The problem with other coatings seemed to be that each generated a charge in an inconsistent way that added to the charge generated by the tourmaline crystals. These materials essentially changed the values of the gauge constants, inconsistently causing variations with use and time. Other materials rounded the shock peaks drastically. In the early 1970's, R. B. Tussing demonstrated a transient method of evaluating a gauge's transfer function which was more definitive and required fewer tests, than the statistical approach. The technique was not reported upon until 1982.

\subsection{NAVSWC Gauge Construction}

The tourmaline must be sliced perpendicular to the $z$ axis and then the pieces are lapped (see Fig. 1). The discs are cut with a diamond hole cutter and assembled between metal tabs. Disc sizes range from $1 / 8^{n}$ to $2^{n}$, with one to eight discs paralleled. The four disc gauge is used more in sizes of 1/4", 3/8", 1/2", 3/4", and 1". Single disc gauges are used for close-in measurements at high pressures where gauge damage is expected and high output is not desired. The assembled crystal disc elements are enameled with a high dielectric paint and mounted to an oil barrier of nylon, after the element has been calibrated using the dead weight tester which employs a quick-opening valve. The gauge element and oil barrier is then immersed in Dow Corning DC200 high dielectric silicone fluid (100 centistokes) in a Tygon boot heated and pinched closed at one end (See Fig. 2). The flattened end was found experimentally to cause less flow perturbations than a hemispherically molded end. A lighter viscosity oil $(1000 \mathrm{~m} / \mathrm{sec})$ is used than that which would match the sound velocity in water. Though the sound velocity would match better with a heavier fluid, the bubbles are nearly impossible to remove, particularly in the field. Air bubbles in the fluid cause reflections and result in erroneous data.

\subsection{Characteristics of the Oil-booted Gauge}

The gauge calibration constant KA (pico-coulombs/psi) is a constant: it is linear and stable from about 1 psi to 50,000 psi. This has been confirmed by calibration tests at NAVSWC and reported in the literature. It is reasonable to assume that tourmaline 
is linear over a much wider range. This seems to be confirmed by the excellent agreement in experimental results obtained in controlled explosion tests at NAVSWC.

Many gauges have been used and re-calibrated for a span of 15 years showing no aging effects with a standard deviation of less than 1 percent. Pyro-electric effects have been reported to be negligible for underwater tests for the signal durations encountered, even for large charges.

The booted tourmaline gauge shows no signs of hysteresis. The upper frequency response is dependent upon the gauge size or transit time of the shock wave across the gauge, while the low frequency is controlled by the input time constant of the system to which the gauge is connected. With input impedances of 100 to 300 Megohms, and cable lengths normally required by the safe distance from the charges, the input time constant is 100 or more times the duration of the shock, usually yielding a lower response of less than $0.1 \mathrm{HZ}$. The gauge rise or transit time is considered in detail in the next section.

Adequate sensitivity with good signal-to-noise $(S / N)$ ratio and an adequately small transit time can generally be achieved. Sometimes it is necessary to place a preamplifier near the gauge to meet these conditions.

The gauge's ruggedness has been proven in innumerable tests, in some tests to pressures of 50,000 psi, others where the gauge was attached to a model's steel hull that was damaged and torn, and on ship shock tests attached to the sail of an operational submarine.

Minimum distortion includes a great deal. Any measuring device of finite size will disturb the shock medium. Some initial diffraction of the shock front occurs as it passes over the gauge. Some shock reflection occurs as the shock front encounters the denser medium of the tourmaline. This also sets up oscillations or multiple reflections within the gauge discs. The Bernoulli flow around the gauge causes the initial pressure to decrease. However, this has been shown to be a negligible effect for shock waves in water up to $30 \mathrm{Kpsi}$. Diffraction effects and internal gauge reflections are considered in the next section. Gauges are used with the disc edge-on to minimize and smooth distortions. Face on gauges disturb the flow, result in internal multiple modes of vibration, and consequently may not actually shorten the rise time. The rise is more ragged and may actually be longer for gauges of four discs face-on. Testing experience has confirmed these findings. 


\section{GAUGE RESPONSE}

\subsection{The Shock Wave}

The free field underwater shock wave is generally described by the simple exponential equation:

$$
P=P_{m} e^{-t / \Theta}, t \geq 0 \quad(p=0, t<0)
$$

where, $\mathrm{P}$ is the shock wave peak pressure at any time, $\mathrm{Pm}$ is the peak shock pressure:

$t$ is time from the arrival of the shock front: and $\Theta$ is the shock wave time constant defines as the time required for the shock to decay to $P_{m} / e$, where $e=2.7183$.

This equation is a fair approximation out to one theta, but for longer times the real shock wave decay does not continue to decrease as rapidly as described by this equation. This is not important for the upper frequency response of the recordings system or to the gauge size selection. It does have to be considered in selecting the lower frequency limit of a recording system, setting the system's input time constant adequately high, and in determining the error introduced by the electronics system to measurements of energy and impulse.

\subsection{Gauge Selection}

The selection of a gauge for underwater pressure measurements generally becomes a compromise in choosing one that is physically small, and thus has a short transit time relative to the shock wave duration or time constant $\Theta$, and yet large enough to have an adequate gauge constant $(\mathrm{KA})$ to provide a recordable signal. Other factors also become important in this selection: the recording system's sensitivity, noise levels, and frequency response; gauge cable length; availability of shock resistant preamplifiers near the gauge; the physical geometry, setup and rigging, charge size, range, cable signal, desired recording time, boundary and rig reflections, i.e., the total experiment. However, there is a criterion or gauge selection that can be divorced from the above factors. This criterion is based upon the acceptable accuracy in the measurement made by the finite gauge immersed in the underwater shock wave field. 


\subsection{Selection Criterion}

The finite gauge size affects the accuracy of measurement of the shock wave peak pressure to the greatest degree. The peak pressure affects the measurement of $\Theta$ directly $\left(\mathrm{P}_{\mathrm{m}} / \mathrm{e}\right)$. Impulse is the integrated area under the curve to an agreed upon point (typically 5 or $10 \Theta$ ), and energy flux density is the integrated area under the $p^{2}$ curve to the same points (with the inverse of the acoustic impedance as a multiplier out in front of the integral or $1 / \mathrm{pc}$ ).

Both impulse and energy are rather insensitive to small errors in the shock wave peak pressure. Therefore, since peak pressure is the most sensitive and is an important parameter for explosives comparison and effects, it is selected as the measurement criterion in gauge size selection. The peak pressure recorded is the apparent peak

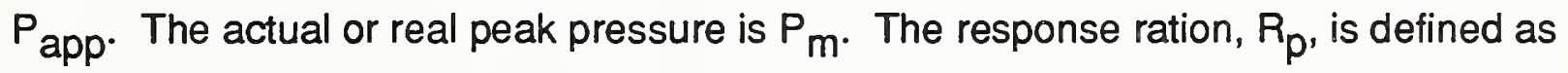
the ration of the $P_{\text {app }}$ to $P_{m}$. This ratio is dependent upon the duration $\Theta$ of the exponential shock wave and the transit time of the gauge, $t_{D}$ :

$$
R_{p}=f\left(\Theta / t_{D}\right)=P_{a p p} / P_{m}<1
$$

\subsection{Geometric Response}

The thin circular discs that make up the gauge have a geometric transit time, the time for the shock wave to cross the bare disc when facing edge-on to the shock front disregarding all flow, interference or distortion effects. A computer program was written to get the exponential plane wave response of a circular disc edge-to-edge across the disc. Separate runs were done with $\Theta / t_{D}$ ranging from $1 / 16$ to 2400 in small increments. The normalized or response ratio $R_{p}$ values were tabulated and selected values were plotted in Fig. 3 . The rise with the value of 2400 is essentially the step response.

\subsection{Actual Measured Response}

The geometric consideration is certainly a simplification of the real gauge. In fact, the gauge covering/coating, the oil boot, must be considered as part of the actual gauge. No covering/coating has been found that is transparent to the shock wave. It would have to have the same acoustic impedance and properties as the water, and yet afford protection and insulation. The diffraction of the shock front around the real gauge will also tend to make it appear larger or its transit time longer. The strain and 
signals induced in the tabs for connection to the cable will also make the gauge look longer and lengthen the transit time. these strains may also cause transverse modes of vibration or oscillation.

If the gauge coating has little deleterious affect, then the shape of the rise of the real gauge should be controlled, or effectively be the rise of the bare crystals themselves. Consider the real gauge to be a black box with a transfer function equivalent to the bare gauge. If the actual signal recorded is the same shape as the signal predicted by computation except for the duration, then the coating has done nothing but make the real gauge look like a larger diameter bare gauge.

High frequency response recordings were made with an analog oscilloscope system using fast sweeps for gauges of size 1/4-inch. The shock peaks on the film records for the $1 / 4$ inch gauges are about $4 \mathrm{~cm}$ in height. These were digitized and plotted to an expanded scale five times larger $(20 \mathrm{~cm})$. The 1/2-inch gauge records were recorded on a a Nicolet digital scope and plotted to the same scale. Some records have been obtained for 1/8-inch gauges from large shaped charges and tiny cylinders. The few records appear to have the same shape as those for the $1 / 4$-inch and 1/2-inch gauges, as they should. However, these have not been plotted to this expanded scale.

The results of these plots will be discussed in detail in the next section. It is sufficient to say that the plotted real gauge rise shapes were excellent matches to the computed geometric. It should also be noted that the gauge constant of the oil-booted gauge was the same as for the bare gauge, and that the standard deviation of a set of measurements was reduced with use of the oil-booted gauge. Separate pressure pot calibrations of the calibration constant KA for the booted gauges and then their bare crystal elements were identical. The rise responses of an oil-booted gauge and a wax-covered gauge subjected to an underwater shock are compared in Fig. 4. Notice that the wax not only slows the rise time but changes the wave-shape by rounding and smoothing.

\subsection{Response Comparisons}

The measured transit time of the oil-booted gauge is about half again as long as the computed geometric transit time of the bare gauge element alone, i.e. the time for the shock wave to traverse the diameter of the bare gauge element: $t_{D}$ (measured) $\cong$ $1.5 t_{D}$ (geometric). Therefore, in order to compare recorded gauge response with the 
computed geometric response, the recorded responses were reduced by their respective transit times, i.e. an actual gauge rise was reduced by its measured transit time.

The measured $\Theta$ was divided by the measured $t_{D}$ to get the values of this ratio. The computer runs of geometric or computer responses were identified by this same ratio.

The comparisons of reduced actual response and geometric response were plotted for matching values of $\Theta / T_{D}$. The vertical scale for pressure response ratio was also normalized, thus, the ordinate $R_{P_{n o r m}}$.

Many fast rise recordings have been made of various size explosions and compositions. Typical charge weights were: $1 \mathrm{oz}, 1 \mathrm{lb}, 5 \mathrm{lb}, 10 \mathrm{lb}, 15 \mathrm{lb}, 50 \mathrm{lb}$, and $1300 \mathrm{lb}$. Compositions were: C-4, Pentolite, TNT, HBX-1, PBXN-103, and Minol. The ranges of $\Theta / t_{D}$ for these were 5 - 90. A limited number of fast nise recordings are shown in Fig.'s 4 - 6, for 1/4-inch gauges. The expanded and reduced plots for a 1/4inch, and a 1/2-inch gauge digitally recorded, are shown in Fig.'s 7 - 8. Note how closely the actual rises for the real gauges match their appropriate geometric curves.

This comparison of the actual rise shape to that predicted for the bare crystals by the geometric response proves to be an excellent technique for evaluating gauge coatings and various other configurations. This technique along with the comparison of calibration constants (KA) for the bare crystals and for the covered gauge defines the characteristics of the completed gauge.

The small perturbations or oscillations on the plotted rises of Fig.'s $7-8$, are seen in the actual oscilloscope records, Fig's $4-6$. These are the internal reflections of the shock front within the tourmaline crystals. The shock speed within tourmaline is about four times faster than in water. Note that there are inflection points at about the 1/4 points. The reflections in the crystal would be expected to be damped oscillations with the same S-shaped rise and fall.

Note also the damped oscillations after the peak. These are at lower frequencies that correspond well with the gauge size and the shock speed in the water. They are probably mechanically induced stresses or reflections off gauge tabs or connections. Further investigations with modified gauge constructions would shed more light on these conjectures. Whatever the source, the oscillations are part of the real response 
of the gauge; the frequency of oscillations is directly related to the crystal size and the shock speed in water.

\section{THE NAVSWC GAUGE IN USE}

The NAVSWC oil-booted tourmaline gauge has been in use for over 20 years on hundreds of underwater explosion tests of various types and weights of charges and weapons. The gauges have been in close proximity to, mounted on and within models, ships, and submarines. Tests have been conducted in fresh and salt water with a myriad of thermoclines and sound velocity profiles. Tests have been conducted in small chambers, ponds, quarries, rivers, bays and in the ocean at a multitude of locations and environmental conditions. The gauges have been used by the NATO nations for a means of data comparisons and by over a dozen other countries, as well as by many national laboratories and companies.

The gauge characteristics have been well defined and confidence in the results have been demonstrated repeatedly. In fact, most of the current underwater explosion theory and the Navy's archives have been based upon the analyses of data measured with these gauges. Before one can believe and utilize data recorded under highly refracted environmental conditions or highly reflected conditions, a gauge's response must be well documented for the more standard free field tests; these would be tests where the exponential nature of the shock wave and bubble pulse are not interfered with by reflections from the surface and the bottom, or from ships or targets near-by, or from other boundaries.

\subsection{Explosive Comparison Fresh Water Tests}

Free field (unbounded) measurements are the more conventional measurements used to characterize different types of explosives and to compare new ones with standards and others. Generally four gauges at each recording station spaced logarithmically at four ranges are required to get the statistics and accuracies necessary for such comparison tests. Some focus has been placed on increasing the energy in the bubble pulses in the past few years, requiring integration under the bubble pulses. Thus, high gain with good low frequency response $(.01-0.1 \mathrm{HZ})$ must be achieved along with confidence in the gauge's performance, to faithfully record some of the "odd" and unexpected wave-shapes. Fig. 9 shows the total record with the shock wave and bubbles compressed in time, and then the shock and each bubble pulse expanded in the following views. 


\subsection{Model Tests}

Model tests typically require that the gauges be mounted near or on the model and sometimes inside flooded compartments that are air-backed. The reflections and resulting pressure waves cannot be predicted and can be almost anything (Fig. 10). The point is, that without confidence and experience in a gauge's performance under more controlled testing, one cannot differentiate gauge characteristics or problems from the "real" phenomena. This could result in erroneous interpretation and damage theories. A recent example emphasized the importance of this, when several finite element codes predicted "after-the-fact" radically different results with their damage explanations without regard to the data. The measurements were then the only common link that at least had to be matched by the code before further extrapolations of "what happened" could be made or believed by a code. 


\section{SUMMARY AND CONCLUSIONS}

The NAVSWC oil-booted gauge:

- has been well characterized and has demonstrated repeatable and consistent response under all kinds of conditions and types of tests;

- has been the standard gauge for recording all of the Navy's archival data and similitude parameters;

- has been made available at cost as a standard for comparison both internationally and nationally;

- is rugged, reliable, easily fabricated, calibrated and refurbished;

- is linear, bulk or hydrostatically sensitive and is omnidirectional;

- effectively does not change the amplitude response of the bare crystals;

- appears to be half again as large as the diameter of the crystal, extending the response time to about 1.5 times the geometric rise;

- is easily impedance matched to instrumentation systems, the high frequency response determined by the transit time of the shock front crossing, and the low frequency or decay response determined by the input or over-all time constant of the system;

- can simultaneously cover extremely wide dynamic pressure ranges simply by paralleling several channels of instrumentation at various gains across it;

- can be used with an in-line preamplifiers close-in to increase the $S / N$ ratio, with the resulting limitation in dynamic pressure range caused by the preamplifier;

- and remains as the transducer of choice for underwater explosion pressure measurements. 


\section{BIBLIOGRAPHY}

Cady, W. G., Piezoelectricity (McGraw-Hill. 1946).

Cole, R. H., Underwater Explosions (Princeton University Press, 1948).

Dempsey, J. B. and Price, R. S., "Reduction of Scatter in Underwater Shock Wave Measurements Made with Piezoelectric Gage", NOLTR 72-12, Feb 1972.

Frondel, C., "Tourmaline Pressure Gauges", The American Mineralogist, Vol. 33, JanFeb 1948, pp. 1-17.

Tussing, R. B., "Accuracy and Calibration Requirements of Oscilloscope Recording Systems", NOLTR 65-77, Oct 1965.

Tussing, R. B., "Accuracy and Response of Tourmaline Gages for Measurement of Underwater Explosion Phenomena", NOLTR 82-294, JUL 1982. 


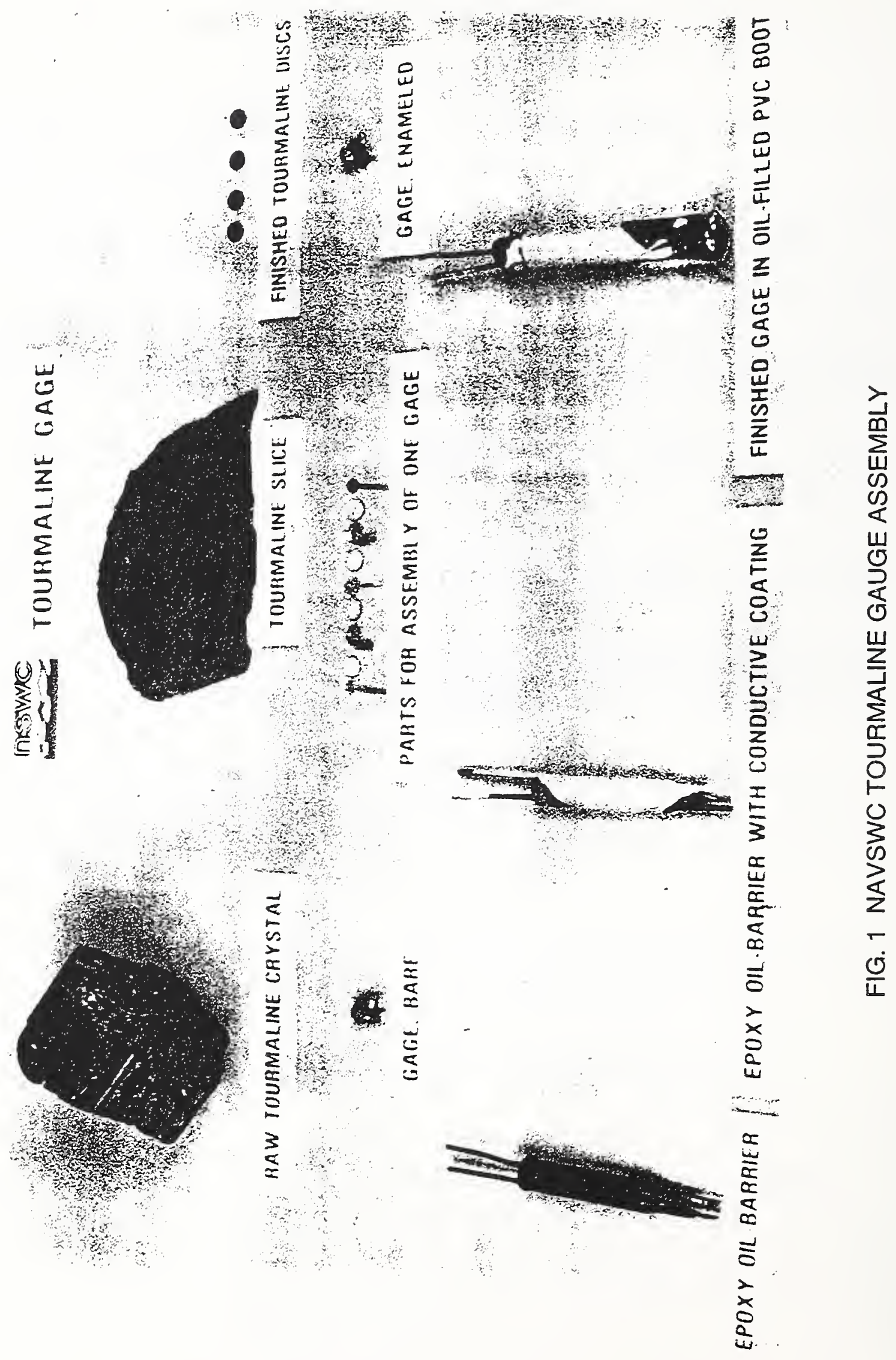




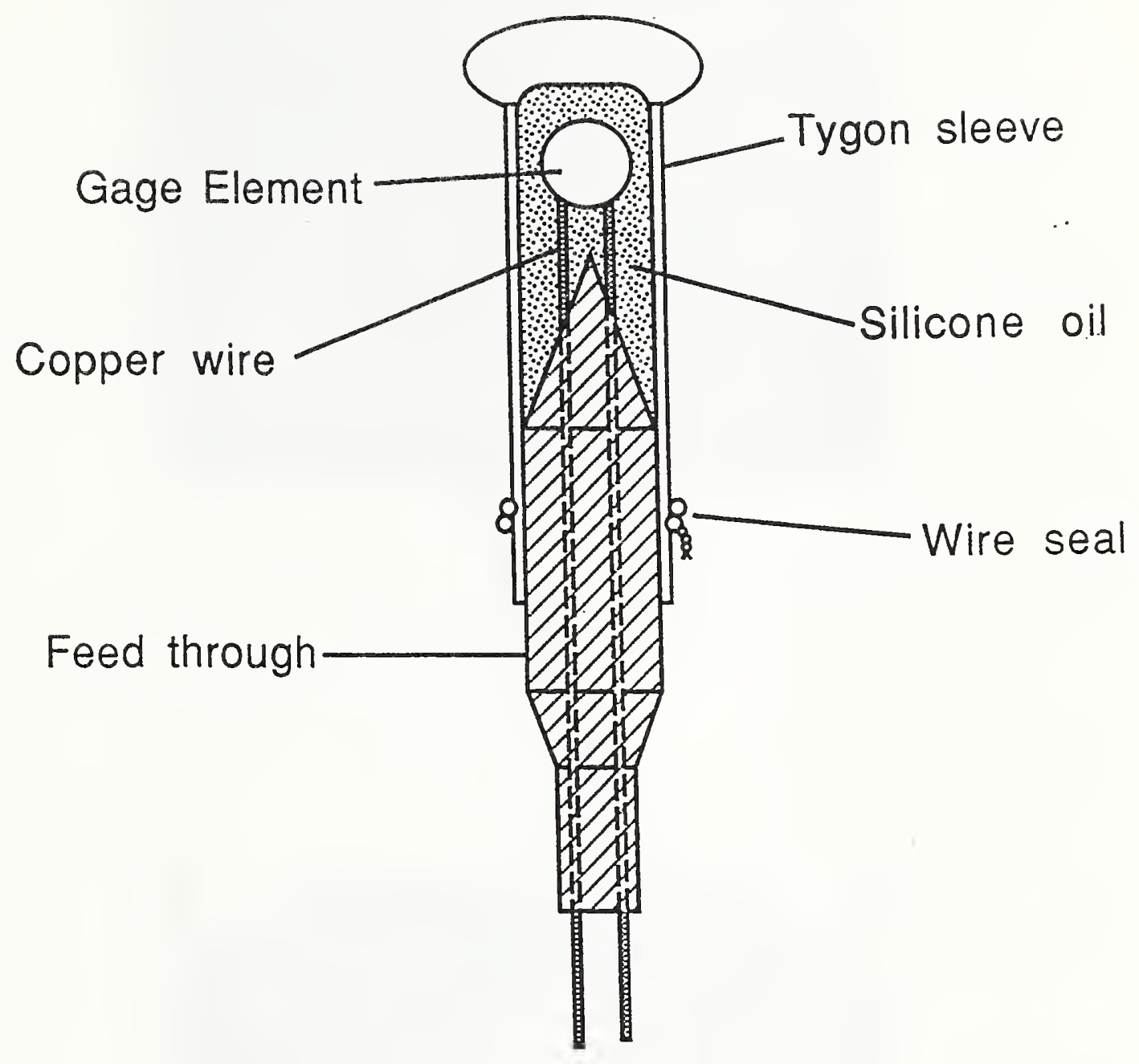

to coaxial cable

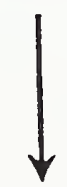

(not to scale)

FIG. 2 NAVSWC TOURMALINE GAUGE DIAGRAM 


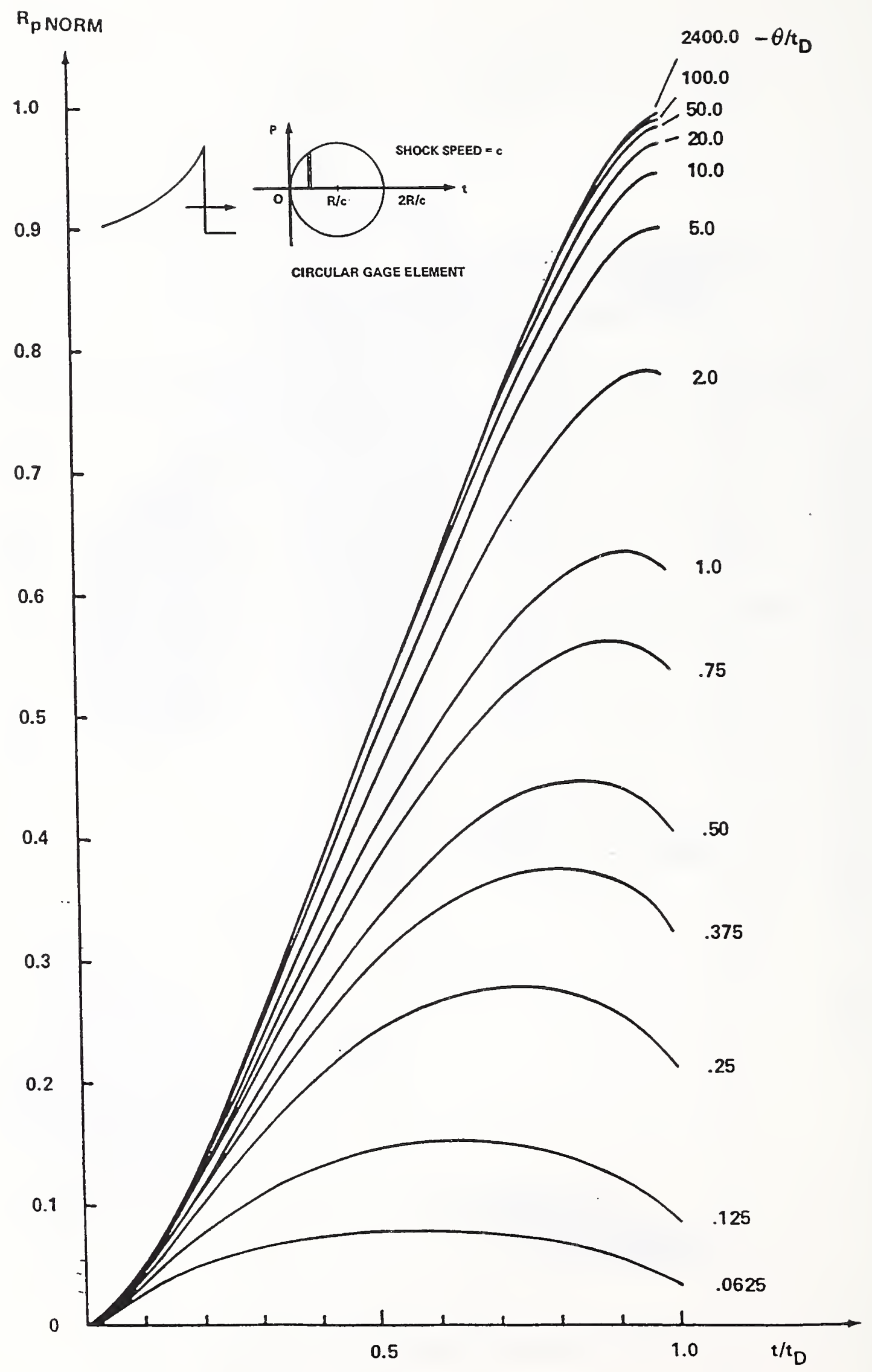

FIG. 3 GEOMETRIC GAUGE RISE RESPONSES 


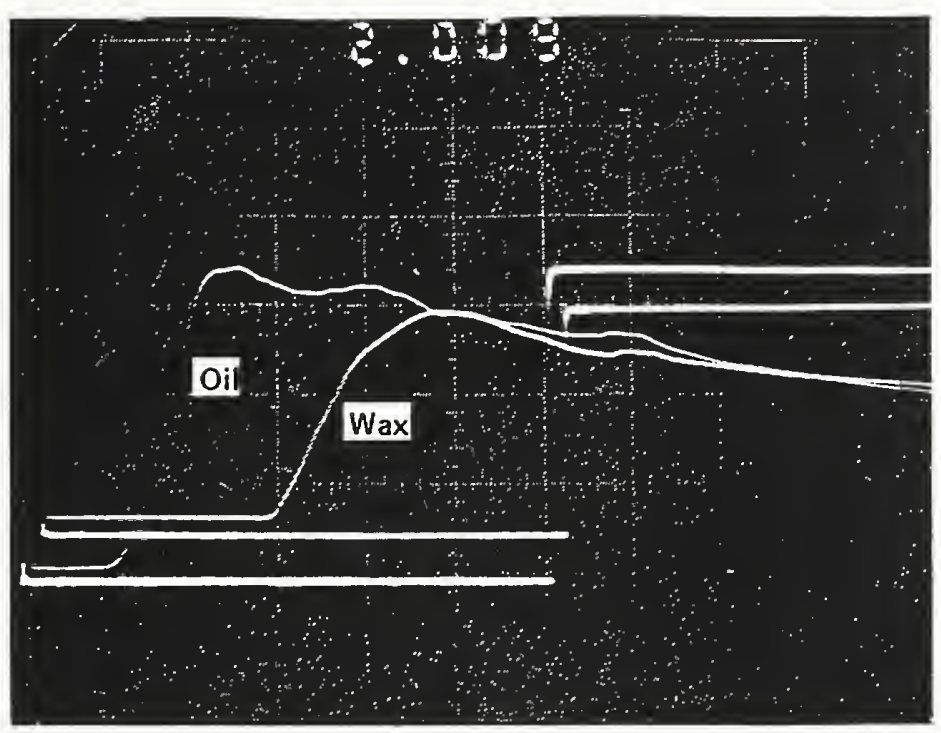

$5 \mu \mathrm{sec} / \mathrm{cm}$

FIG. 4 GAUGE RISE COMPARISON OIL BOOTED VS. WAX

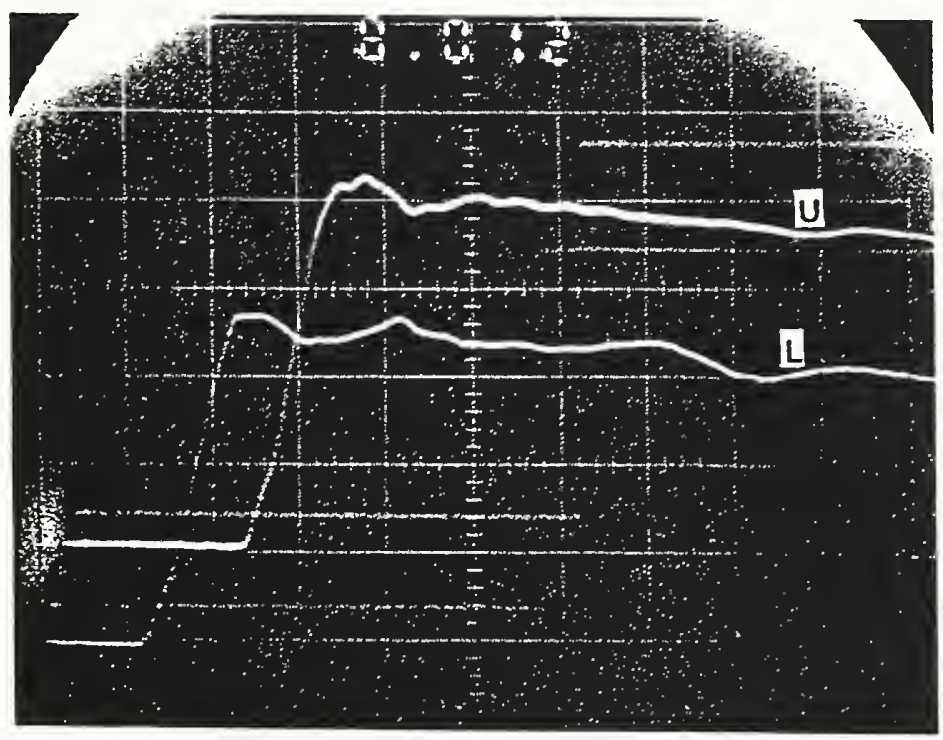

$5 \mu \mathrm{sec} / \mathrm{cm}$

FIG. 5 GAUGE RISES - OIL BOOTED 


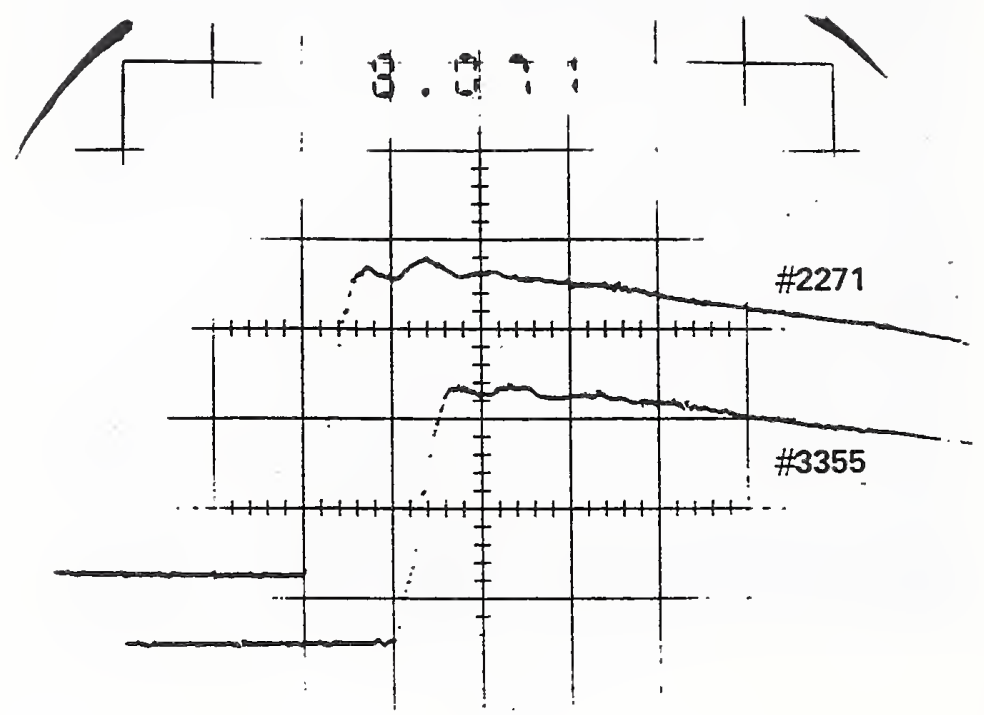

$10 \mu \mathrm{sec} / \mathrm{cm}$

FIG. 6 A GAUGE RISES

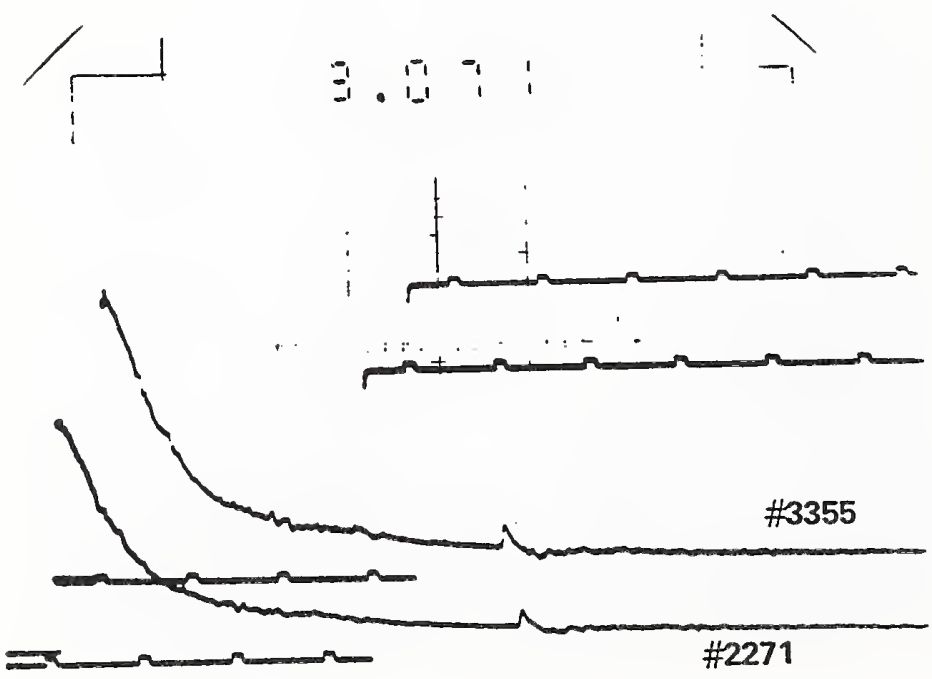

$200 \mu \mathrm{sec} / \mathrm{cm}$

FIG. 6B SHOCKWAVE RECORD FOR GAUGE RISES 


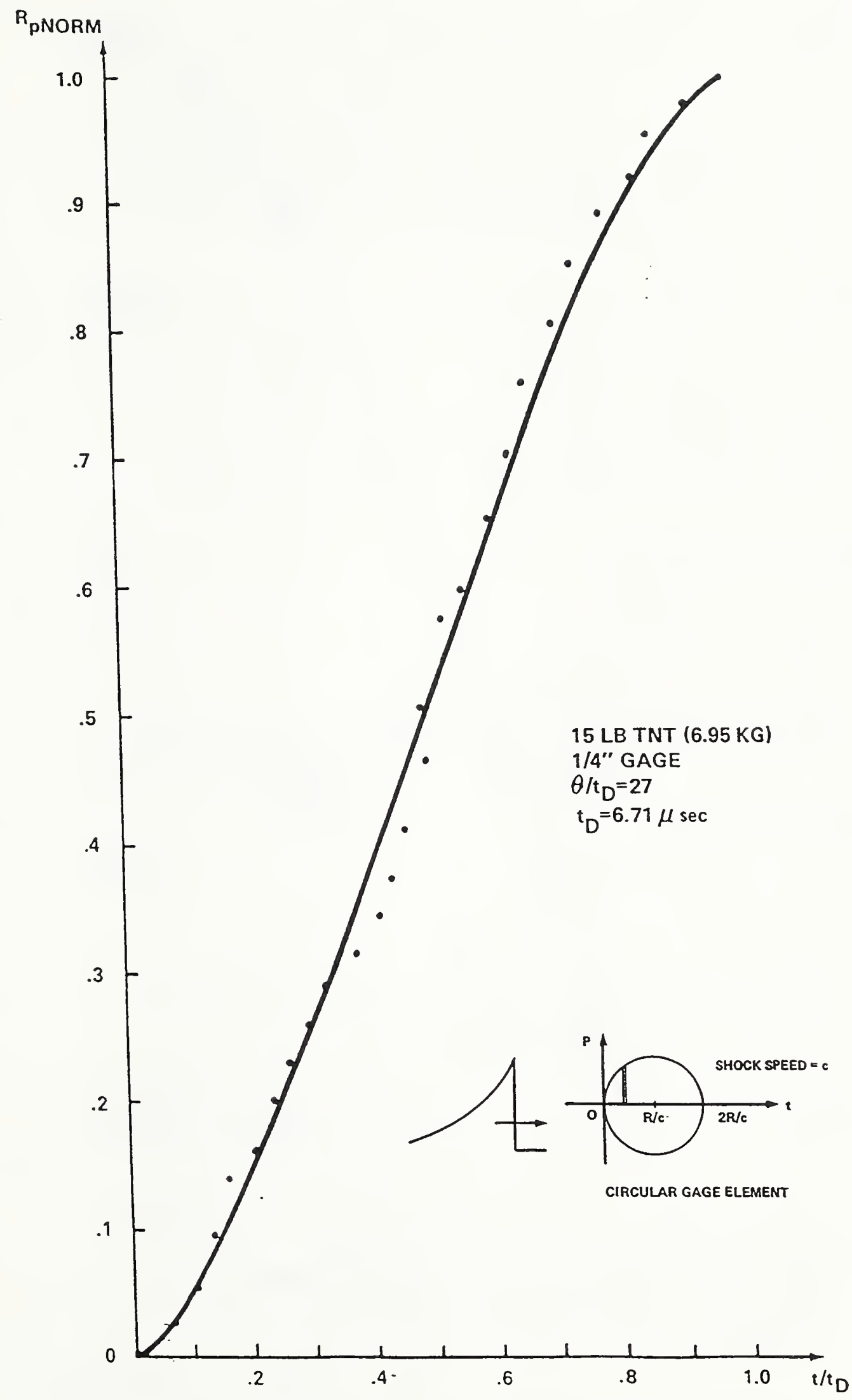

FIG. 7 ACTUAL VS. GEOMETRIC RISE 1/4-INCH GAUGE 


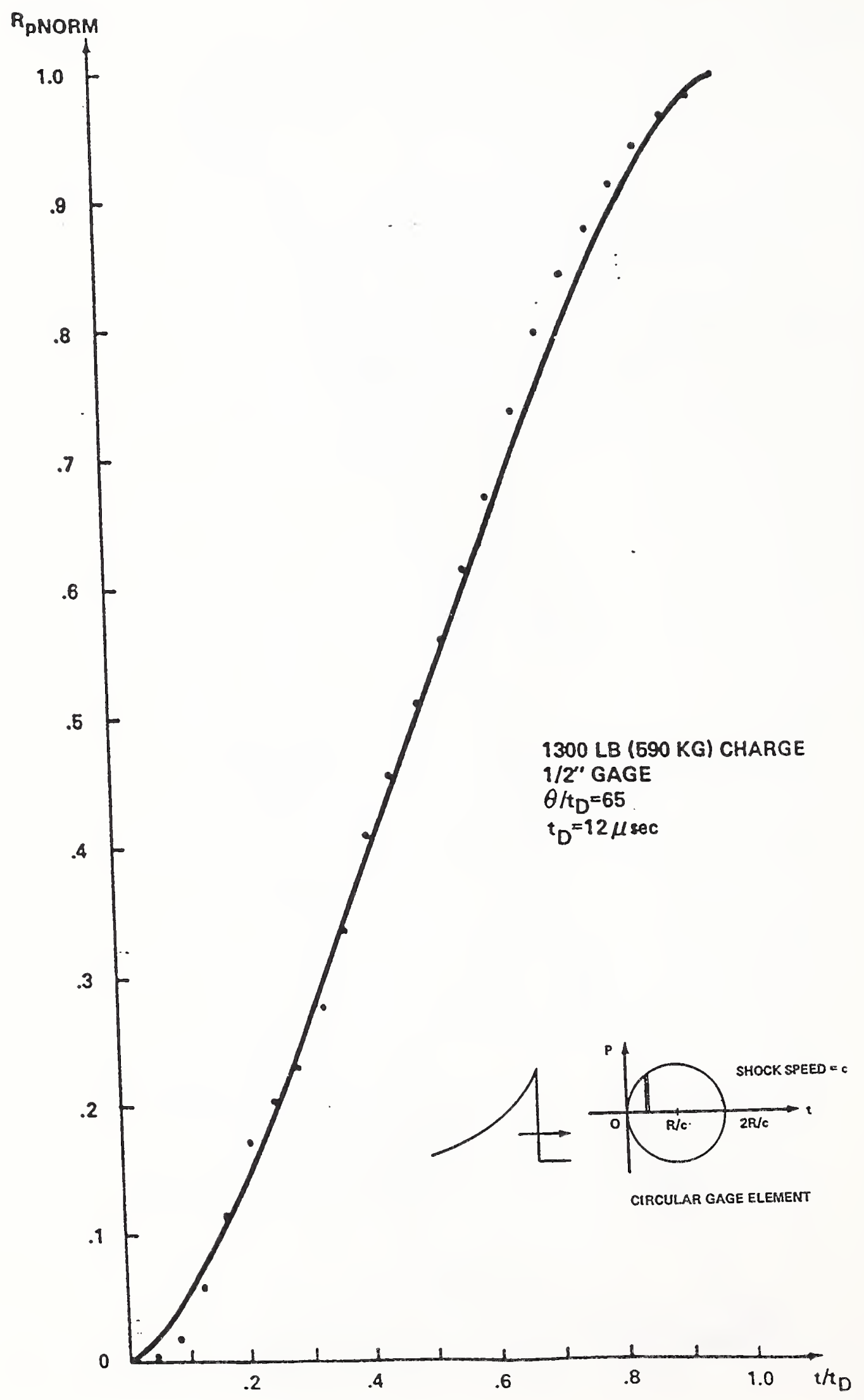

FIG. 8 ACTUAL VS. GEOMETRIC RISE 1/2-INCH GAUGE 

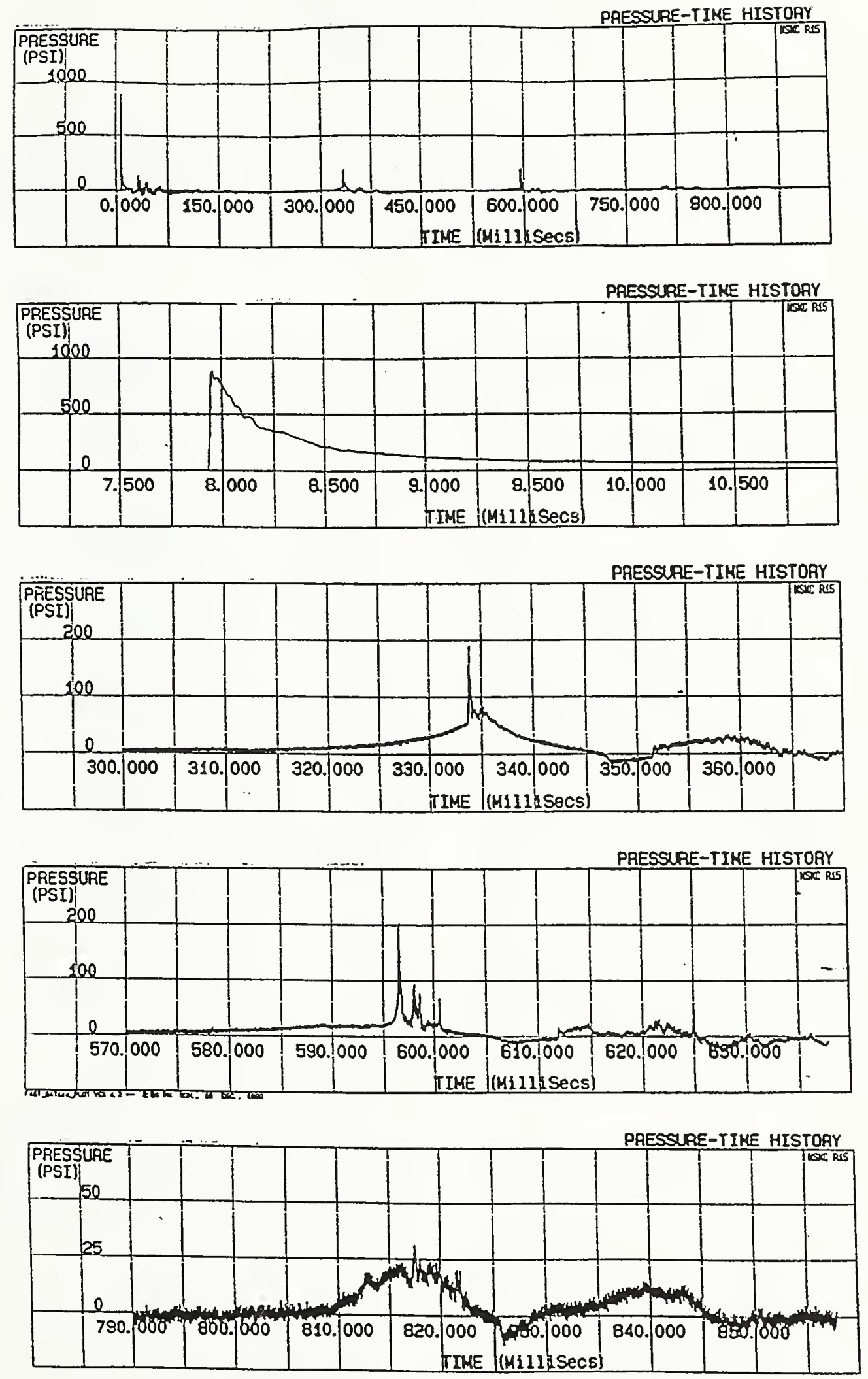

FIG. 9 SHOCKWAVE \& 3 BUBBLES EXPANDED 
PAESSURE VS TIME

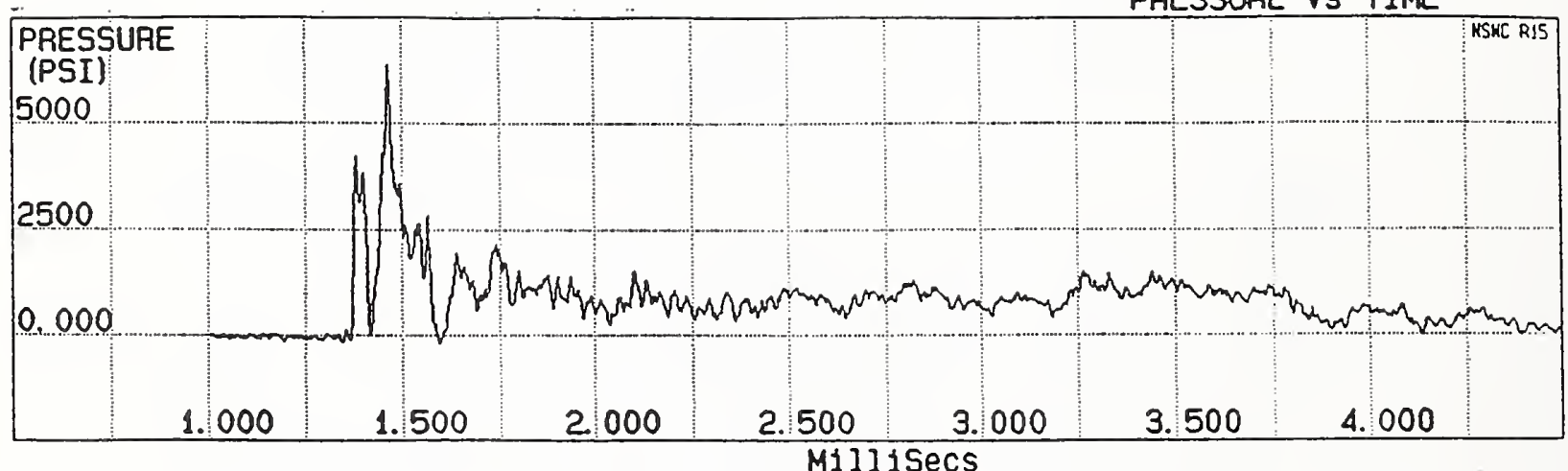

PRESSURE VS TIME

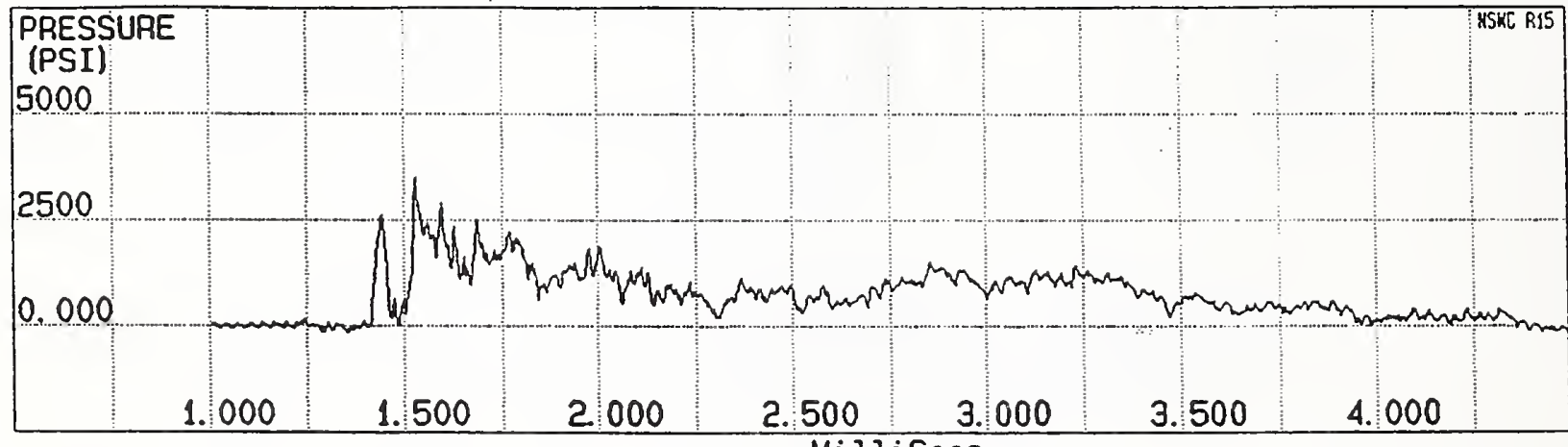

Millisecs

PRESSURE VS TIME

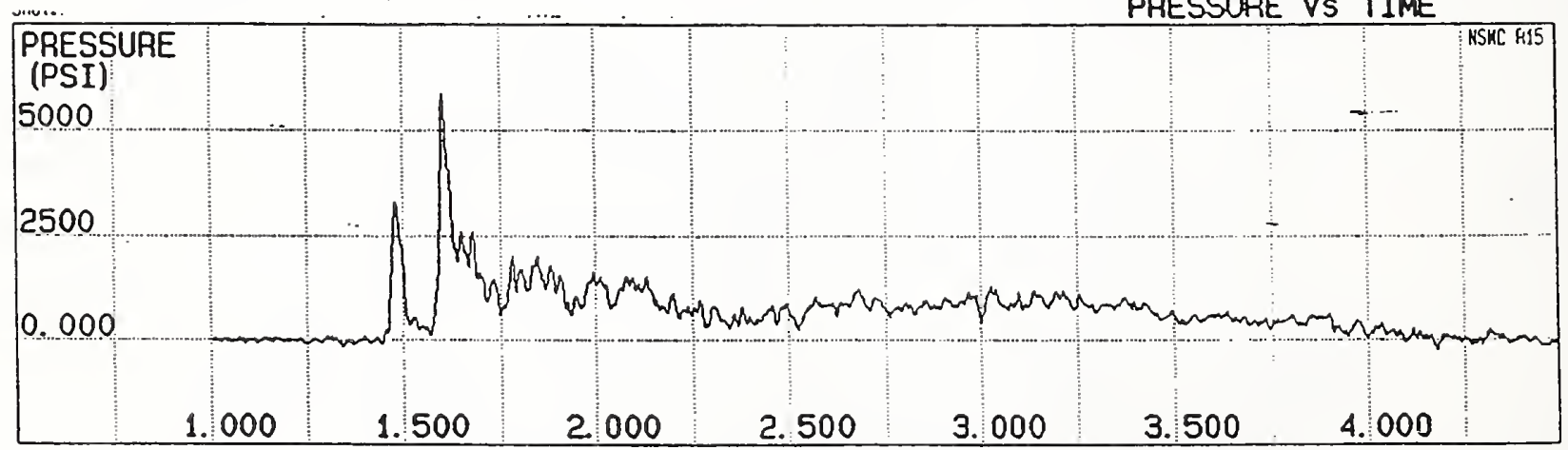

FIG. 10 MODEL TEST - INTERNAL GAUGES 


\section{ARMY DYMAMIC PRESSURE MEASUREMENTS \\ By John M. Ball \\ U.S. Army TMDE Activity \\ Redstone Arsenal, AL}

ABSTRACT: This paper briefly describes dynamic pressure measurements in the Army, tests performed, ranges of interest, and transducers. Army calibration program and traceability requirements are outlined along with measurement concerns. Several actual calibration systems are described. The need for a national calibration service and measurement program in this parameter is emphasized.

\section{INTRODUCTION.}

A national survey of Army dynamic pressure measurement activities was conducted in 1986 [1]. This census, while not all inclusive, identified quantities of transducers, ranges of interest, and locations at which dynamic pressure measurements were conducted. Test ranges, proving grounds, and development centers constituted the principal users of dynamic pressure transducers. While ranges of interest varied with the application, measurements from thousands of atmospheres to tens of thousands of atmospheres were then common and are currently being performed on a routine basis in a number of locations. Higher range measurements usually involve combustion generated pressures: chamber pressures, blast pressures, and so on. Lower range measurements are applied to such things as engine pressures. Products from most major manufacturers of dynamic pressure transducers and unique, Army designed and manufactured transducers are represented. Hundreds of these transducers are formally supported through the Army calibration process. The typical transducer had a range of about 6800 Atm $(100,000$ PSI), with an expected error of $1 \%$ to $2 \%$.

A study conducted in 1990 demonstrated that certain of these dynamic pressure machines generated pressures which agreed with precision static measurements to about one percent.[5] It was not possible, however, to directly validate the performance of these devices in their dynamic mode.

\section{ARMY CALIBRATION PROGRAM}

By regulation [2], the U.S. Army operates a calibration program designed to ensure the accuracy of measurements in all parameters and to guarantee the correct performance of Army test, measurement, and diagnostic equipment. The test ranges, proving grounds, RD\&E Centers, and laboratories which perform dynamic pressure measurements are supported by calibration and repair services from mobile calibration vans and sophisticated, fully equipped measurement laboratories. These activities are controlled, operated, and supported through a hierarchy of calibration laboratories, including the Army Primary Standards Laboratory, which functions as the "bureau of standards" for the Army.

Program management, engineering, and logistics for these calibration functions and for general purpose Army test instrumentation and automated test equipment are centralized in the U. S. Army TMDE Activity headquartered at Redstone Arsenal, AL.

\section{TRACEABILITY}

Calibration generally requires a measurement system with known accuracy and a test unit which is certified against this system. Regulations govern accuracy relationships between measurement systems and items to be calibrated [3]. In many cases, a transducer which has been calibrated is used as an "artifact standard" to transfer accuracy to other items. 
Periodic calibration detects changes in performance and permits correction or adjustment. The accuracy of a calibration is dependent upon a reference which may be another piece of hardware, a physical phenomena, or a process. The National Institute of Standards and Technology (NIST), is the organization in this country to which ali measurements except time are legally referenced. "Traceability" is the process by which the relationship of field measurements to NIST is documented.

Dynamic pressure measurements, however, are not directly traceable to NIST. This is the most significant issue in this parameter today. Neither does the NIST provide dynamic pressure measurement services. Without traceability, the accuracy of such measurements cannot be directly validated at any level in the calibration hierarchy, calibration support is reduced to the certification of repeatability at some inexactly known value, and the Army's testing program is seriously compromised.

Army users and calibration
operations have applied several different calibration approaches in an attempt to provide measurement services and some degree of traceability. Several will be described briefly later in this paper.

\section{THE PHENOMENA}

Dynamic pressure measurements are almost always measurements of rapid pulses. In many applications, the pressure pulse is accompanied by a dynamic temperature pulse, the fluid being measured is a combusting gas, and powerful mechanical shocks and vibrations exist in the test item. Consider as an illustration a piezoelectric transducer mounted to measure chamber pressure in a cannon, the nature of the fluid, the simultaneous mechanical shock pulses, vibrations, environmental effects, mounting conditions, and the construction of transducers commonly applied. Transducers in such an installation experience mechanical stresses, electromagnetic pulses, and temperature shocks in addition to the parameter of interest. To physically protect the transducer from the process, grease, modeling clay, or some other material is often packed into the mounting hole.

The user is generally interested not only in the amplitude of the pressure pulse but in capturing the pulse for analysis (Figure 1).

\section{TRANSDUCER}

Because the process being measured is such a strain upon the transducer, it has become a common practice to "calibrate" these devices prior to and just after a test to detect drastic performance changes which may have occurred during the measurement process.

Dynamic pressure transducers exhibit hysteresis, nonlinearity, nonrepeatability, zero shift, resonances, and sudden, catastrophic failure. Their output is affected by strain on the cables, thermal transients and shocks, mechanical vibrations, electrical effects (ground loops, lead resistances, etc.), ambient conditions, photo flash, over pressure, and warm up time.

Transducers are often specified in terms of the root mean square (RMS) of linearity to a "best straight line fit" of pressure data to transducer response based upon static pressure values, repeatability, and hysteresis. Frequency response is generally indicated by specifying the resonant frequency of the device. Other sources of potential error (see Table), are well documented by manufacturers and researchers [4]. 
Figures 2 and 3 illustrate typical temperature related effects for one type transducer. Even though these effects are relatively large compared to the accuracy of the measurement desired, the effect of transient temperatures can be much greater and is not so predictable. Figure 4 is based upon illustrations from a study of the performance of miniature pressure transducers. When used without a specially constructed thermal damper, $40 \%$ performance shifts were observed. The same study found the effects of mechanical vibrations to be as much as $25 \%$ of full scale transducer output [6].

The grease or modeling clay which protect the sensor, the orifice snubber, protective shield, diaphragm, liquid cell, and mechanical construction of the device itself all act as low pass filtering elements, guaranteeing that the signal from the transducer is not identical to the actual process pulse of interest. An accurate characterization of the relationship between the process and the senor response is required to reproduce process information from transducer signal. Not only pressure information but also frequency response and repeatability data are essential to the utilization of dynamic test results. The validity of such a characterization is a direct function of the calibration process.

\section{MEASUREMENT SUPPORT}

Whether NIST support exists or not, Army dynamic pressure transducers must be certified and their performance verified. A variety of Army systems and commercial approaches to providing this measurement support are in common use. Because direct NIST traceability does not exist, each approach depends upon indirect means and calculations for estimating system accuracy. Of course, stated accuracies can often be neither independently verified nor disproved.
Direct calibration traceability to NIST through an artifact is not necessary for a measurement to be traceable to NIST. If a particular measurement depends in a well understood manner upon other parameters which are directly NIST traceable, it is theoretically possible to determine the accuracy of the measurement of interest through an error analysis calculation. Such is the justification for the systems below being identified as calibrators rather than testers.

\section{SAMPLE SYSTEMS}

Several actual, Army calibration systems are described below. None of these devices claims to adequately simulate actual usage conditions.

Shock tubes have been in use for years to produce known pressure pulses in gas (Figure 5). The chamber on the left is pressurized behind the diaphragm, which is burst when a predetermined pressure level has been reached. A pressure wave travels down the tube, stimulating the transducer. Calculations from gas theory are a possible basis; however, pressure data is generally obtained through comparison of the transducer under test to another transducer, a calibration standard. Shock tubes yield good frequency data. Weaknesses in the method include difficulty in reproducing the same pulse at the transducer, very slow repetition rate, and a generally low maximum pressure (hundreds rather than thousands of atmospheres). Also, at high pressures, such devices are somewhat dangerous.

In - contrast to the situation in dynamic pressure, static pressure measurement services, calibration, and NIST traceability are readily available. The accuracy of such measurements is parts per million up to about 7,000 Atm for liquid pressure. The device illustrated in Figure 6 takes advantage of the 
extreme accuracy and repeatability of the dead weight piston gage to produce calibrations. A precise pressure head is generated upon the transducer under test and then the valve is thrown, rapidly releasing the pressure. Transducer response is measured for a series of pressure levels. This device is ideally suited for performing repeated tests at very well defined pressure levels, for example, before and after test range shots. Concerns include the use of constant temperature oil to simulate the exploding gas actually present under use conditions, and the assumption that the transducer under test responds in the same manner to a static pressure suddenly bled off as to a positive step pressure. Such a system has excellent repeatability.

Another design, also using a piston in cylinder, generates an impulse in the fluid by dropping an anvil on the piston (Figure 7). Force applied is calculated from anvil weight and height dropped, pressure from force divided by cross sectional area. While the pressure generated is less well known than in the previous system, a true pulse is produced.

A third liquid based design is built around a very fast acting valve (Figure 8.) The chamber is pressurized and the static pressure measured by a pressure sensor which need not be fast, but should be accurate. The valve fires, exposing the transducer to a sudden, positive pressure step. The pressure gage records the new, slightly reduced pressure to which the transducer was exposed. It should be noted that the pressure gage is never exposed to significant stresses. This design shares the weaknesses of the other liquid calibrators, also assumes symmetry of response, and has the additional problem of the fast acting valve which experiences terrific stresses and must be rebuilt after every few dozen uses. It does, however, repeatably generate very high pressure calibration steps and correlates well with other measurement methods.

A final example is the pneumatic step function generator illustrated in Figure 9. This device uses a uniquely actuated, very fast poppet valve to generate step pressure pulse on a static pressure. It has proven useful for generating accurately known, repeatable performance data with either positive or negative steps. This calibrator, however, is limited to about 2,000 PSI.

\section{SUMMARY AND CONCLUSIONS}

\section{Requirements currently exist in the Army for dynamic pressure} measurements and calibration. support. Future requirements will probably far exceed those of today [6]. Calibrators which are in use do not very well simulate actual use conditions and accuracies stated are based upon estimations, comparisons, repeatability, and experience, rather than direct traceability to national standards. On the other hand, commercial and custom made calibrators probably perform sufficiently well to be practical in most applications, and could be made much more accurate if transfer standards existed which could certify and characterize their performance.

A national measurement service with a solid, scientific basis is sorely needed and an associated calibration service for transducers. The national measurement system should measure dynamic pressure and dynamic temperature in gas. The system should be capable of generating a variety of pulse and step widths. There are numeous legal and practical, operational requirements for this service. Transducers which currently exist might well prove adequate as transfer artifact standards which could be used to calibrate many of the calibration systems now in use, providing real NIST traceability. 
Measurement accuracy could well be improved an order of magnitude or more without significant changes to the current hardware base in the Army with true traceability.

Development of a service for calibrating dynamic pressure transducers will not only improve Armysupport in this parameter, but provide a significant national resource for military and civilian industries.

\section{REFERENCES}

1. Miller, James R., et. al., Results of Survey to Determine Dynamic Pressure Transducer Calibration Needs, USATSG-286-2, U. S. Army Primary Standards Laboratory, Metrology Directorate, Physical Standards and Development Laboratory, Redstone Arsenal, AL, 1986.

2. AR 750-43, Maintenance of Supplies and Equipment: Army Test, Measurement, and Diagnost ic Equipment (TMDE). U.S. Army, 1990.

AR 750-25, Maintenance of Supplies and Equipment: Test, Measurement, and Diagnostic Equipment (TMDE) Calibration and Repair Support Program, U. S. Army, 1990.

TB 43-180, Calibration and Repair Requirements for the Maintenance of Army Materie I, U. S. Army, 1990.

3. MIL-STD-45662, Mi]itary Standard: Calibration System Requirements, U. S. Department of Defense, 1990.

4. Wilson, Jon (Editor), Dynamic Pressure Measurement, Endevco P/N 30096, Endevco Corporation, San Juan Capistrano, CA 1991.

Poff, Ronald, Stable Miniature Pressure Transducer Using Inorganic Bonding Construction, Instrument Society of America, Paper \#87-02550, 1987.
Endevco Technical Papers TP 268 and 279, Endevco Corp., San Juan Capistrano, CA, 1982.

5. Johnson, Sharon M., et. al., Analys is of $\mathrm{DH}$ Model 50300 Dynamic Pressure Calibrator, USATSG-D-6-90, Physical Engineering Division, Engineering Directorate, U.S. Army TMDE Support Group, Redstone Arsena T, AL, 1990. Found that miniature transducers respond to transients that full sized ones never see.

6. Willis, Martha P., Calibration Anomalies of A Miniature Dynamic Pressure Sensor, 14th Transducer Workshop, Colorado Springs, Co, June 1987.

7. Juhasz, Arpad A., et. al., A 150,000 Pounds Per Square Inch Dynamic Pressure Calibrator, Technical Report BRL-TR-2856, U. S. Army Ballistic Research Laboratory, Aberdeen Proving Ground, MD, 1987. Mentions performance and shows chart.

8. Electrical Measurement of Weapon Chamber Pressure, ITOP 3-2-810, U. S. Army Combat Systems Test Activity, U. S. Army Test and Evaluation Comrmand, Aberdes, 1985. 


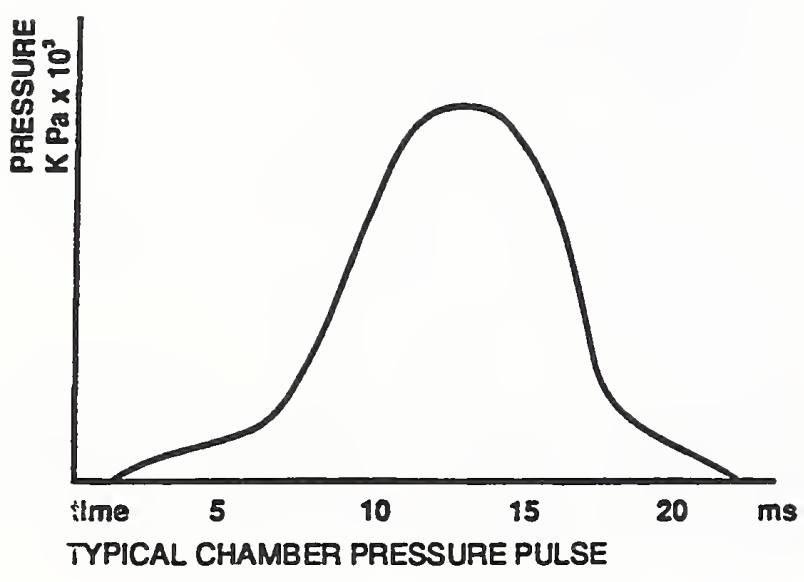

Figure 1

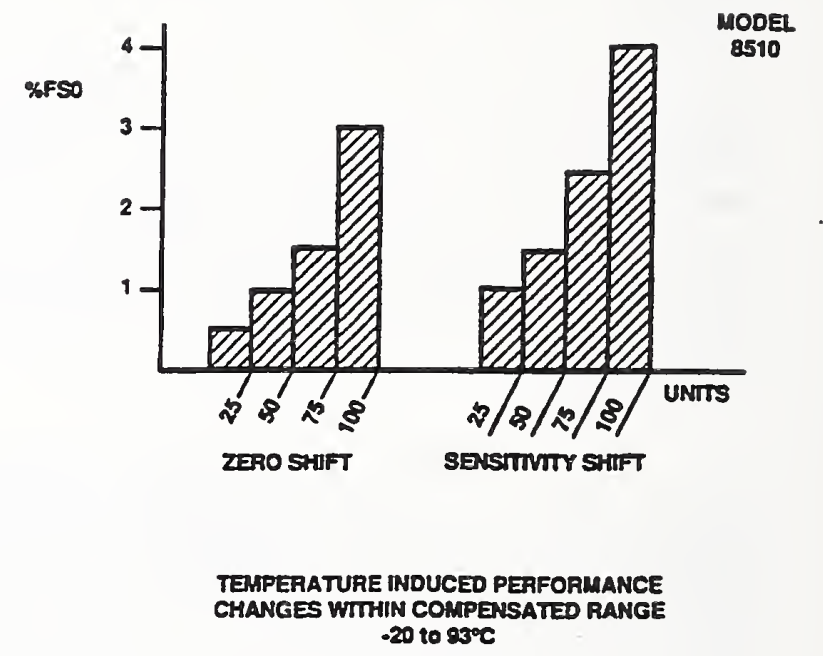

Figure 2

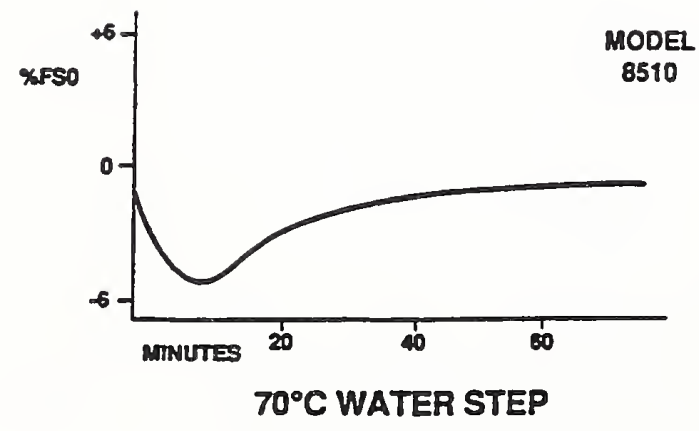

Figure 3

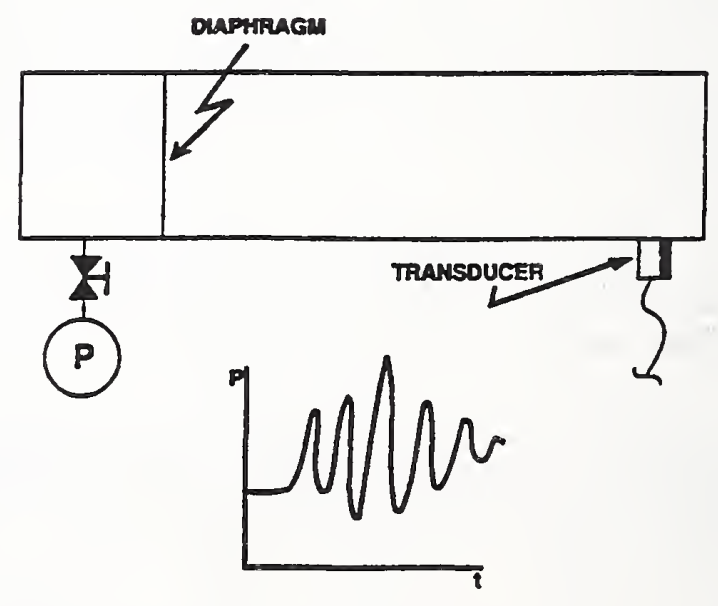

SHOCK TUBE CALIBRATOR 


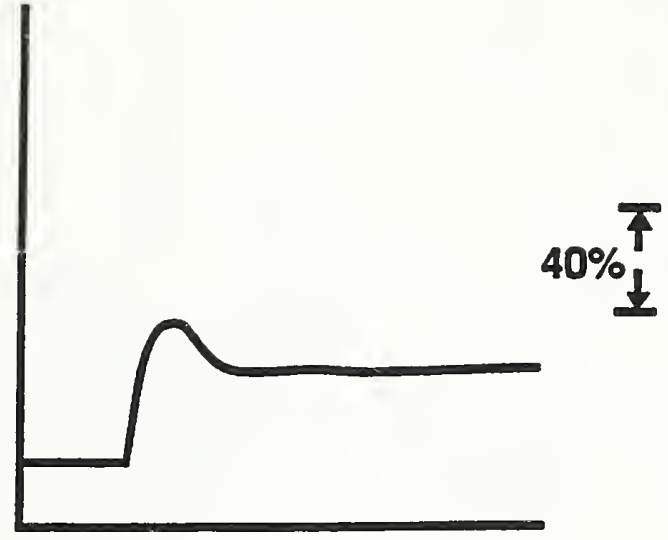

AMBIENT TEMPERATURE 100 PSI PULSE NO THERMAL BARRIER

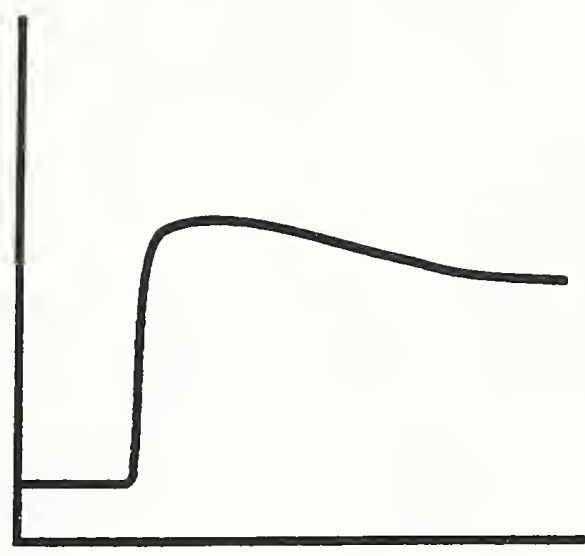

AMBIENT TEMPERATURE 100 PSI PULSE THERMAL BARRIER

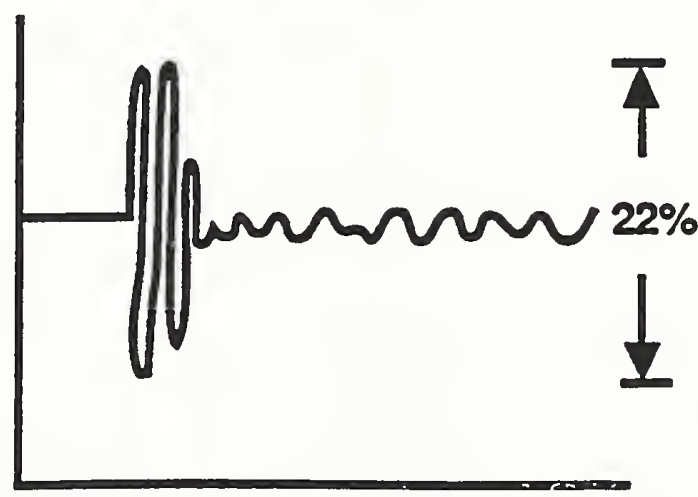

AMBIENT TEMPERATURE

NO PRESSURE PULSE MECHANICAL RESPONSE 


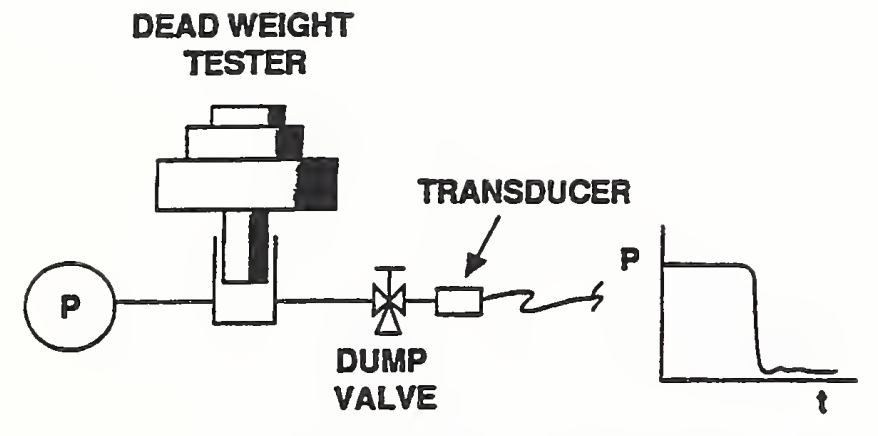

HYDRAULIC DEAD WEIGHT CALIBRATOR

Figure 6

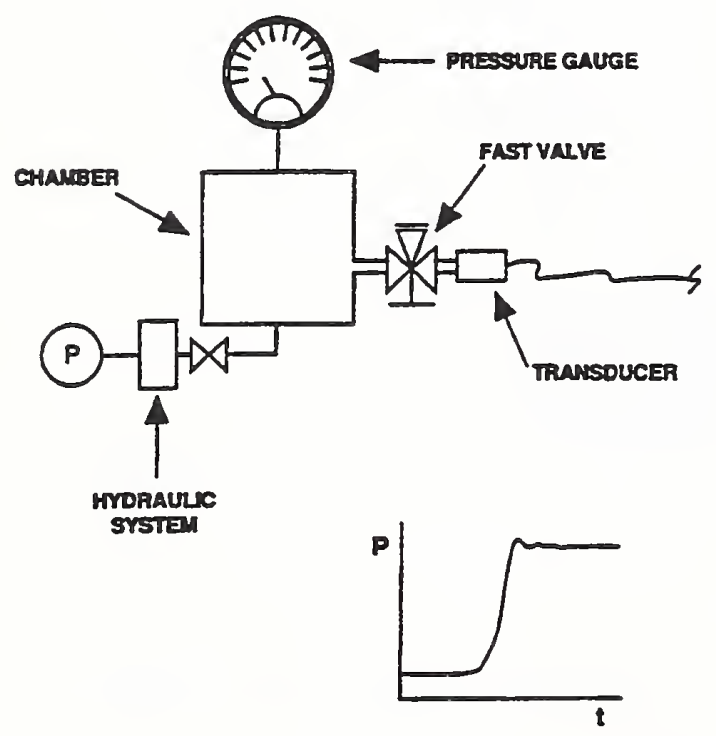

HIGH PRESSURE HYDRAULIC STEP FUNCTION CALIBRATOR
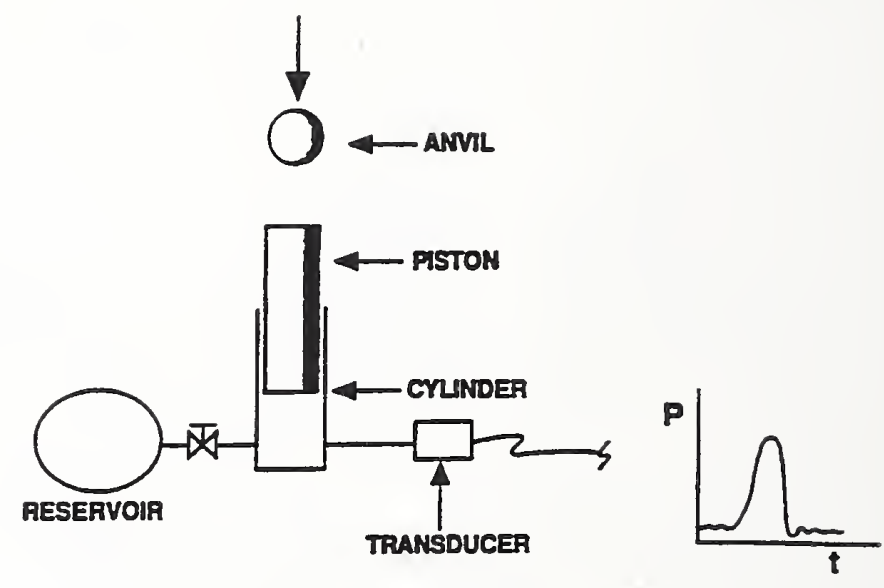

DROP BALL IMPULSE CALIBRATOR

Figure 7

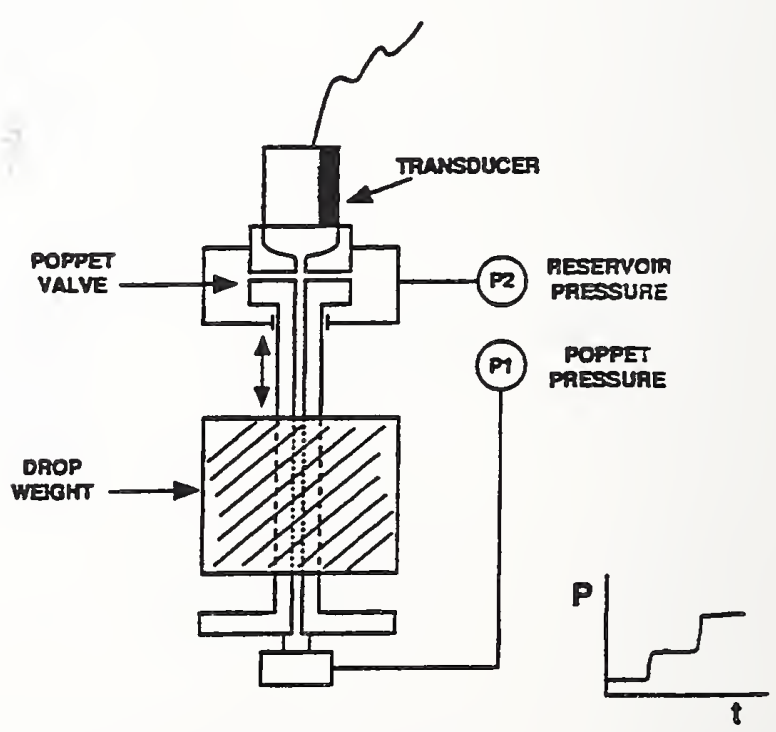

PNEUMATIC STEP GENERATOR

Figure 8

Figure 9 


\begin{tabular}{|c|c|c|c|}
\hline PARAHETER & UnTT & $\begin{array}{l}\text { TVERAEF } \\
\text { VALUE }\end{array}$ & $\begin{array}{l}\text { TIRTIIU: } \\
\text { VALUE } \\
\end{array}$ \\
\hline Full Scale Output at 10 Volts (FSO) & $\mathbf{m V}$ & 357.5 & 392.1 \\
\hline Zero Offset & $\%$ FSO & .50 & 1.40 \\
\hline Nonlinearity & $\%$ FSO & .06 & .15 \\
\hline Hysteresis & $\because$ FSO & .017 & .03 \\
\hline Nonrepeatability & $\because$ FSO & .017 & .03 \\
\hline $\begin{array}{l}\text { Combined Effect of Linearity, } \\
\text { Repeatability and Hysteresis }\end{array}$ & $\because$ FSO, RSS & .07 & .15 \\
\hline Zero Shift after $2.5 X$ Overpressure & $\because \mathrm{FSO}$ & .015 & .025 \\
\hline Input Resistance & Ohrm & 1603 & 2331 \\
\hline Output Resistance & Ohm & 738 & 1030 \\
\hline $\begin{array}{l}\text { Zero Error Due to Temperature } \\
-18 \circ \text { to } 65^{\circ} \mathrm{C} \\
-54{ }^{\circ} \text { to } 74{ }^{\circ} \mathrm{C}\end{array}$ & $\begin{array}{l}\% \text { FSO } \\
\% \text { FSO }\end{array}$ & $\begin{array}{l}.31 \\
.62\end{array}$ & $\begin{array}{r}.99 \\
1.96\end{array}$ \\
\hline $\begin{array}{l}\text { Sensitivity Error Due to Temperature } \\
-18 \circ \text { to } 65{ }^{\circ} \mathrm{C} \\
-54{ }^{\circ} \text { to } 74{ }^{\circ} \mathrm{C}\end{array}$ & $\frac{\%}{\%}$ & .25 & $\begin{array}{r}.5 \Xi \\
1.39\end{array}$ \\
\hline $\begin{array}{l}\text { Diaphragm Resonant Frequency } \\
15 \text { psia } \\
50 \text { psia } \\
100 \text { psia } \\
200 \text { psia }\end{array}$ & $\begin{array}{l}\mathrm{Hz} \\
\mathrm{Hz} \\
\mathrm{Hz} \\
\mathrm{Hz}\end{array}$ & $\begin{array}{l}140,000 \\
240,000 \\
280,000 \\
400,000\end{array}$ & \\
\hline $\begin{array}{l}\text { Flat Frequency Response of Trans- } \\
\text { ducer }\end{array}$ & $\mathrm{Hz}$ & 250 & \\
\hline Warmup Time ( $1 \%$ Accuracy) & $\mathbf{m S}$ & 1 & \\
\hline Acceleration Sensitivity & $\because \mathrm{FSO} / \mathrm{g}$ & $1.5 \times 10-4 \% / g$ & \\
\hline Zero Shift with Mounting Torque & $\because$ FSO & .1 & \\
\hline Nonlinearity at $2 \mathrm{X}$ Range & $\% 2 \times$ FSO & .2 & \\
\hline Insulation Resistance at 50 Vdc & Megohm & 1,000 & \\
\hline Noise (DC to $50,000 \mathrm{~Hz}$ ) & $\mu V \mathrm{rms}$ & 5 & \\
\hline
\end{tabular}

\section{(After Poff)}




\title{
THE SEARCH FOR A TRANSIENT PRESSURE STANDARD: A COLLABORATIVE EFFORT BY NASA AND NIST
}

\author{
Troy J. Estes \\ National Aeronautics and Space Administration \\ Johnson Space Center \\ White Sands Test Facility \\ and \\ William C. Smith, Ph.D. \\ Lockheed Engineering \& Sciences Company \\ Johnson Space Center \\ White Sands Test Facility
}

\begin{abstract}
The National Aeronautics and Space Administration (NASA) performs a variety of research requiring the measurement of transient pressures. To verify the accuracy of these measurements, NASA presently must use calibration techniques that were developed by pressure transducer manufacturers, but these techniques are unsatisfactory and fail to meet all NASA requirements.

NASA and the National Institute of Standards and Technology (NIST) metrologists have collaborated to develop an alternative calibration approach that will resolve problems with current calibration techniques and will meet critical research requirements.

This paper will discuss current calibration limitations, NASA research requirements, and the proposal for the alternative calibration approach.
\end{abstract}




\section{INTRODUCTION}

NASA research is dynamic and challenging, both in the type of research conducted and in the type of measurements made. One area that has become especially challenging is the measurement of transient pressures. The challenge is to provide accurate measurements under actual use conditions.

To ensure accuracy, NASA requires that all instruments used to gather data be calibrated and that all calibrations be traceable to national standards. NIST does not provide a transient pressure standard, so NASA presently must use calibration techniques that were developed by manufacturers.

One manufacturer's technique requires two distinct devices: a vacuum-driven shock tube and a hydraulic drop tube. The shock tube measures the timerelated responses, such as rise time. The drop tube establishes sensitivity. Between them, the pair of devices can generate pressures with amplitudes in excess of $100 \mathrm{MPa}(15,000 \mathrm{psi})$ and rise times shorter than one microsecond.

Another calibration technique, based on the Aronson calibrator, measures both time-related responses and sensitivity. This calibrator generates pressure pulses up to $7 \mathrm{MPa}$ (1000 psi) with rise times of about 35 microseconds.

Neither calibration technique is satisfying. In the first technique, the shock tube's vacuum-to-atmospheric pressure step is quite different from the atmospheric-to-pressurized step usually encountered in actual testing. Calibration with the drop tube is tedious and time-consuming, and its resolution is insufficient for the lower pressure ranges. Also, the properties of the hydraulic fluid in the drop tube are quite different from those of the gas encountered during actual measuring. The technique based on the Aronson calibrator fails to produce high enough pressures or fast enough rise times to meet all NASA requirements. Finally, neither of these techniques can measure other important factors, such as acceleration sensitivity or flash sensitivity, nor can they be used as research tools in studies of phenomena such as supersonic shock waves.

An ideal calibration system would combine the short rise times of a shock tube and the variable pressures of a drop tube, without the drawbacks of the latter.

\section{REQUIREMENTS}

NASA research requiring transient pressure measurement falls into three main categories: explosion, aerodynamic, and engine. The specific requirements of each category follow. 


\section{Explosion Research}

This research investigates blasts and associated shock phenomena, such as would be found in hydrogen and oxygen mixing explosions, ballistic propellant measurements, or liquid fuel explosions in air. Explosion research uses only piezoelectric transducers for the measurements. Typically, the transducers must measure a single, high-pressure pulse with a short rise time. A special need is separation of the thermal response from the pressure response in flash environments.

Table 1 illustrates some general requirements of explosion research.

Table 1

Explosion Research Requirements

\begin{tabular}{|l|l|l|}
\hline Parameter & Range & Accuracy \\
\hline \hline Sensitivity & $500 \mathrm{MPa}(70,000 \mathrm{psi})$ & $1 \%$ \\
\hline Resolution & $0.1 \%$ Full Scale & Not Applicable \\
\hline Rise Time & $1 \mu \mathrm{s}$ & $5 \%$ \\
\hline Resonant Frequency & $500 \mathrm{kHz}$ & $10 \%$ \\
\hline Linearity & Not Applicable & $1 \%$ \\
\hline Repeatability & Not Applicable & $1 \%$ \\
\hline $\begin{array}{l}\text { Temperature } \\
\text { Coefficient }\end{array}$ & -160 to $150^{\circ} \mathrm{C}$ & $0.05 \%$ per ${ }^{\circ} \mathrm{C}$ \\
\hline \hline
\end{tabular}

\section{Aerodynamic Research}

This research investigates the interaction of air frames with gas streams, such as would be found in studies of shock fronts, boundary layers, and turbulent zones. Aerodynamic research generally uses strain gage transducers, but piezoelectric and piezoresistive transducers are becoming more common. Typically, the transducers must measure relatively small pressures that fluctuate 
rapidly. At times, they will need to be able to measure both transient and static responses during the same test run. Transducers are usually exposed to moderate temperatures, although tests involving vehicle re-entry into the atmosphere have very high temperatures.

Table 2 shows some general requirements of aerodynamic research.

Table 2

Aerodynamic Research Requirements

\begin{tabular}{|l|l|l|}
\hline Parameter & Range & Accuracy \\
\hline \hline Sensitivity & $700 \mathrm{kPa}(100 \mathrm{psi})$ & $0.1 \%$ \\
\hline Resolution & $1 \%$ of Reading & Not Applicable \\
\hline Rise Time & $1 \mu \mathrm{s}$ & $5 \%$ \\
\hline Resonant Frequency & $1 \mathrm{MHz}$ & $0.1 \%$ \\
\hline Linearity & Not Applicable & $0.1 \%$ \\
\hline Repeatability & Not Applicable & $0.1 \%$ \\
\hline $\begin{array}{l}\text { Temperature } \\
\text { Coefficient }\end{array}$ & -170 to $1,200{ }^{\circ} \mathrm{C}$ & $0.05 \%$ per ${ }^{\circ} \mathrm{C}$ \\
\hline
\end{tabular}

\section{Engine Research}

This research investigates the performance of rocket, jet, and heat engines. These tests use strain gage, piezoelectric, and piezoresistive transducers. Typically, the transducers must measure sustained, high pressures that rapidly fluctuate. They are usually exposed to severe environments. Often the structures required to protect the transducers complicate their response, so actual use conditions require transducer cooling and temperature compensation. Another special need is determination of the phase lag introduced by the transducer in heat engine research. 
Table 3 shows some general requirements of engine research.

Table 3

Engine Research Requirements

\begin{tabular}{|l|l|l|}
\hline Parameter & Range & Accuracy \\
\hline \hline Sensitivity & $100 \mathrm{MPa}(15,000 \mathrm{psi})$ & $1 \%$ \\
\hline Resolution & $0.1 \%$ Full Scale & Not Applicable \\
\hline Rise Time & $5 \mu \mathrm{s}$ & $5 \%$ \\
\hline Resonant Frequency & $225 \mathrm{kHz}$ & $1 \%$ \\
\hline Linearity & Not Applicable & $0.5 \%$ \\
\hline Repeatability & Not Applicable & $0.25 \%$ \\
\hline $\begin{array}{l}\text { Temperature } \\
\text { Coefficient }\end{array}$ & -50 to $300{ }^{\circ} \mathrm{C}$ & $0.02 \%$ per ${ }^{\circ} \mathrm{C}$ \\
\hline
\end{tabular}

\section{Additional Requirements}

In addition to the requirements listed in the tables, ringing and acceleration sensitivity are also important factors when measuring the responses of a transducer.

\section{PROPOSAL}

NASA and NIST metrologists collaborated to develop a joint proposal that addresses the critical NASA requirements. The objective, approach, and benefits of the proposal follow. 


\section{Objective}

The objective is to develop a calibration system for characterizing transient pressure instruments.

The calibration system will include a gas-driven shock tube capable of producing shock pressures up to $100 \mathrm{MPa}(15,000 \mathrm{psi})$ and spectroscopic instrumentation to determine the pressure using inherent properties of the gas in the tube. It will be designed so that it can be maintained by calibration laboratories and compared to the calibration system maintained by NIST.

\section{Approach}

The collaborative development will be conducted in three phases.

The first phase will be the design and fabrication of the calibration systems. Both NASA and NIST will construct calibration systems for future crosscorrelation.

The second phase will be the testing and evaluation of the performance of the calibration systems over their full pressure ranges. Accuracy limits will be determined.

The third phase will be the cross-correlation of the calibration systems using calibrated transducers. The cross-correlations will verify the traceability of the calibration process.

\section{Benefits}

The most important benefit is that NIST will have established a national standard for transient pressure. NIST will provide traceable calibrations to NASA, which will maintain a reference standard for calibration of NASA instruments.

Other agencies and organizations will benefit as well. Once this research is complete, other shock tubes can be fabricated and calibrated against the NIST national standard.

An additional benefit is that the calibration system has the potential of being developed into an intrinsic standard, which would reduce the dependence on NIST for calibration of pressure transducers.

Finally, this calibration system will contribute to a better understanding of the extremely dynamic phenomena observed in wind tunnels, rocket engines, explosions, and supersonic shock waves. 
FAST PRESSURE MEASUREMENTS:

THE NEED FOR PVDF CALIBRATION STANDARDS

\author{
Bruce Hartenbaum \\ H-Tech Laboratories, Inc. \\ California Institute of Technology \\ Bradford Sturtevant \\ California Institute of Technology
}

Fast, nanosecond street measurements in solids can be made by using a variety of transducers including manganin, ytterbium, quartz, carbon, and polyvinlyidene fluoride (PVDF). In water, PVDF is the transducer of choice because of its close impedance match to water.

There are several questions, however, concerning the accuracy of PVDF pressure measurements in water at less than $1 \mathrm{~kb}$, which is our primary area of interest:

(1) What are the effects of the strength of PVDF on charge production when the transducer is placed in a fluid subjected to uniaxial strain? In the simplest case the $d_{33}$ axis is aligned with the direction of strain in the fluid, but the PVDF experiences triaxial stress and the $d_{i j}$ respond accordingly.

(2) What is the response of PVDF to an oblique plane wave? This case is similar to, but more complex than case (1).

(3) What are the causes of observed offsets? Is the offset caused by the PVDF itself or by the data recording methods?

To answer these questions we are investigating two calibration and several data reduction techniques that should be of interest to the NIST:

(1) The use of well-established electrically driven flyer plate and shock Hugoniot technology to produce a planar shock of known pressure in a water cell for calibration of PVDF transducers.

(2) The use of differential interferometry in a water cell to infer the pressure field behind a nonuniform shock, to provide an alternate measure of pressure for evaluating PVDF.

(3) Three data reduction methods: (i) numerical analysis of digital data, (ii) analog integration, and (iii) direct recording as used in the acoustics community.

It is believed that the NIST could provide a needed role by developing standards for calibration of fast nanosecond pressure transducers and by ensuring the accuracy and the equivalence of shock Hugoniot and acoustic reciprocity methods. 


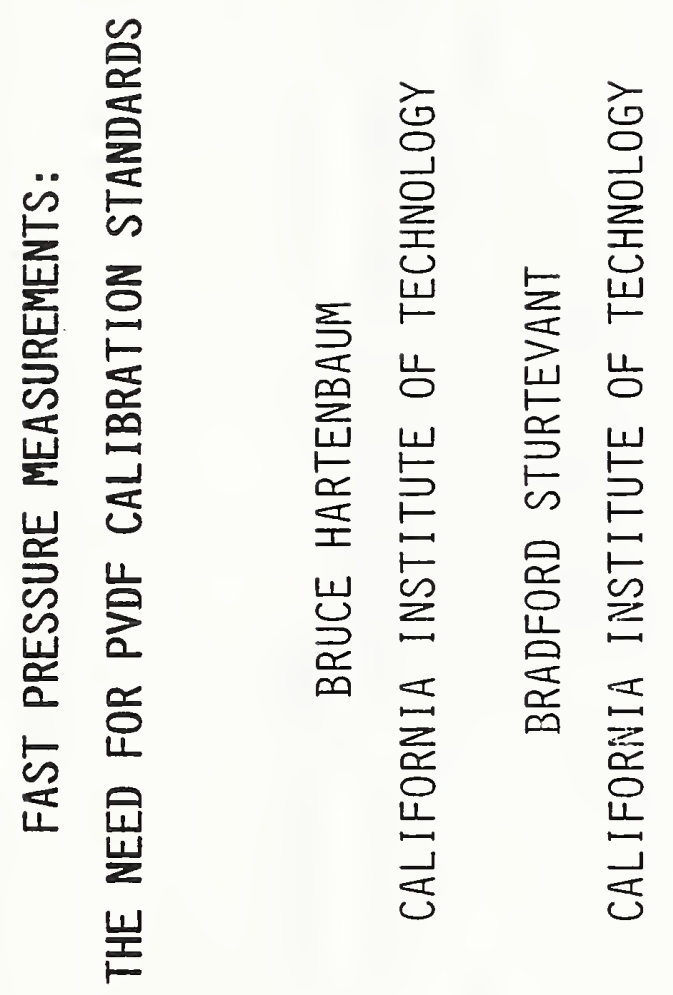




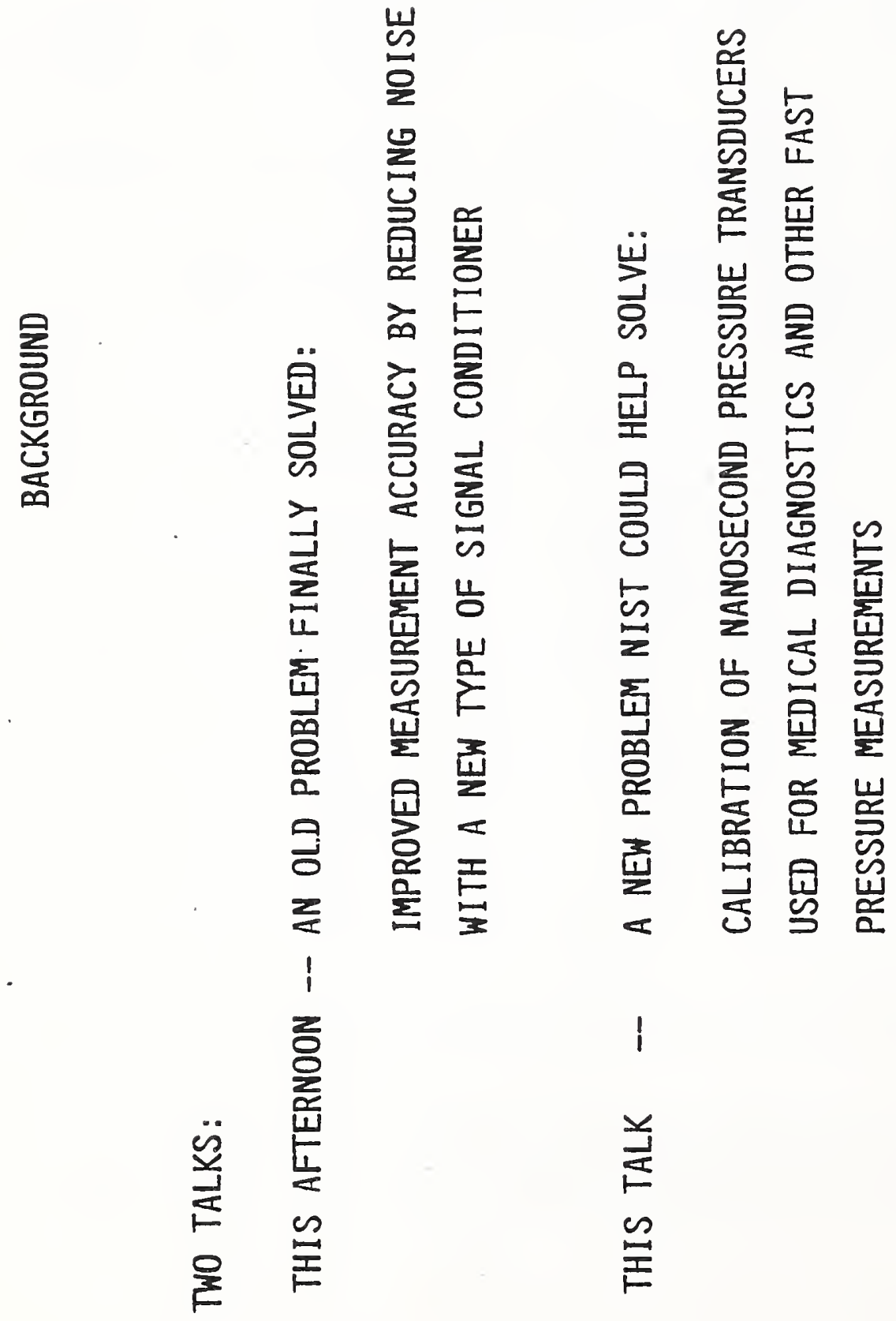




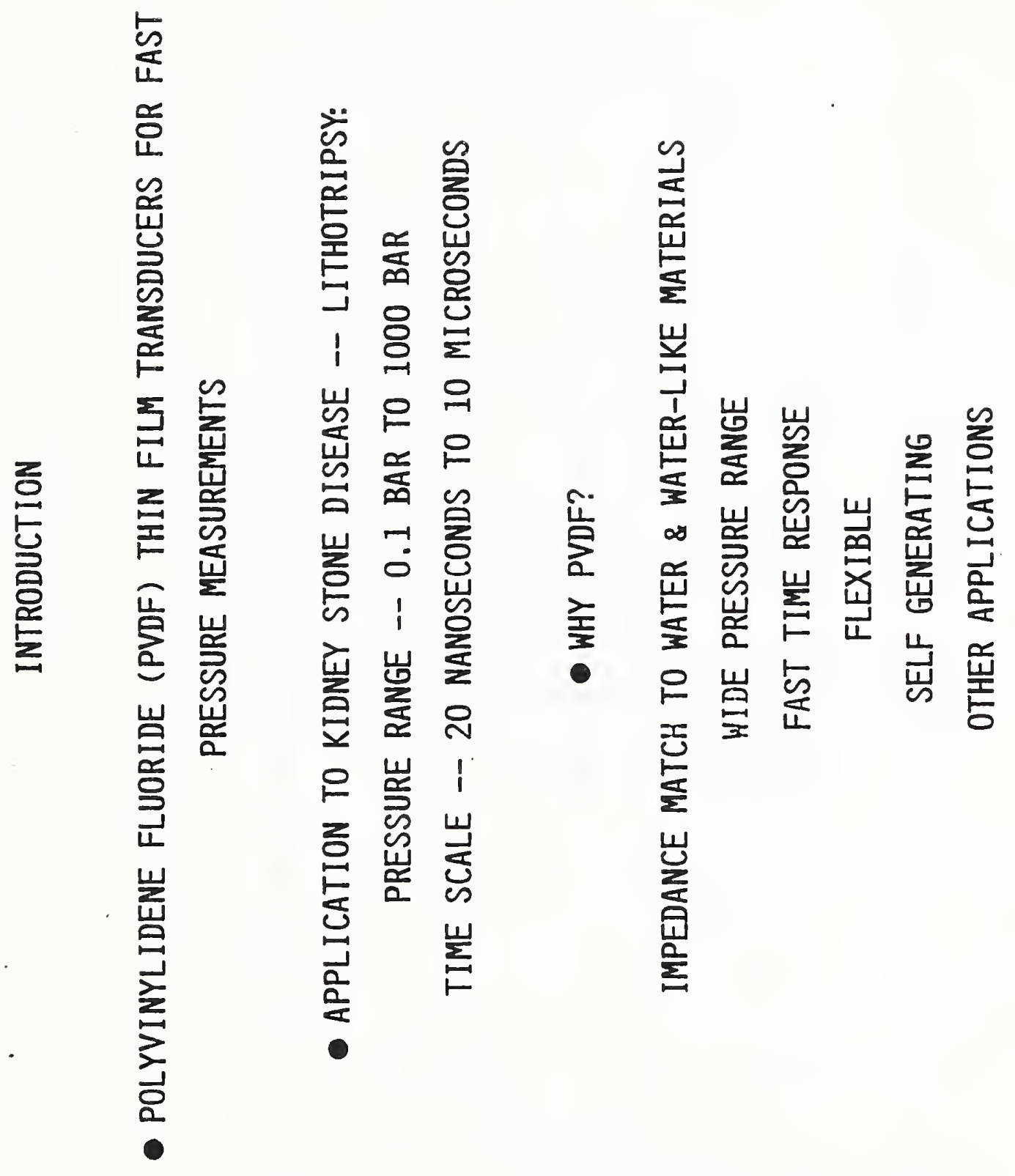


LITHOTRIPTER SHOCK WAVE PRESSURE WAVEFORMS

AT SHOCK WAVE GENERATOR EXIT PLANE \&

AT SHOCK FOCUS IN WATER BATH

(ELECTROHYDRAULIC GENERATOR)
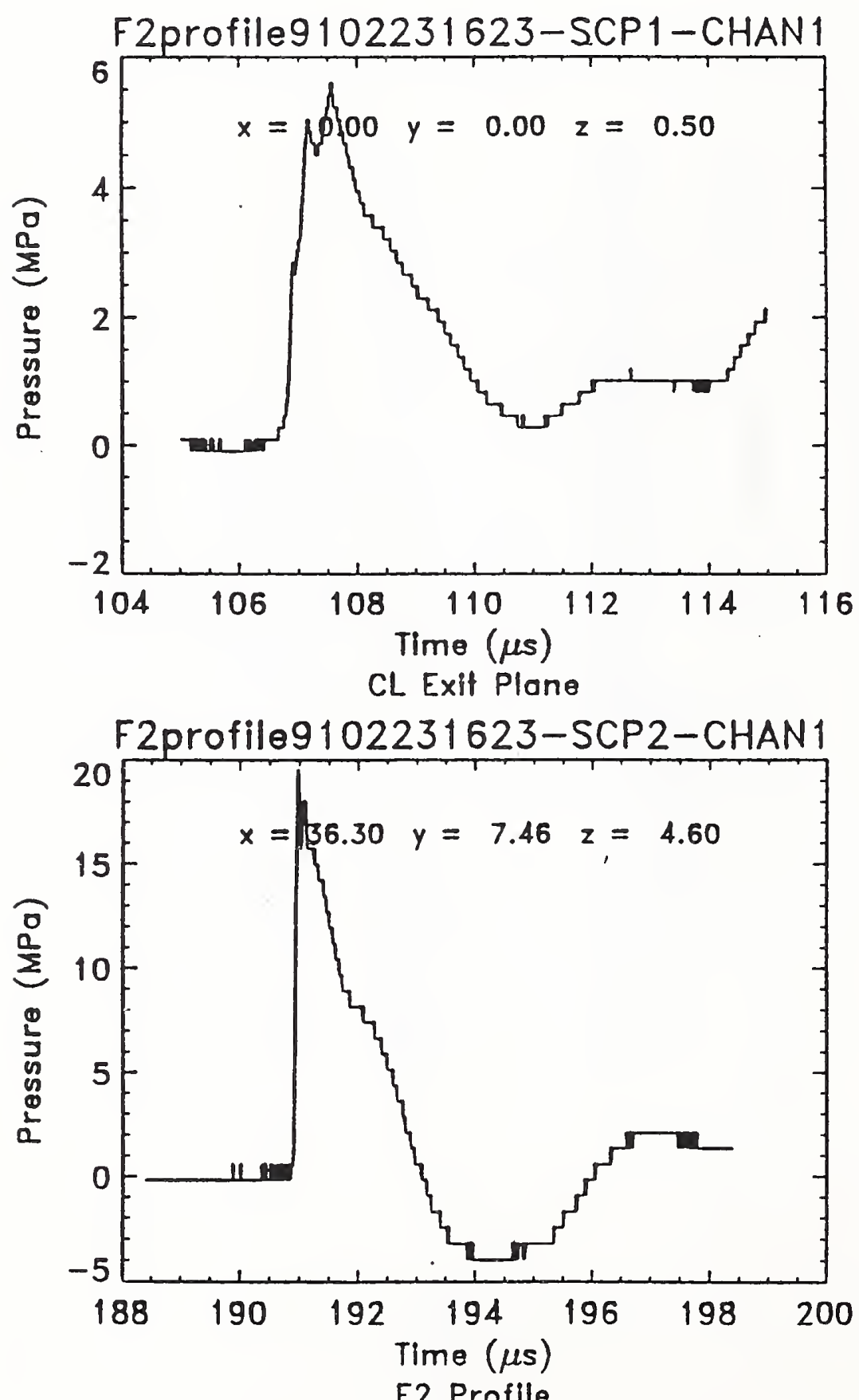


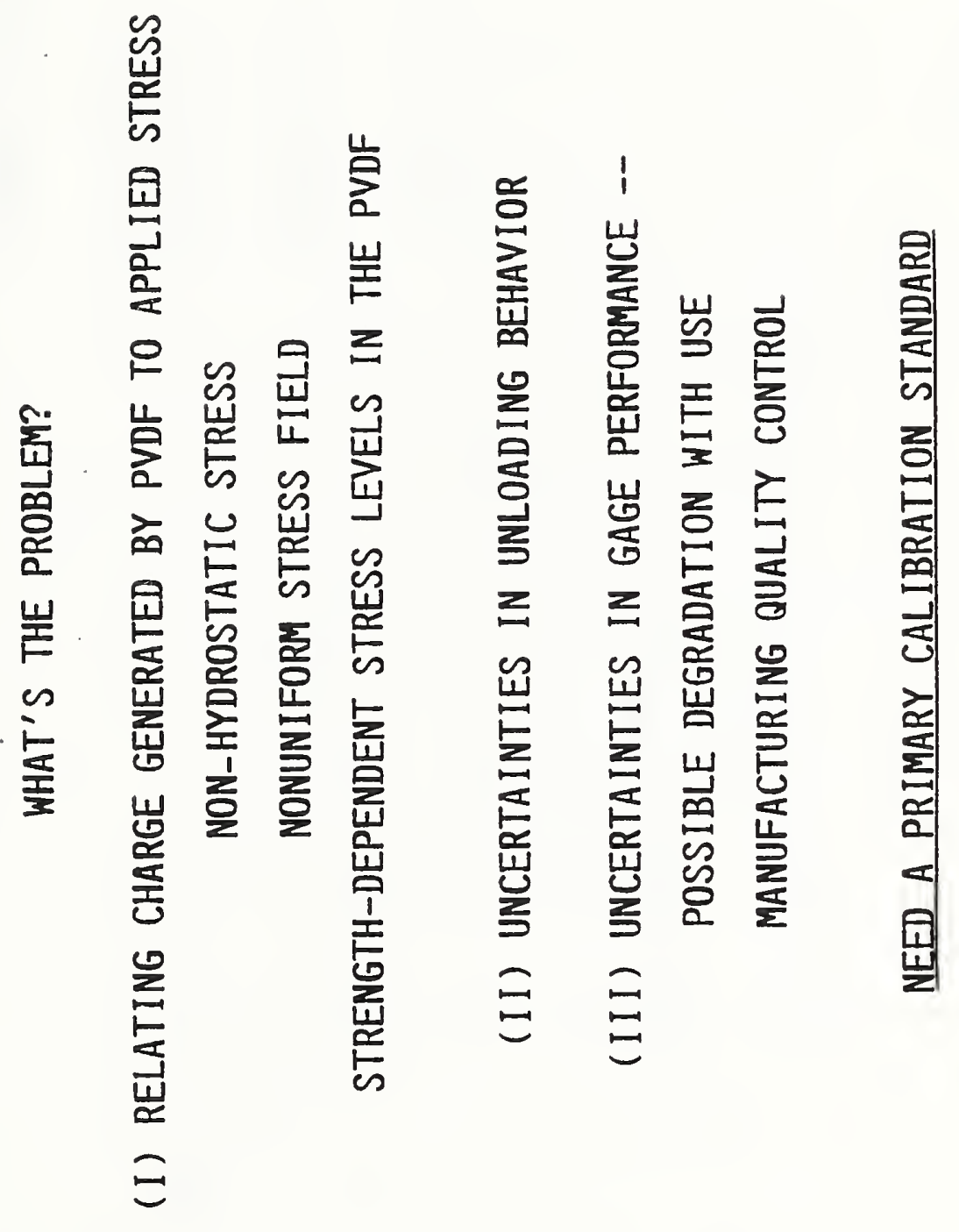




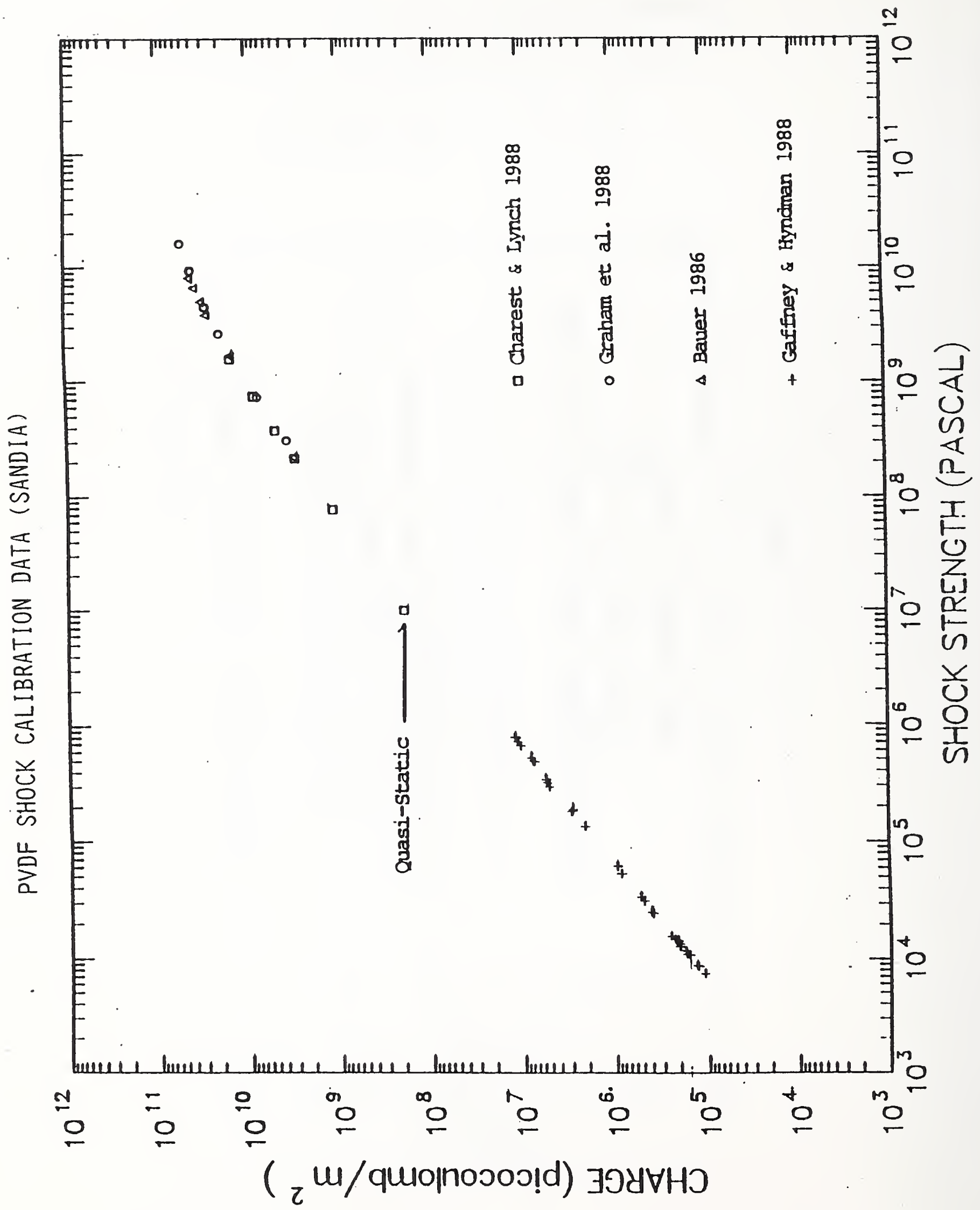



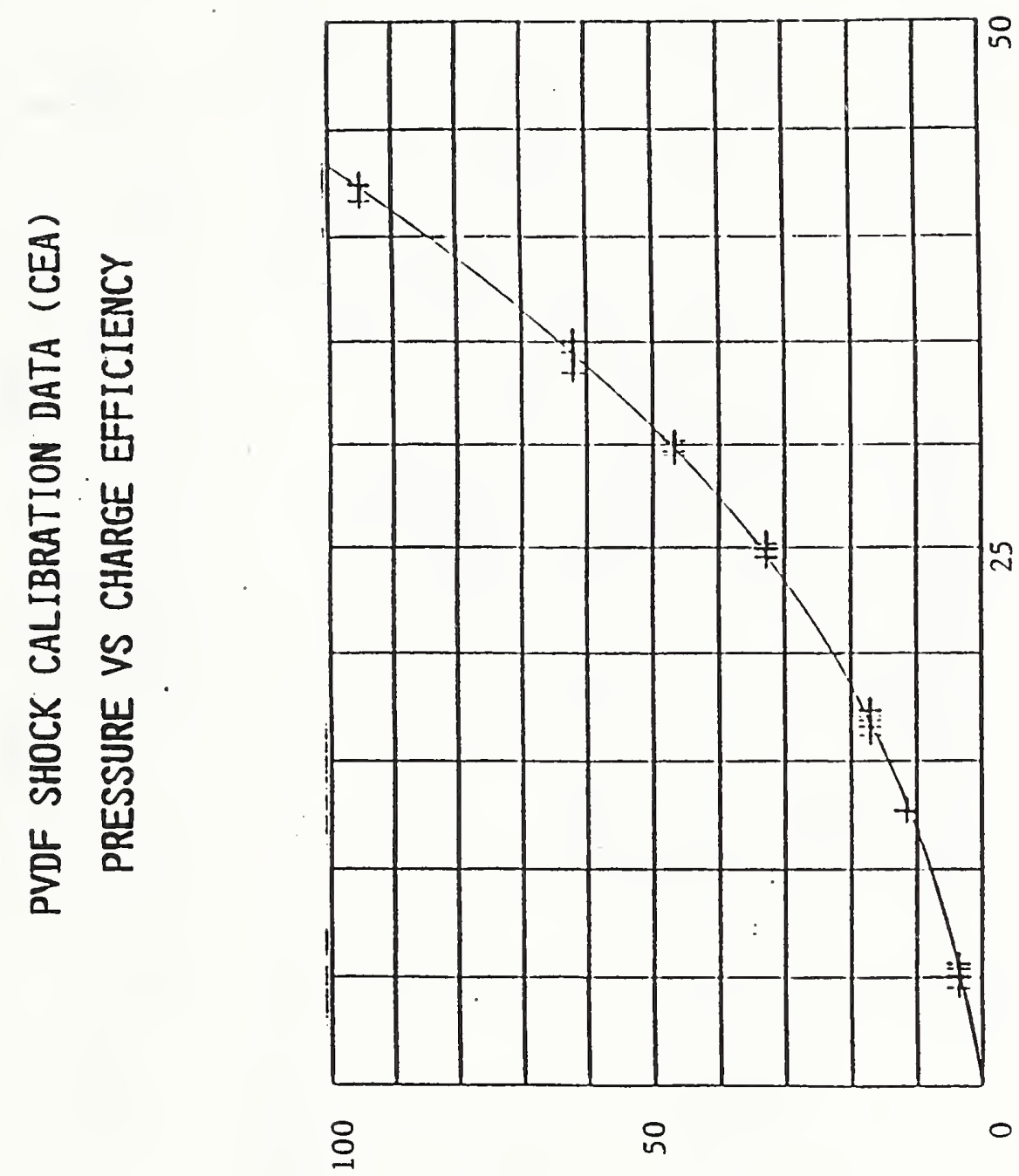

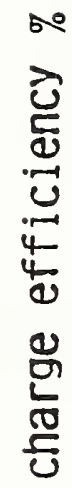

둥

$\frac{a}{5}$

ज)

느모 


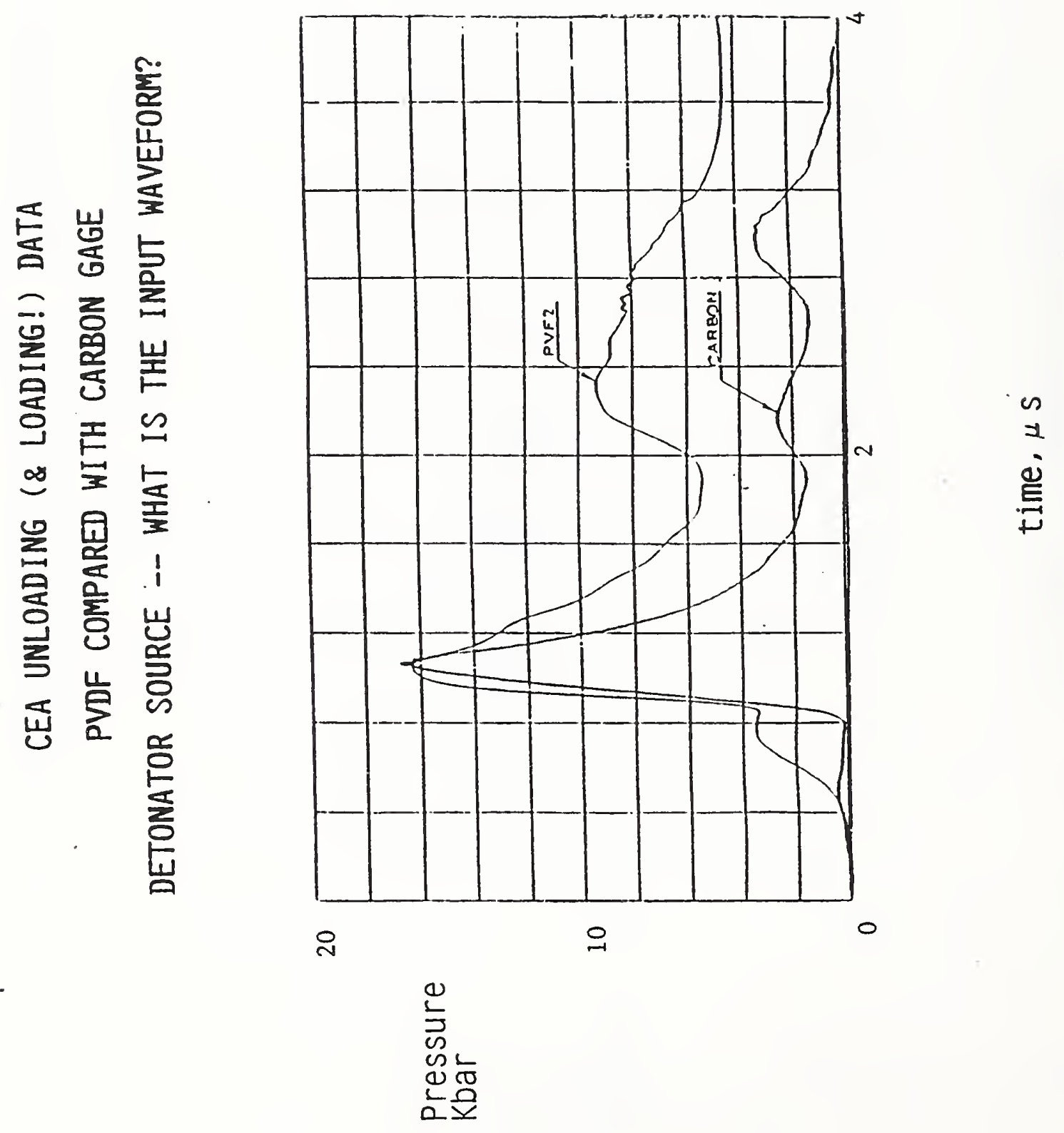



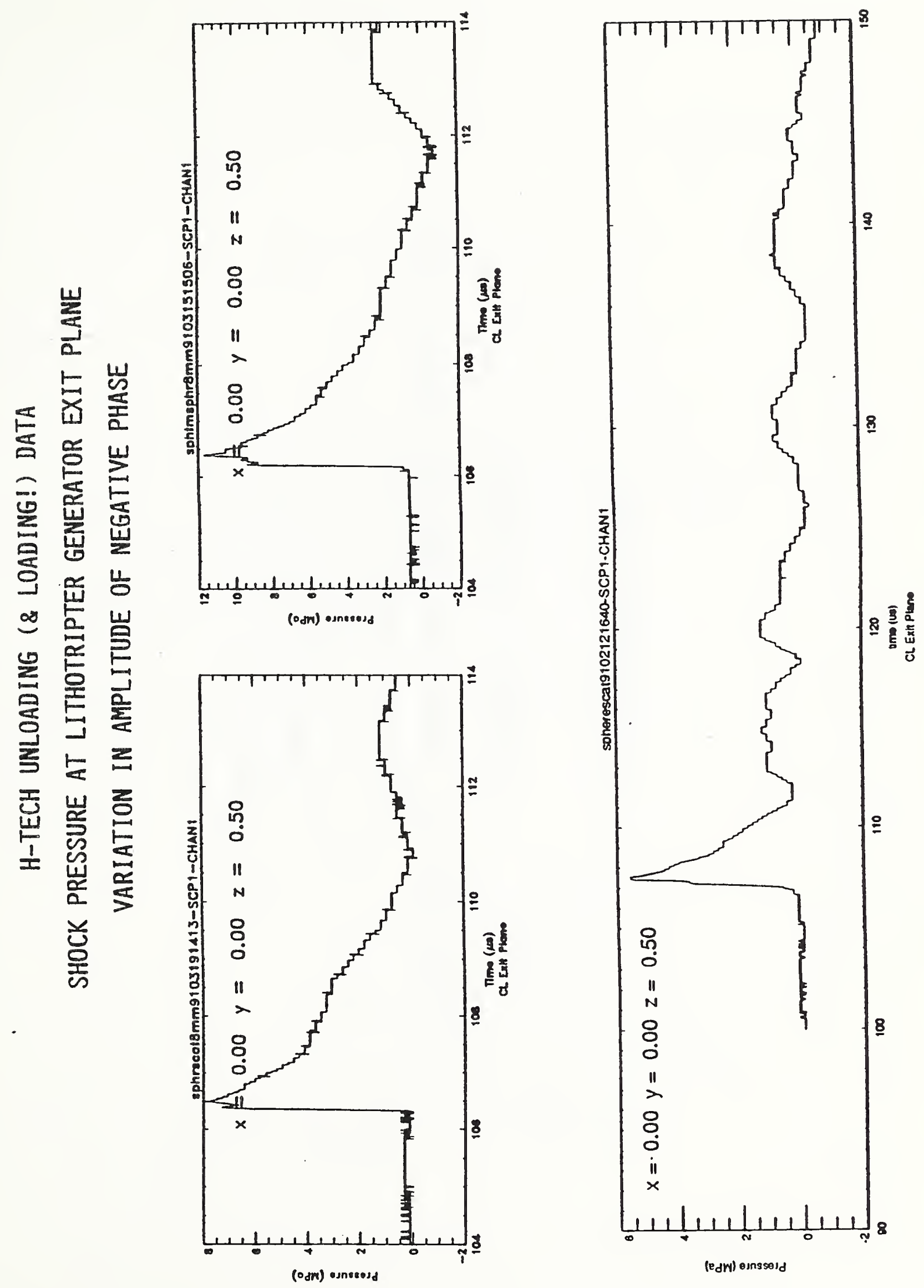


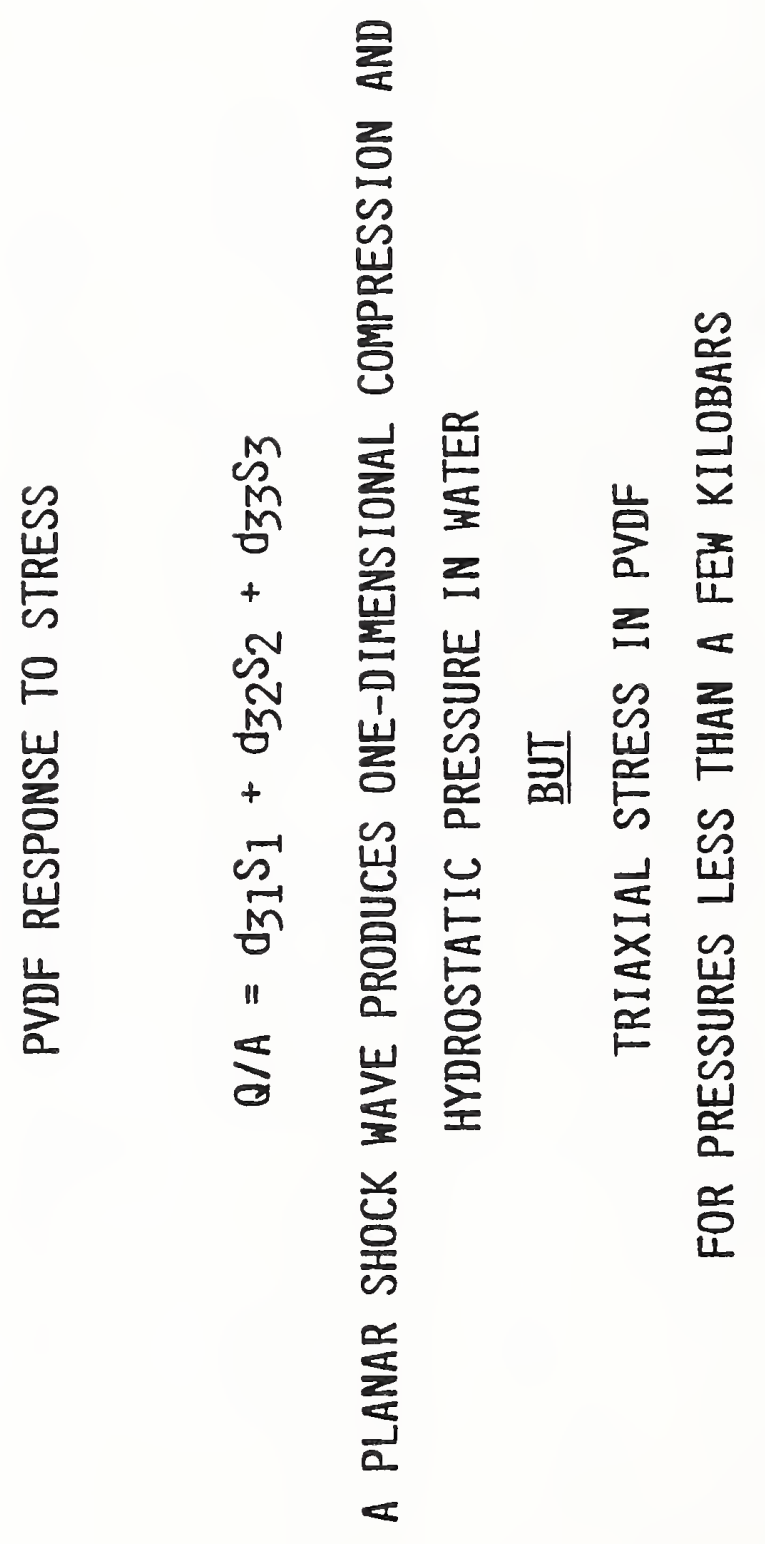




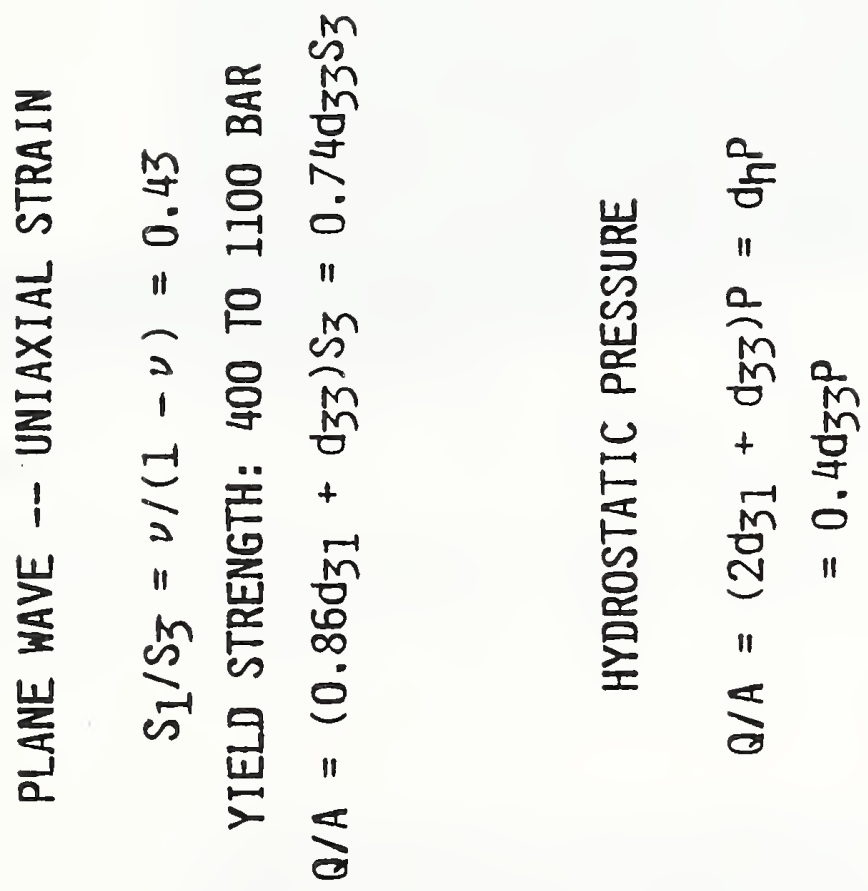




\section{TO COMPLICATE MATTERS...}

\section{NONUNIFORM PRESSURE FIELD IN LITHOTRIPTER -- NONUNIFORM LENGTH SCALE COMPARABLE TO SIZE OF TRANSDUCER}

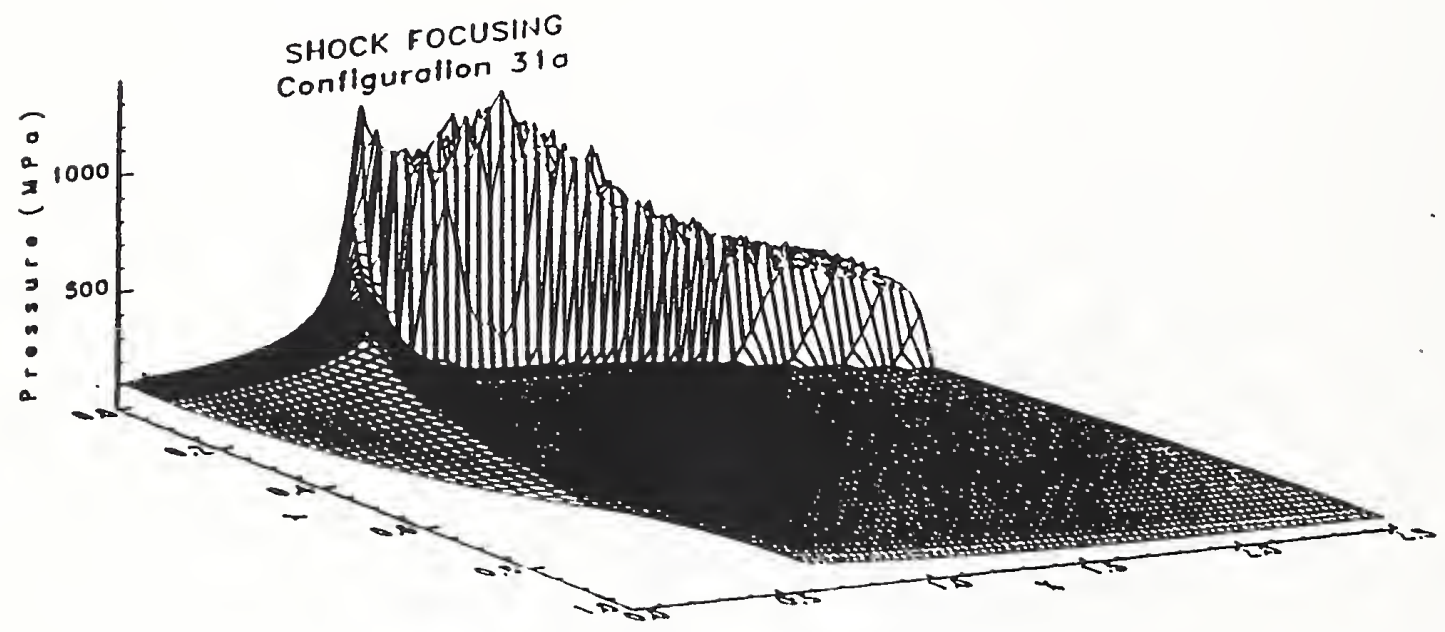

CALCULATED PRESSURE FIELD. STRONG INPUT SHOCK WITH CONSTANT STRENGTH.

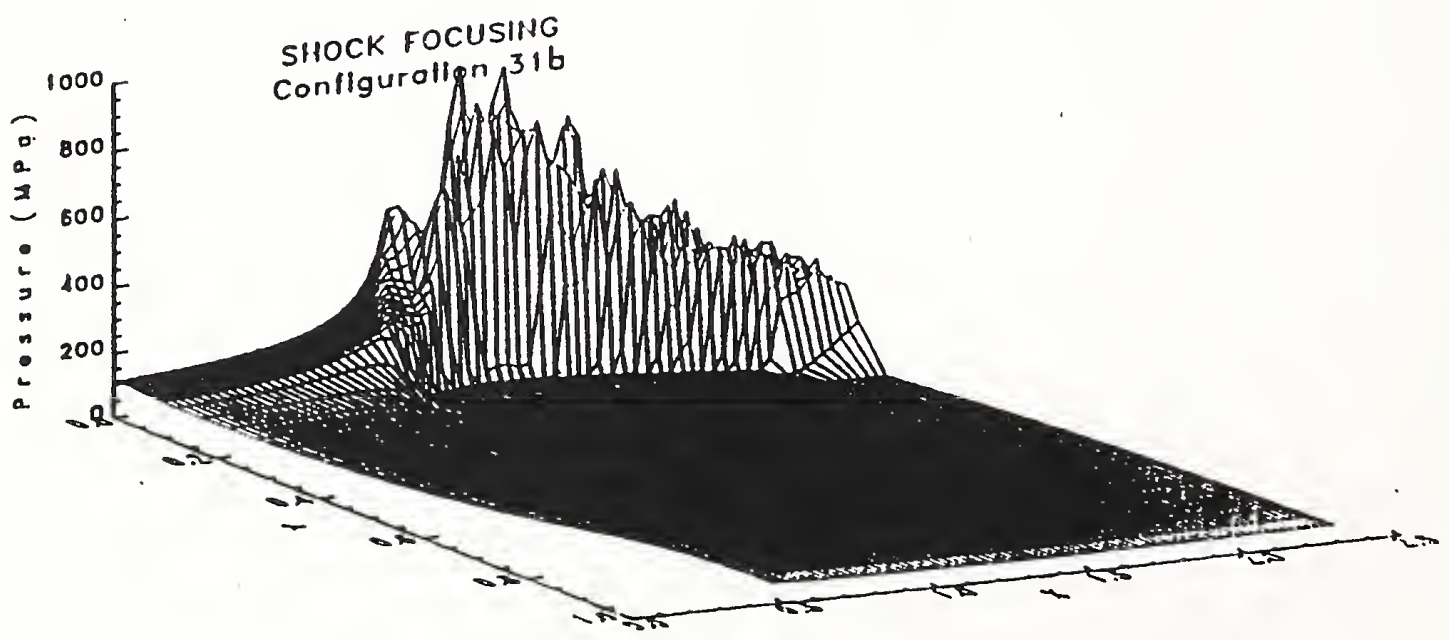

CALCULATED PRESSURE FIELD. STRONG INPUT SHOCK WITH VARIABLE STRENGTH. 
TO FURTIIER COMPLICATE MATTERS . . .

ACCURACY OF PEAK PRESSURE MEASUREMENT DEPENDS ON SIGNAL CONDITIONING

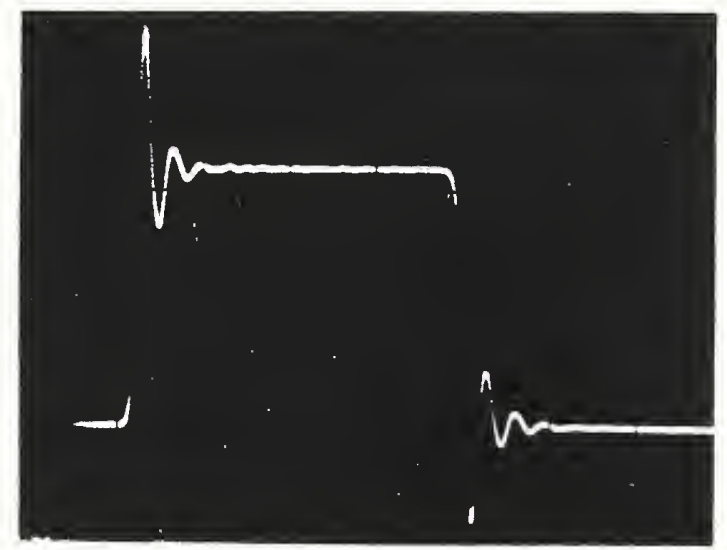

STEP RESPONSE OF MODEL OF CIRCUIT COMMONLY REPORTED IN LITERATURE (65 CM CABLE, 1 MEGOHM INPUT IMPEDANCE) a 50 NS/DIV

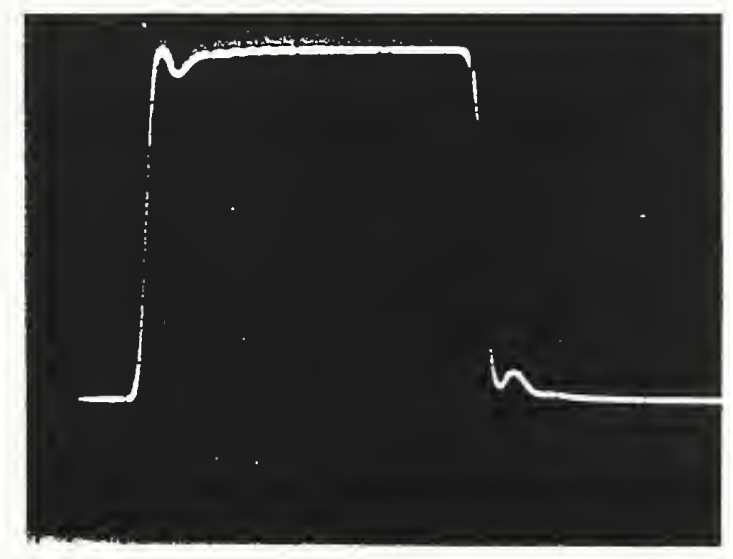

STEP RESPONSE OF H-TECH COMPENSATED CIRCUIT a 50 NS/DIV 

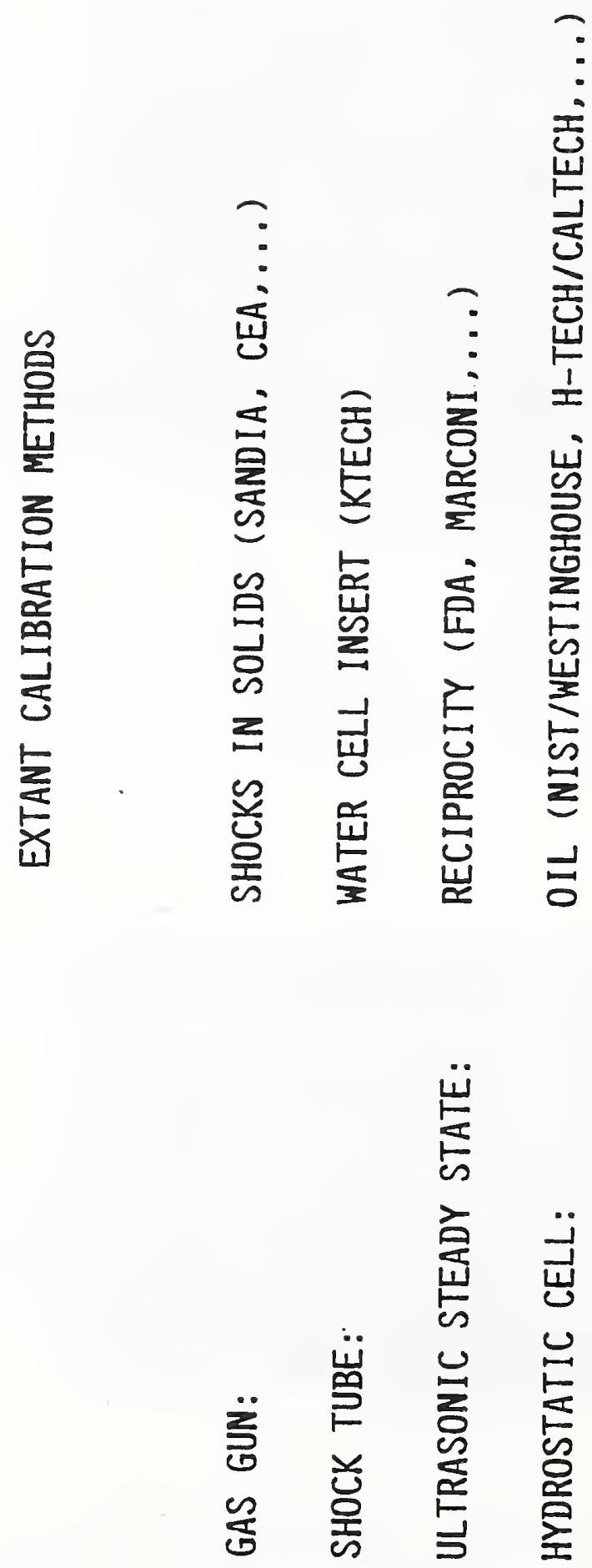

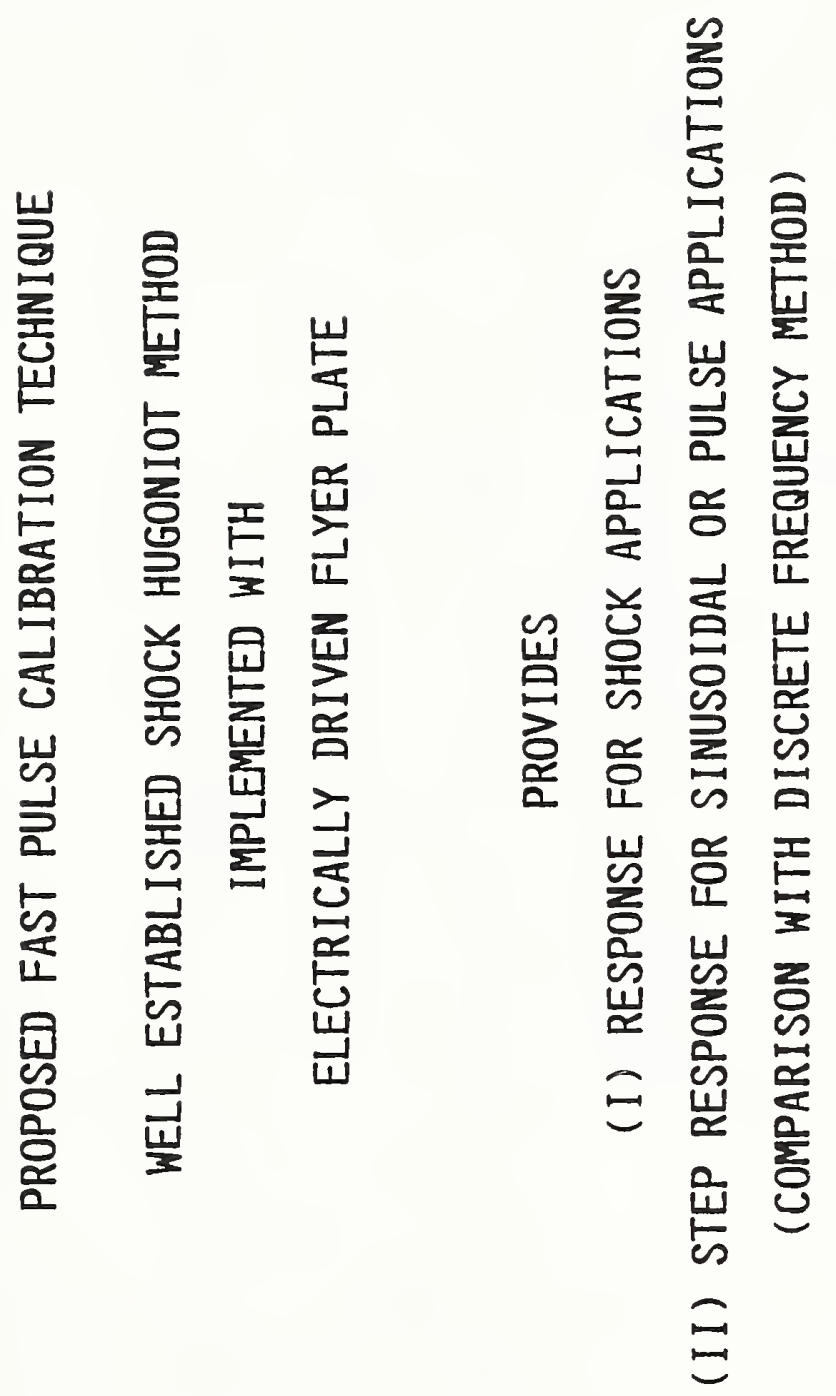


\section{SHOCK HUGONIOT CALIBRATION OF PVDF IN WATER}

SHOCK STATE IN WATER:

$$
P_{W}=\rho_{W} C_{W} U_{D W}
$$

SHOCK STATE IN FLYER PLATE AFTER IMPACTING WATER:

$$
P_{f}=\rho_{f} C_{f}\left(U_{f}-U_{p W}\right)
$$

UNIFORM PRESSURE CONDITION: $P_{W}=P_{f}$

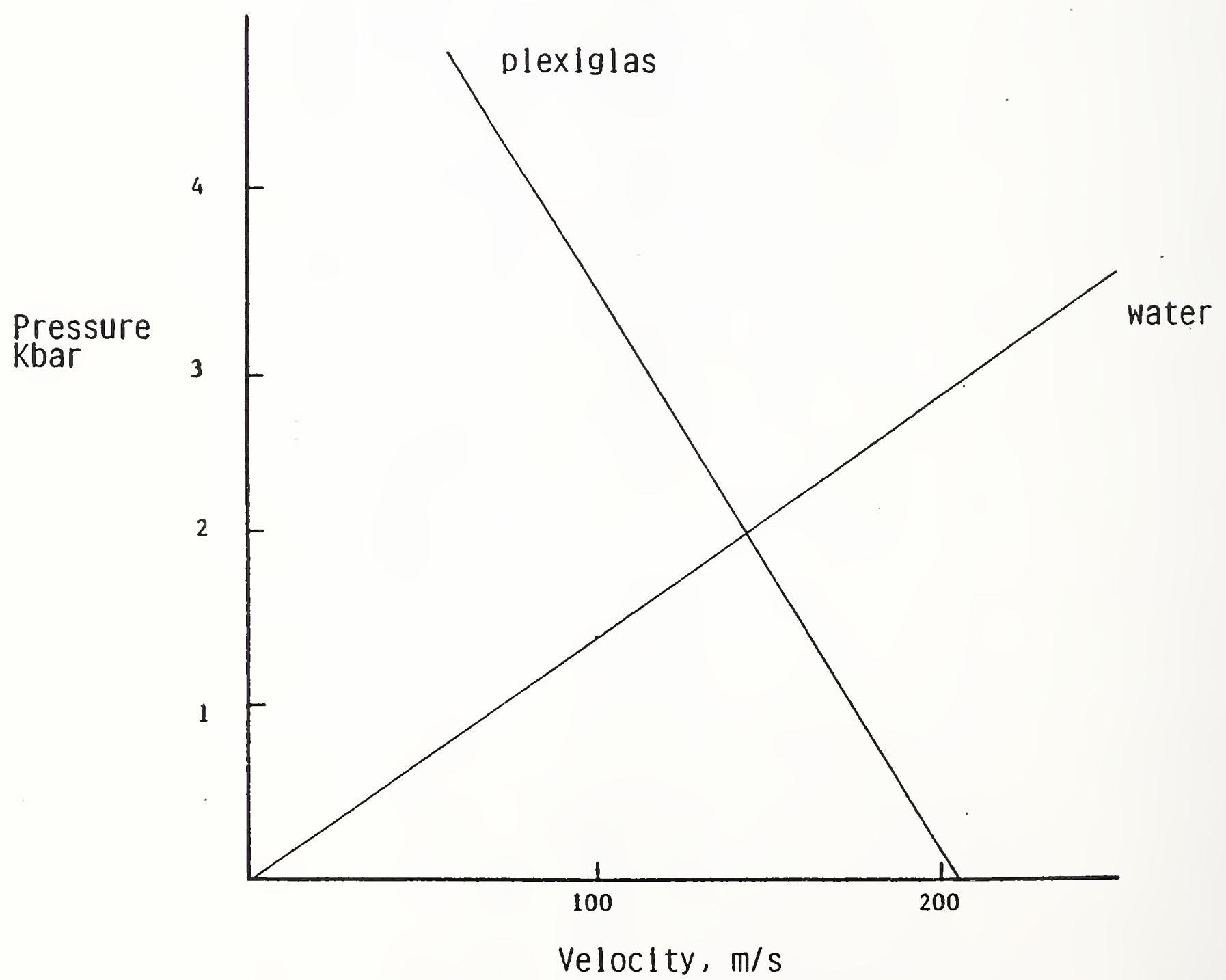



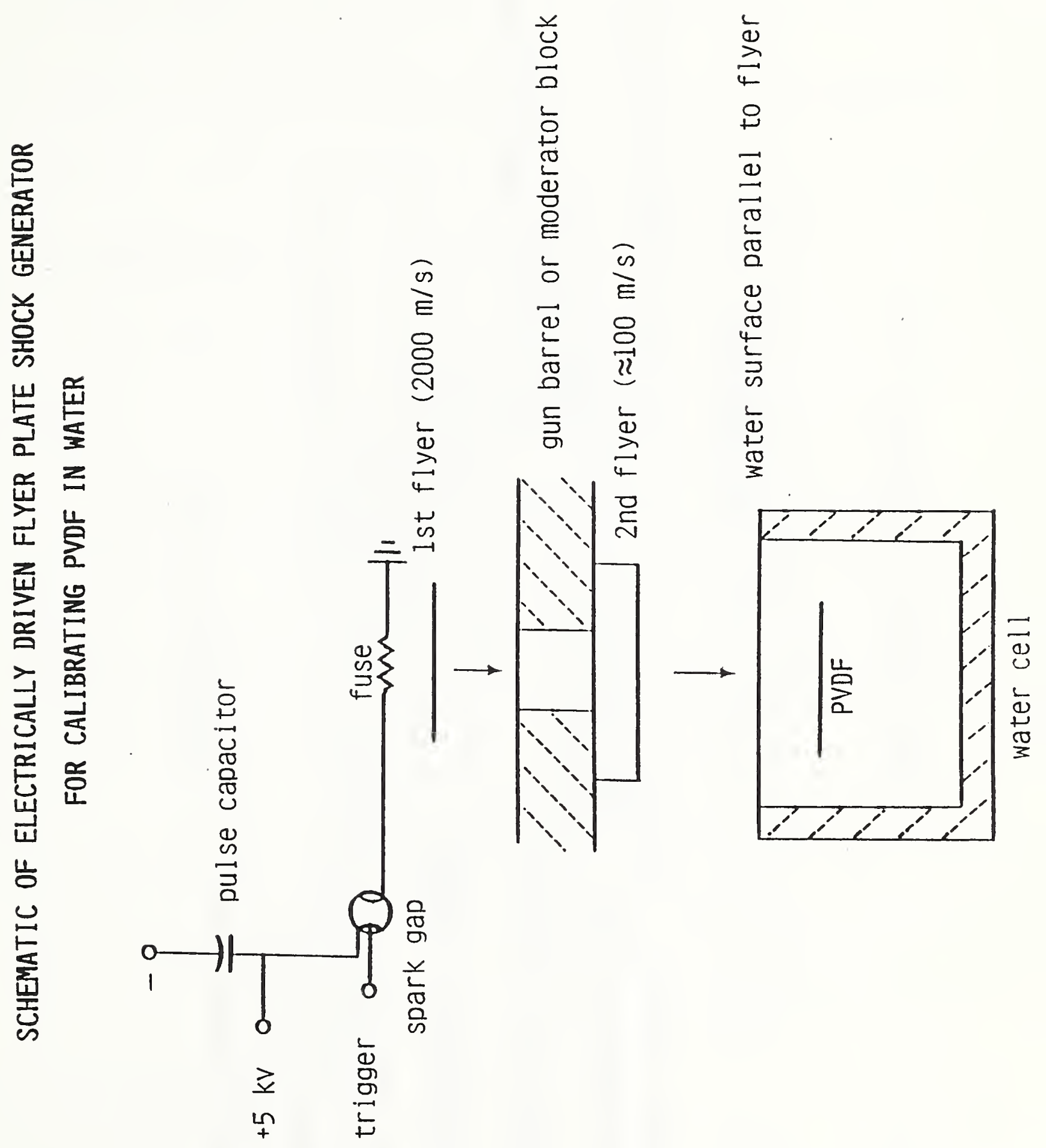


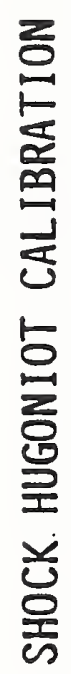

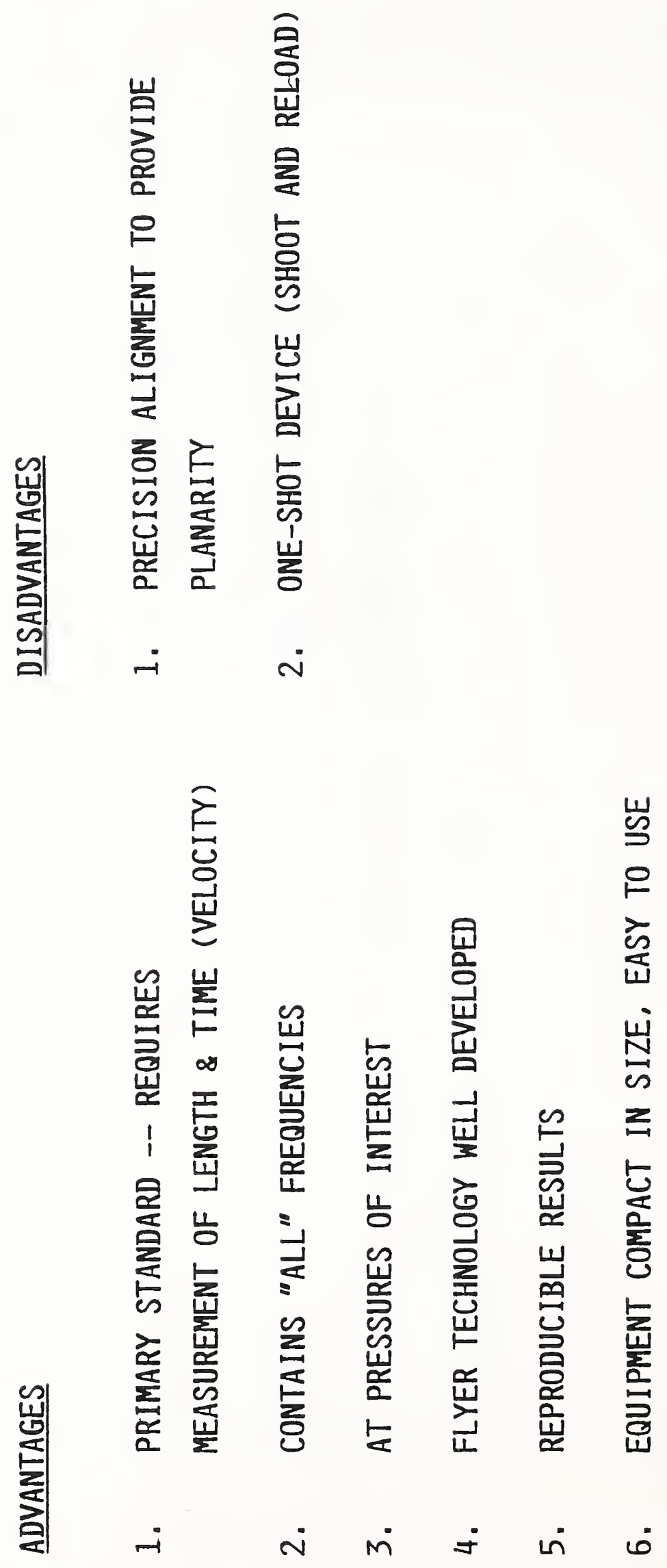




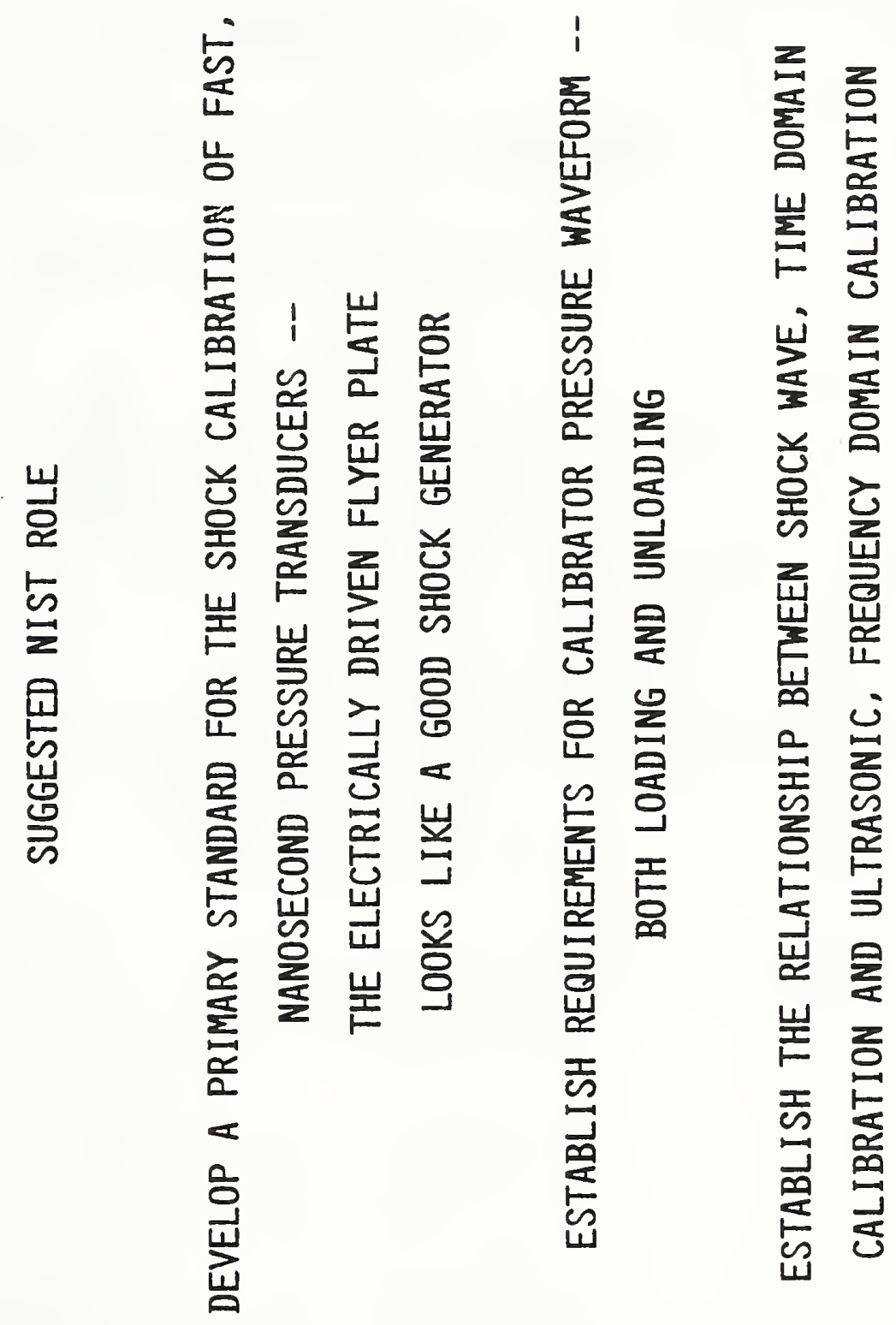




\section{DYNAMIC \\ STEP-PRESSURE \\ CALIBRATION}

Presented at: NIST (NBS) Workshop, Gaithersburg, Md. MEASUREMENT OF TRANSIENT PRESSURE AND TEMPERATURE 23 - 24 April 1991

By: J.F. Lally, President PCB Piezotronics, Inc. 3425 Walden Avenue Depew, NY 14043 


\section{New "ULTIMA" \\ DYNAMIC PRESSURE CALIBRATOR}

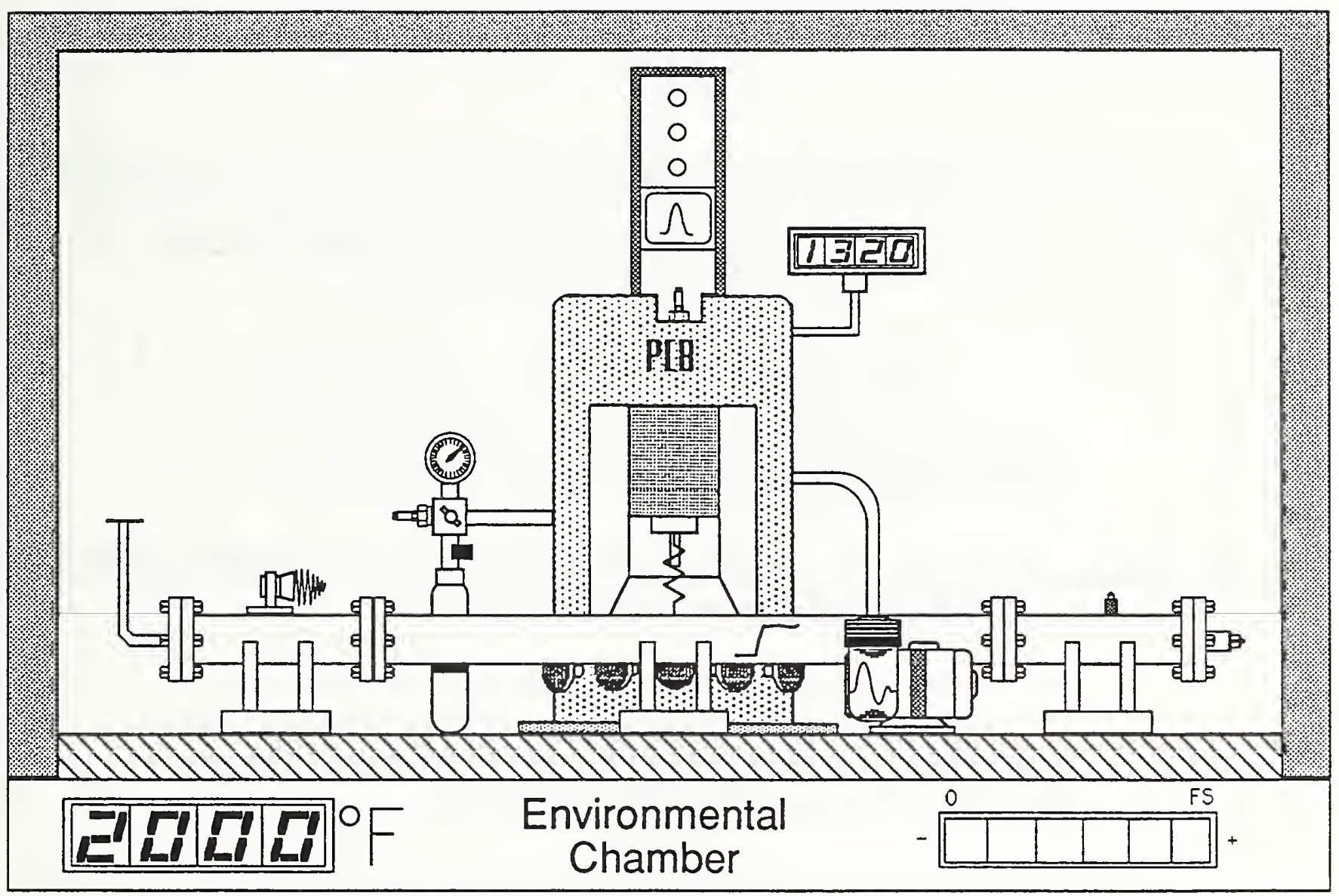

PROVIDES A CONTINUOUSLY ADJUSTABLE STEP, PULSE OR SINE WAVE PRESSURE OF KNOWN AMPLITUDE FROM:

$0.00001 \mathrm{psi}$ to $500000 \mathrm{psi}$

$\mathrm{DC}$ to $10 \mathrm{MHz}$

Absolute 0 to $+2000^{\circ} \mathrm{F}$

- COMPUTER CONTROLLED

- AUTOMATIC, SELF-COMPENSATING

- COMPLETE WITH ALL BELLS \& WHISTLES

- ECONOMICALLY PRICED AT $\$ 99.95$

- DELIVERY FROM STOCK

- NIST TRACEABLE

Manufactured by

"VISIONARY" Calibrator Co.

UTOPIA, USA 
Obviously, it would be nice if the "ULTIMA" dynamic calibration device existed. Even if it did exist, I'm not sure how it could be "calibrated". If the "ULTIMA" calibrator were dismantled, you would find it is made up of several individual special purpose calibration devices - each designed to dynamically calibrate pressure transducers over a limited pressure, frequency or temperature range. Each calibrator would pretty much represent the current state-of-the-art of dynamic calibration over the specific operating range of the device.

In 1972, considerable effort was expended by the American Society of Mechanical Engineers to establish "A Guide for the Dynamic Calibration of Pressure Transducers". The guide was originally published as American National Standard ANSI B88.1-1972; however, the current number is ANSI MC88.1-1972 (see page 3).

The ANSI calibration guide defines calibration as follows:

"Calibration means a test during which known values of measurand are applied to a transducer and corresponding output readings are recorded."

The ANSI document then proceeds to qualify expectations for lower accuracy and the somewhat more difficult nature of dynamic calibration;

"The degree of accuracy associated with these dynamic tests is generally lower, and the manner in which the results are used is generally less rigorous than in the conventional and more easily controllable field of static pressure calibration."

Much of the dynamic pressure calibration equipment used today has evolved out of the transducer manufacturer's necessity to test and calibrate his products, and by the customers' need for in-house capability to test and calibrate transducers more closely to the customers' actual application requirements. A wide variety of calibration devices exist, many of which are offered as commercial products. Several are described in the ANSI dynamic calibration guide:

- PISTONPHONE

- PULSE CALIBRATORS USING FAST-ACTING VALVES

- SINUSOIDAL PRESSURE GENERATORS

- IMPULSE DROP CALIBRATORS

- SHOCK TUBE

Most of the above are traceable to the NIST through an accurate DC reference gage, comparison transfer standard transducer or calculations of physical quantities of mass, gravity, velocity and temperature. 


\section{AMERICAN NATIONAL STANDARD}

\section{A Guide for the Dynamic Calibration of Pressure Transducers}

ANSI B88.1 - 1972

[Note: The current number for this standard is ANSI MC 88.1-1972]

SECRETARIAT

THE AMERICAN SOCIETY OF MECHANICAL ENGINEERS

PUBLISHED BY

THE AMERICAN SOCIETY OF MECHANICAL ENGINEERS United Engineering Center 345 East 47th Street New York, N. Y. 10017

Pim.tic

Li. 3 arm

$H(-35) 1-19-2(k: \lim 7 \pi)$ 

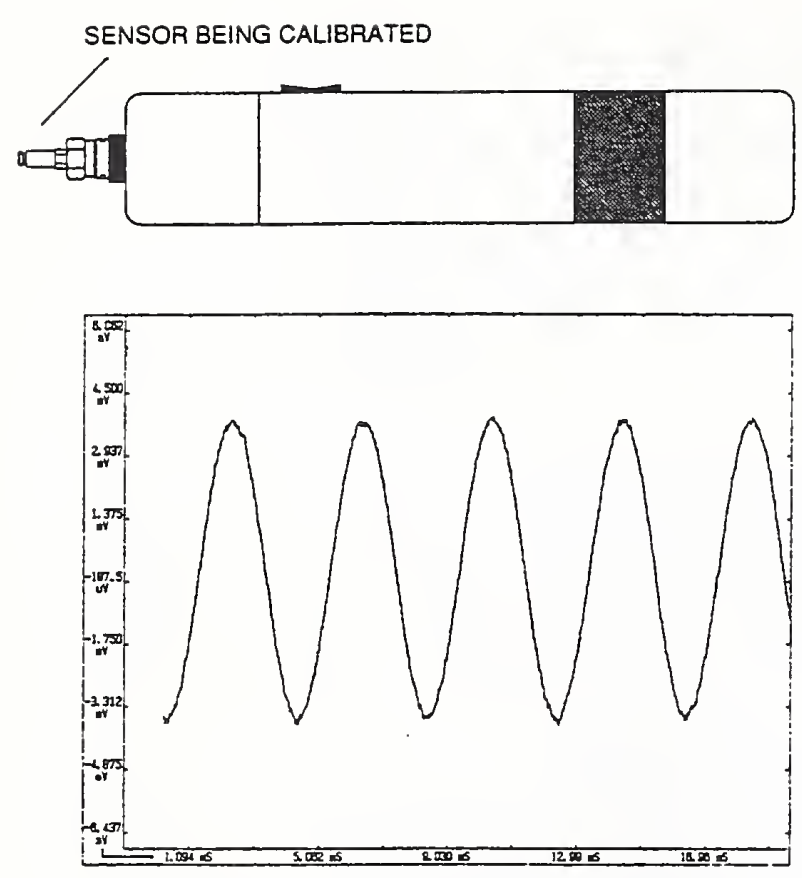

Plstonphone Output $124 \mathrm{~dB}, 250 \mathrm{~Hz}$

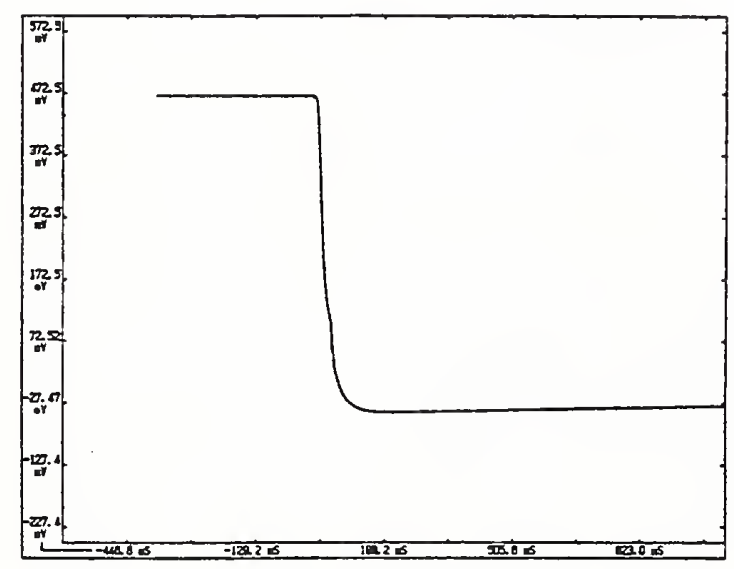

Pressure Release 100 psi Response Time $60 \mathrm{~ms}$

NIST TRACEABILITY - Dead Weights

\section{PISTONPHONE}

The pistonphone is designed to provide a precision sound reference source at a fixed frequency and amplitude for calibration of low pressure acoustic sensors. It is usually pack aged in a compact, portable battery-powered configuration. Calibration accuracy is claimed to be $\pm 0.2 \mathrm{~dB}$ with certain specified microphones.
DEAD WEIGHT TESTER USED AS A PRESSURE RELEASE CALIBRATOR

The dead weight tester is designed and most commonly used for static calibration of DC type pressure transducers such as strain gages, capacitive, piezoresistive, or quartz piezotypes exhibiting long discharge time constants. The dead weight tester can also be used dynamically in the pressure release mode for transducers whose output characteristics in response to pressure rise and pressure drop have been determined to be the same.

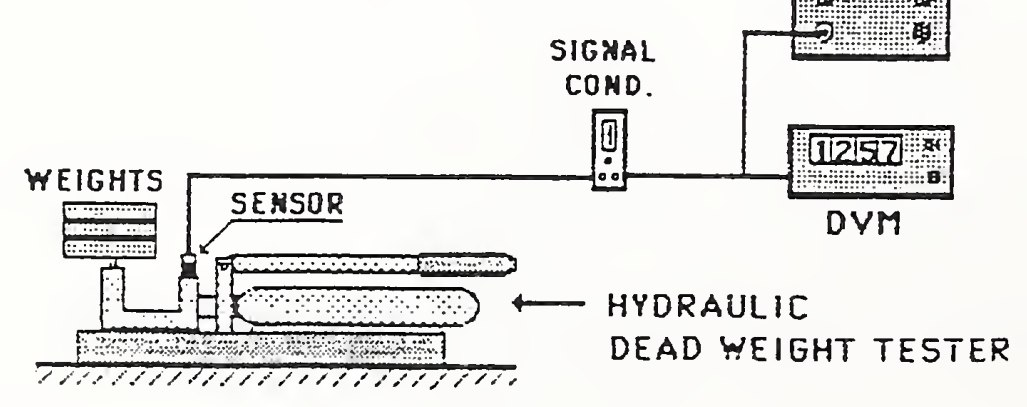




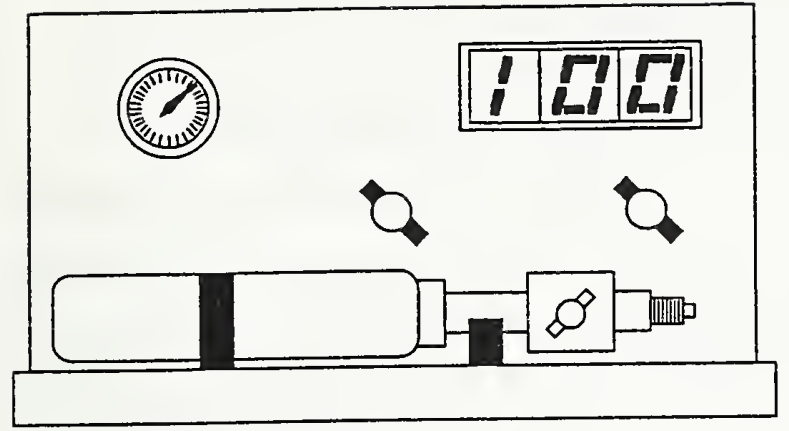

Pneumatic Pulse Calibrator PCB Model 903A

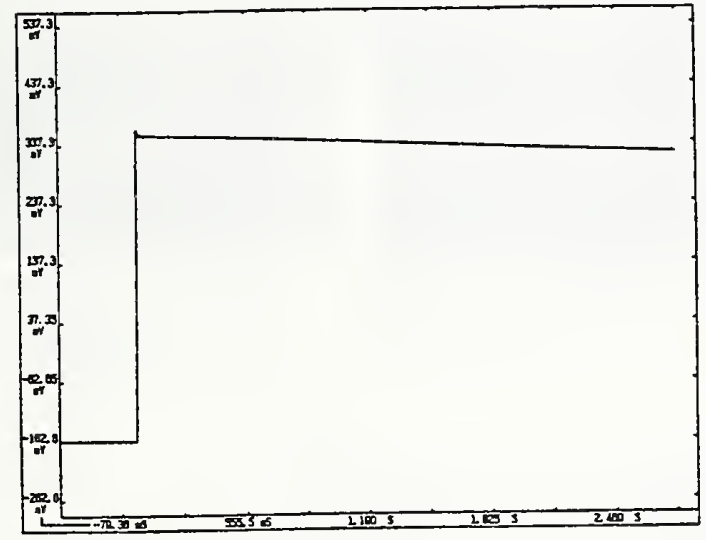

Step Pressure $100 \mathrm{psi}$

Rise Time $5 \mathrm{~ms}$

NIST TRACEABILITY - DC Reference Gage

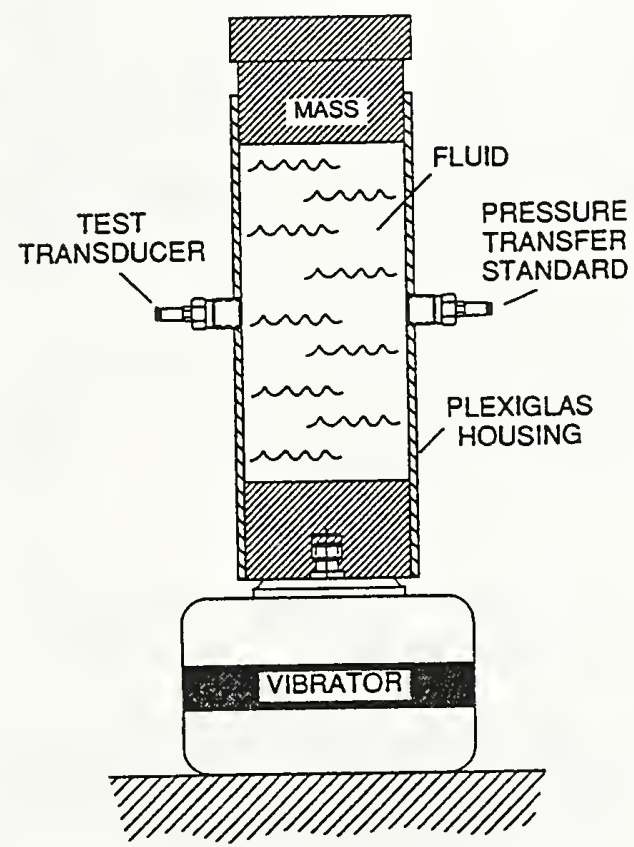

NIST TRACEABILITY - Secondary Pressure Transfer Standard

\section{PNEUMATIC PULSE CALIBRATOR USING}

\section{FAST-ACTING VALVES}

A number of hydraulic and pneumatic pulse calibration devices have been built using manual or solenoid valves. The device at left uses a digital pressure gage to set an accurate static reference pressure. A fast-acting valve quickly switches the pressure sensor to the reference pressure which produces a positivegoing pressure pulse of known amplitude with $5 \mathrm{~ms}$ rise time. It is usually more difficult to produce a positive-going step pulse than a negative pressure drop. Major concern with pulse calibrators involves reducing rarefaction waves in the pressure volume associated with the valves.

\section{VIBRATING COLUMN OF LIQUID}

This calibration method adapts vibration transfer standard techniques to pressure calibration. An electrodynamic shaker vibrates a column of fluid which generates a sinusoidal pressure wave. Output from sensor being calibrated is compared with transfer standard.

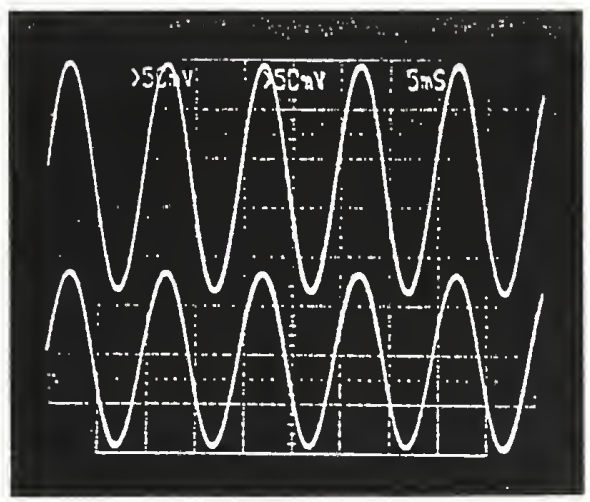



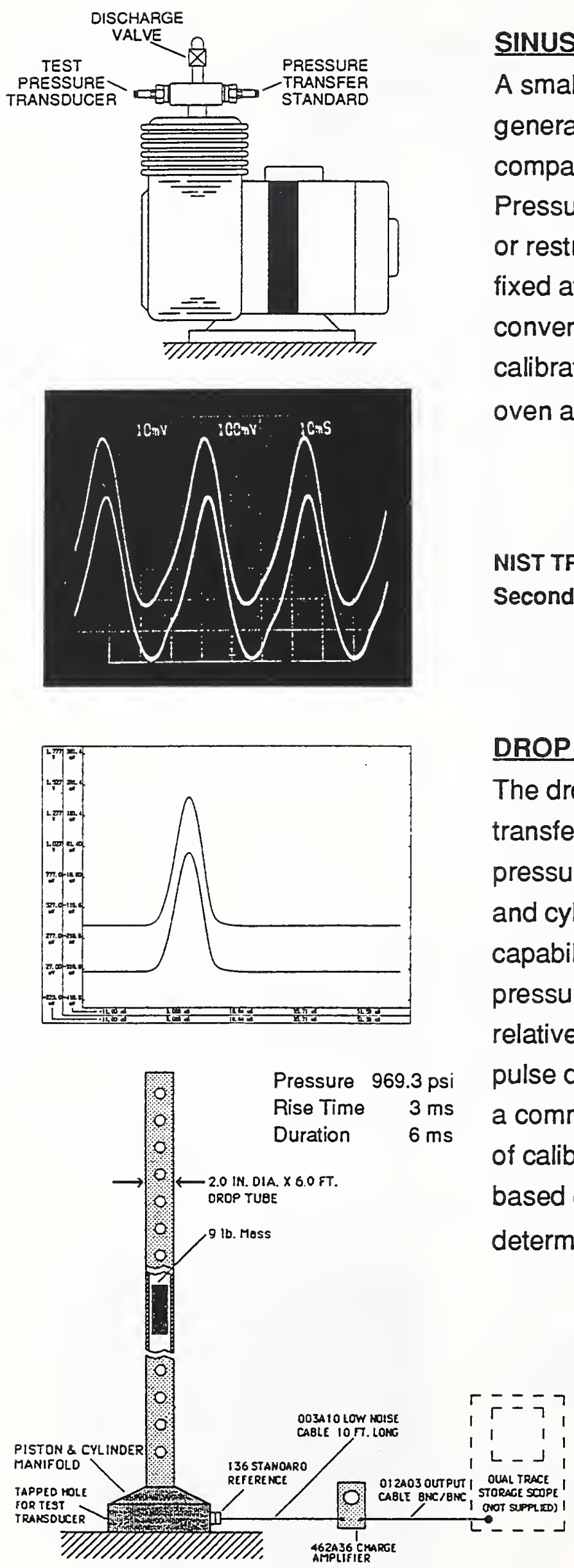

Pressure $969.3 \mathrm{psi}$ Rise Time $3 \mathrm{~ms}$ Duration $6 \mathrm{~ms}$

\section{SINUSOIDAL PRESSURE GENERATORS}

A small air compressor has been modified to generate a repeatable sine wave output for comparison calibration with a transfer standard. Pressure amplitude can be adjusted by opening or restricting the discharge valve. Frequency is fixed at motor speed. This method of calibration conveniently adapts to temperature coefficient calibration by plumbing test transducer inside oven and transfer standard outside.

\section{Pressure Adjustable 3 to $30 \mathrm{psi}$ \\ Frequency Fixed $\quad 29 \mathrm{~Hz}$}

NIST TRACEABILITY - Dynamlcally Calibrated Secondary Transfer Standard.

\section{DROP PULSE CALIBRATOR}

The drop pulse calibrator uses a tourmaline transfer standard to measure the hydraulic pressure pulse from a mass impacting a piston and cylinder manifold. The drop calibrator has capability to generate a wide range of dynamic pressures from about 100 to 20,000 psi with relative ease. Rise time is about $3 \mathrm{~ms}$ and pulse duration $6 \mathrm{~ms}$. Another manufacturer offers a commercial high pressure version of this type of calibrator which uses calculations of mass based on gravity, velocity, and piston area to determine reference pressure.

\section{Impulse Calibrator \\ PCB Model 913A}

NIST TRACEABILITY - Through

Dynamically Calibrated Model 136A

Tourmaline Transfer Standard. 


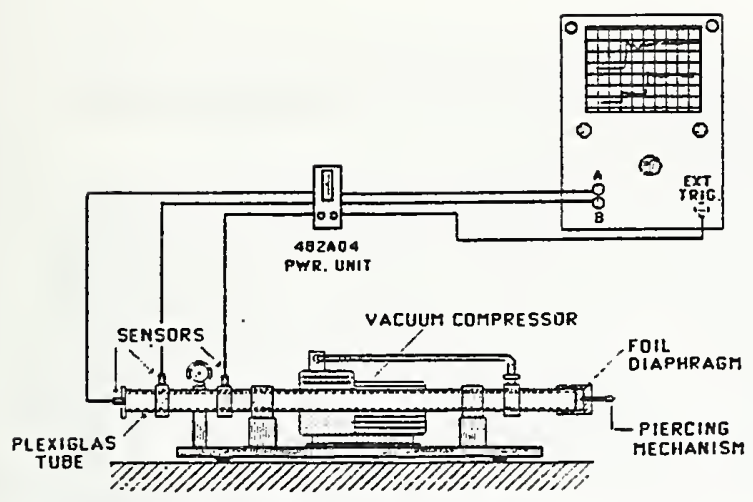

Instrumented Shock Tube, Model 901402

Atmospheric Driven ( $3 \mathrm{ft} . \times 11 / 2$ in. diameter)

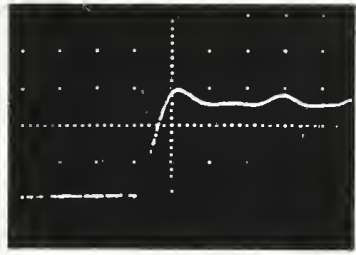

Shock Response from Accel-compensated Transducer

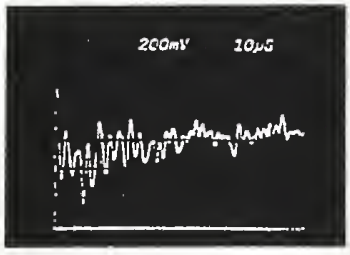

Shock Response from Non-compensated Transducer

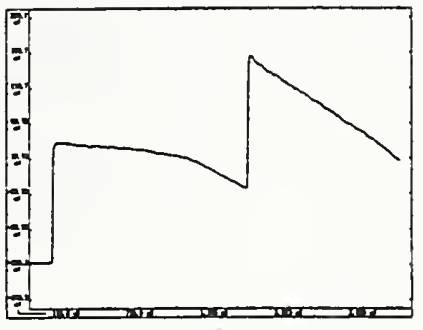

Incident Pressure 113A21 iCP Transducer Pressure 5 psi Rise Time 8

\section{CALIBRATION SHOCK TUBE}

The helium or air-driven shock tube is capable of producing shock waves with nanosecond rise times at pressures from 3 psi using foil diaphragms to $>1,000$ psi with sheet aluminum diaphragms. Shock pressure calibration agrees within $3 \%$ of other calibration methods. A cone shaped adaptor installed at the end of the shock tube test section amplifies reflected pressures to $>10,000 \mathrm{psi}$. The cone adaptor, while not suited for calibration purposes, is useful for developing high pressure, high temperature shock waves for testing thermal and mechanical shock capability of pressure sensors.
134A Pressure Bar

Pressure 123 psi

Rise Time $3 \mu \mathrm{s}$

\section{SHOCK TUBES}

Simple atmospheric-driven plexiglas shock tube generates low pressure shock waves for resonant frequency determination of pressure transducers and gas passages associated wilth recessed diaphragms or recessed mounting. NIST traceability for determination of transducer or passage resonance through electrically calibrated measurement instruments.

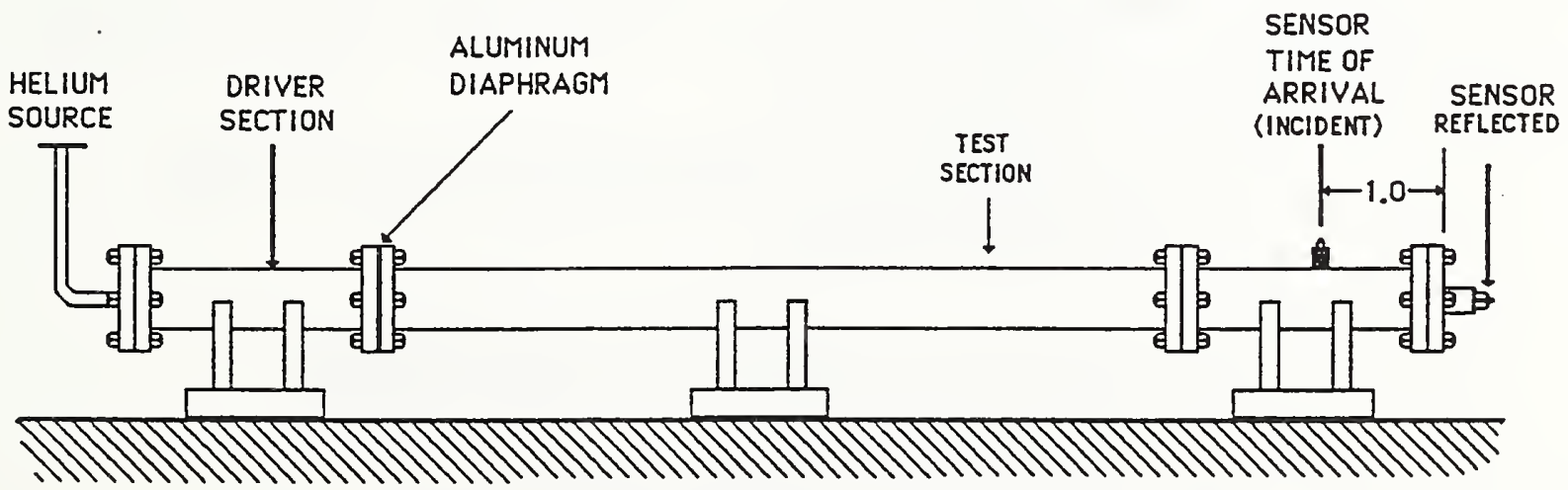

Calibration Shock Tube, Model 901A10

(6 ft. $\times 3$ in. diameter)
Amplitude of shock pressure step is calculated from: Veiocity initial absolute pressure Temperature 


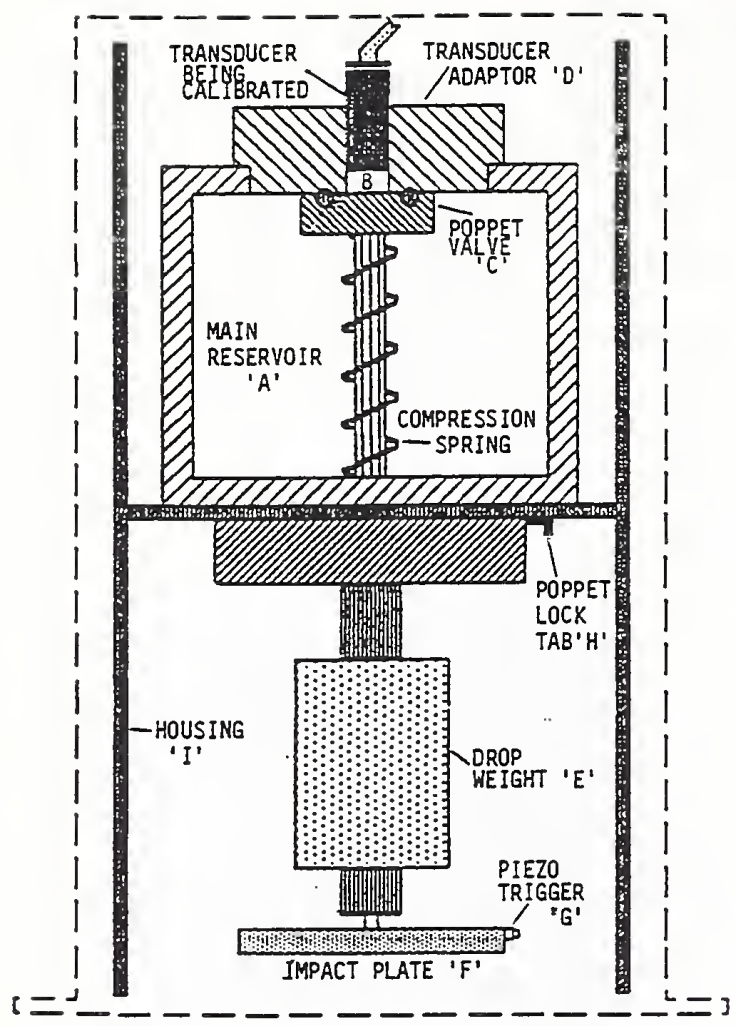

\section{ARONSON SHOCKLESS PRESSURE STEP GENERATOR}

The "Aronson" Shockless Pressure Step Generator is a step toward combining capabilities of several of the special purpose calibrators into one device. Capabilities include the use of high accuracy digital reference pressure standards and fast-acting poppet type valves to produce positive or negative step pressures with rise times approaching those of shock tubes.

The Aronson Step Pressure Generator was developed by Phil Aronson and Robert Waser at the U.S. Naval Ordinance Laboratory. Their primary objective was to develop a device capable of performing dynamic step pressure calibration with greater accuracy, speed and ease than was possible with the shock tube. Phil Aronson dedicated much of his professional career to transient pressure measurements and dynamic calibration. After acquiring a license agreement with the Navy, PCB named the calibration device after Phil who passed away in the early 1980's.

The Aronson Step Pressure Generator consists of:

Main Pressure Reservoir " $A$ "

Transducer Adaptor Plug (three sizes)

Spring-loaded poppet valve (three sizes)

A secondary pressure reservoir " $B$ " at poppet valve/diaphragm interface Impact weight for poppet valve release

Trigger output

Precision Valves for adjusting the gas pressure in both reservoirs

A durable housing and support system

Two each: digital reference pressure gages (optional)

Helium gas pressure source is provided by the customer 
The concept and operation of the Aronson Step Pressure Generator is quite fundamental. It simply involves applying to the transducer an accurately known static pressure very quickly. This is accomplished by pressurizing the main reservoir " $A$ " with an accurately measured static pressure and then exposing the transducer to the reference pressure by releasing the quick-opening poppet valve. The pressure drop in the main reservoir due to the added volume between the transducer diaphragm and poppet valve is negligible with flush diaphragm pressure sensors. Any pressure drop would be indicated by the digital pressure gage which monitors pressure in the reservoir.

The "Aronson" Step Pressure Generator has two separate pressure reservoirs $(A \& B)$ in which the pressure is controlled by accurate static digital pressure gages. In addition to the main pressure reservoir " $A$ ", pressure between the poppet valve and the diaphragm of the transducer (reservoir "B") can be pressurized with a known static pressure. Having control of a known pressure in each reservoir offers unique capability to compare static vs. dynamic response, and provide small incremental pressure steps at higher static levels.

Rise time of a step pressure depends on:

1. type of gas used (helium recommended for fastest rise time)

2. diameter of poppet valve

3. initial pressure difference across the poppet valve

4. design of transducer diaphragm (flush or recess)

Traceability to NIST is through calibration of accurate DC reference gages, used to set the known static pressure level which the transducer under calibration is being rapidly switched to. 


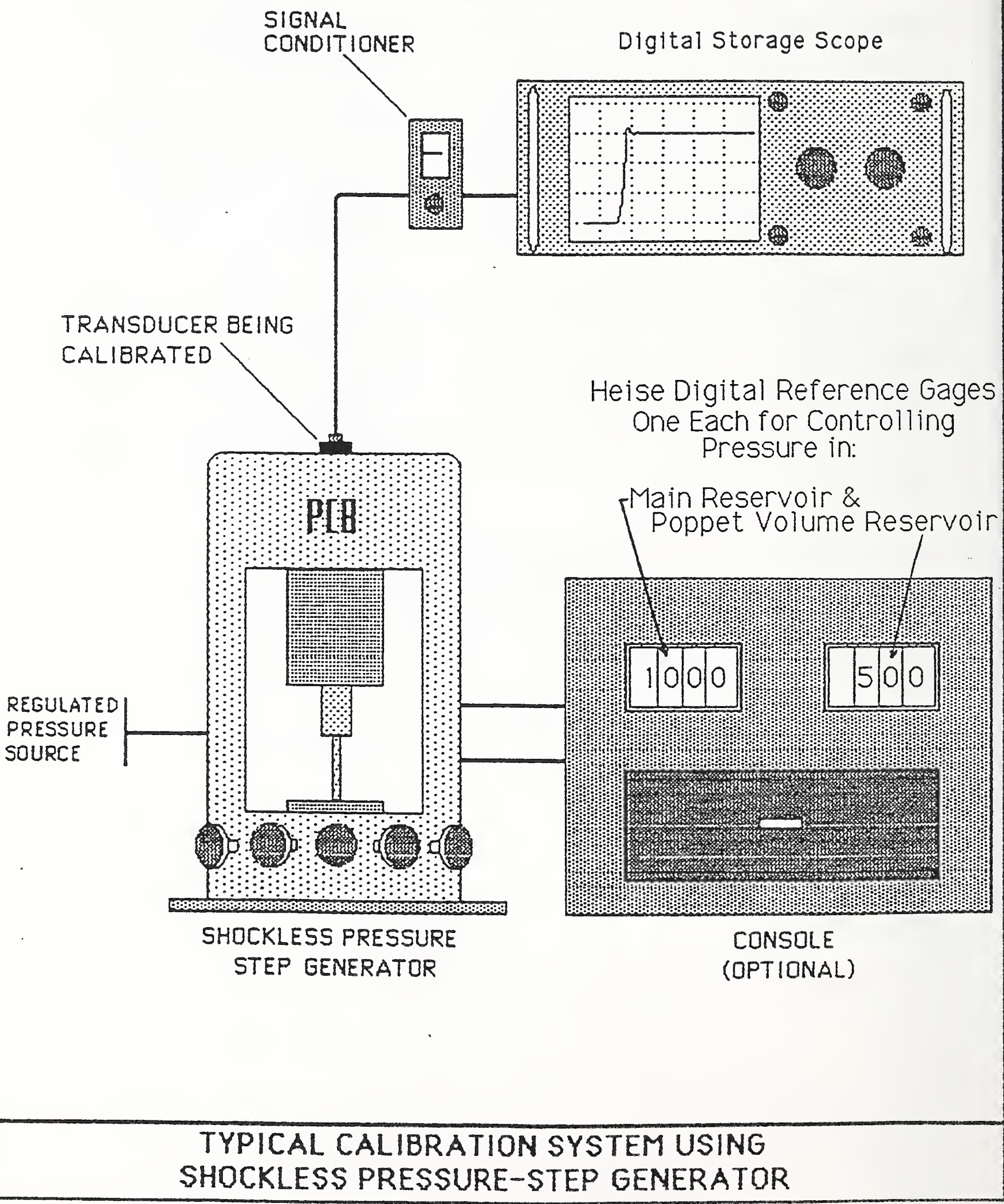




\section{ARONSON SHOCKLESS PRESSURE-STEP GENERATOR Model 907A}

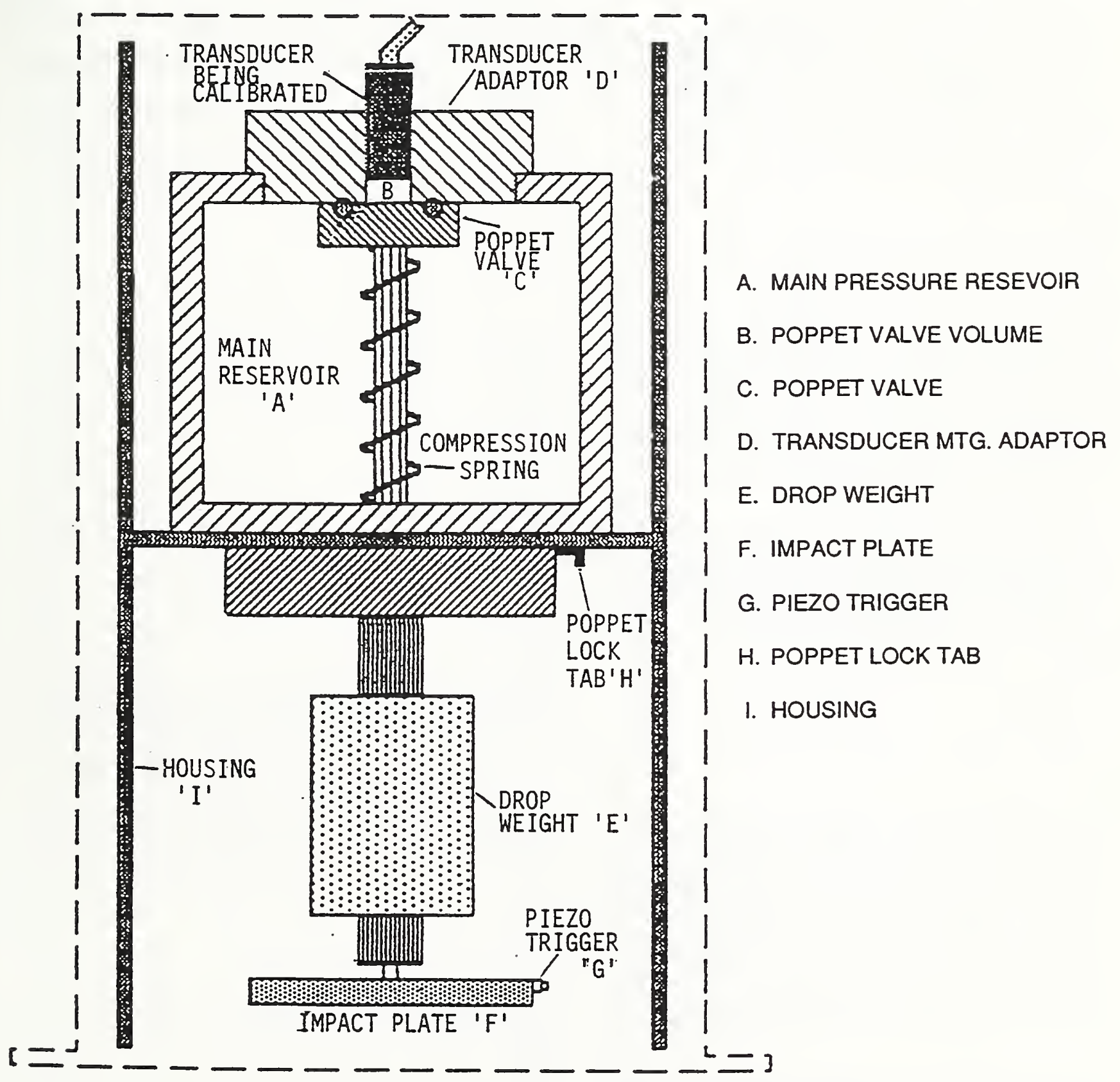


1. Dynamic calibration of pressure transducers. Oscillations of $71 \mathrm{k} \mathrm{Hz}$ at peak of the pressure is due to the rarefaction waves at the interface of the poppet valve and transducer diaphragm.

Dynamic calibration of 113A24 ICP Pressure Transducer using Aronson Step Pressure Generator:

$\begin{array}{ll}\text { Pressure } & 1000 \mathrm{psi} \\ \text { Transducer Sensitivity } & 5.3 \mathrm{mV} / \mathrm{psi}\end{array}$

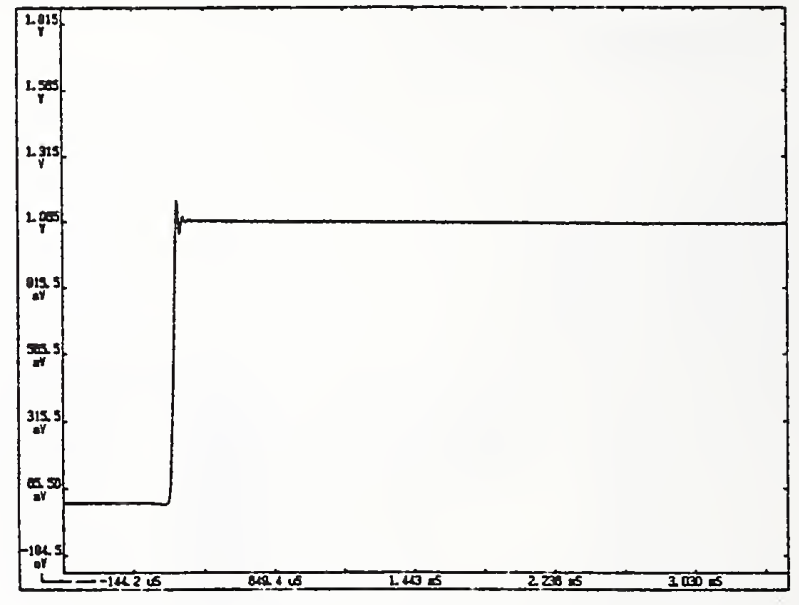

2. Determination of the RC time constant of an entire pressure measurement system including the transducer, signal conditioner and readout.

Discharge Time Constant (DTC)

Time required for a transducer or measurement system to discharge its signal to $37 \%$ of the original value from a step change of pressure.

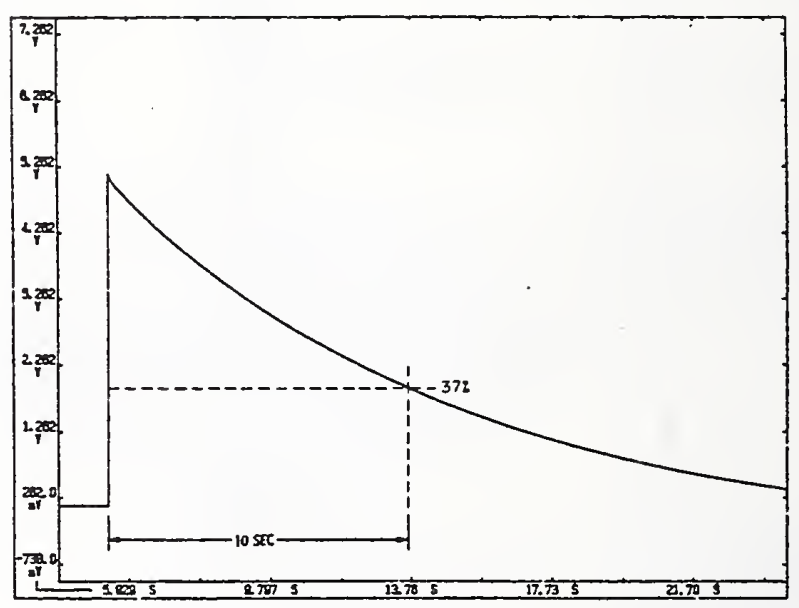

3. Determination of response time and resonance of recessed cavities and passages. Model 113A24, ICP®Pressure Transducer recess mounted 0.050 inch with 150 psi step change in pressure.

Resonant frequency of 0.050 recessed passage Frequency $24.8 \mathrm{k} \mathrm{Hz}$ Rise Tiem $\quad 71 \mu \mathrm{s}$ Pressure Step 150 psi

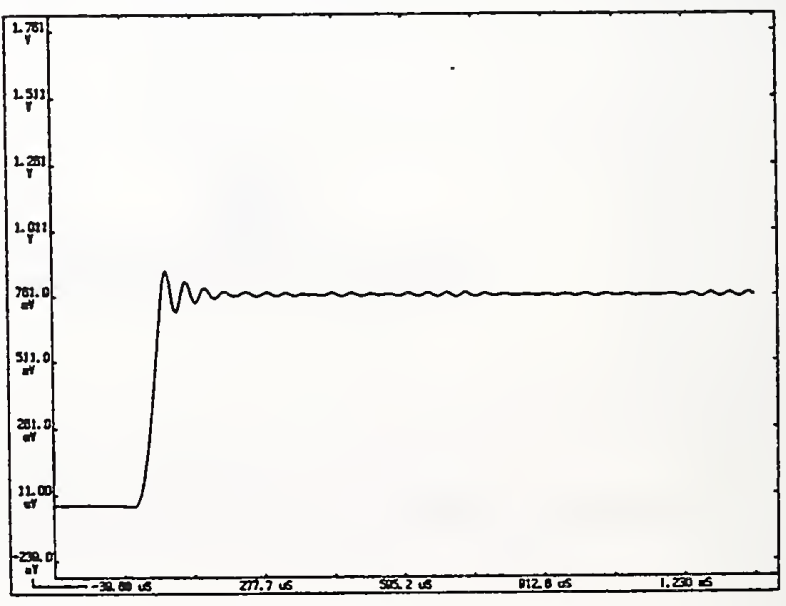


4. Calibration of incremental pressure steps above or below preset static levels. It is possible to simulate the static pressure environment on the sensor diaphragm that might be encountered in an underwater environment while applying a step pressure of known amplitude.

5. Comparison of static and dynamic calibration of a pressure transducer using the same pressure amplitude in the same device without moving the transducer.

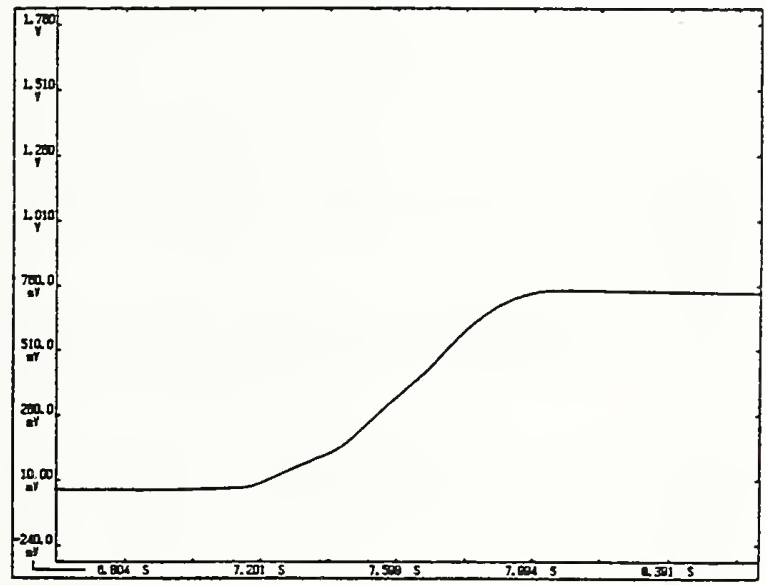

Slowly applied pressure, 150 psi

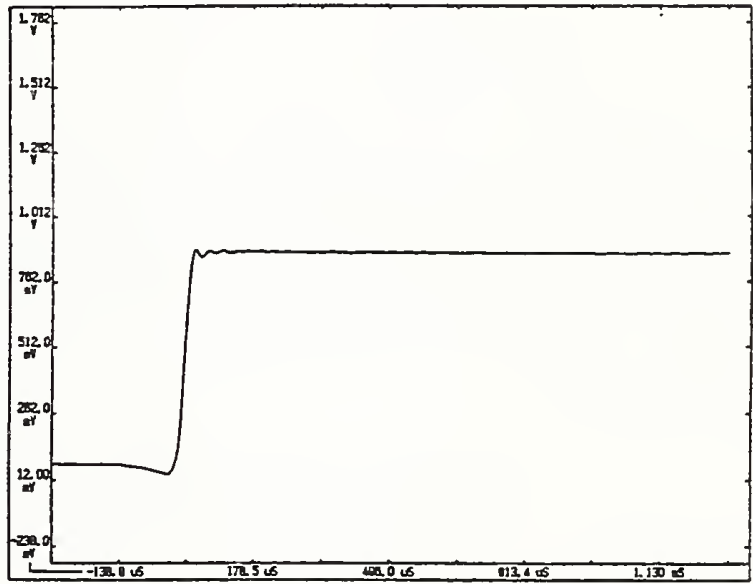

Step pressure of same 150 psi

Rise time $<50 \mu \mathrm{s}$

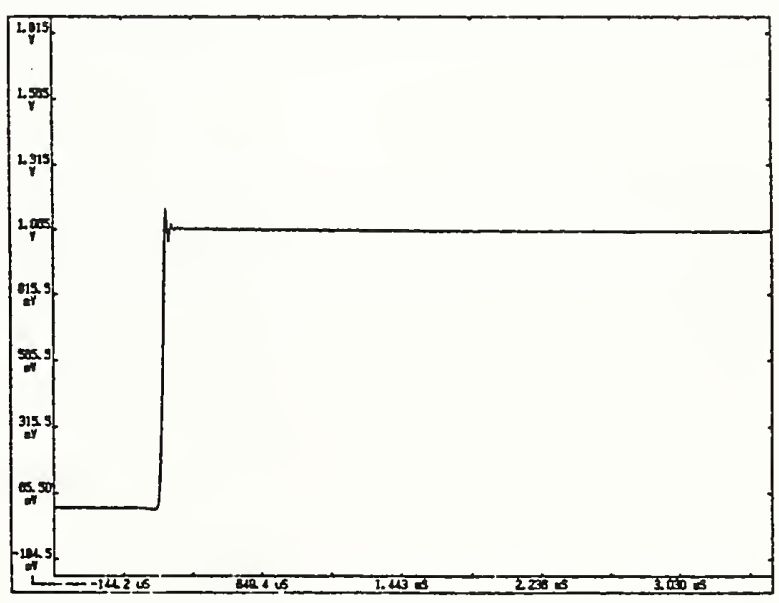

Dynamic calibration of 113A24 ICP Pressure Transducer using Aronson Step Pressure Generator $1000 \mathrm{psi}, 5.3 \mathrm{mV} / \mathrm{psi}$ 
7. Vacuum step pressure calibration.

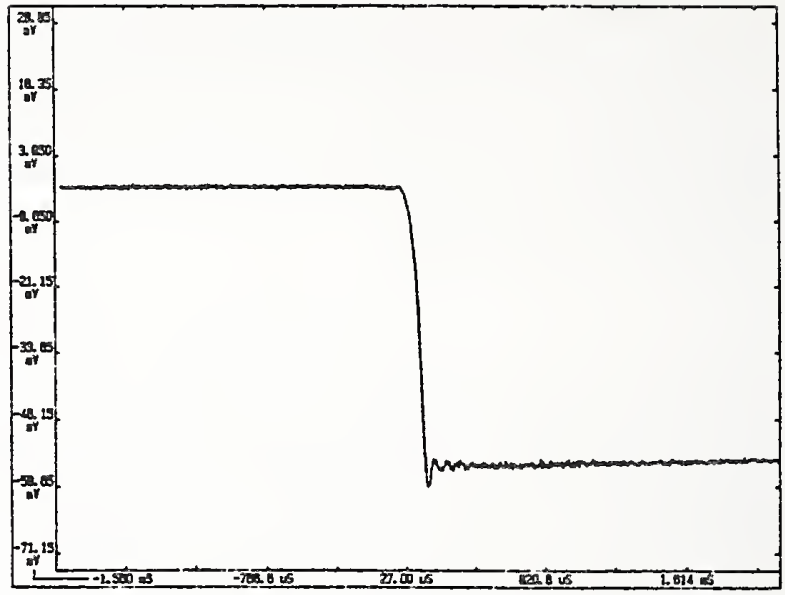

Vacuum step pressure calibration, Model 113A24 ICP Pressure Transducer, -10 psi, $5.34 \mathrm{mV} / \mathrm{psi}$

8. Checkout and troubleshooting of $d y-$ namic pressure transducers, expecially those used for shock and blast wave measurements.

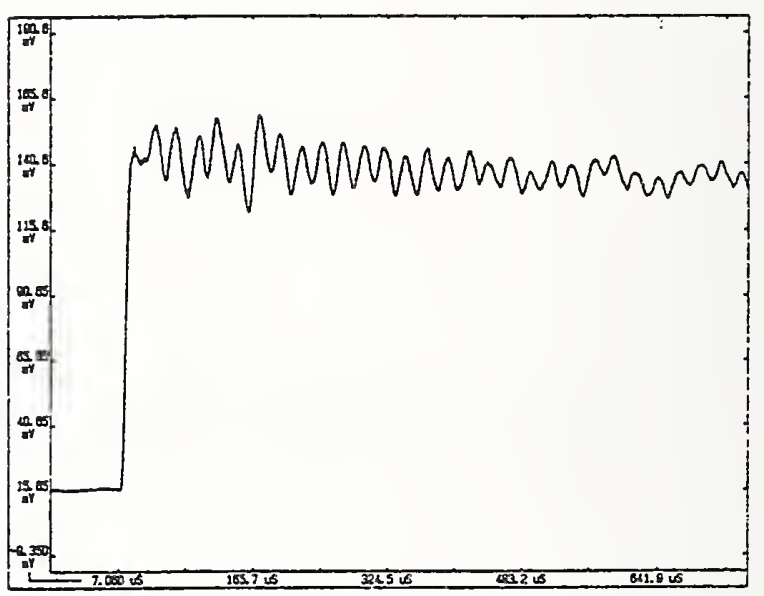

Step pressure calibration detects noise created by defective transducer diaphragm.

9. Pulse calibration detects signal breakup due to erratic connection and resultant zero shift.

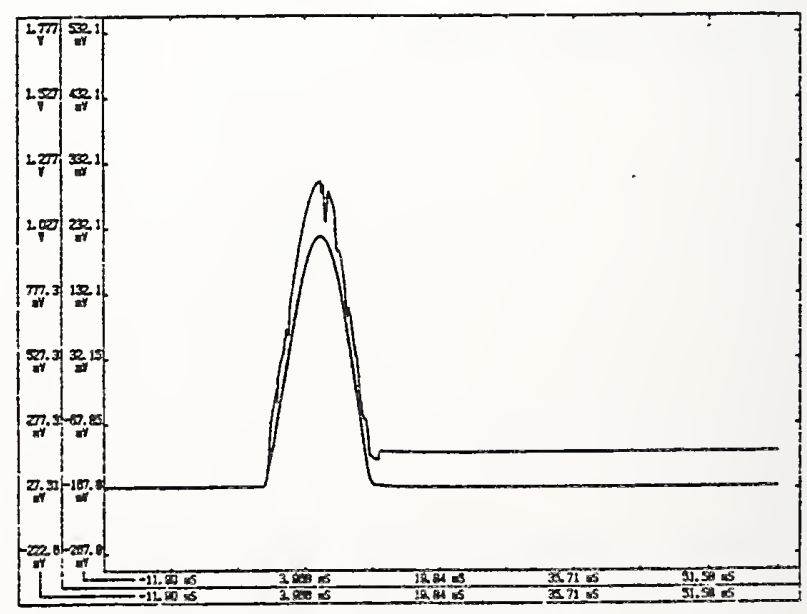


During the process of accumulating calibration data for this report, sensitivity of Model 113A24 ICP $\$$ Pressure Transducer was recorded and plotted using five different methods of calibration. Sensitivity deviations for the methods was less than $\pm 11 / 2 \%$.

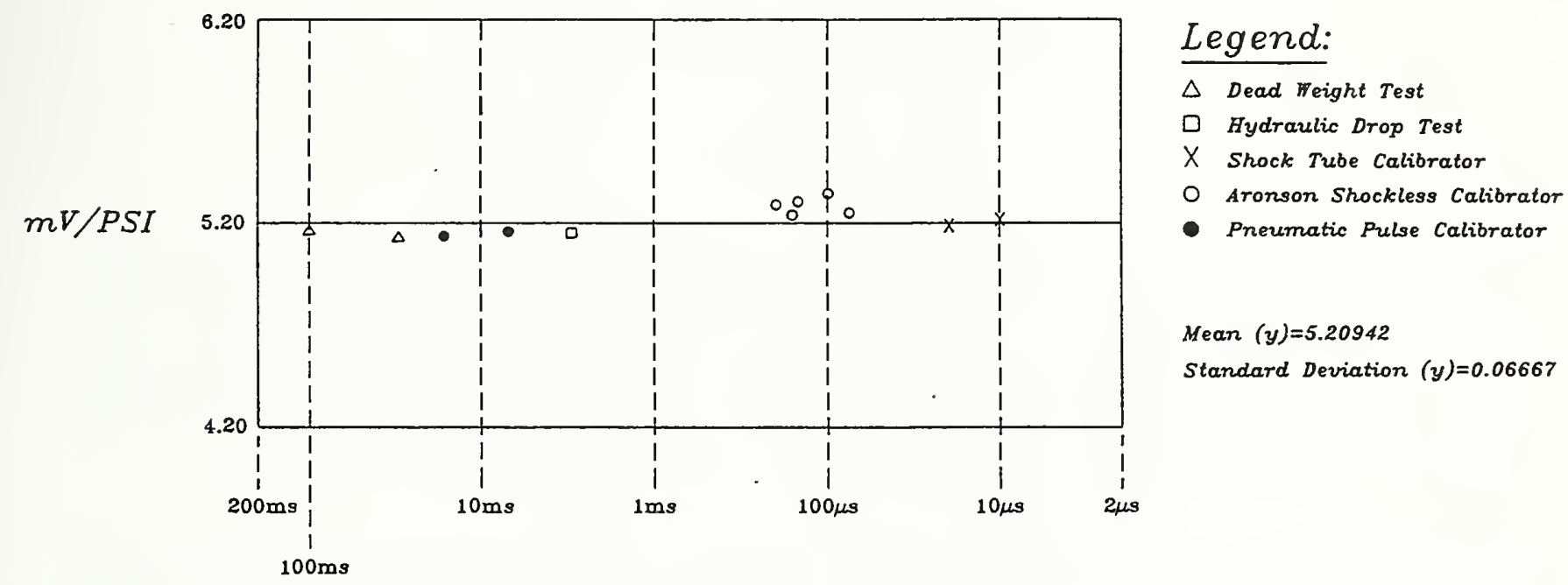

Calibrations of a 113A24 Quartz Transducer using five different calibrators.

While the Aronson Calibrator has not equaled the performance capability of the "Ultima" calibrator, it is a step in that direction. The digital storage oscilloscope has significantly improved the accuracy of dynamic calibration. Dynamically calibrated pressure transfer standards have also contributed to improving the accuracy of sinusoidal and drop-test impulse calibrations.

\section{$\diamond \diamond \diamond \diamond$}

Acknowledgement:

The author would like to thank Ben Granath for providing most of the test results used in this paper.

(8) ICP is a registered trademark of PCB. 


\author{
Improved Accuracy in Piezoelectric Measurements \\ Using a \\ Low Noise, Ultra-High Isolation Signal Conditioner
}

\author{
by \\ Bruce Hartenbaum \\ $\mathrm{H}-\mathrm{Tech}$ Laboratories, Inc. \\ California Institute of Technology \\ James Morrison \\ Lawrence Livermore Laboratory \\ John Gord \\ NavDesign
}

Commercial piezoelectric pressure transducers and accelerometers are used mainly in either the voltage or charge mode. Two shortcomings of existing commercial signal conditioners are: (1) Their single-ended circuitry has zero common mode noise rejection capability, and (2) if the transducer test point is at a different ground potential from data records, several undesirable conditions can occur, including noise feedthrough, voltage offsets in the data waveforms, burnout of the transducer and/or the data records, and shock hazard.

As a result, noise contamination of piezoelectric data often degrades the accuracy of the data. In many cases spectral analysis is incapable of sorting the true signal from the noise. In other cases the data are filtered with attendant loss of signal components. The resultant data are misleading.

We have developed a new piezoelectric transducer signal conditioner that under actual field conditions reduces noise contamination by 20 to $60 \mathrm{db}$ below that of conventional systems. The new signal conditioner employs hybrid opticalelectronic circuitry, while maintaining full compatibility with commercial transducers and most data transmission systems. The new signal conditioning has enabled unusually clean data to be recorded at full bandwidth in noisy environments. Optical decoupling provides $120 \mathrm{db}$ of isolation at $100 \mathrm{Khz}$ and up to 2500 VRMS potential difference between measurement point and data records. Differential conversion provides $60 \mathrm{db}$ of common mode noise rejection and allows conventional, twisted pair, long signal lines to be driven at their bandwidth limit, i.e., $60 \mathrm{kHz}$ for low cost cable and $500 \mathrm{khz}$ for low loss cable. In noisy environments, the signal to noise ratio is typically $63 \mathrm{db}$. An additional improvement in measurement accuracy is provided by an end-to-end precision square wave voltage calibrator, either under manual or computer control.

Used in shock and vibration testing, ordnance testing, shock tube research, and biomedical research, the new signal conditioner has been shown to be quieter than either conventional piezoelectric or piezoresistive systems. An important benefit to NIST is measurement accuracy. An additional benefit to commercial users is decreased set-up time and lower test costs. 


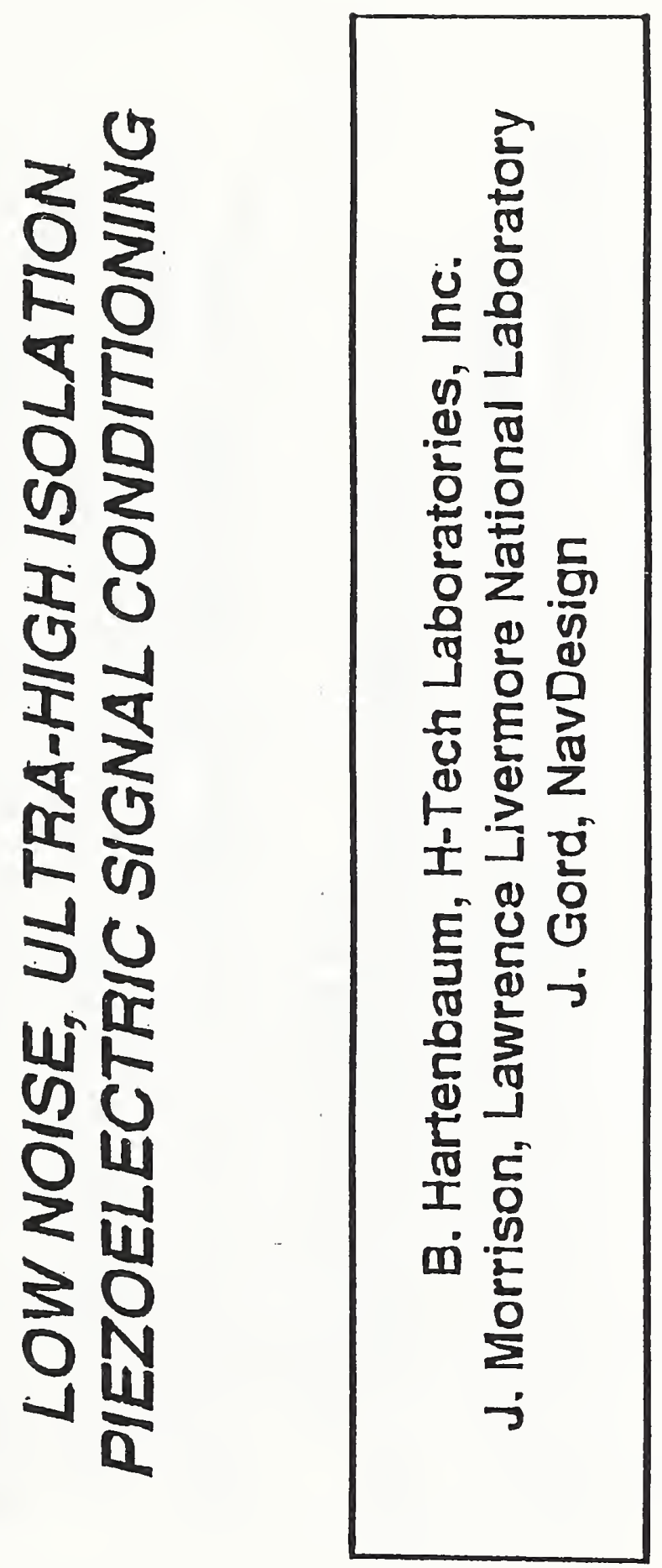




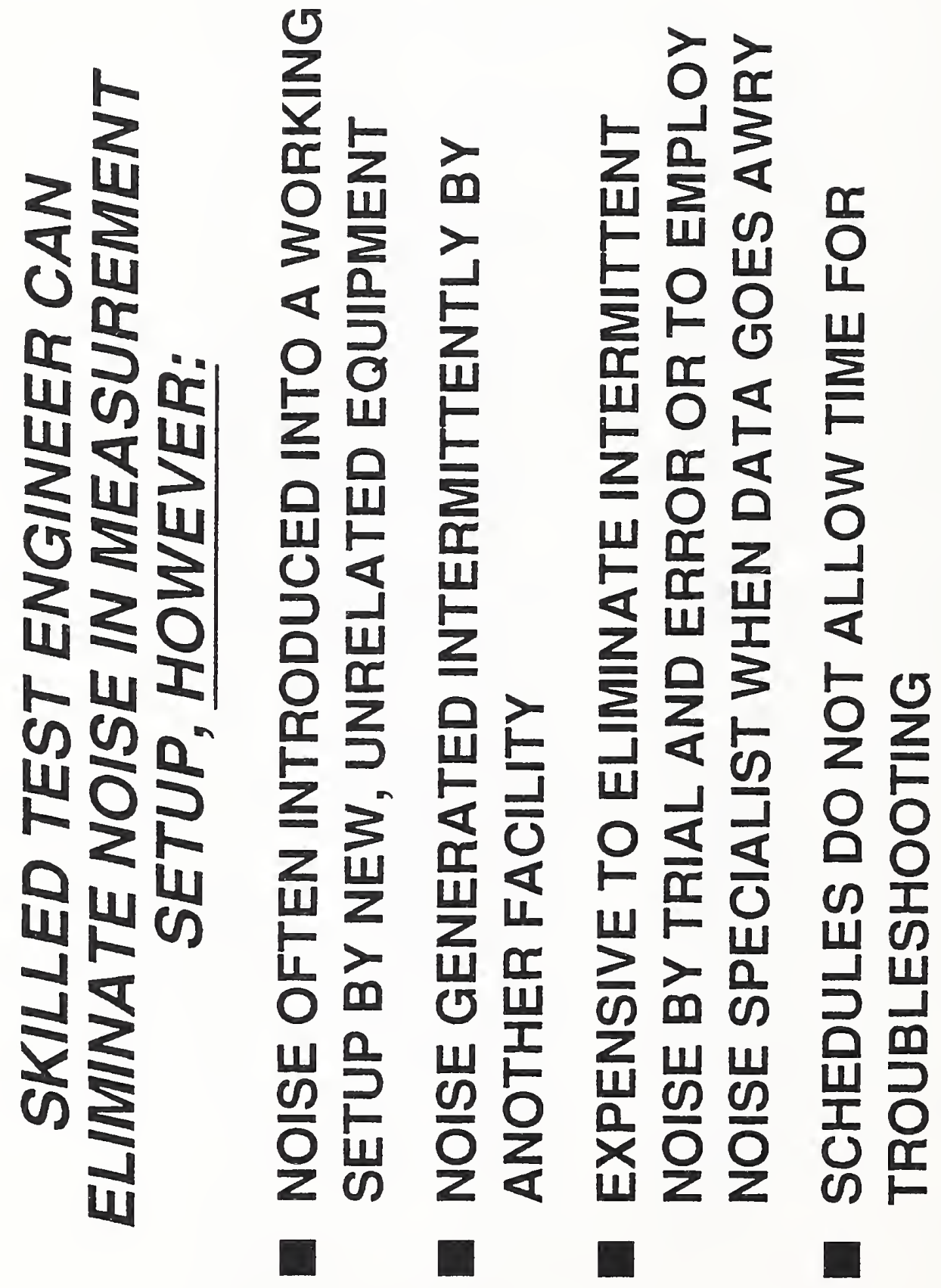




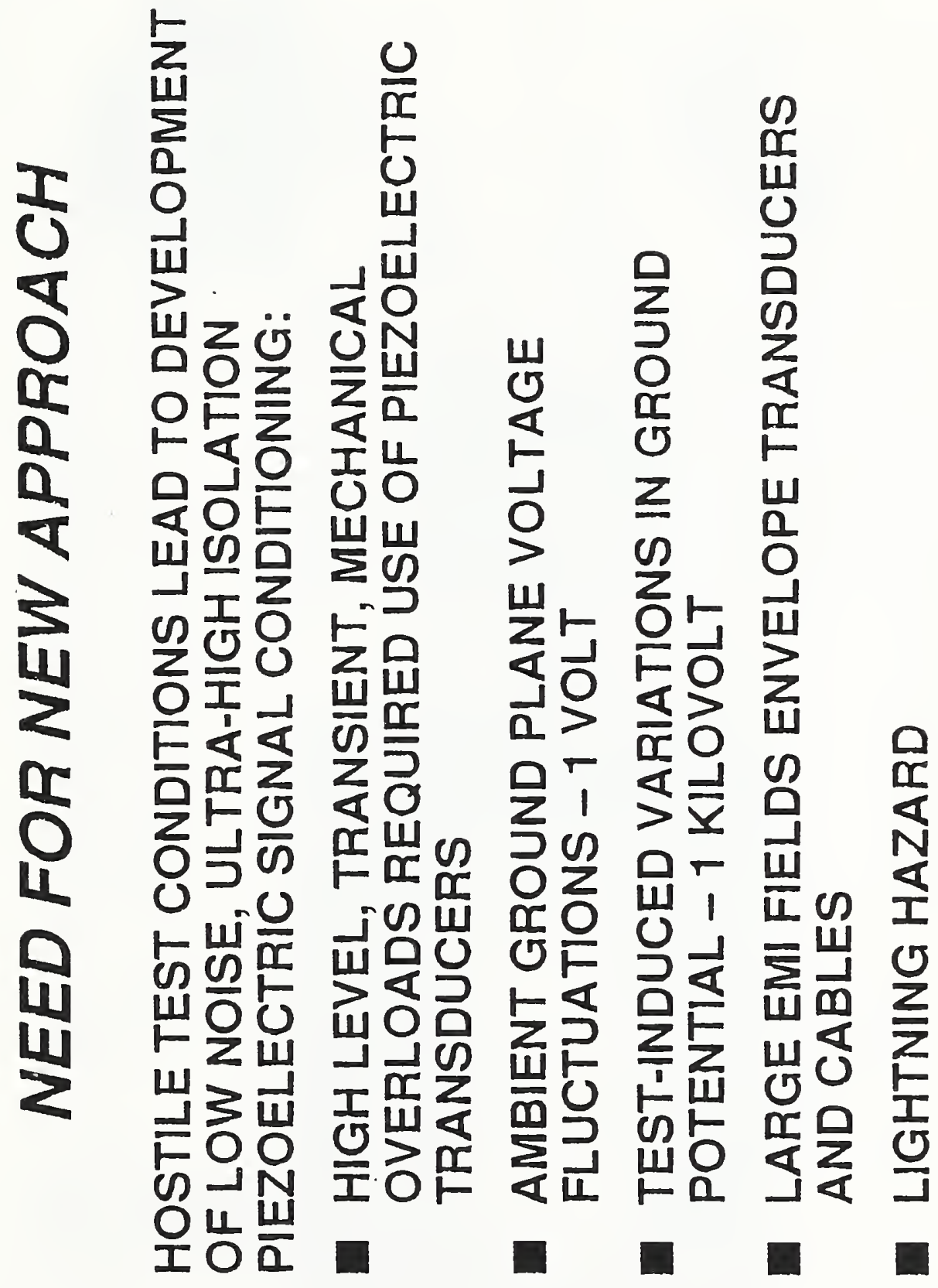



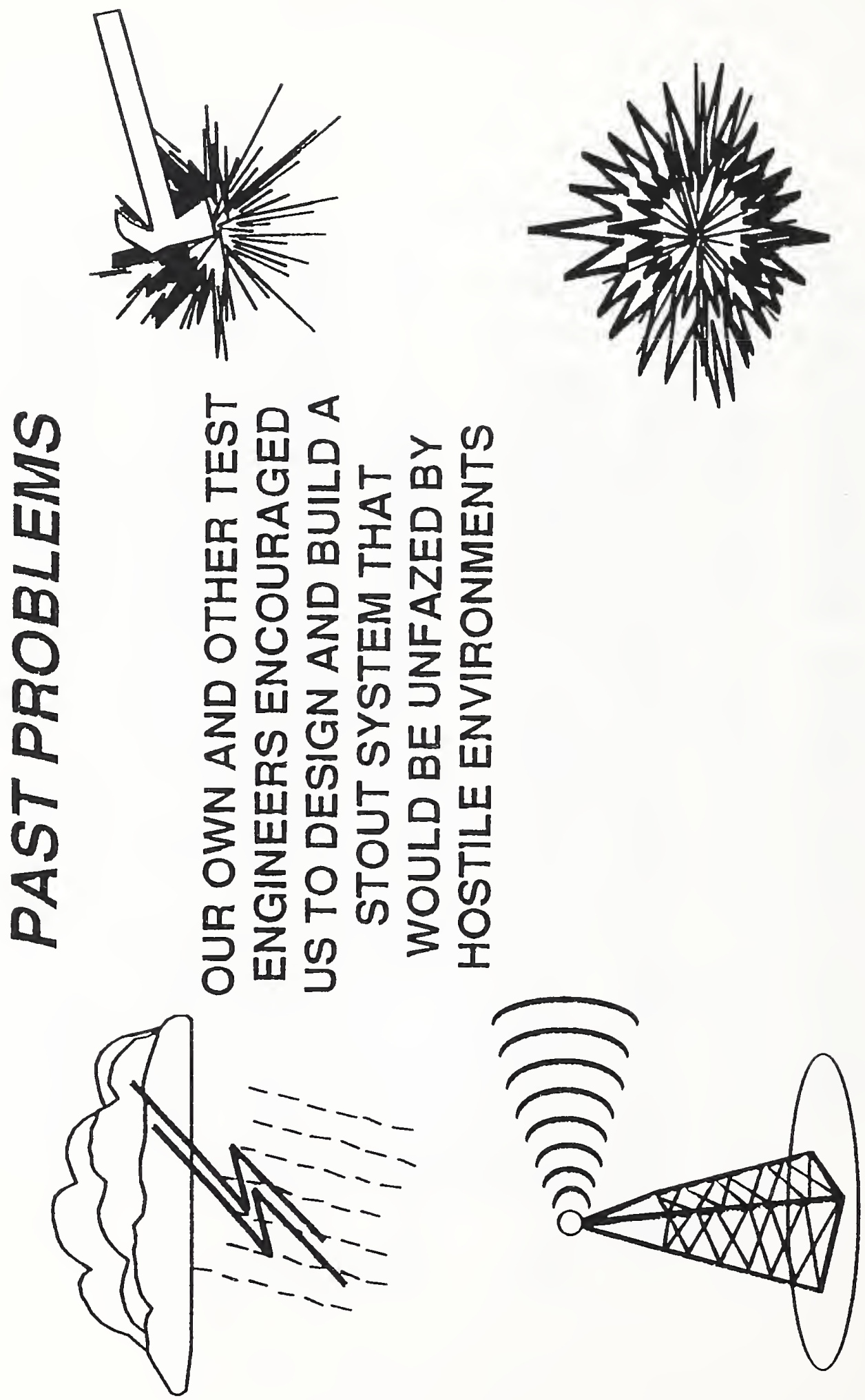


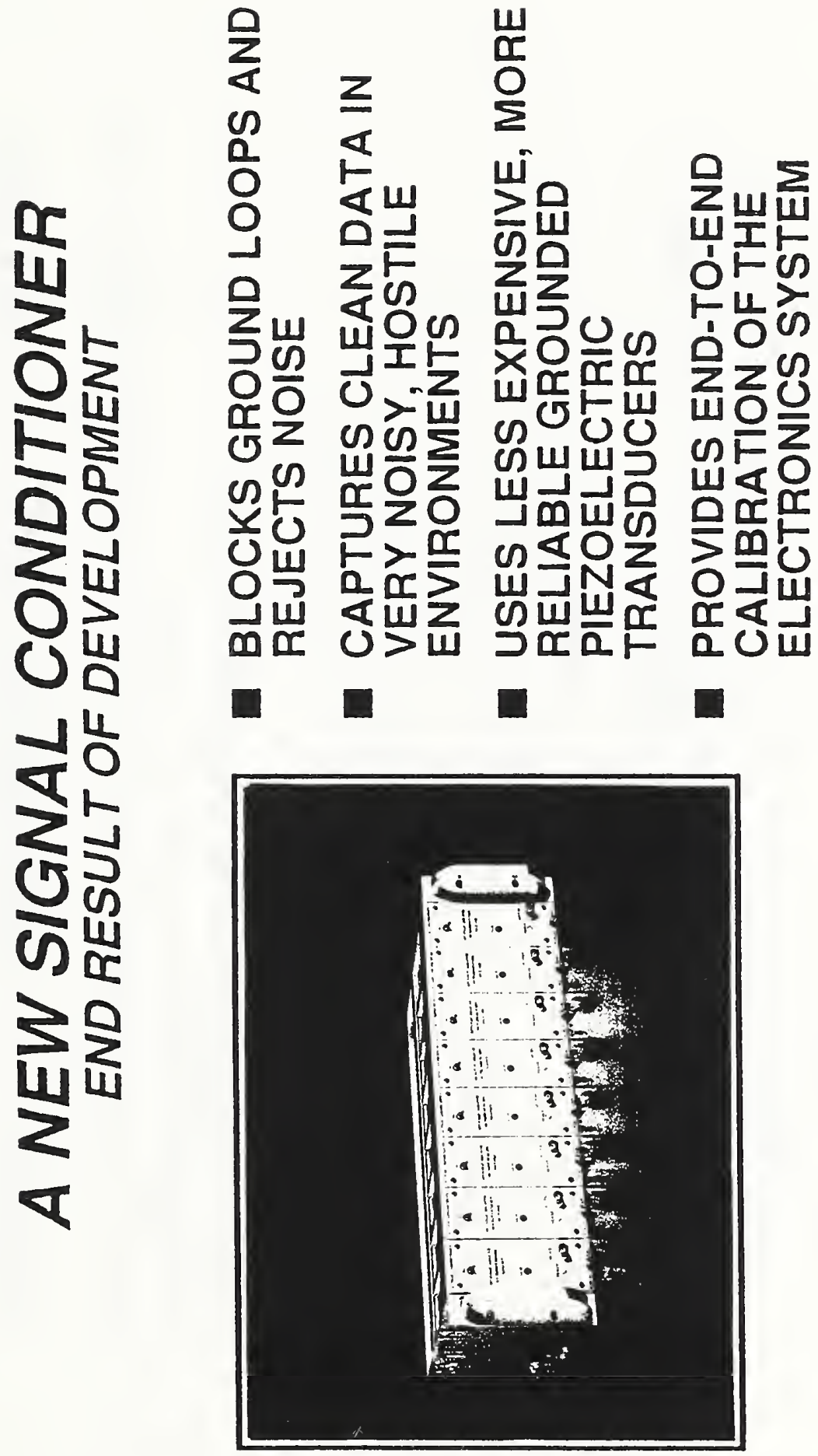




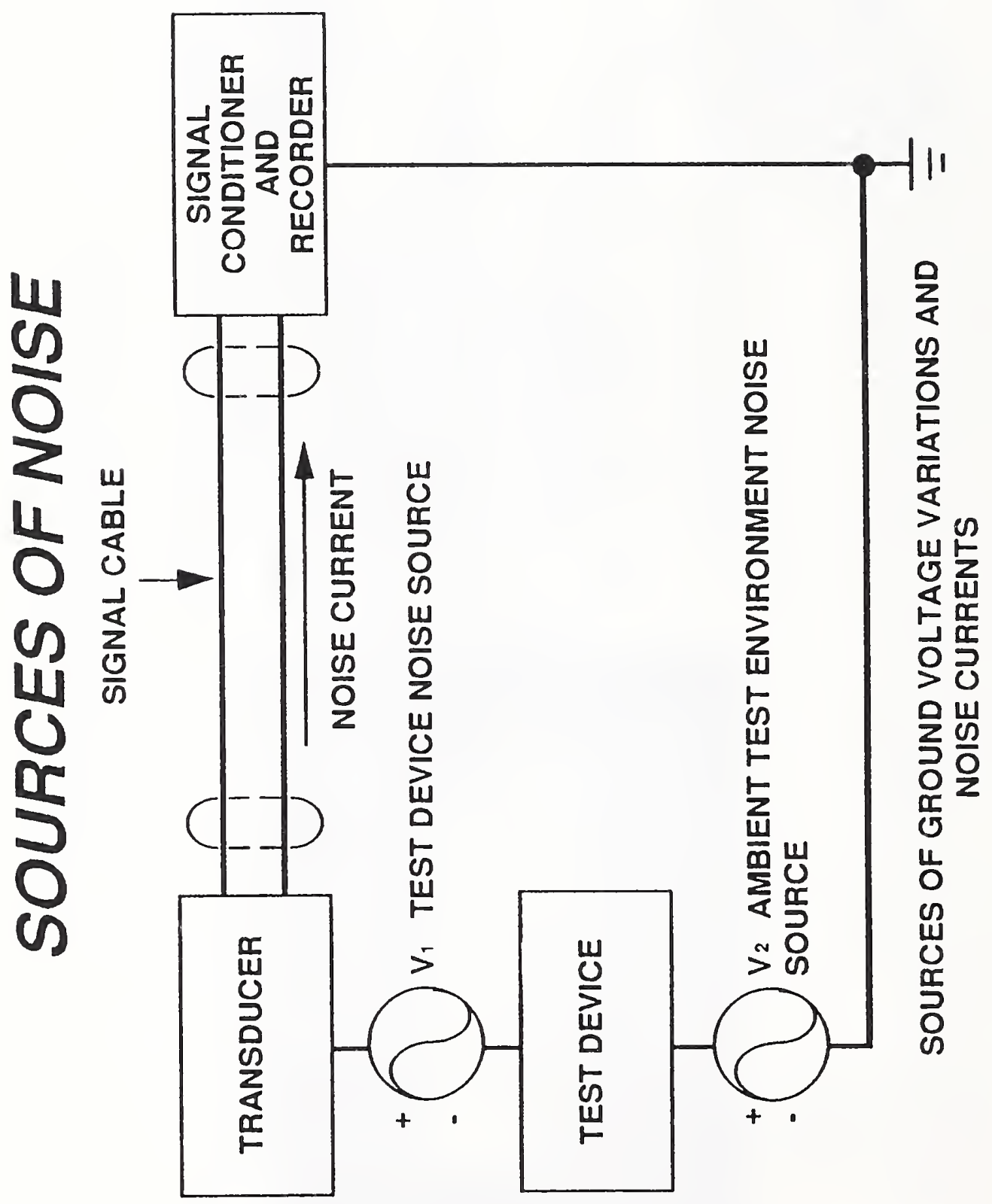




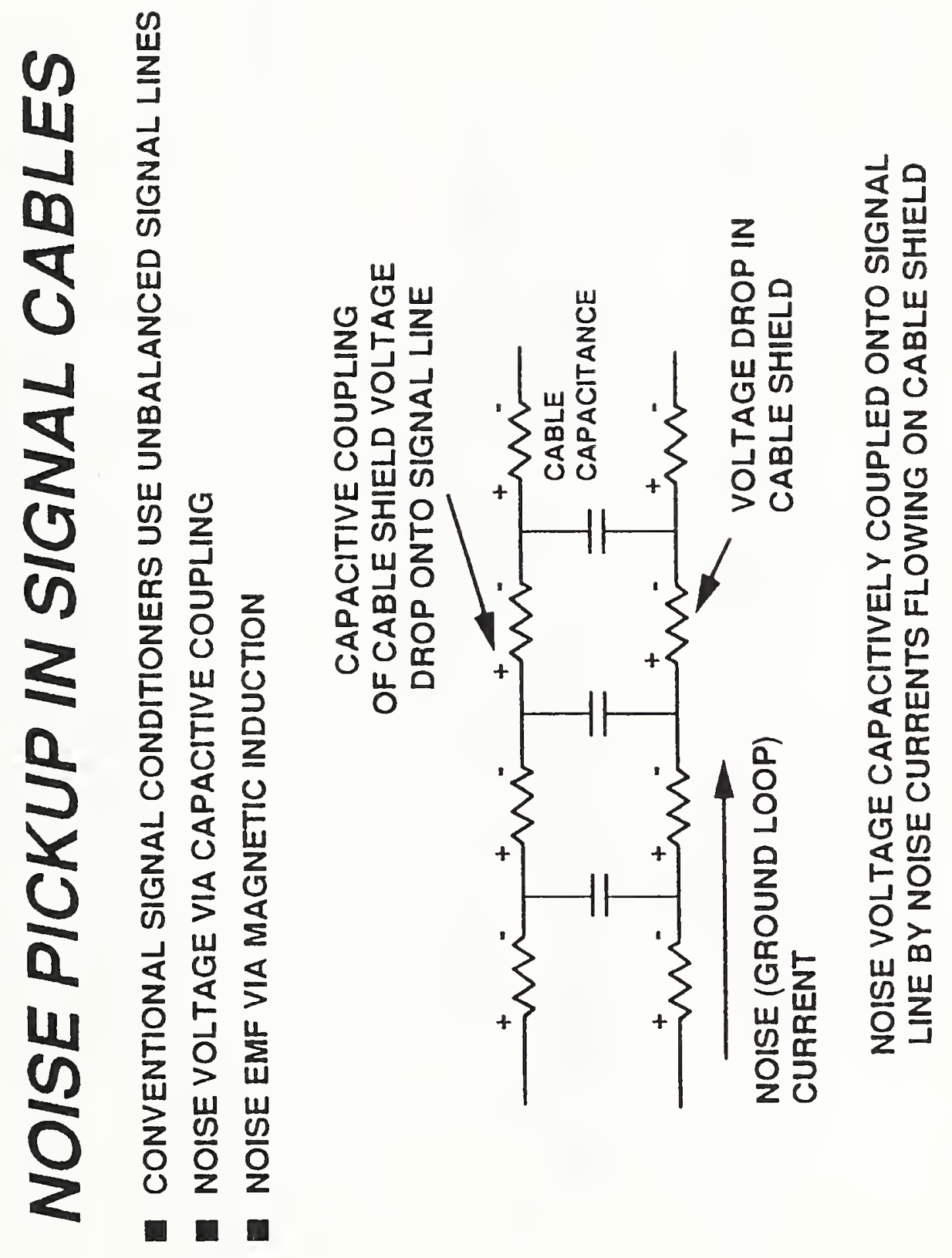




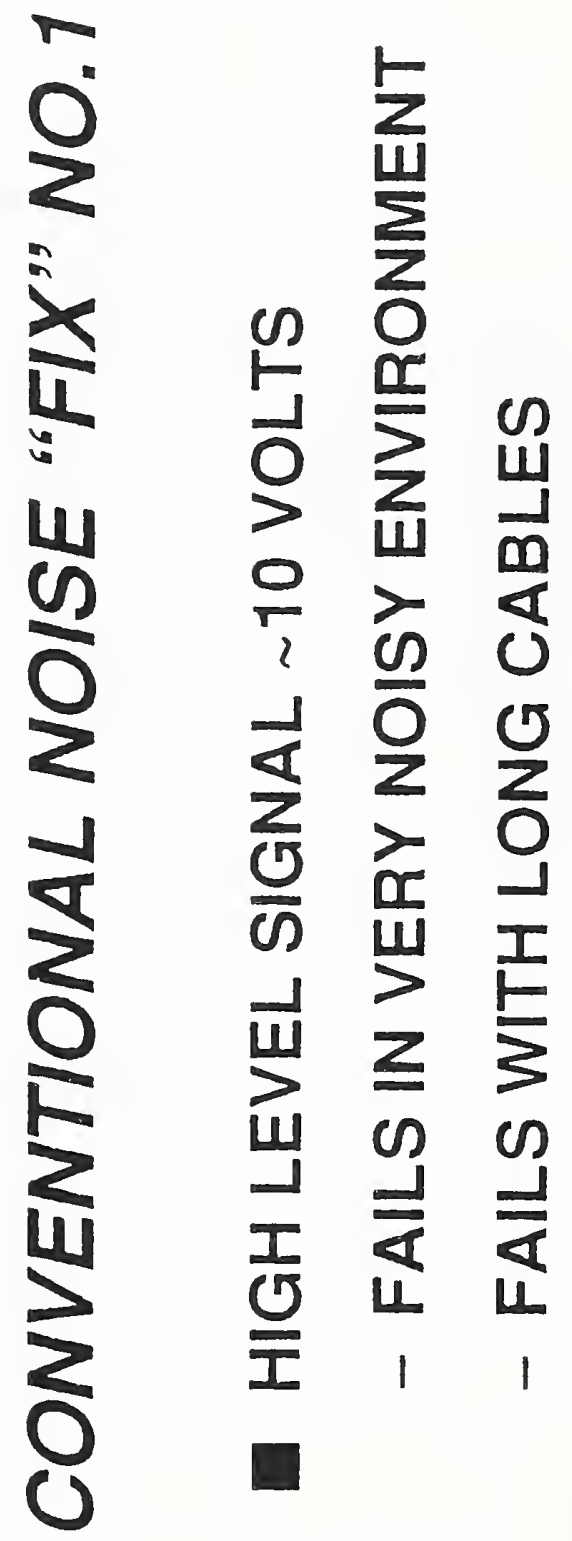




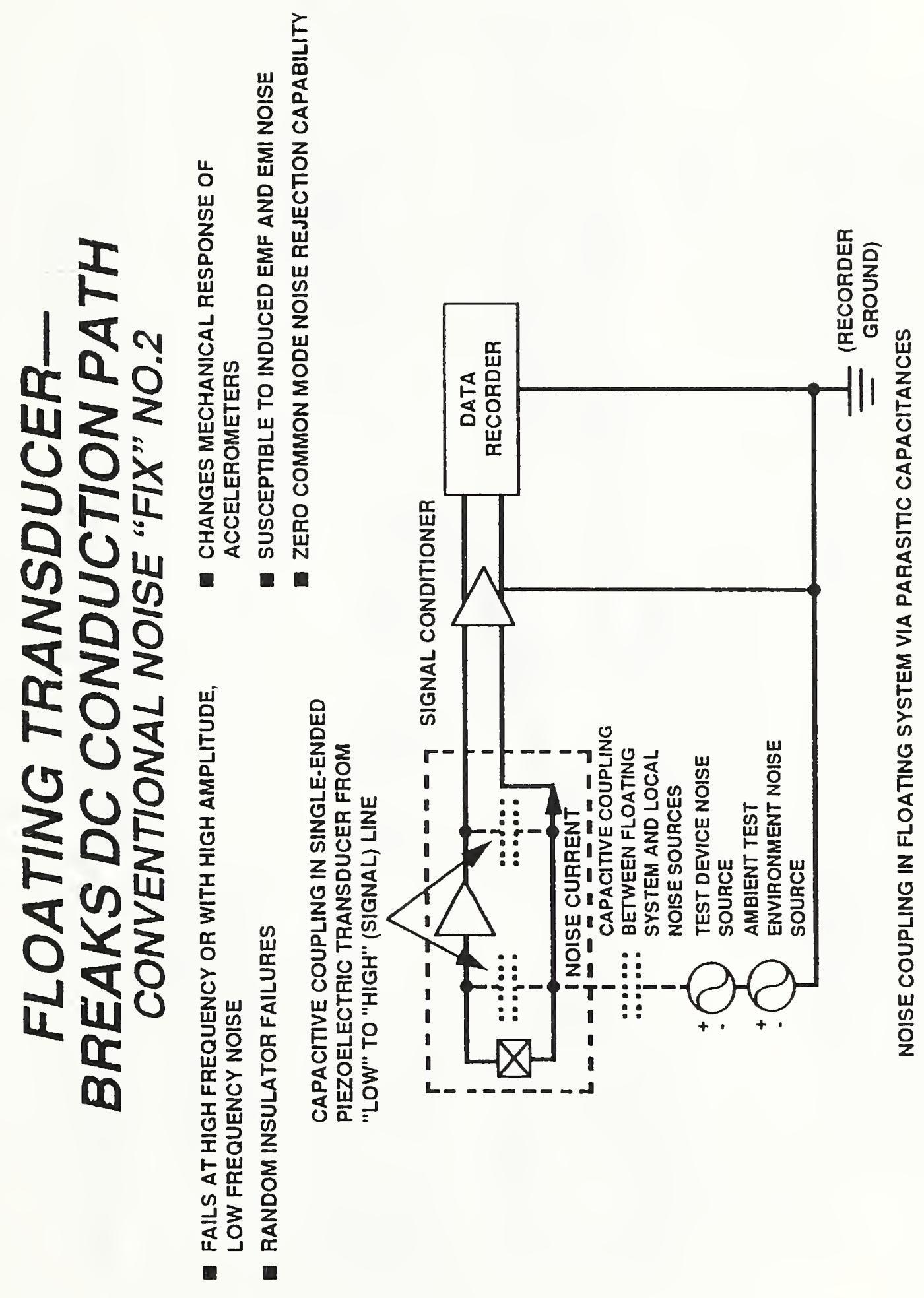




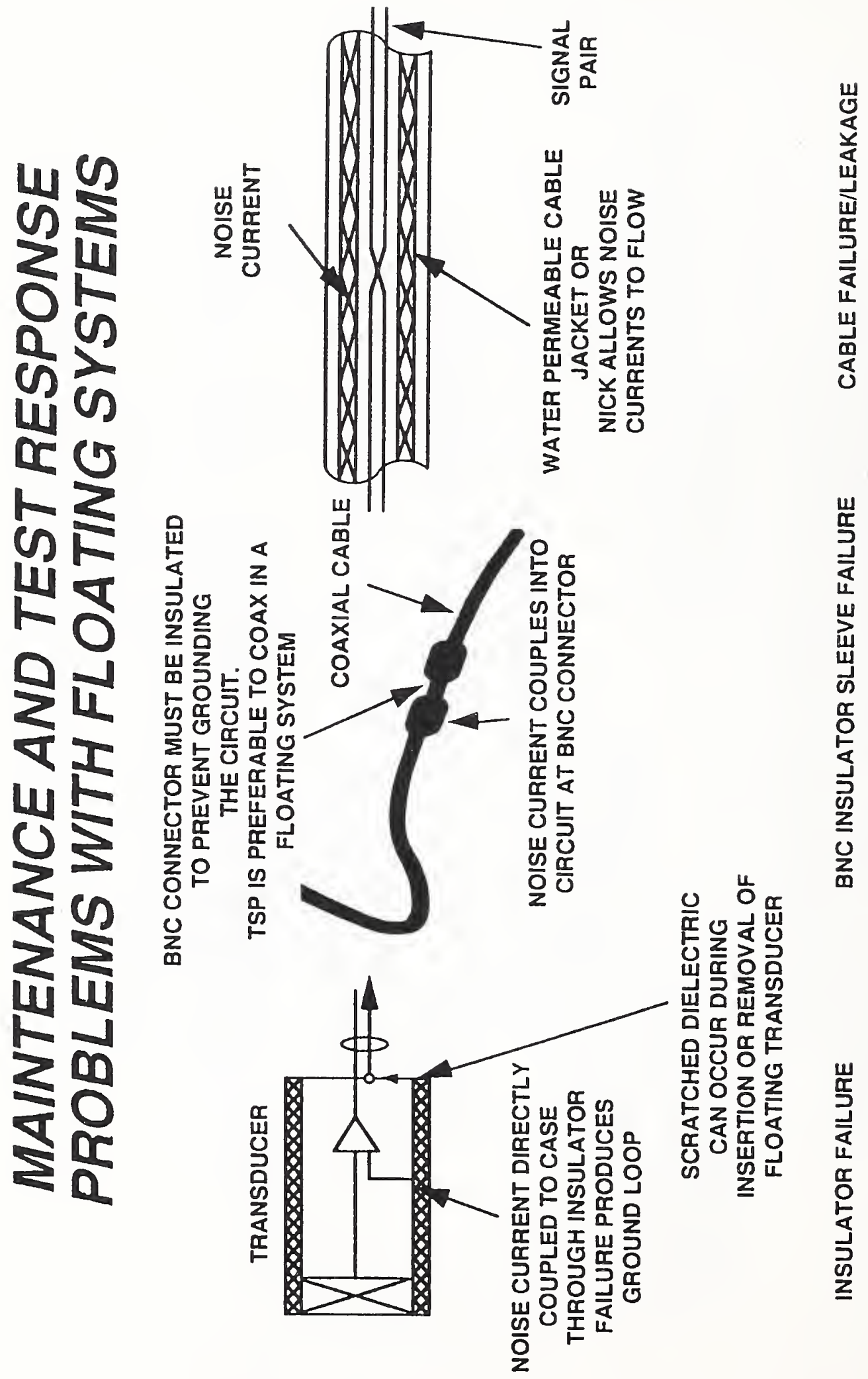




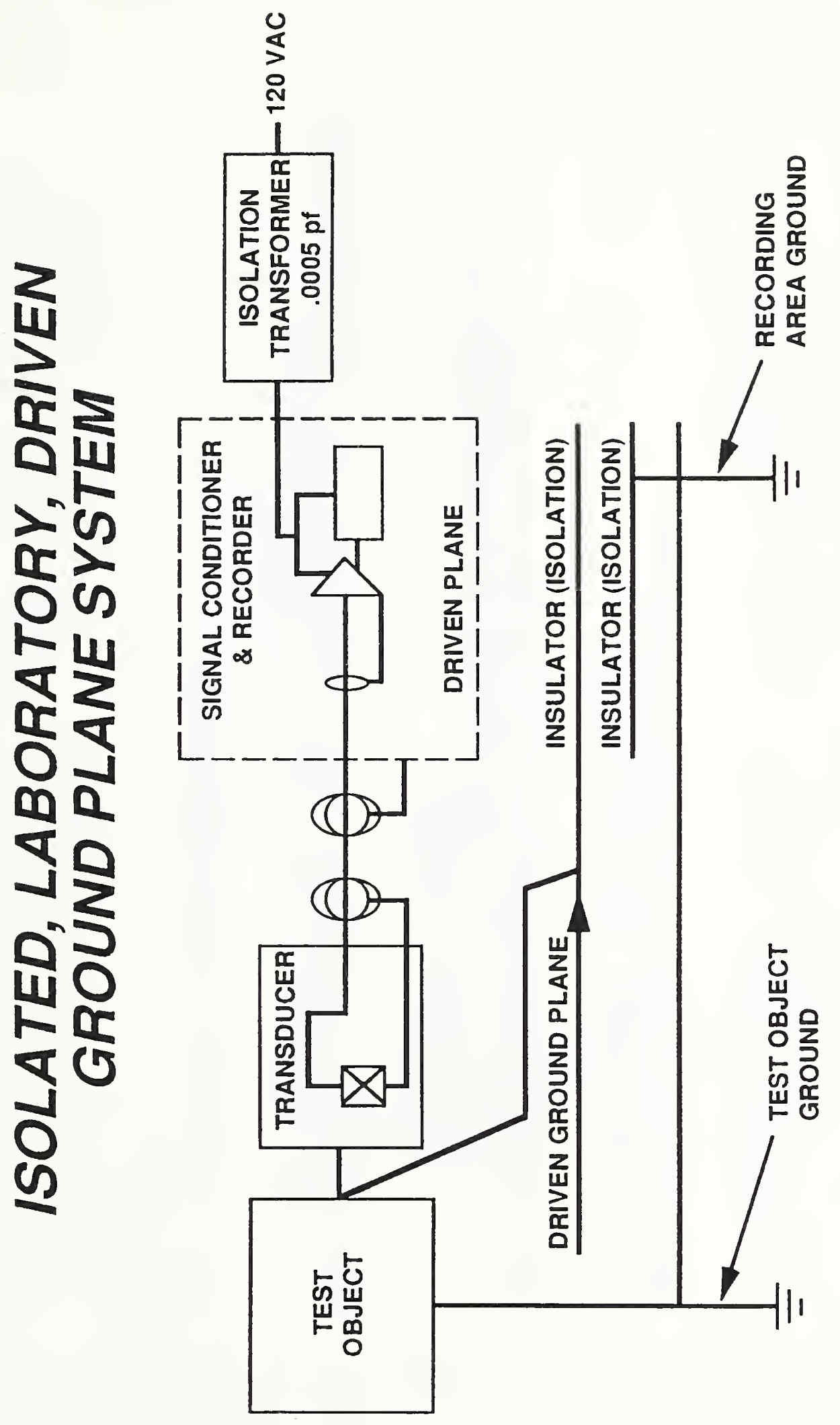




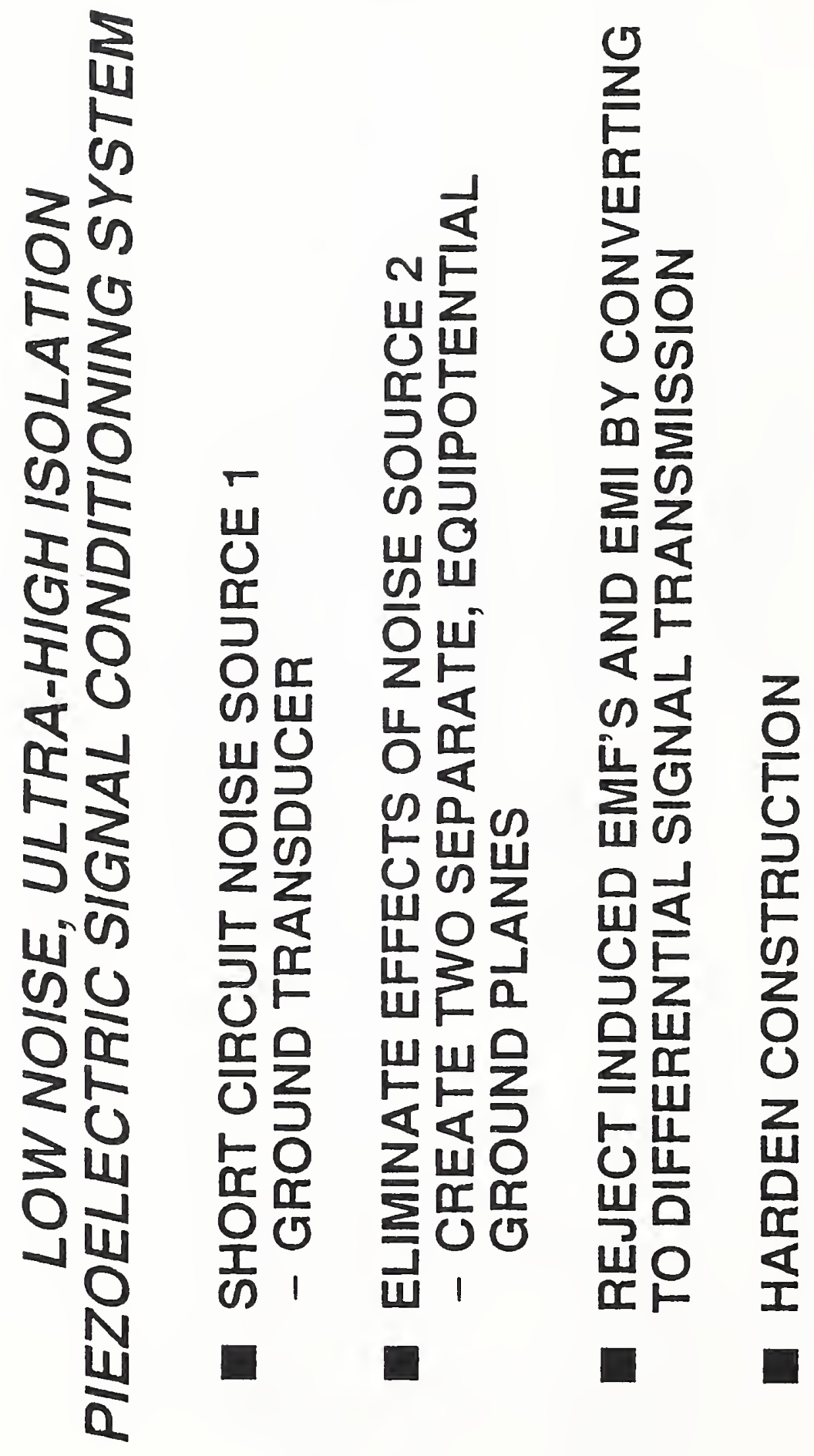




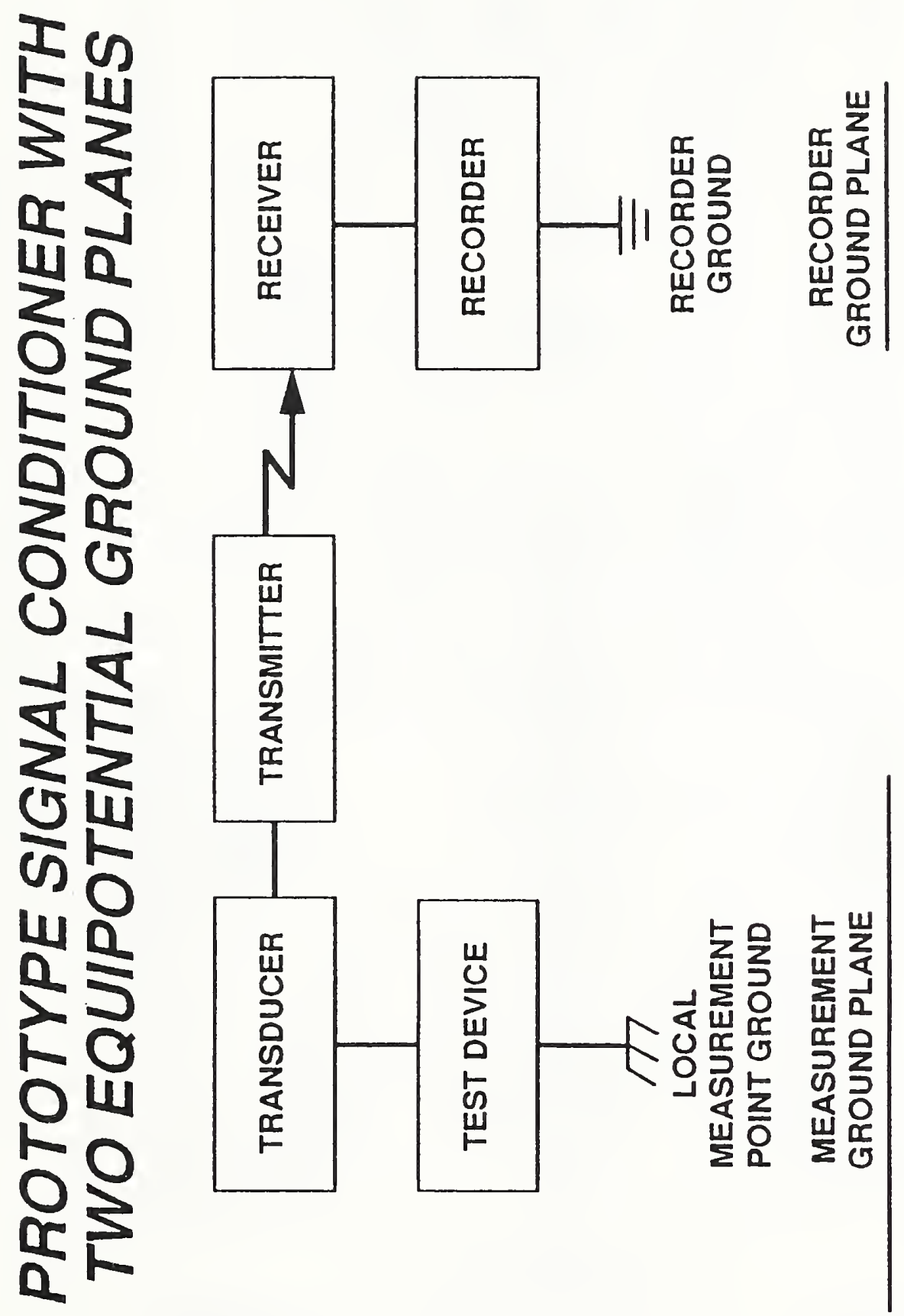




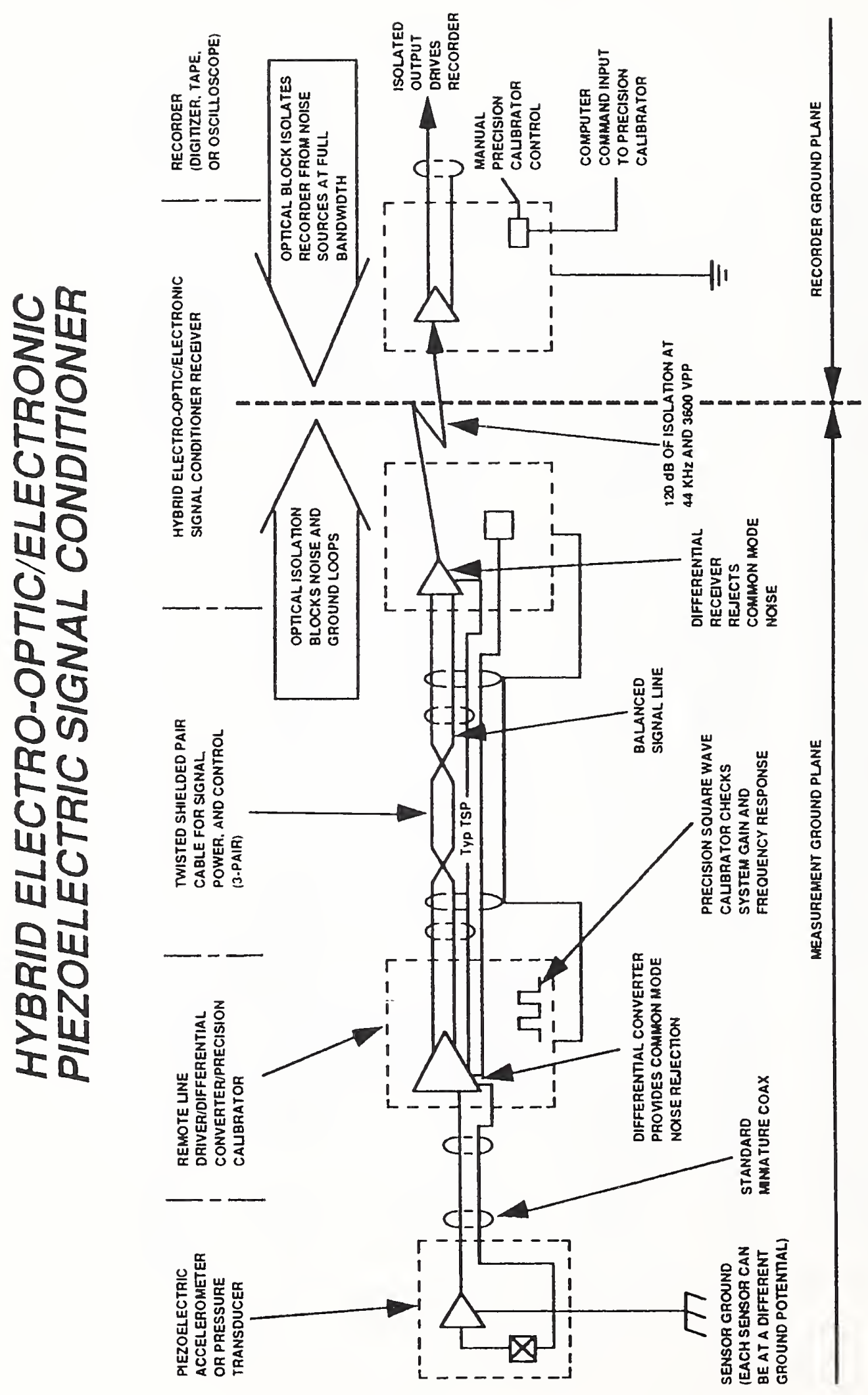




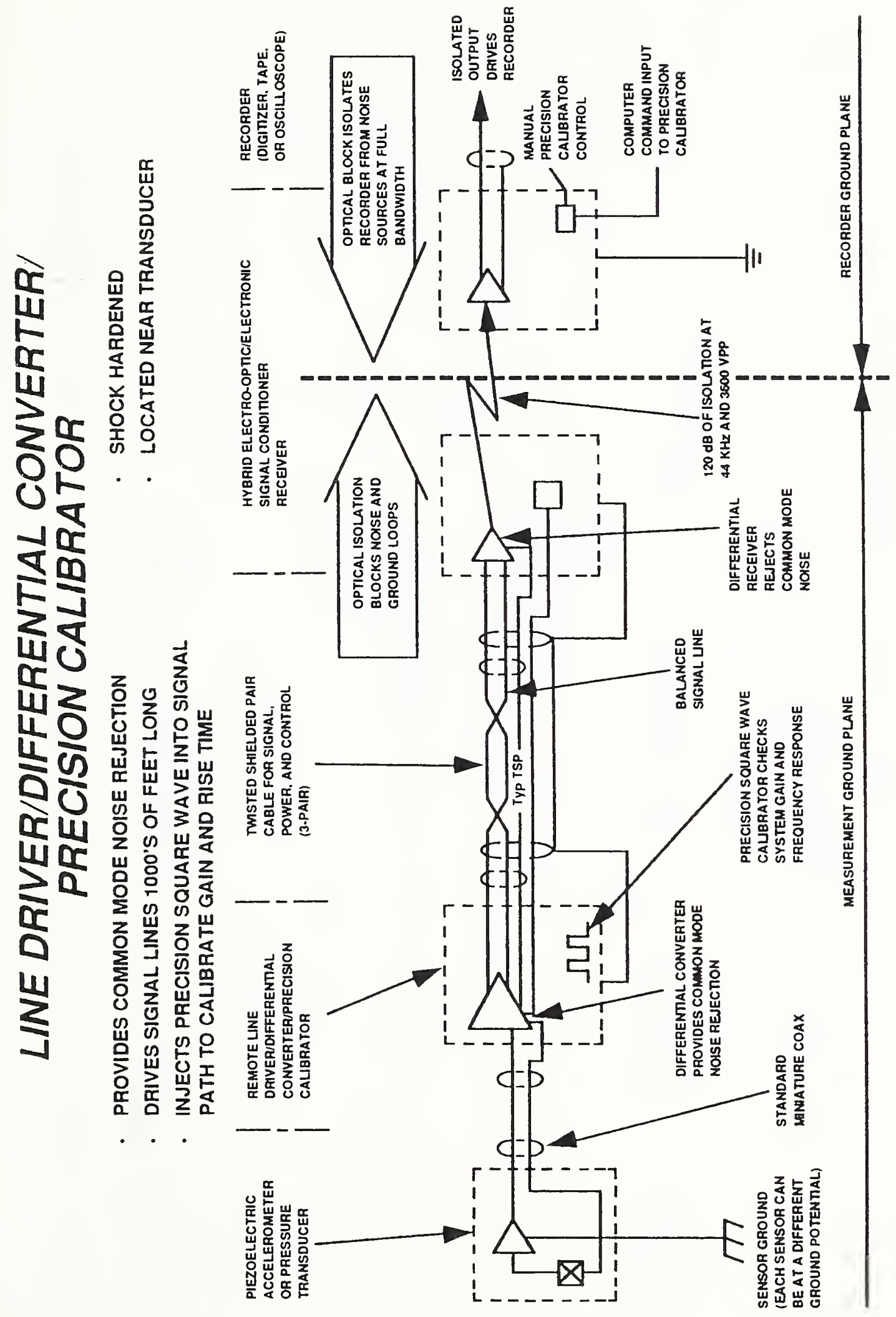




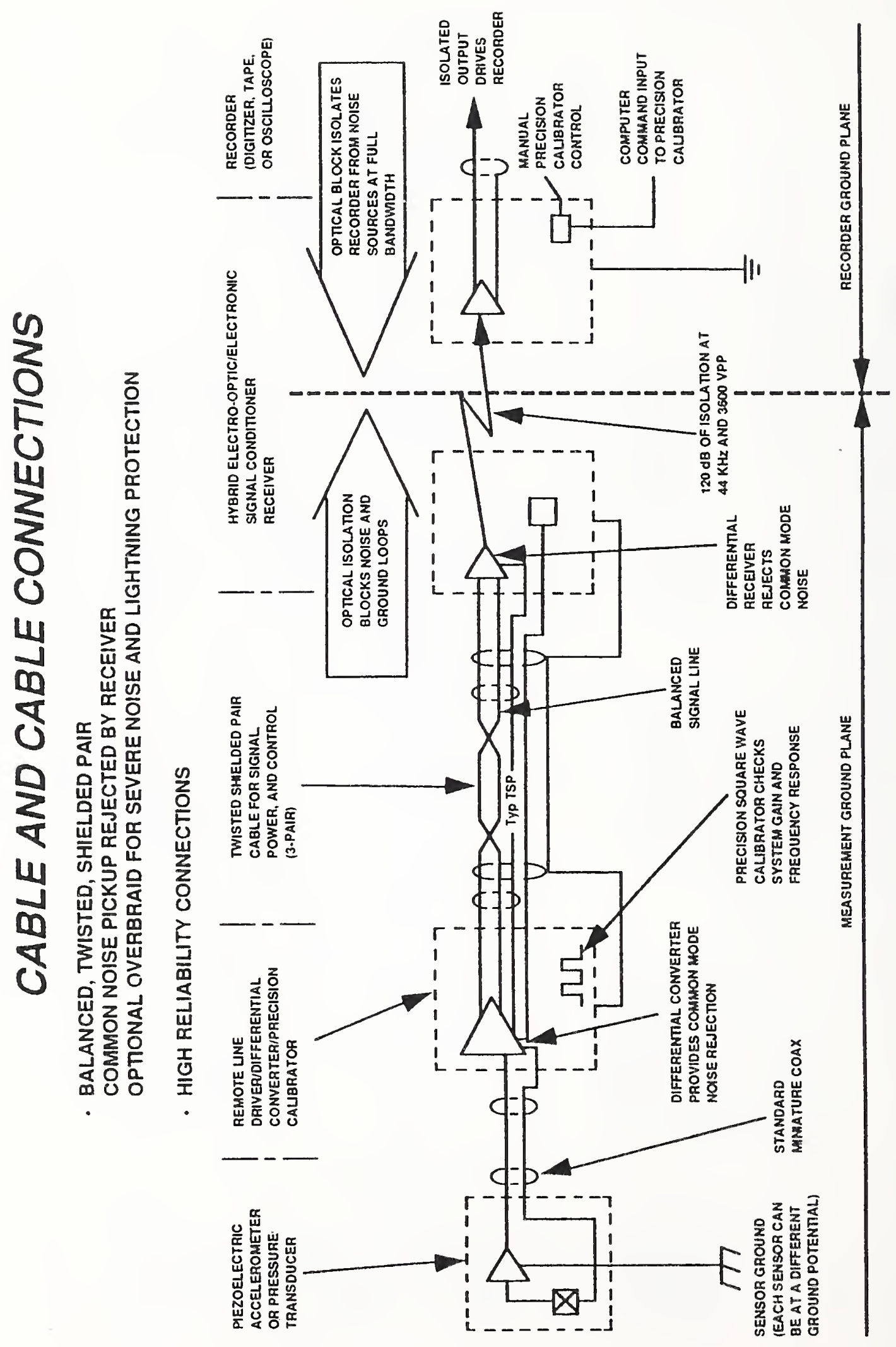




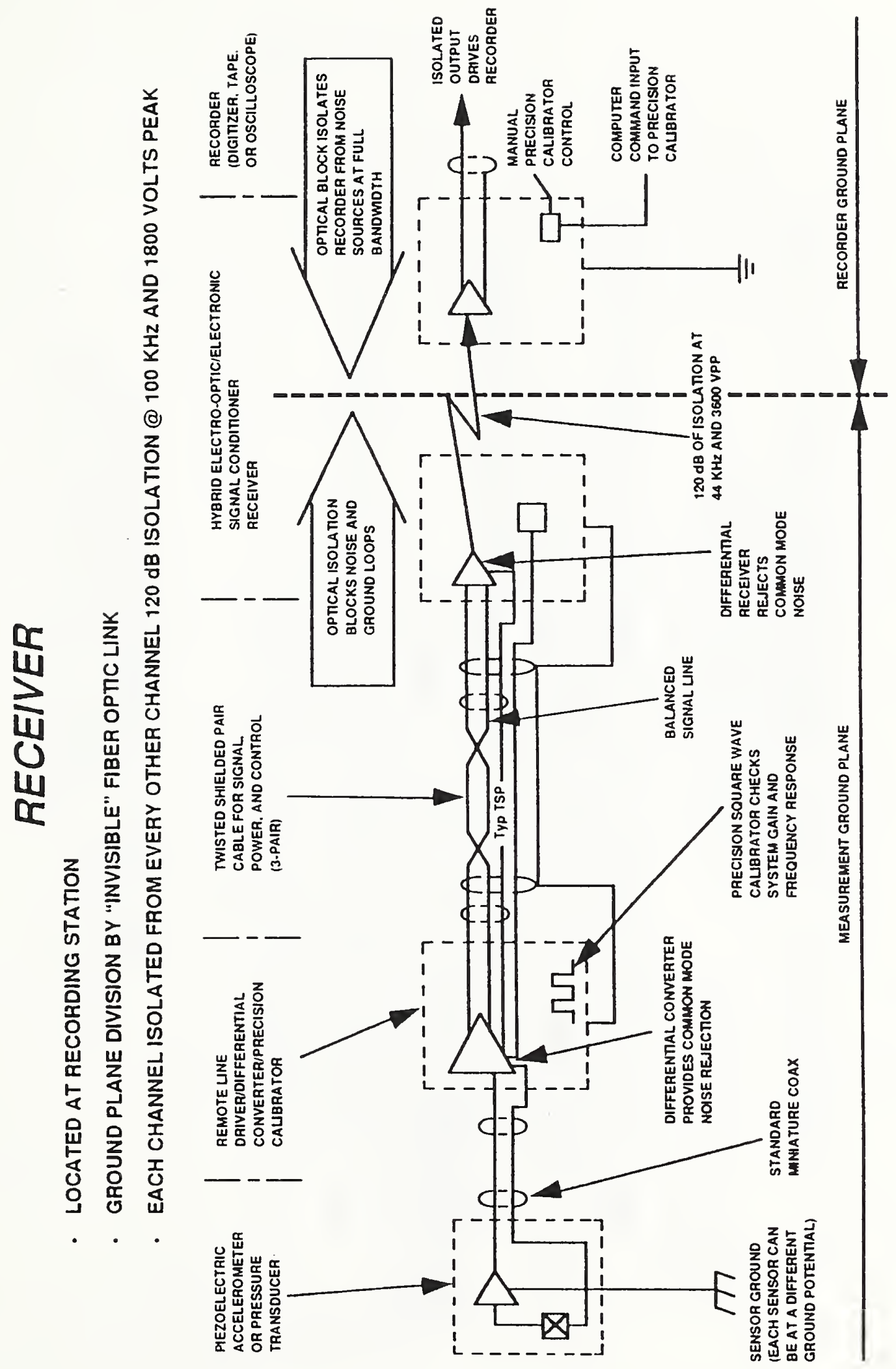




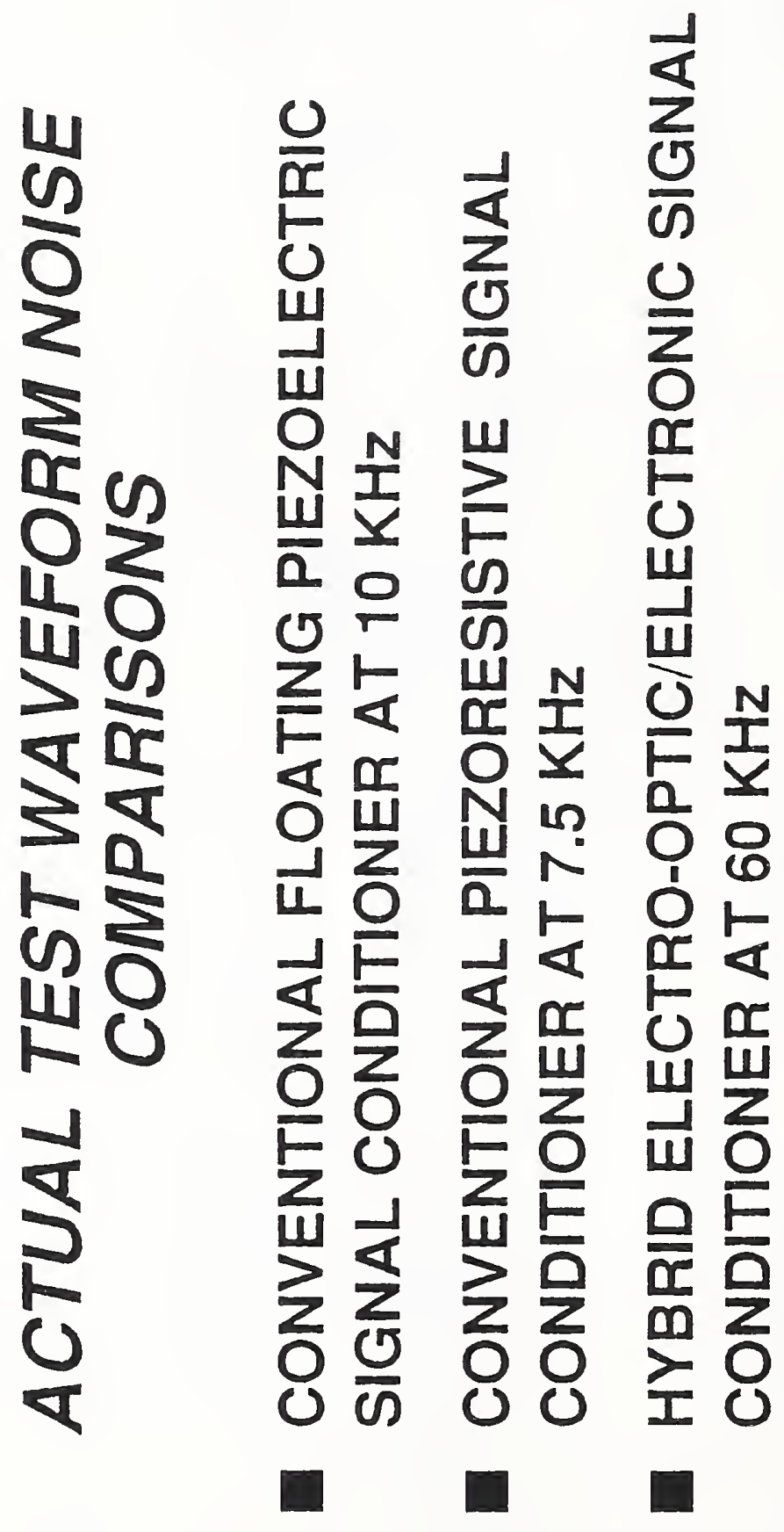




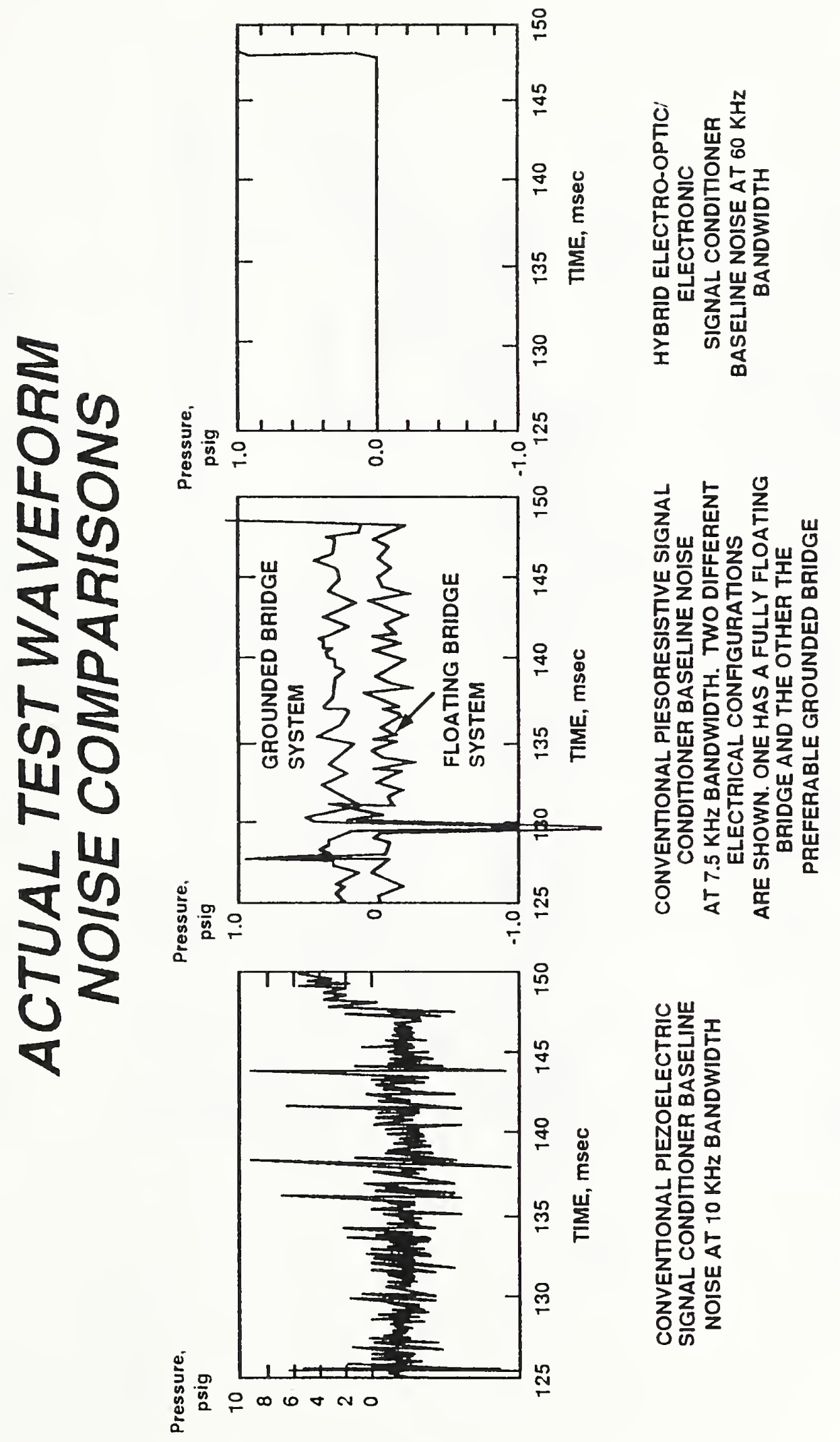




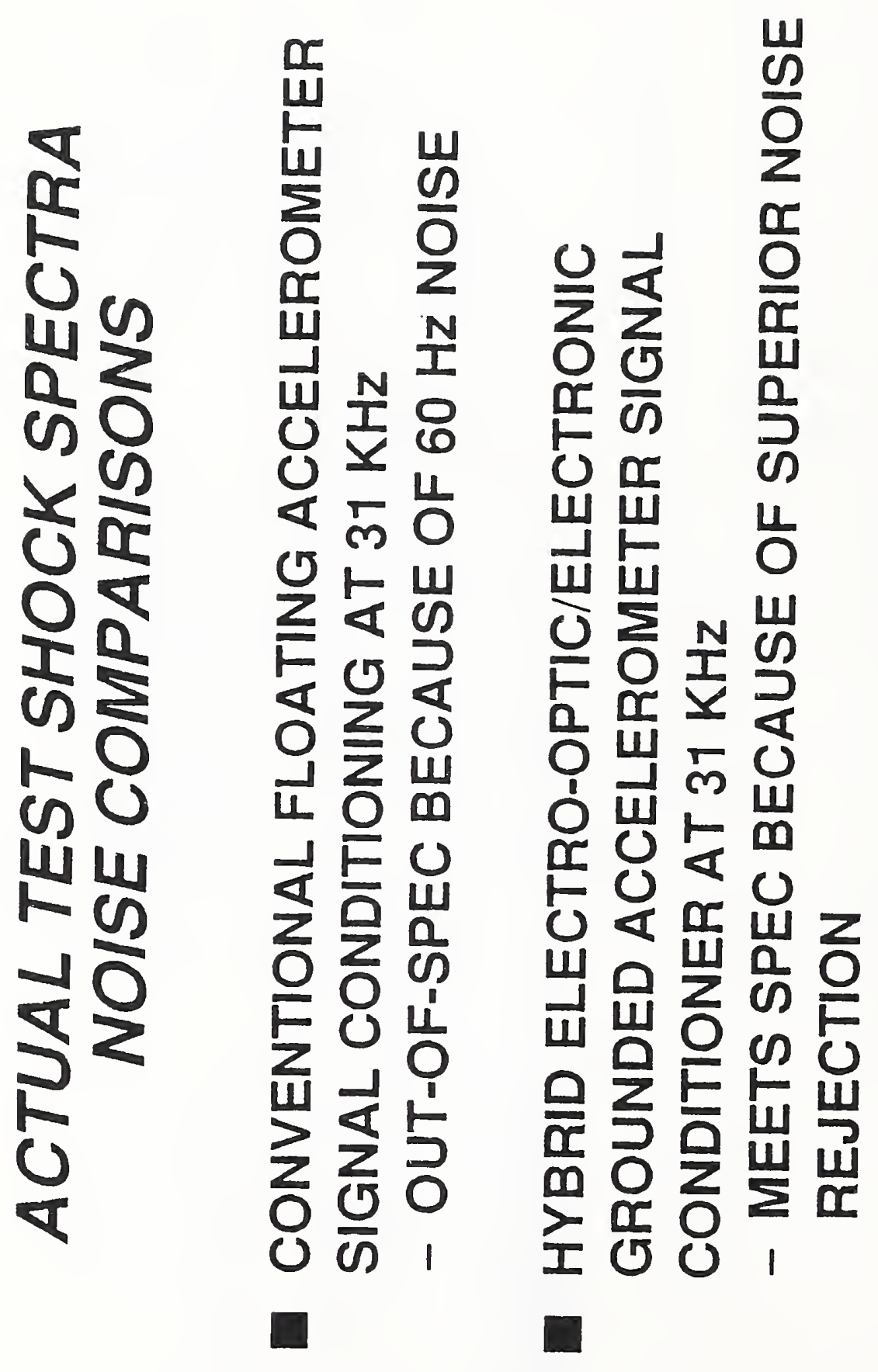




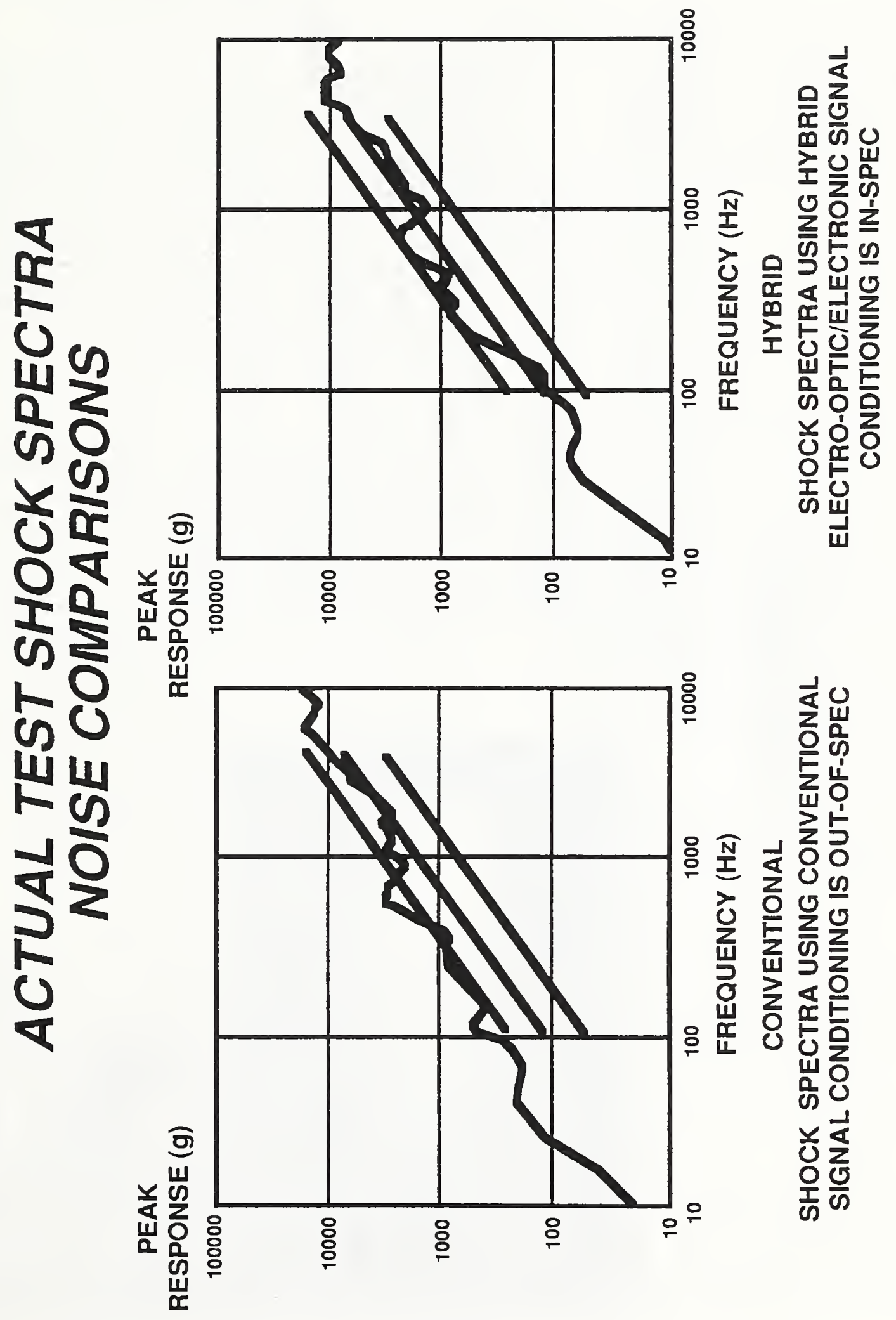




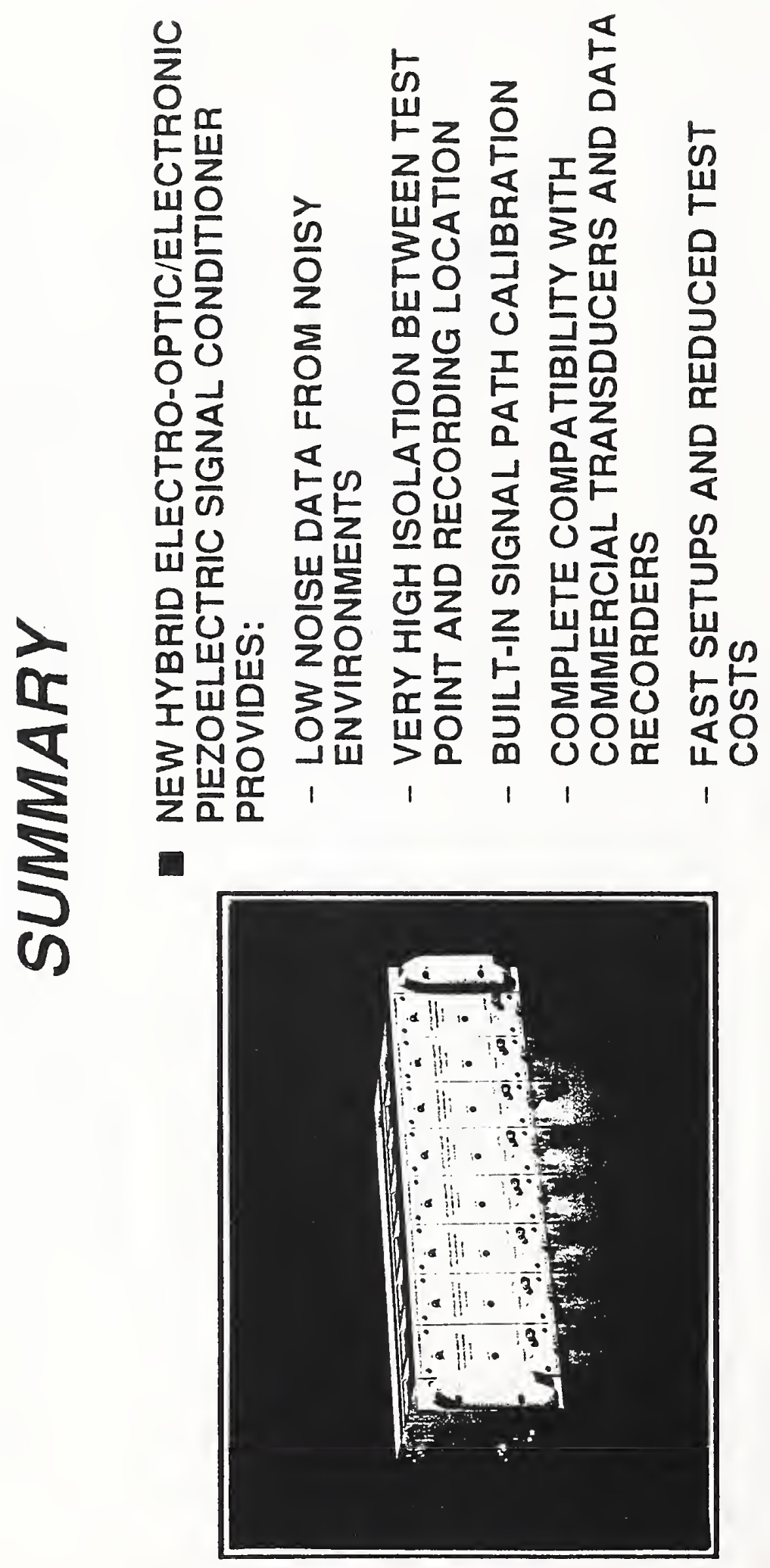


THREE CASE STUDIES OF STRAIN/TEST-INDUCED VOLTAGES WHICH OBSCURED SIGNALS

Peter K. Stein

Stein Engineering Services, Inc.

5602 East Monte Rosa

Phoenix, Arizona 85018-4646

Among the self-validation checks to which all data taken with impedance-based transducers must be subjected, is a documentation of the self-generating (voltage) responses from these impedance-based transducers. Metallic or semi-conductor strain gages and strain-gage-based transducers such as load cells, pressure transducers and accelerometers are known to generate voltages due to strain (or due to other test conditions). A simple way to document the presence or absence of these noise levels is to record a check channel which is not powered from an external source such as bridge supply voltage or current. It is also possible (but not in transient testing) to turn off such a supply during the test, on a single channel. The effect is DYNAMIC only not static

Three case studies have recently come to the author's attention and are described below.

Case Study 1: Charles P. Wright, Manager, Measurements Engineering Group. TRW $S$ \& T Div.

"A shock separation test was run at McuonnellDouglas in Huntington Beach, California in February 1990 . We had $1 / 8^{4}$ constantan quarter bridges mounted on the structure to check the survival of the installation technique under high shock loadings. The shock acceleration levels present at the gage locations was about 10,000 Gpk in the direction normal to the gage pattern. Levels in the other two directions were not measured.

"In one area we had duplicate gages mounted right next to each other - same gage, same direction, same installation, same technician, same materials, same everything. One was powered at 5 volts $D C$ in a Dynamics 7600A Signal Conditioners and recorded on analog tape $D C-75 \mathrm{KHz}$ bandwidth $(-3 d B)$. That is Fig. 10. The first 120 milliseconds of the record are shown in the top part... The lower left is the first $20 \mathrm{milli-}$ seconds to an expanded scale...

"Fig. 11 is the same data for the gage next door run with the excitation OFF. Beautiful, isn't it!" (WRIGHT 1990) TRW Space \& Technology Note that the strain-induced voltage noise is more than $10 \%$ of signal. Such noises cannot "be swept under the rug". As in the Pratt \& Whitney Case Study, they are the size of the rug!

Case Study 2: In 1987, Richard BILLIA was involved in a test on a $\frac{1}{2}$-scale model test vehicle in Sandia National Laboratory's Albuquerque $19 \mathrm{ft}$ diameter blast tube (see photo). Billia is with Lawrence Livermore National Labs. He reported (BILLIA 1987) the use of constantan CEA-062-UT-350 Measurements Group strain gages on a cylinder with the use of parallel gages again - one powered and one un-powered Figure 12 shows the two records. Test - induced voltages are again some $10 \%$ of signal.

Case Study 3: Rodney MAY of Sandia National Laboratory, Albuquerque reports in MAY (1991):

"Along with the Midgetman Program on which Sandia was working, the 19-ft diameter blast tube was being expanded. Before the full test, one with a 4-charge was run. Strain gages were put on the blast tube to see if the stress calculations had been correct.

"They were getting near $75 \%$ of yield stress and were worried that the tube would fail at full 1 charge. I asked them if they had run any noise-check gages. Their reply was a classic: 'No, no ! We needed all the channels available to get our data!"

"A couple of days later we put 2 check channels on: one un-powered gage mounted to the tube, and one un-powered and un-strained, on a 'dummy' block. That way we could observe the straininduced voltages and any antenna effects. After the shot they showed me the results, but only briefly, and documentation was ever issued. It turned out that $80 \%$ of the signal they had obtained were due to antenna effects, which the un-powered, un-strained gage documented very nicely.

"Noise-check channels do give you information, but you have to do battle for them!"

PHOTO: SANDIA NATIONAL LABORATORY'S ALBUQUERQUE $19 \mathrm{FT}$

DIAMETER BLAST/SHOCK TUBE. An explosive charge detonated at the far end of the shock tube produces a shaped blast wave used in testing responses of a structure under such a wave.

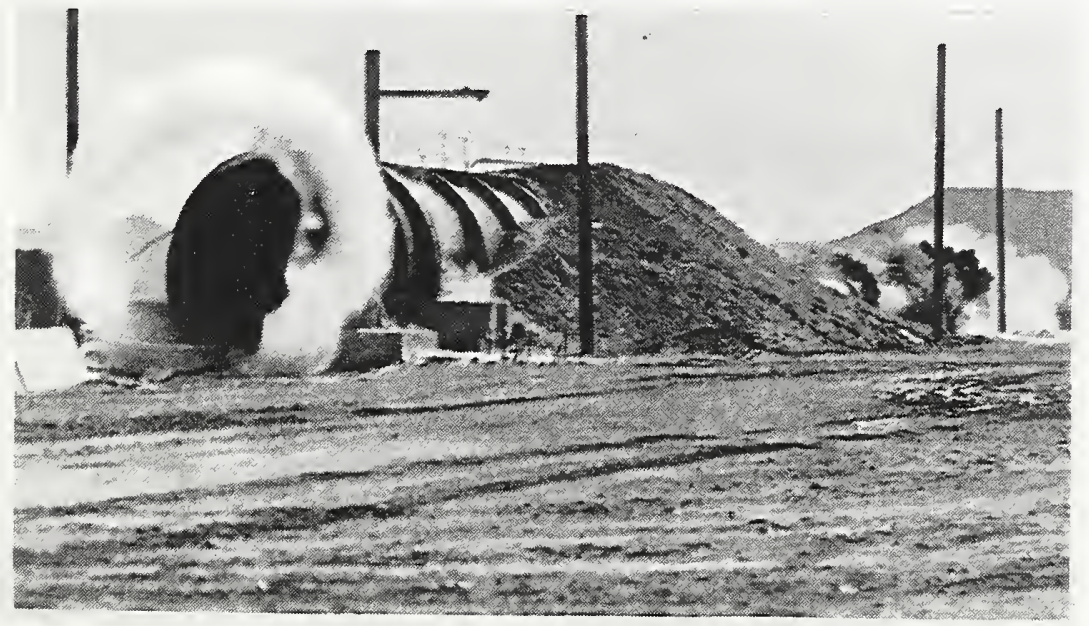

\section{REFERENCES:}

All 3 case studies are reported in: Proc. Western Regional Strain Gage Committee Meeting, February 5-6, 1991 , Tempe, AZ. From: 42 N. Benson Ave, Upland, CA 91786.

WRIGHT (1990): Letter of March 21 to Peter Stein - also reporting Case Study 1.

The cause for these strain-induced voltages is not as yet understood but has been found in all metals, especially strain gages, Nickel (as in leads and resistance thermometers, and in Alumel, as in thermocouples. The author has collected many references to this phenomenon. 

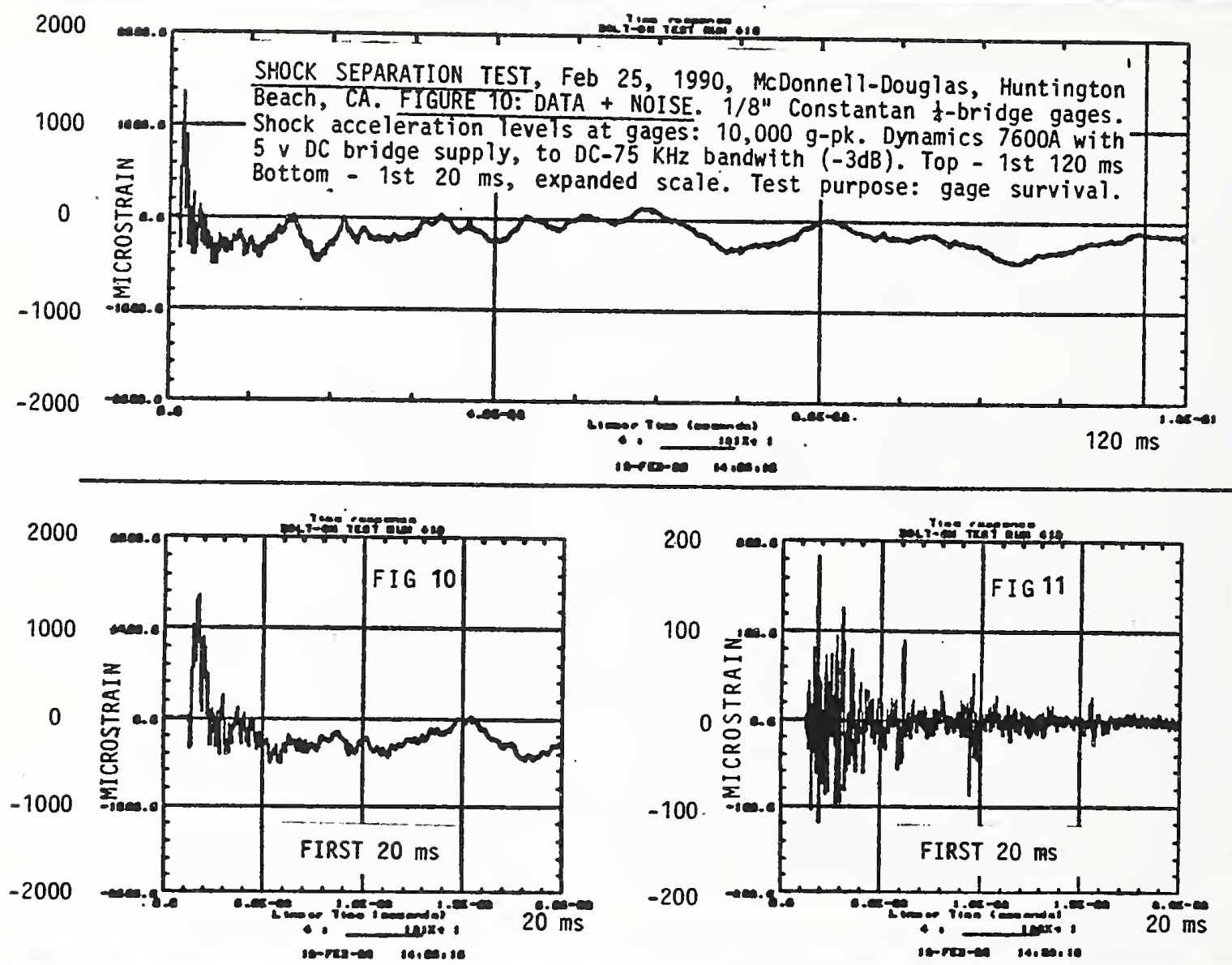

SDFC I-IEAS 4.0: Test Data Pnalysis

25-FEB-9B

B8:58:30

FTREABE: ENLT-IN! FIFUTEEHWL SH:LI: TESTS

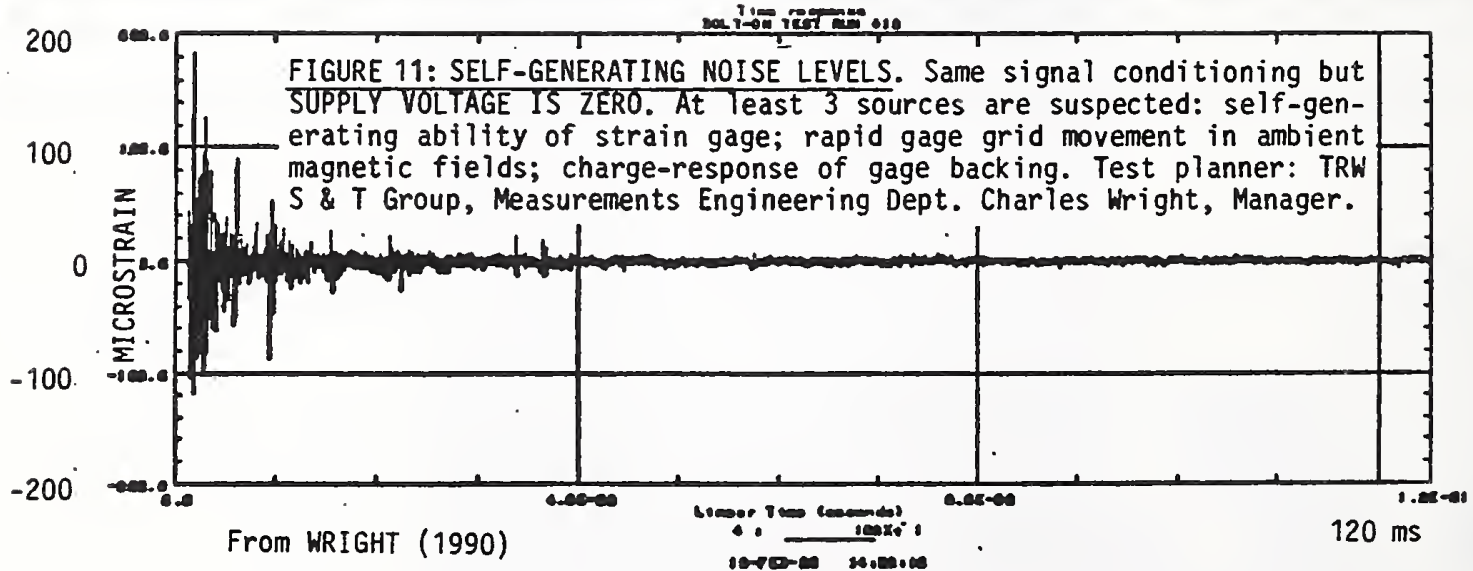




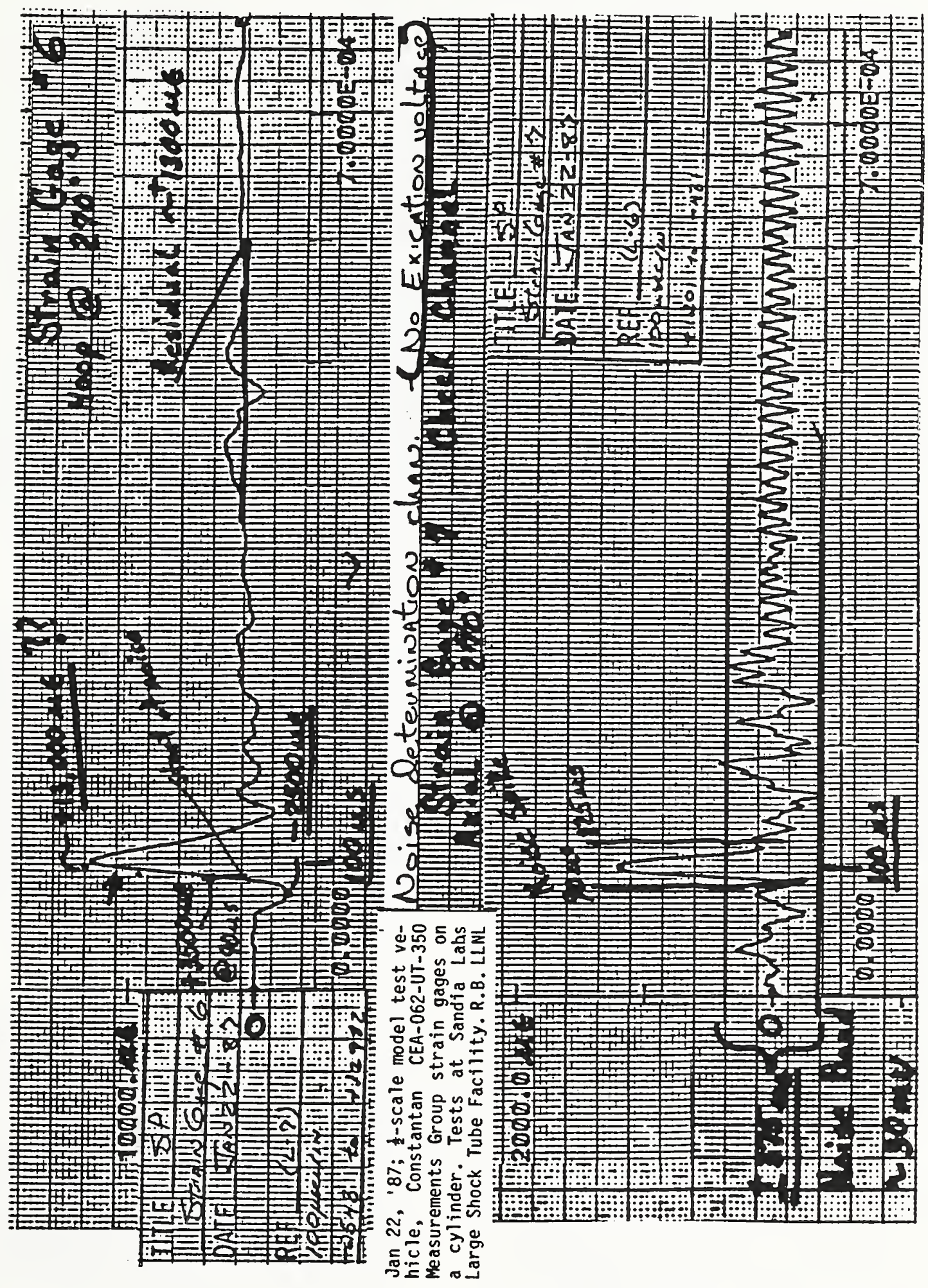

From BILLIA (1987) 


\section{CALIBRATION OF BALLISTIC PRESSURE TRANSDUCERS}

\section{ARPAD A.JUHASZ, CHARLES D. BULLOCK AND DONALD H. NEWHALL}

Techniques for calibrating ballistic pressure transducers include the ballistic pulse method, shock-tube methods and pressure-step techniques. A positivegoing, high-pressure-step generator is useful in calibration, servicing and development of dynamic high-pressure transducers. A positive-going step calibration device allows accurate, safe and simple dynamic calibration and evaluation of ballistic pressure gages. The gage response obtained can be related to its static (deadweight) behavior. Several gages may be evaluated simultaneous$l y$, relative to a common dynamic event. The calibrator may also be used as a diagnostic tool in analyzing and developing experimental pressure gages.

Athough ballistic pressure transducers are used to measure events which occur in milliseconds, the determination of their response characteristics has been limited to static calibration against a deadweight pressure standard. Although the DWT is a primary standard, the assumption is made that the static and dynamic responses of the gage in question are equivalent. Obviously, any differences in gage response to static and dynamic events can lead to serious measurement errors. There has been a general agreement that dynamic techniques are needed to supplement current static calibration methods. Several techniques developed to address this problem are discussed ${ }^{1,2}$ -(1) the ballistic pulse method, (2) shock-tube methods, (3) negative-going pressure step and (4) positive-going pressure step,

\section{BALLISTIC.PULSE METHOD}

In one version of the ballistic-pulse technique, the gage is mounted at the end of a tube in contact with a hydraulic nuid confined by a movable piston. The tube guides a projectile which impacts the piston to create a pressure pulse in the fluid. Different pressures may be achieved by varying the compressibility of the fluid, the mass of the piston, and the mass and velocity of the projectile. From these variables the reference pressure may be calculated.

The pulses rise within milliseconds, and mimic the characteristic rising and falling of a ballistic presaure pulse.
One such device, capable of operating to a pressure of $100,000 \mathrm{psi}$, is operational at the Combat Systems Testing Activity (CSTA), Aberdeen Proving Ground, Maryland. ${ }^{3}$ This method is useful only for dynamic comparison of different pressure gages because variations in projectile velocity, frictional effects on the moving piston, and other energy losses make it difficult to accurately compute the actual delivered pressures. Because a projectile is fired during the calibration process, this method requires extensive safety provisions, not readily available in most laboratories.

\section{SHOCK-TUBE METHODS}

There are two general approaches to shocktube calibration. In the first, the test gage is mounted in the end wall of a tube and subjected to a reflecting shock wave. The gage output is monitored as the shock front arrives at and reflects from the end wall.

In the second approach, the gage is mounted in the side wall of the tube and its output is monitored as the shock front passes. Buth methods generate rapidly rising pressure pulses that are readily calculated by thermodynamic principles using velocity measurements and known gas properties. Shock-tube methods are useful in establishing the dynamic respunse characteristics of pressure gages. However, calibration is generally limited to pressures below $1000 \mathrm{psi}$, whereas ballistic applications require much higher pressures. 


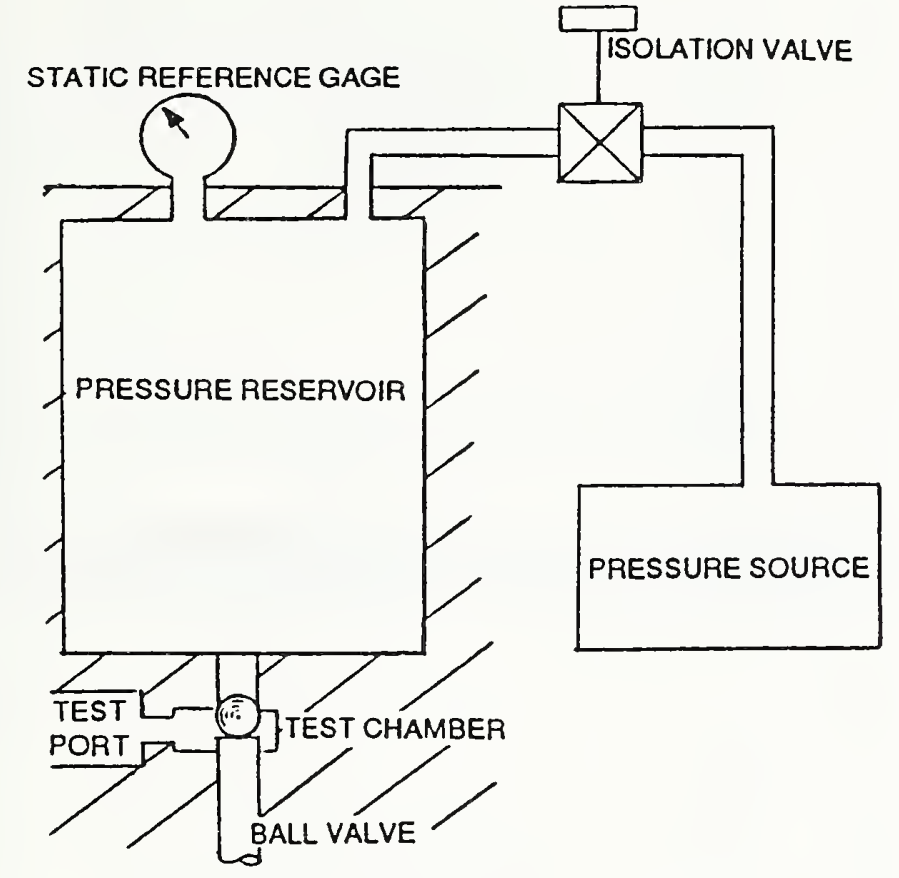

FIGURE 1. Positive-pressure step technique.

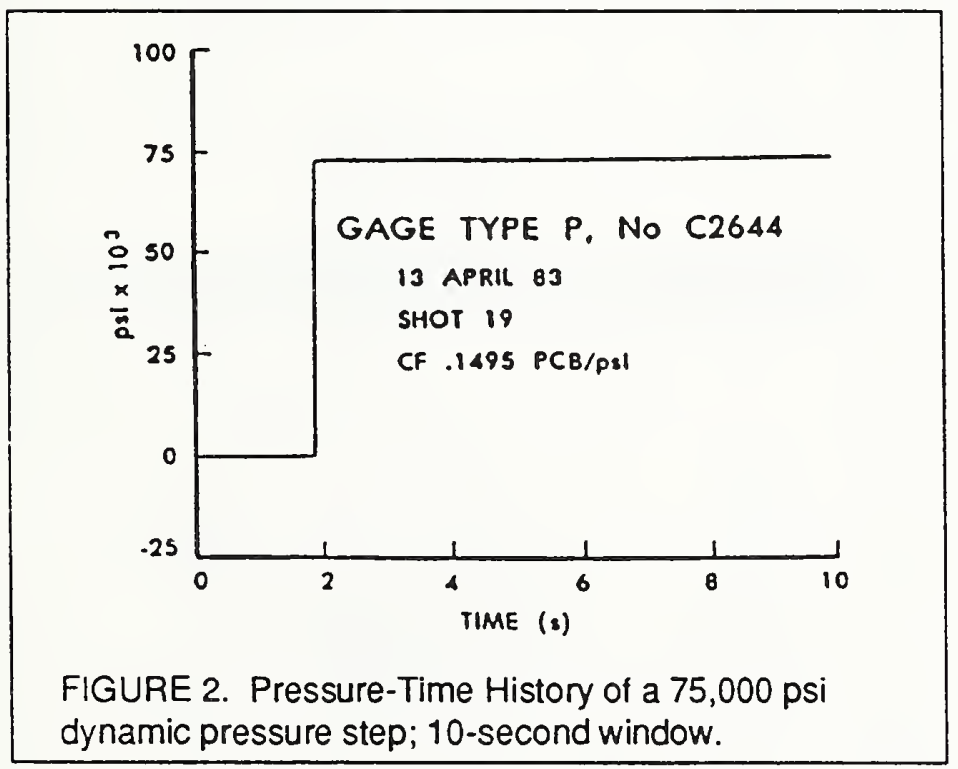

NEGATIVE-GOING PRESSURE-STEP METHOD

In this technique, the gage is exposed to a high pressure under static conditions, using a hydraulic fluid. The pressure on the gage is then relieved using a fast-acting dump valve, bringing the system to atmospheric pressure. The response of the gage output obtained during the depressurization is assumed to be the inverse of the corresponding positive pressure step.

This method's strengths include its relative simplicity and suitability to use in calibration facilities, and quick response, typically 100 microseconds or less. However, the major assumption, that the positive response of the gage is equal and opposite to the negative response of the gage, is not completely accurate; pressure preloading of the gage-tomount interface and hysteresis can cause significant differences between the magnitudes of the pressurization and depressurization pulses.

\section{POSITIVE-PRESSURE-STEP METHOD}

Figure 1 illustrates the basic configuration of the positive-pressure-step device. A large pressure reservoir is connected to a much smaller test chamber by a fast-acting ball valve. The test chamber is equipped with several gage ports and a vacuum port which aids in filling the reservoir and setting the baseline pressure in the test chamber. Reservoir pressure is provided by a (Harwood) hydraulic high-pressure-generating panel. The pressure is monitored by a static reference gage whose calibration is traceable to NIST.

The large ratio of reservoir to test-ch amber volume minimizes the overall system pressure drop while generating the pressure step, thereby reducing stress loading on the system components (particularly the static reference gage). The maximum operating design pressure is $150,000 \mathrm{psi}$, and the action time (10$90 \%$ of peak pressure) is well under one millisecond. Outputs of both the static reference gage and the ballistic test gages are monitored during the test. The final steady-state output of the reference gage is taken as the true value of the pressure-step maximum. Short- and long-term monitoring of the test gage outputs establish a relationship between dynamic and steady-state responsive behavior.

\section{PERFORMANCE ANALYSIS}

Examples of both short- and long-term responses of a commercial piezoelectric pressure gage to a positive-going pressure step are presented in Figures 2 through 5. Figure 2 shows a typical pressure versus time history for a 75,000 psi pulse acquired over 10 seconds. Monitoring the test gage response for $10 \mathrm{sec}-$ onds allows correlation to the steady-state response of the static reference gage. Figure 3 shows a 20-millisecond window of the same 75,000 psi pulse; Figures 4 and 5 present progressively shorter windows. 
Overlaying the traces indicates that the steady-state value of the pressure step is rapidly achieved and held, after initial oscillations have died out. Similar oscillations have been reported in lower-pressure devices by both Smith and Dykstra ${ }^{4,5}$; one may conclude that these oscillations are caused by actions within the pressure-generating system and the mount, not by the gage itself.

One important application of the step calibrator is comparing the responses of different types of pressure gages to the same input. Figures 6 and 7 show pressure versus time histories of both a piezoelectric gage and a strain-gage measuring a positive-going 100,000 psi pressure step. The traces are virtually identical, exhibiting initial system oscillations which decay quickly.

The calibrator may also be used to analyze the behavior of experimental gages by comparing their output with a known standard. Figure $\mathbf{8}$ shows pressure versus time history of a developmental pressure gage subjected to a 125,000 psi pressure pulse. This curve exhibits, as does the known standard, a smooth pressure rise and stable output after the peak pressure has been attained.

The positive-step pressure generator, in conjunction with conventional deadweight calibration methods, can be useful in tracing dynamic gage-response problems. Figure 9 exhibits the pressure history of a developmental pressure gage exposed to a 130,000 psi pressure step. The trace indicates a clear upward drift after the step is complete. The same gage had exhibited good response behavior on static calibration using the deadweight system. In gun tests, however, the device read $5 \%$ low. From the dynamic calibration tests, it became clear that it took tens of milliseconds for the gage output to reach a maximum. This could not be detected by a static calibration technique. On dissecting the gage, it was found that the bond between the strain patch and the gage body was faulty. A defect of this type could account for the differences in static versus dynamic behavior noted in testing.

The equipment and method of dynamic high-pressure pulse generation for calibration and test purposes has the following advantages:

1. Direct on-site calibration traceable to NIST. Noinvolved calibration calculation models are required.

2. Calibration is not affected by changes in testchamber volume.

3. The equipment does not require special foundation provisions.

4. The equipment does not require special alignment during installation.

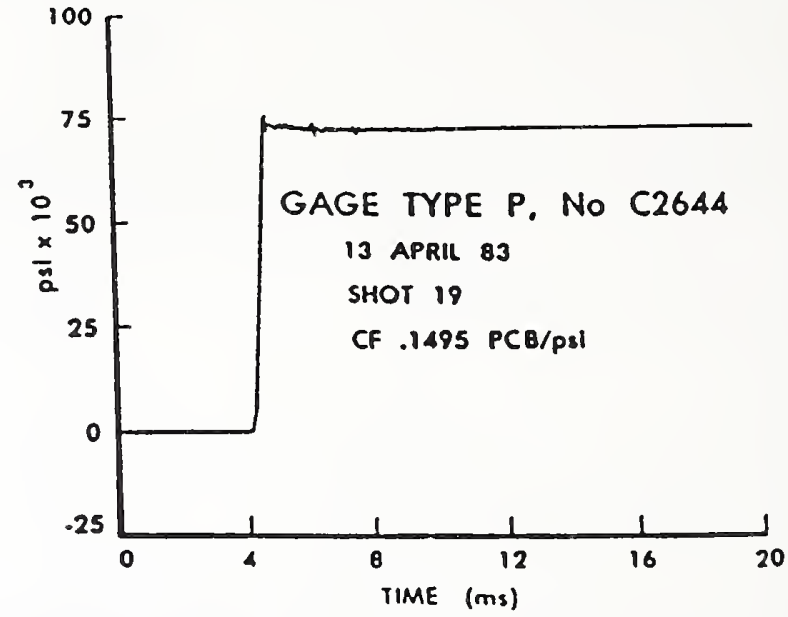

FIGURE 3. Pressure-Time History of a 75,000 psi dynamic pressure step; 20 -millisecond window.

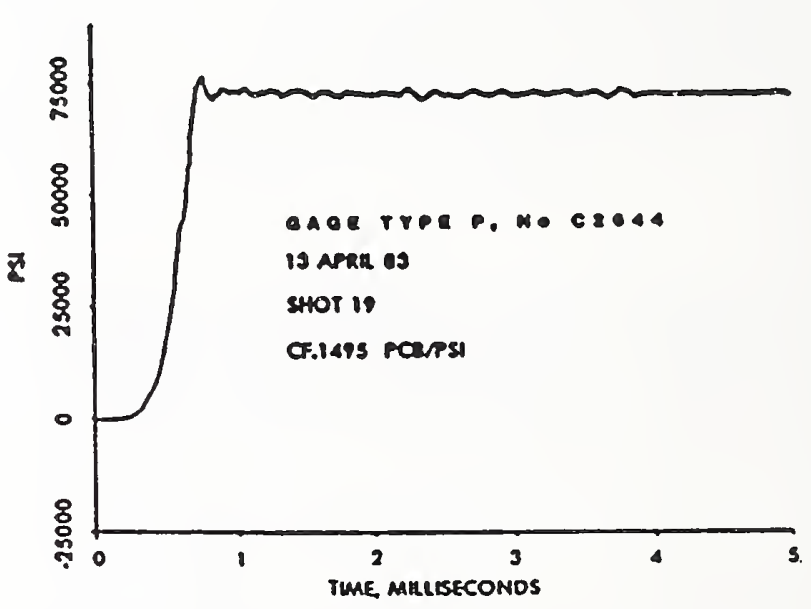

FIGURE 4. Pressure-Time History of a 75,000 psi dynamic pressure step; 5-millisecond window.

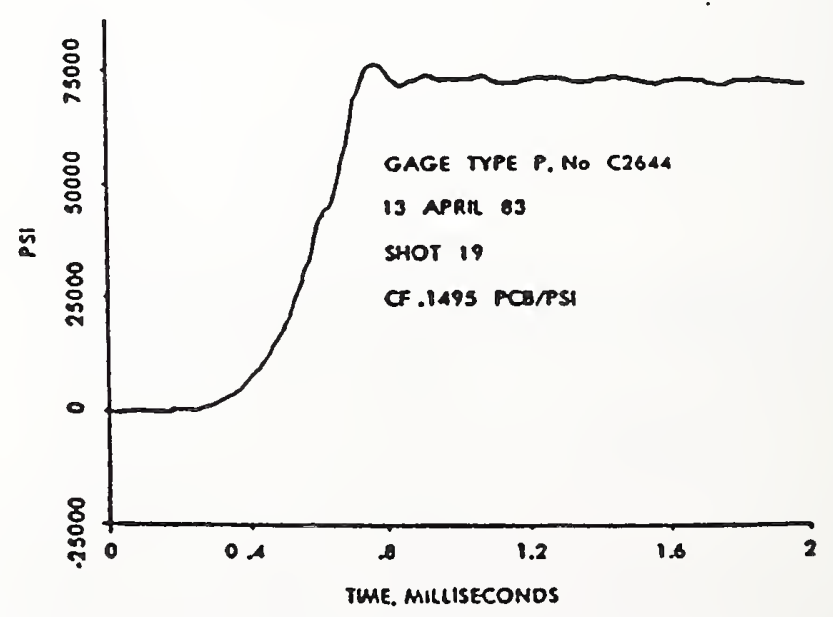

FIGURE 5. Pressure-Time History of a 75,000 psi dynamic pressure step; 2-millisecond window. 


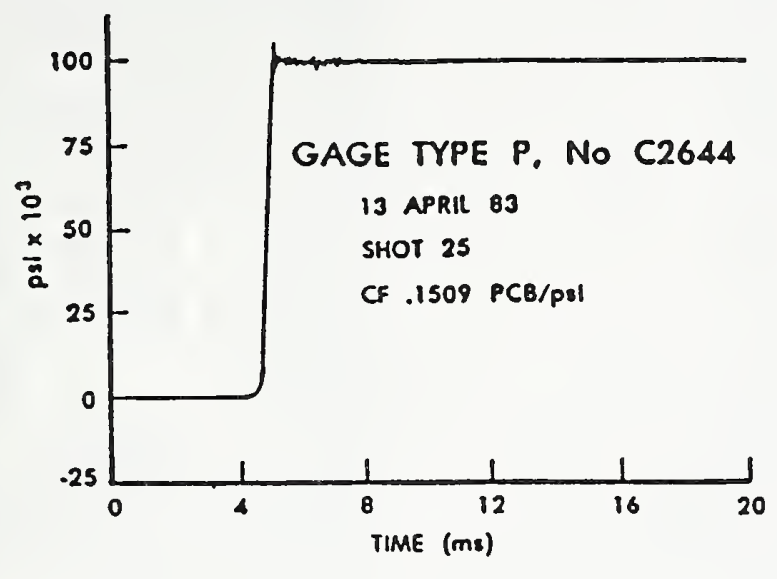

FIGURE 6. Response of a Piezoelectric Ballistic Pressure Gage to a 100,000 psi positive pressure step.

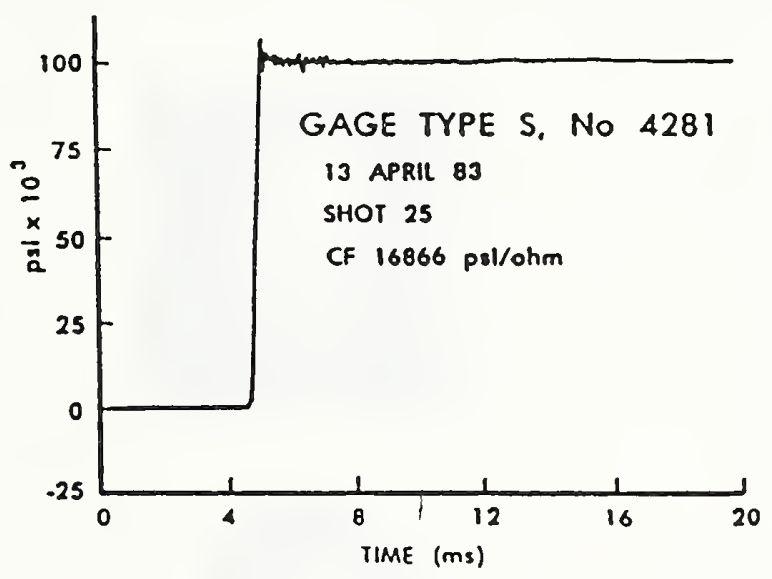

FIGURE 7. Response of a Strain-Type Ballistic Pressure Gage to a 100,000 psi positive pressure step.

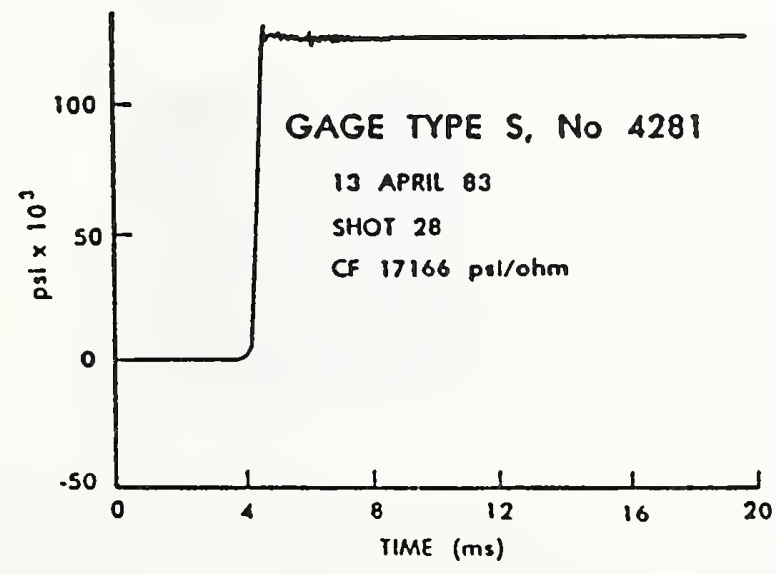

FIGURE 8. Response of a Developmental Pressure Gage to a 125,000 psi positive pressure step.

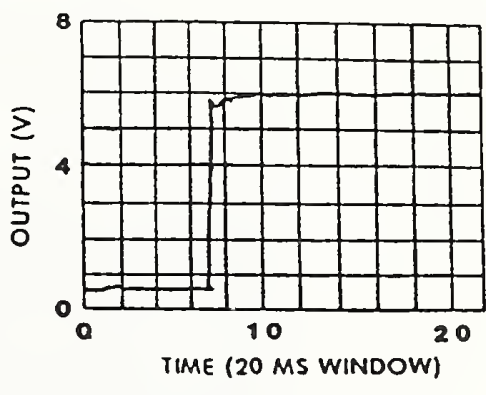

FIGURE 9. Response of a Faulty Developmental Pressure Gage to a 130,000 psi positive pressure step.

4. The equipment does not require special alignment during installation.

5. Mechanical parts, critical to accurate calibration, are not subjected to severe impact shock or stress.

6. Operation does not involve expendable parts or supplies.

7. Peak pressure can be maintained to monitor the final steady-state output of the test gages.

8. A negative-going pressure pulse is available as an option.

The design parameters of the equipment can be varied easily so that greater accuracies and still higher pressures can be attained. The device can be turned to knotty problems in internal ballistics as they emerge. Similar equipment could be useful in fundamental studies in physics, such as thermodynamics, reaction and diffusion rates, and physical properties of materials under dynamic pressure loading. The speed of compression loading is fast enough to approach isentropic compressions.]

\section{REFERENCES}

1. NBS (NIST) Report 4440, "Measurement of High Pressure: Bibliography, Index and Preliminary Survey", W.G. Brombacher, U.S. Dept. of Commerce, NBS, October, 1955.

2. NBS Technical Note $914,{ }^{\circ A}$ New Dynamic Pressure Source for the Calibration of Pressure Trans. ducers," Carol F. Vezzetti, John S. Hilton, J. Franklin Mayo-Wells and Paul S. Lederer, U.S. Dept. of Commerce, NBS, June 1976 (and references therein).

3. Personal communication with J.D. Dykstra of Combat Systems Testing Activity, Aberdeen Proving Ground, Maryland.

4. Smith, R.O., "A Liquid Medium Step Function Pressure Calibrator," ASME Paper \#63-WA-263, ASME, 345 E. 47th Str., NY, NY.

5. Dykstra, J.D., "Evaluation of Pressure Transducer Response with a Pressure Step Generator," Bulletin of the 11th Meeting of the Joint Army-Navy Force Solid Propellant Rocket Static Test Panel, SPIA Publication SPSTP/ 11, September, 1962.

6. Bullock, Charles, and Juhasz, Arpad A., "Ballistic Pressure Transducers," Measurements \& Control, April 1990. 
FIGURE 10. Dynamic Positive Step Pressure Generator. Trunion mounted pressure vessel assembly with pressure generation control panel.

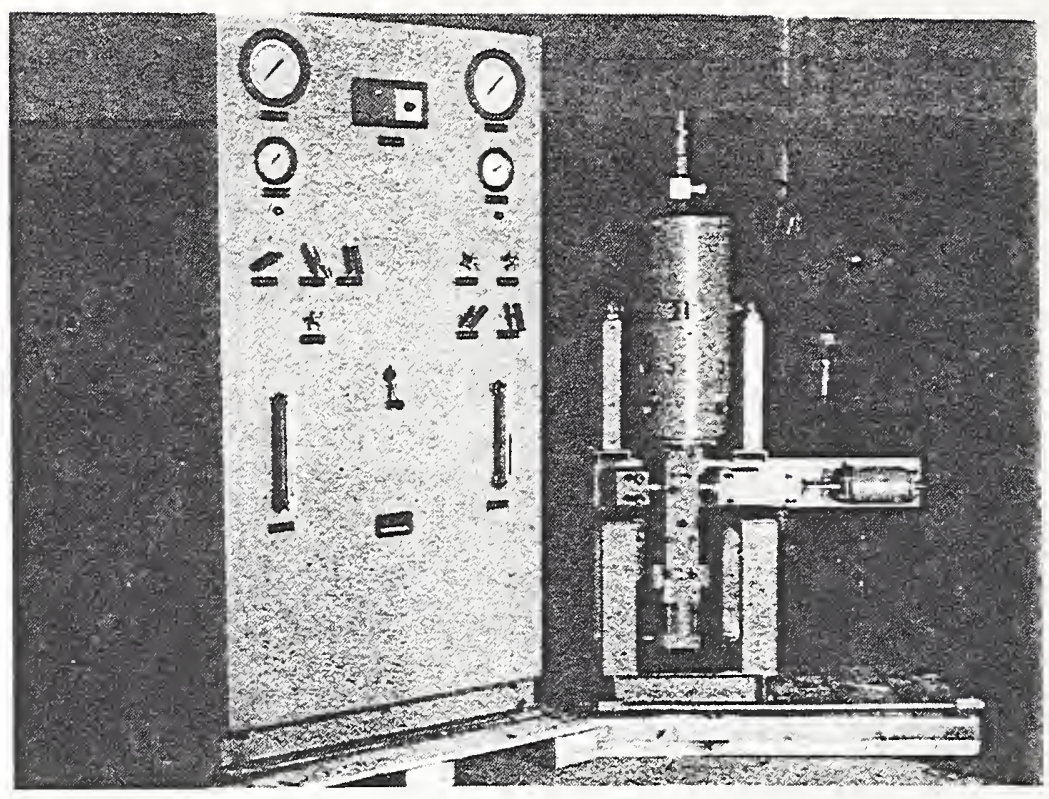

\section{THE AUTHORS}

CHARLES D. BULLOCK is a Senior Mechanical Engineering Technician at the U.S. Army Ballistic Research Laboratory, Aberdeen Proving Ground, MD. He has over 20 years experience in ballistic pressure gage calibrations. Mr. Bullock contributed to the development of static and dynamic pressure calibration methodology, the development of ballistic pressure transducers for measurements to 200,000 psi, and to the development of a laboratory 100,000 lb force standard.

ARPAD A. JUHASZ is a Team Leader at the Ballistic Research Laboratory. He has over 20 years experience in high-pressure combustion studies, related pressure trans. ducer development and calibration methods, and research in advanced gun propulsion technologies.

DONALD H. NEWHALL is principal owner and founder of Harwòd Engineering Co., Inc., designers and manufacturers of high-pressure industrial and laboratory equipment. His background in this field includes a WWII stint in Army Ordnance at Watertown Arsenal as officer in charge of high-pressure design, testing, production cuto-frettage and related $R \& D$ efforts. Directly postwar he was in charge of highpressure efforts in $R \& D$ at the Foxboro Com. pany, prior to founding Harwood Engineering in 1949. Throughout his career he has maintcined an interest in pressure and force standards. He introduced many innovative designs that have been patented, and has published many articles in the field of extreme pressure.
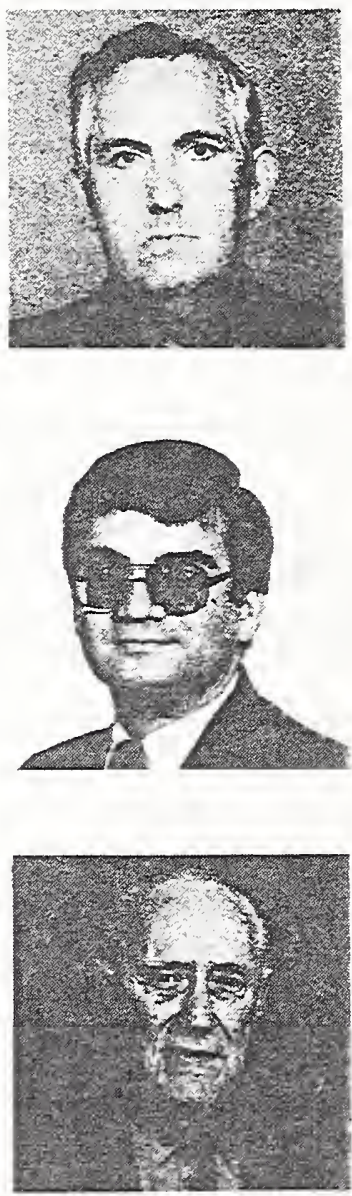

BULLOCK and JUHASZ can be reached at U.S. Army Ballistic Research Laboratory, Aberdeen Proving Ground, MD 21005-5066. NEWHALL can be reached at Harwood Engineering Co., Inc., $455 \mathrm{~S}$. Street, Walpole, MA 02081-2799, 617/668-3600. 
(drop hammer method)

J. Riegebauer

AVL List GmbH Graz, Austria

Abstract:

The dynamic pressure calibrator functioning according to the principle of the drop hammer method, allows the generation of pressure curves, which are similar in amplitude and time $(10-800 \mathrm{MPa}, 2.5 \mathrm{msec})$ to phenomena e.g. occurring in ballistic measurement iechniques. The paper explains the set-up of the calibrator and the possible error influences on the peak pressure are discussed in detail. A calibration method for obtaining traceability is introduced and prospects as to further development work for reaching primary standard are outlined. 


\section{Introduction}

The course of the gas pressure is an important characteristic magnitude for engine indication and internal ballistic measurements techniques. In the past few years piezoelectric pressure transducers have proved to be especially reliable for measuring the course of this characteristic magnitude.

Based on its high resonance frequency $(100-280 \mathrm{kHz})$, the piezoelectric pressure transducer is well suited for measuring these highly dynamic events (ms-range). It is of utmost importance to exactly know the transducer characteristics, in particular, its sensitivity behavior for these measurement requirements. For obtaining the sensitivity characteristics a calibration method is to be applied which largely corresponds to the practical measurement procedure (see [3] fig. 4).

From the above the following requirements can be derived for a dynamic pressure calibrator:

- $\quad$ calibration curve similar in amplitude and time e.g. up to $800 \mathrm{MPa}$, $2-20 \mathrm{msec}$ to the phenomenon to be measured

- $\quad$ short load duration positively effects life time of transducers

- $\quad$ high reproducibility and accuracy

- quick and easy operation

- $\quad$ to make the calibration method traceable

- $\quad$ to become primary standard for dynamic pressure

The present classical calibration method with a static dead weight tester can not fulfill the requirement on the calibration procedure to correspond to the highly dynamic measurement events.

However, the dead weight tester is a primary standard and the required pressure can be generated very precisely, which is an essential advantage.

During the past few years, great efforts have been made by various institutes to also obtain a primary standard for dynamic pressure. So far, this aim has not been reached.

The following different methods are known for generating dynamic pressure:

- ballistic pressure generators (e.g. air gun, closed vessel)

- $\quad$ the principle of the shock wave tube

- $\quad$ negative or positive going pressure step ( $\mu$ sec-range)

- drop hammer methods etc.

From these, AVL chose the drop hammer method for developing the dynamic pressure calibrator. 


\section{Principle of pressure generation and calibrator set-up}

The impact mass $M$ drops from the falling height (see fig. 1) on the piston $m$, which seals the hydraulic pressure chamber. The piston and pressure chamber assembly represent the measuring head $\mathrm{MH}$ equipped with the pressure transducers to be calibrated. At the impact of the impact mass the kinetic energy is converted into compression energy by ingressing of the piston into the pressure chamber. During transition from the compression phase into the expansion phase the maximum pressure $p_{\max }$ is reached.

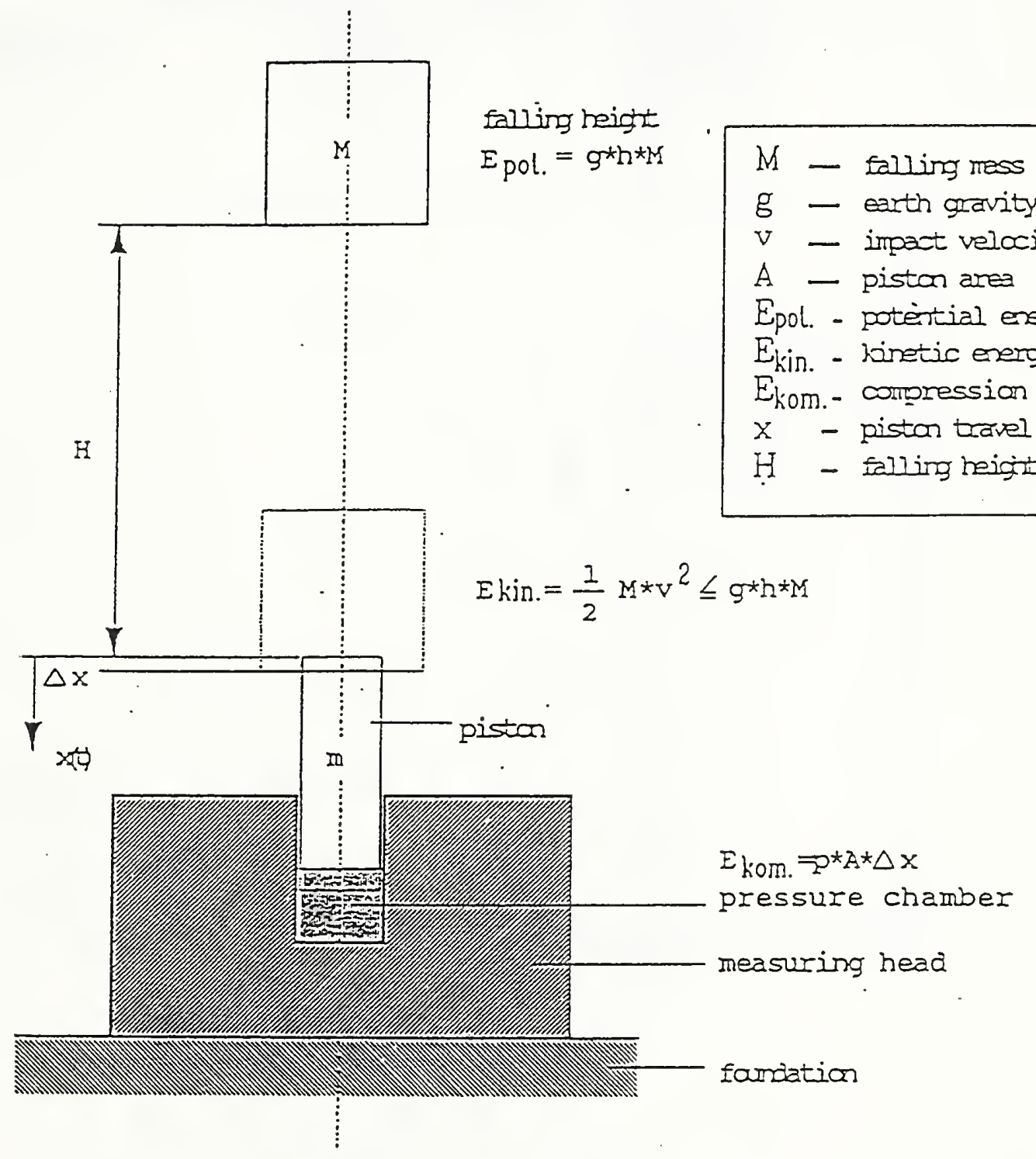

Fig. 1 Principle of pressure generation 


\section{Set-up of the calibrator}

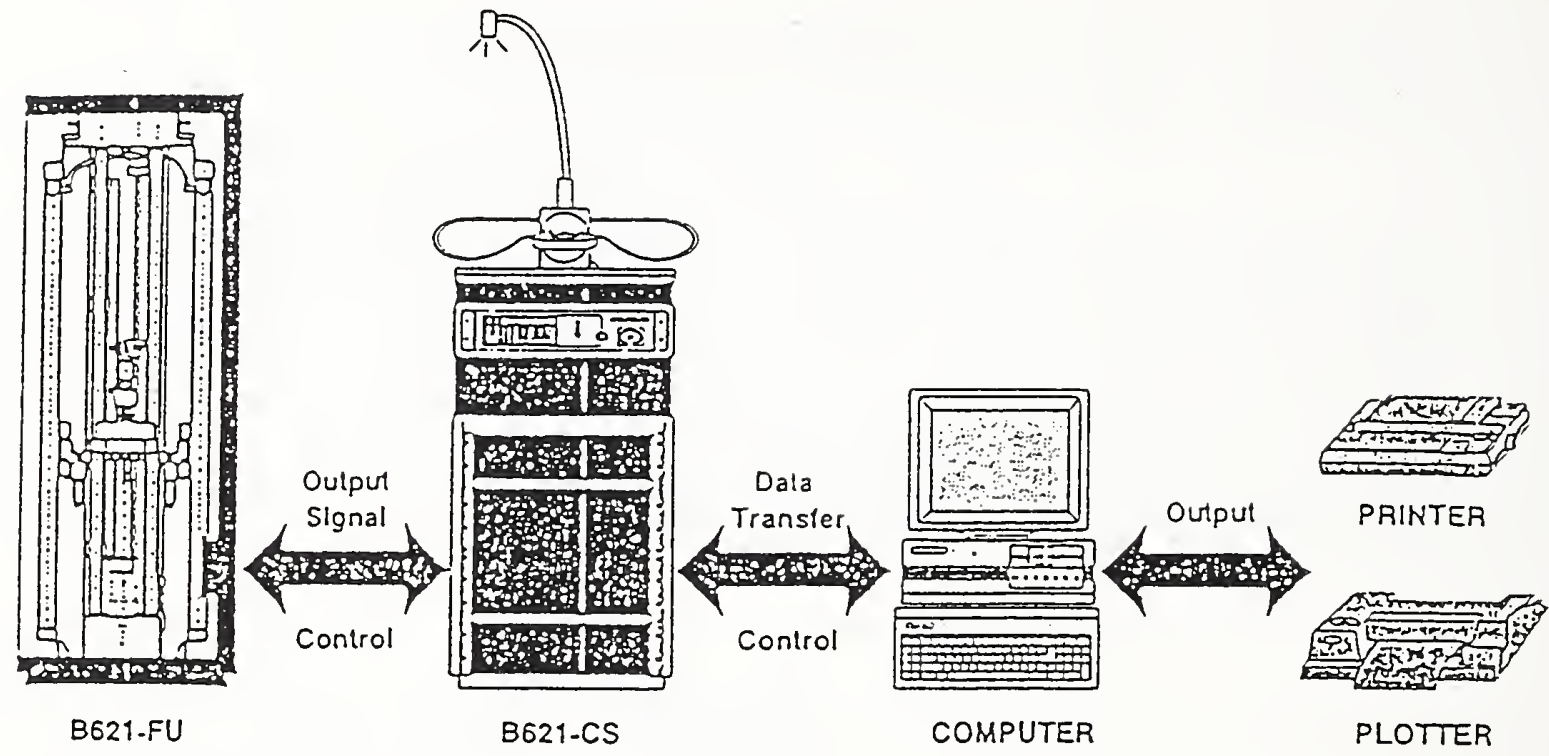

Fig. 2 Complete set-up of the B621 DPC Dynamic Pressure Calibrator

Fig 3 shows all essential B621DPC components and lists the most important magnitudes influencing the pressure generation

Fig. 3 Schematic view of magnitudes influencing the pressure generation

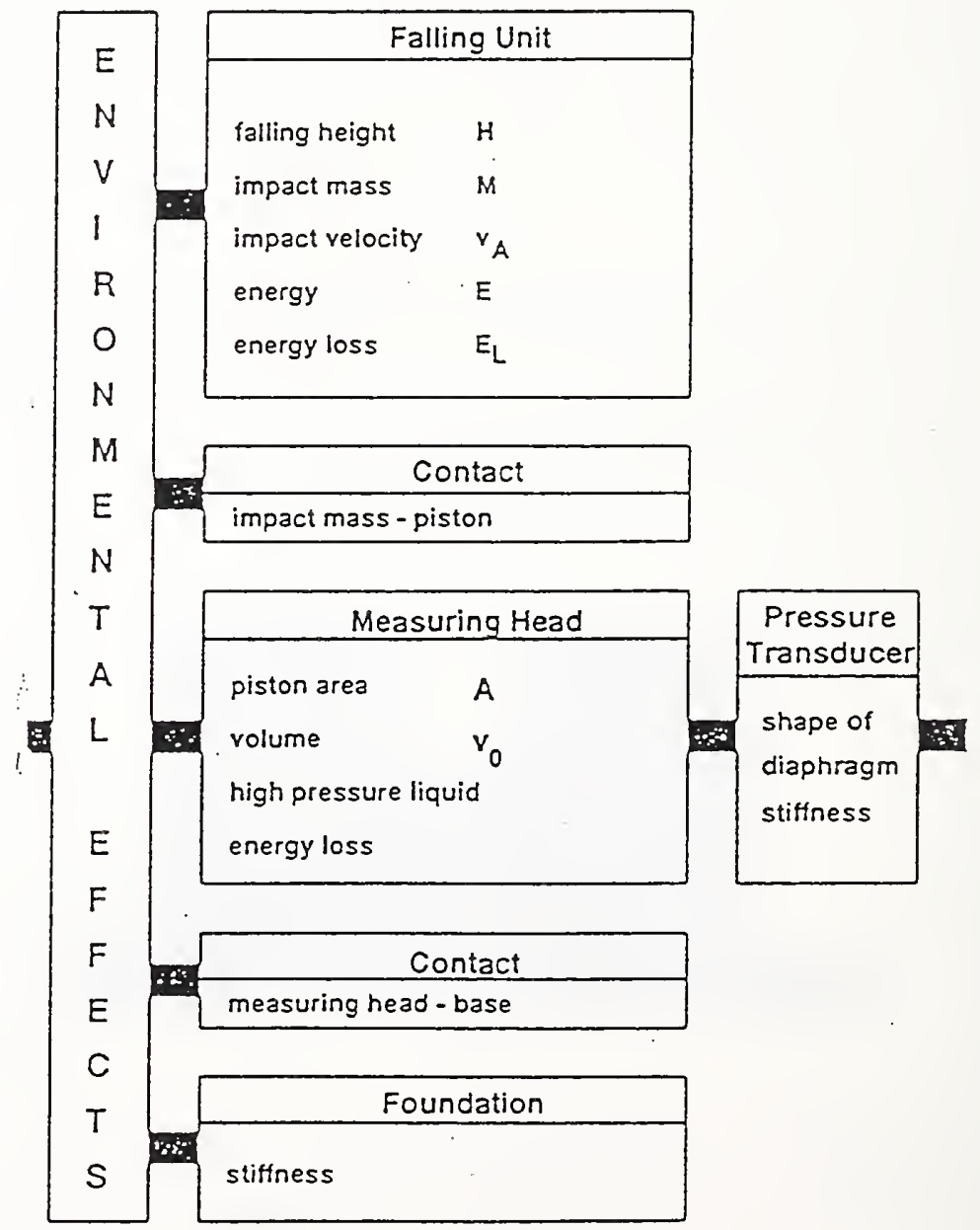


Betow some of these magnitudes will be discussed in detail.

B621 FU Falling Unit

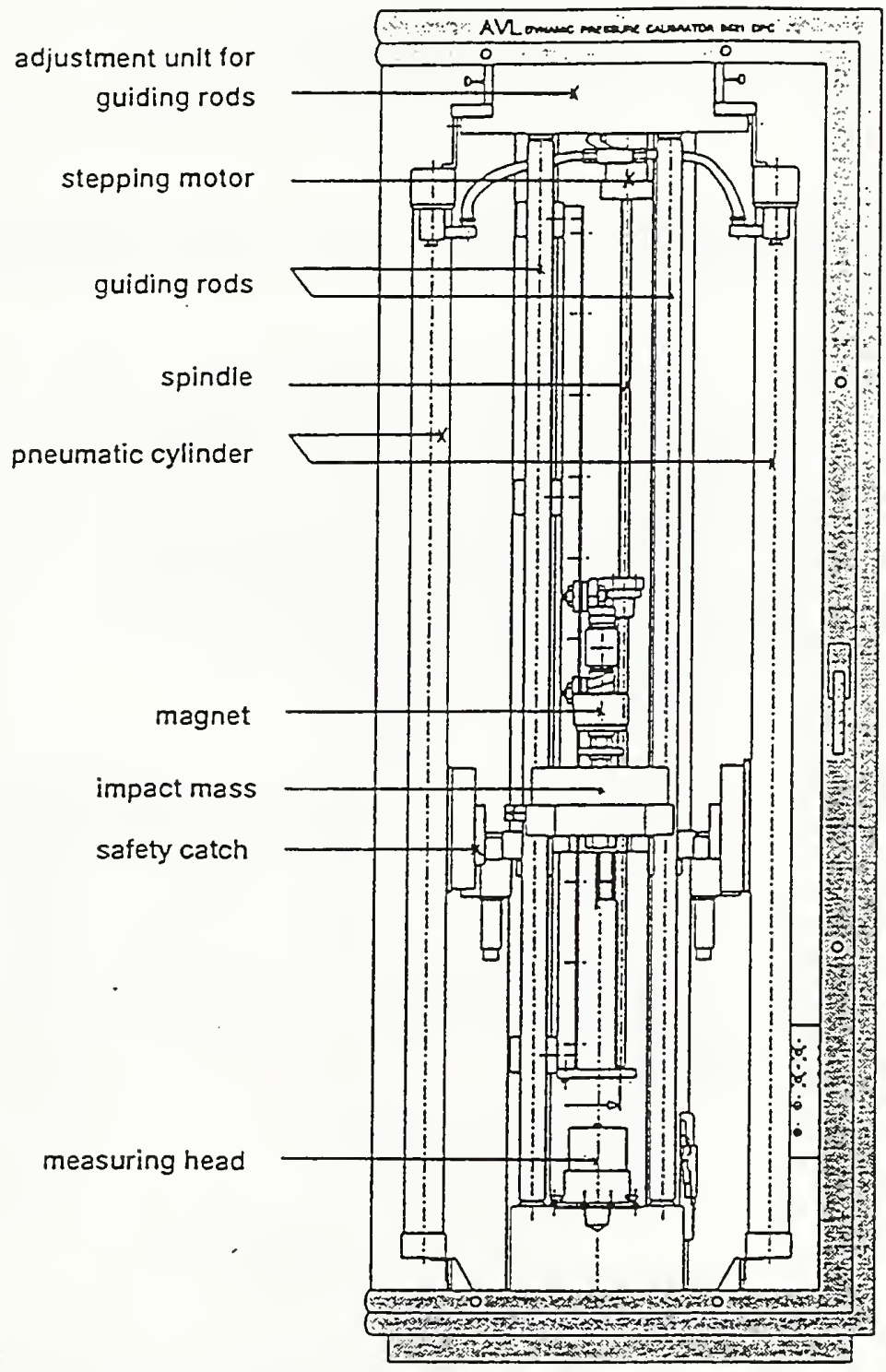

Fig. 4 Schematic arrangement of the B621 FU Falling Unit 


\section{Requirements on the place of installation}

When choosing the place of installation the following conditions have to be met:

- the concrete foundation has to be made on solid ground

- the place of installation must not have cellaring or rooms lying underneath

- $\quad$ the room must be dust-free and free of combustion gases

- room temperature $20^{\circ} \mathrm{C}+1-2^{\circ} \mathrm{C}$

- $\quad$ total space requirement approx. $3.3 \mathrm{~m} \times 3.0 \mathrm{~m}$

- power supply 220 Volt, $50 \mathrm{~Hz}$ or $110 / 120$ Volt, $60 \mathrm{~Hz}$

- compressed air 5 - 12 bar (70 - 170 psi)

Influence of gravitational acceleration at the place of installation on the peak pressure:

The impact mass $M$ drops from the falling height $H$ due to gravitational acceleration in first approximation in free fall and reaches the impact velocity $v_{A}$ directly before hitting the piston. This velocity and the weight of the impact mass depend on the gravitational acceleration. The influence of the gravitational acceleration at the place of installation on the falling height $\mathrm{H}$ for reaching the same impact velocity as at the place of standard $\left(45^{\circ}\right.$ latitude, $0 \mathrm{~m}$ above sea level, comparable with Graz, Austria) is shown in fig. 5.

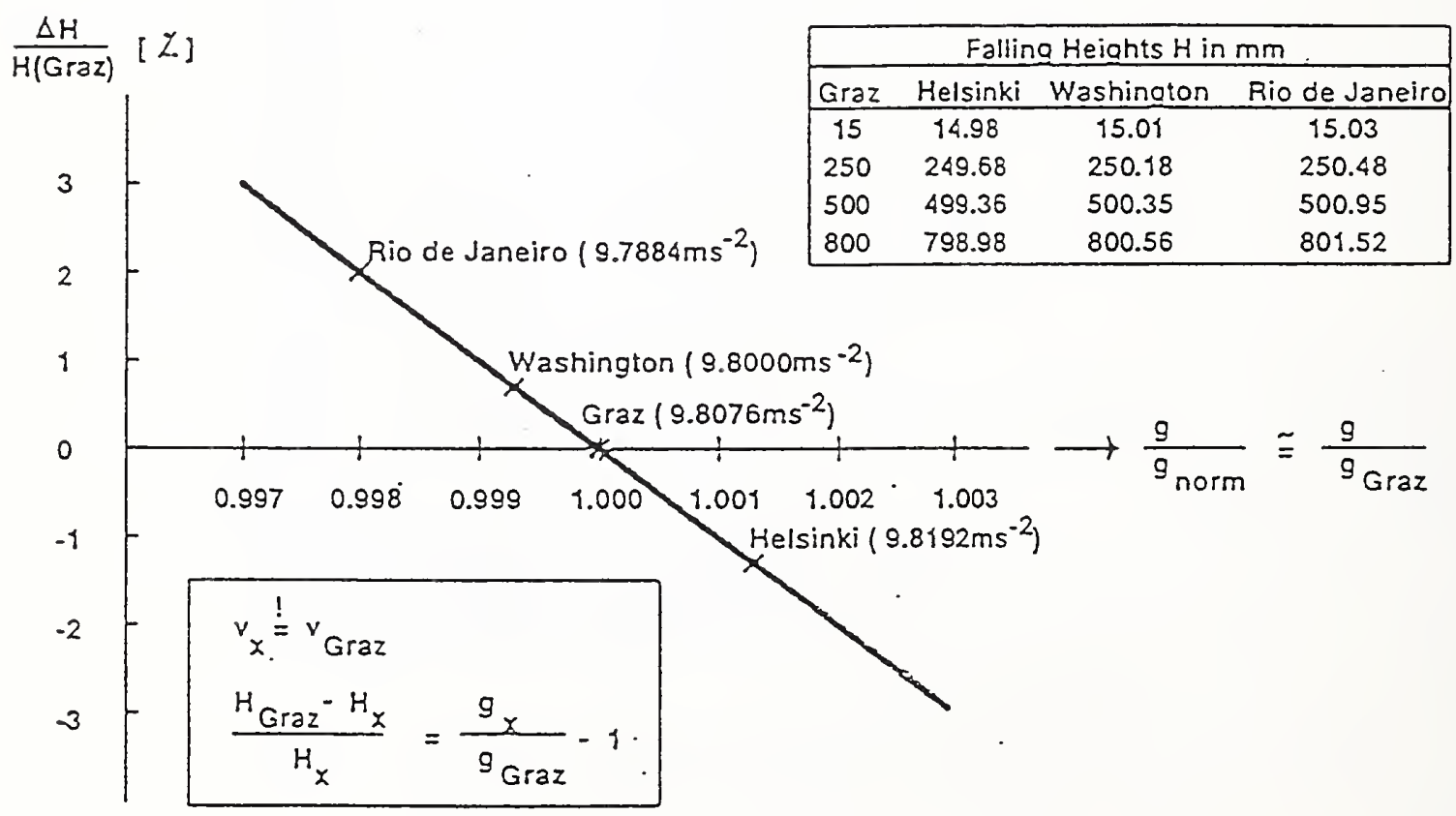

Fig. 5 Influence of gravitational acceleration 


\section{Requirements on the concrete foundation}

The B621 DPC is positioned on a concrete foundation with cast-in steel plate. The foundation acts as counter mass during the impact of the impact mass and makes the impact procedure and thus the pressure generation largely independent from installation conditions such as nature of ground composition, stiffness and stability (see fig. 6).

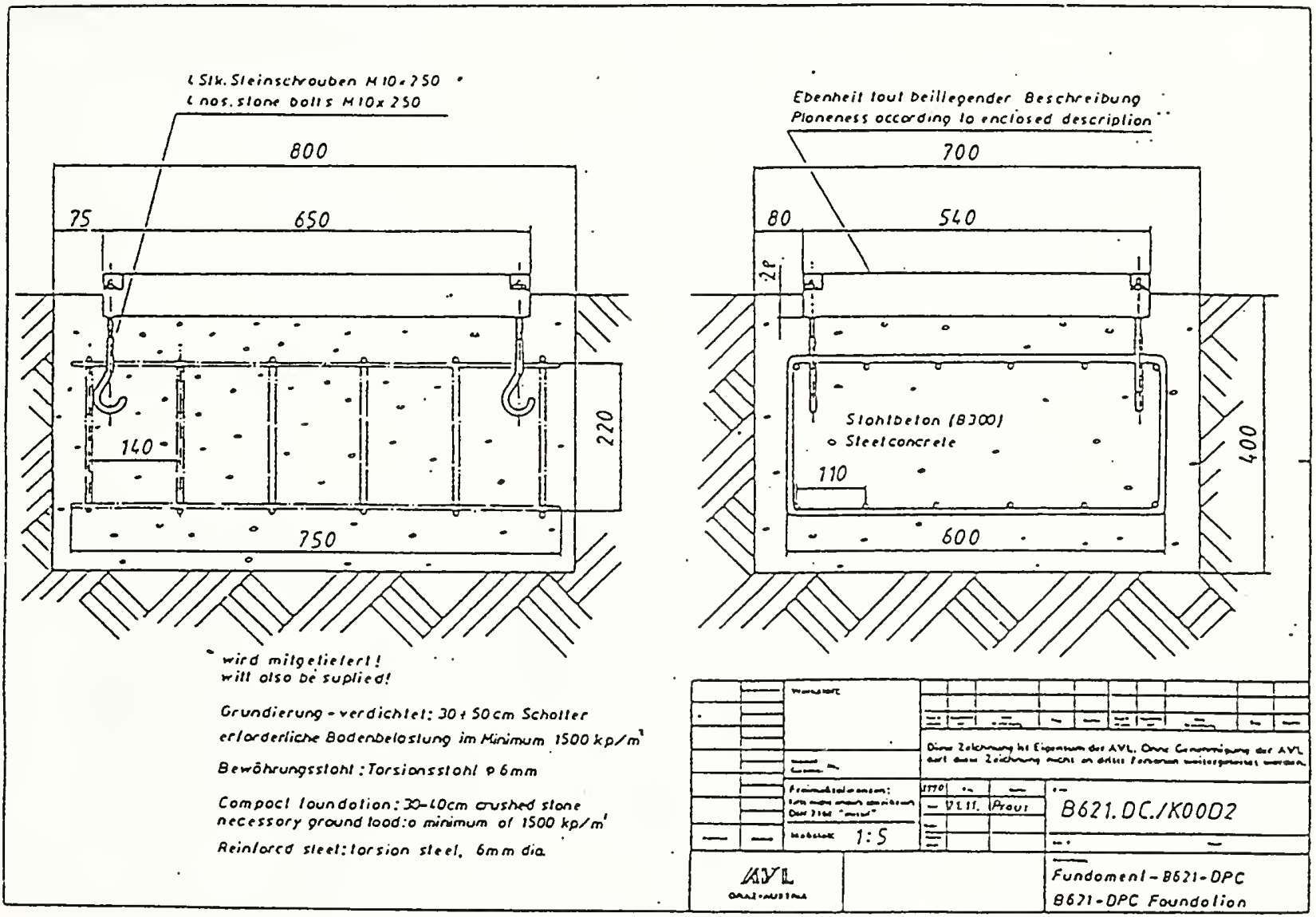

Fig. 6 Constructional drawing of concrete foundation

At the preparation of the foundation the geometric dimensions can only be kept within certain tolerances. These deviations result in a dispersion of the total mass by the nominal value and thus in a dispersion of the peak pressure which can be considered as systematic error from calibrator to calibrator. 
The influence of the total mass of the foundation on the peak pressure and on the ingression depth of the piston is shown on fig. 7.
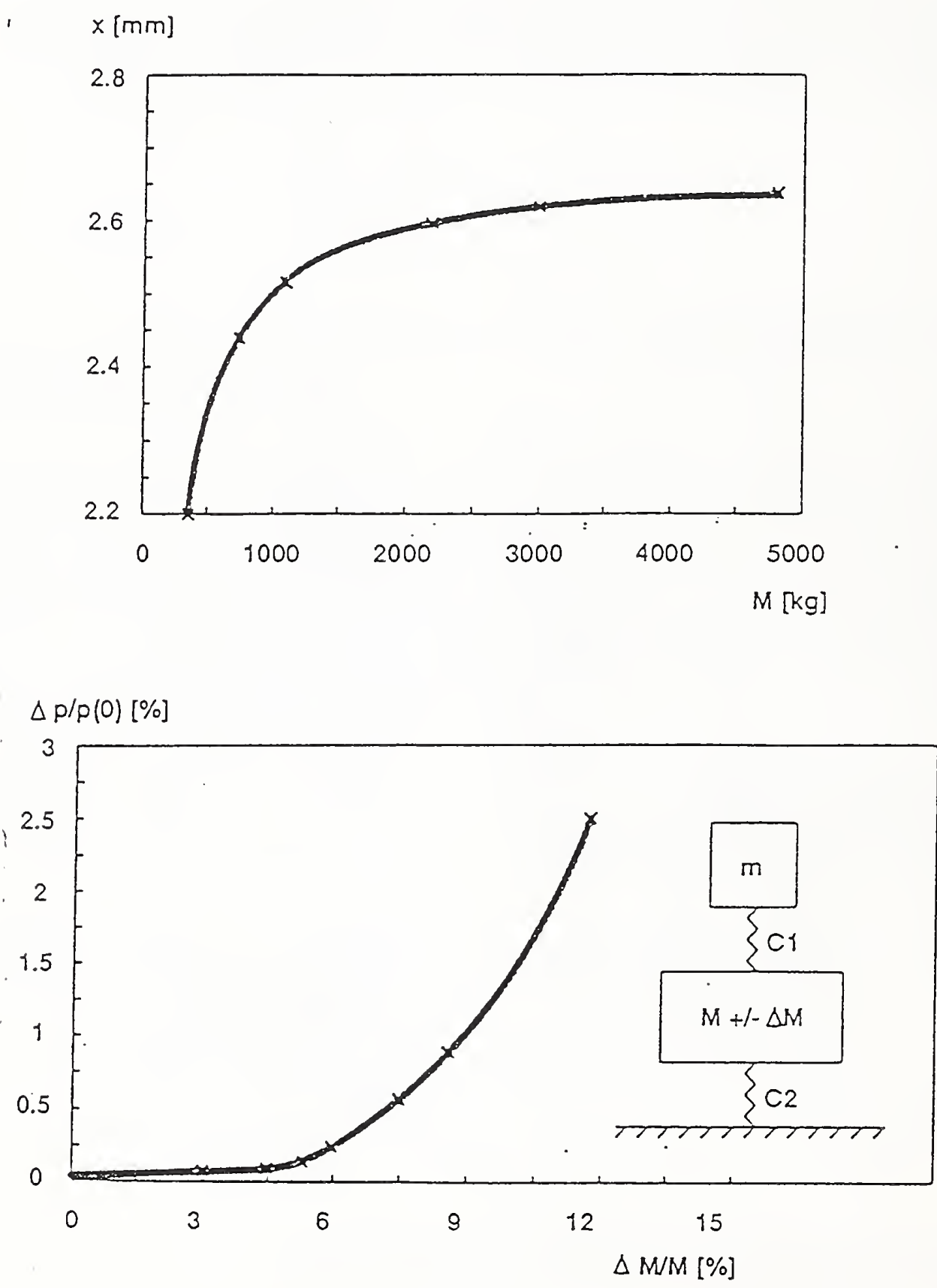

Fig. 7 Influence of foundation mass on the peak pressure

The stiffness of the ground on which the concrete foundation is installed also influences the peak pressure. Therefore make sure that the place of installation has no cellarings or rooms lying underneath (change in peak pressure of approx. $5-10 \%)$. 
Mass of the impact mass

Manufacturing tolerances: $\quad \Delta M / M_{0}<+/-0.140 \%$

Weighing at the Bureau of Standards: $\quad \Delta M / M_{0}<+/-0.001 \%$

Mass of the built-in impact mass

including trigger cable

$$
\begin{aligned}
& \Delta M / M_{0}<+/-0.080 \% \\
& \Delta p / p_{0}<+/-0.040 \%
\end{aligned}
$$

Height adjustment and zeroing

When a pressure value is selected it is converted to the relevant falling height. The impact mass is positioned by means of a stepping motor ( 400 steps per revolution) and the electronic control unit at this falling height. The falling height is calculated beginning from the piston surface i.e. this position has to be determined after switching on the system (zeroing). The positioning repeatability of the impact mass to the falling height selected is $+/-0.02 \mathrm{~mm}$, this value was verified by a laser interferometer. A falling height of $14 \mathrm{~mm}$ corresponds to a relative uncertainty of the height adjustment or the pressure generation of

$$
\begin{aligned}
& \Delta H / H_{0}=+/-0.15 \% \\
& \Delta p / p_{0}=+/-0.08 \%
\end{aligned}
$$

This uncertainty is less than $+/-0.1 \%$ for falling heights over $300 \mathrm{~mm}$.

$$
\left(\Delta p / p_{0}=+/-0.03 \%\right)
$$


Measuring the impact velocity $v_{A}$

A light screen system measures the characteristic time during falling of the impact mass. By means of this time the falling behavior of the impact mass can be checked and extrapolated to the impact velocity. (see fig. 8 )

The uncertainty at measuring the impact velocity is approx. $+1-0.4 \%$

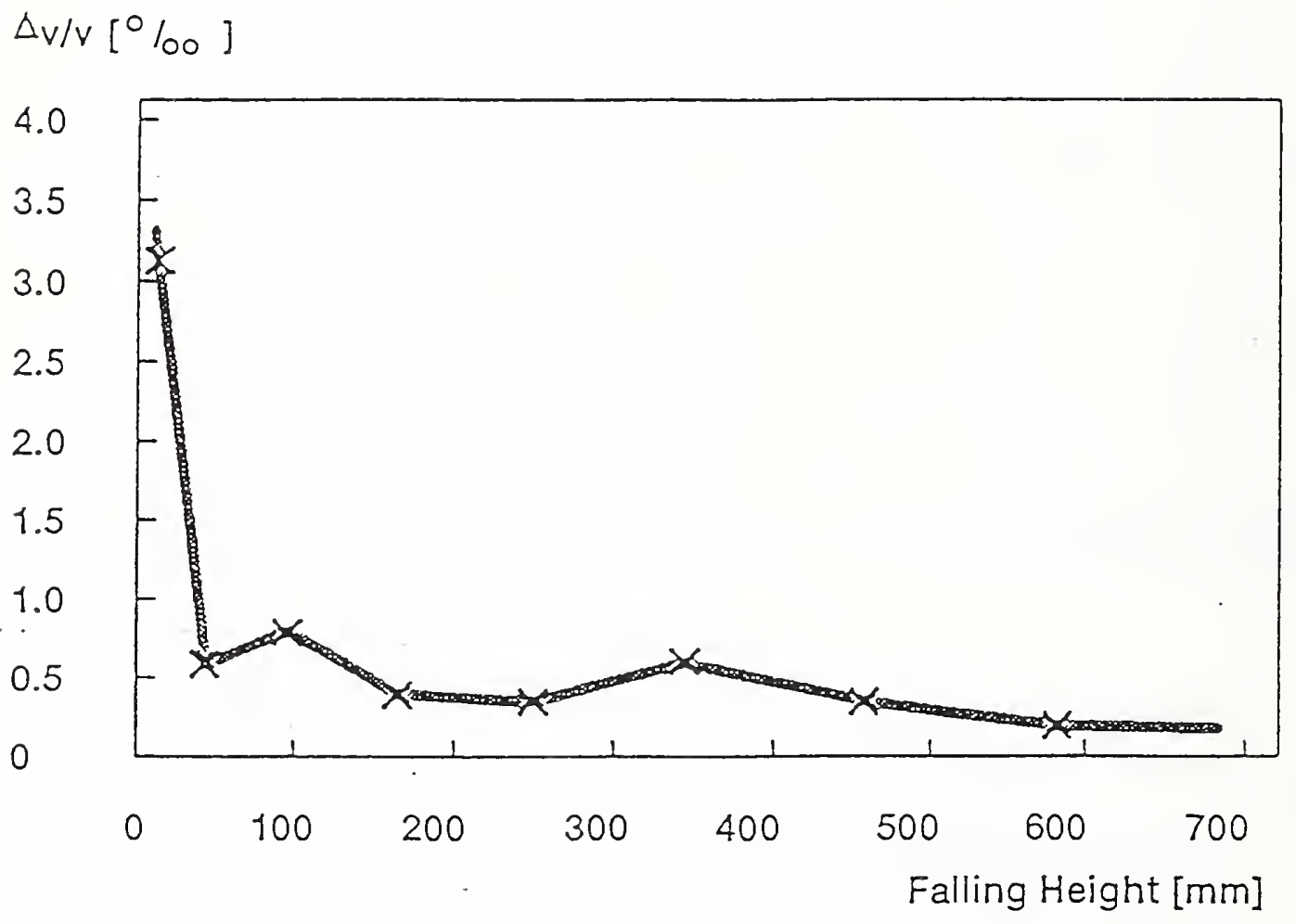

Fig. 8 Measurement uncertainty of impact velocity $v_{A}$

Influence of friction between guiding rods and guide sleeve of the impact mass and of air friction on the impact velocity $v_{A}$

When the measured velocities are compared with the theoretically calculated velocities it deviates by approx. $+/-2-5 \%$.

Out of that the air friction has a quota of approx. $+/-0.03 \%$. As the velocities are measured only the measurement uncertainty is relevant for error considerations (see fig. 8). 


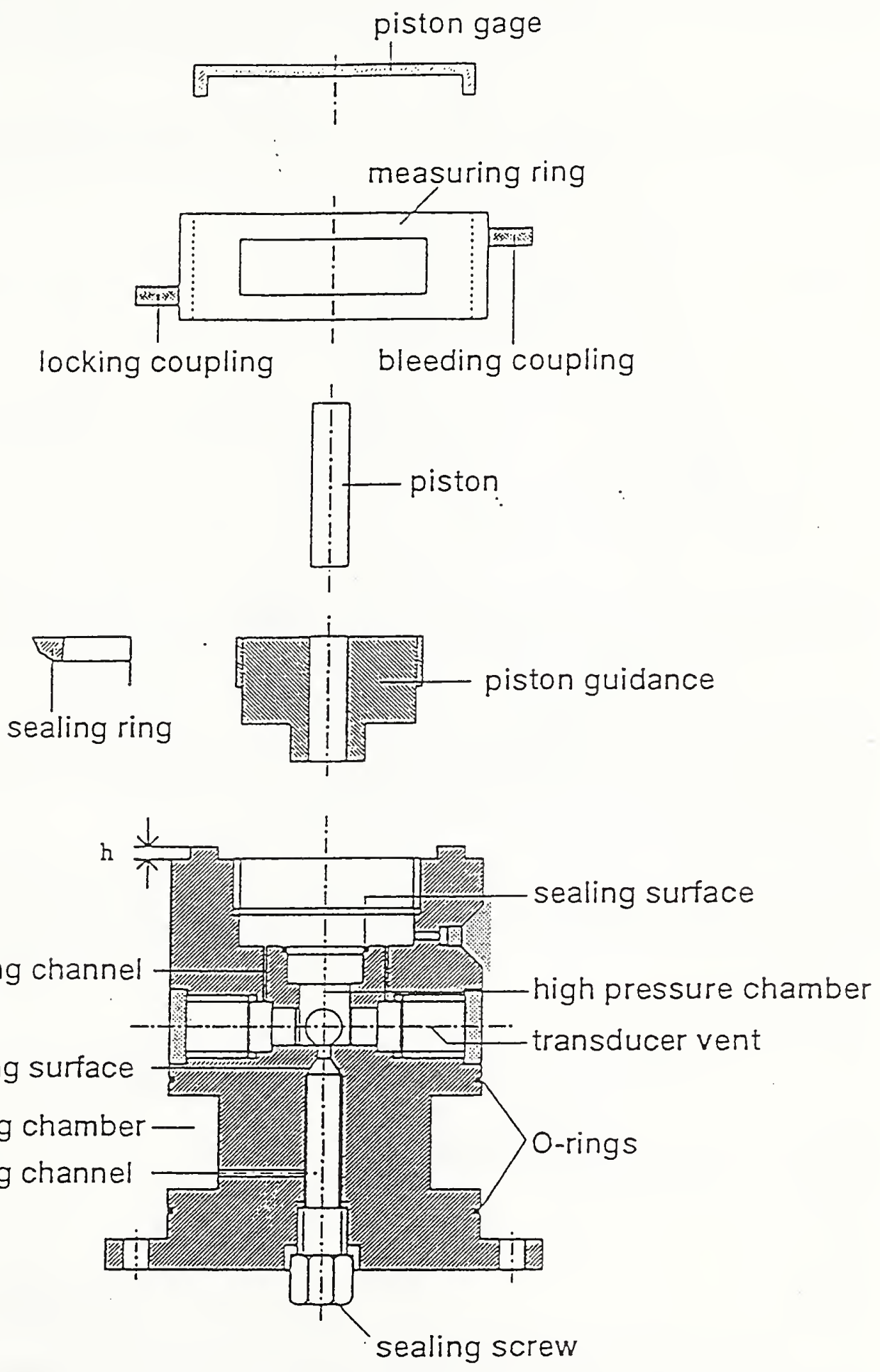

Fig. 9 Set-up of the High Pressure Measuring Head 
Pressure curves generated with B621DPC

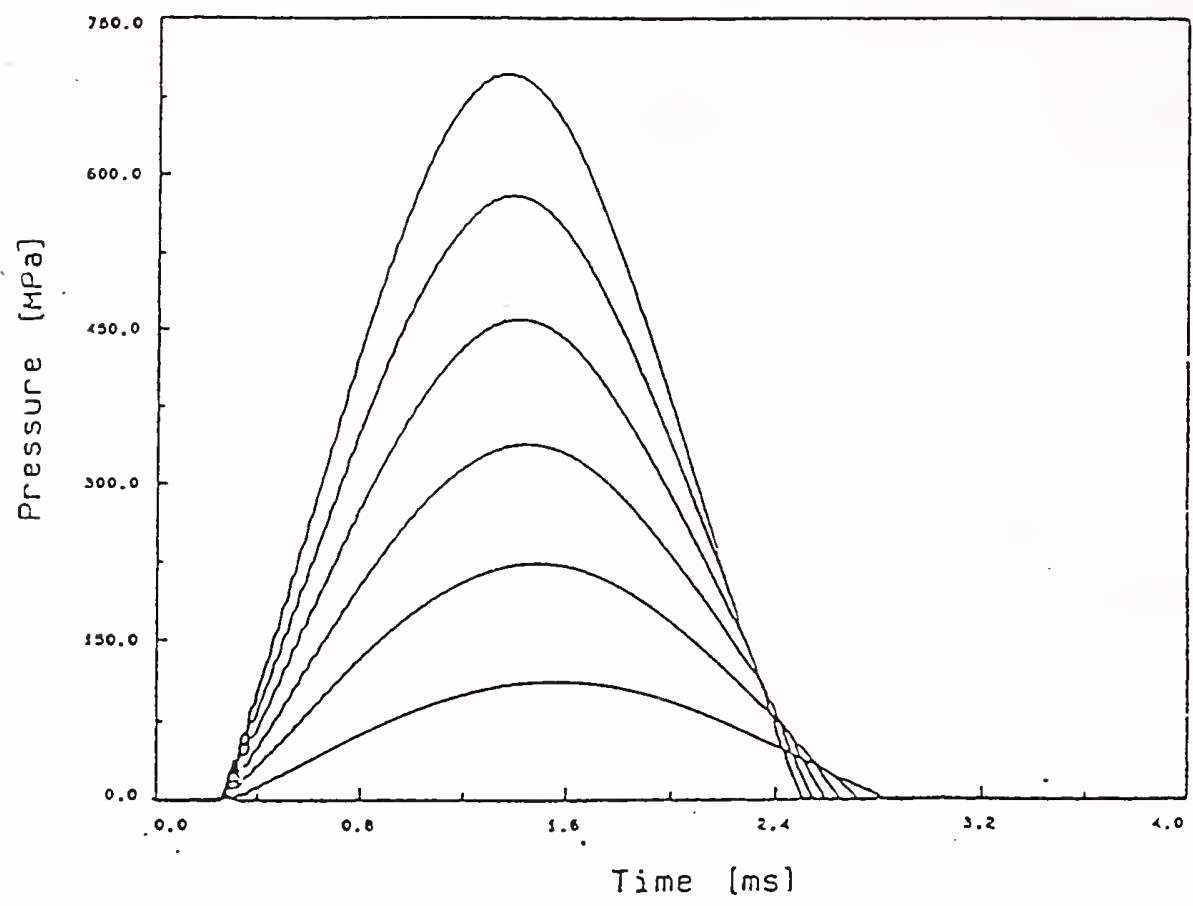

$5006000 \mathrm{~T}$

Fig. 10 Pressure curves generated with B621DPC

Influence of the measuring head volume on the peak pressure

The peak pressure generated depends on the measuring head volume, the dependence is shown on fig. 11 (experimentally determined).

$\mathrm{KH}[\mathrm{mm}]$

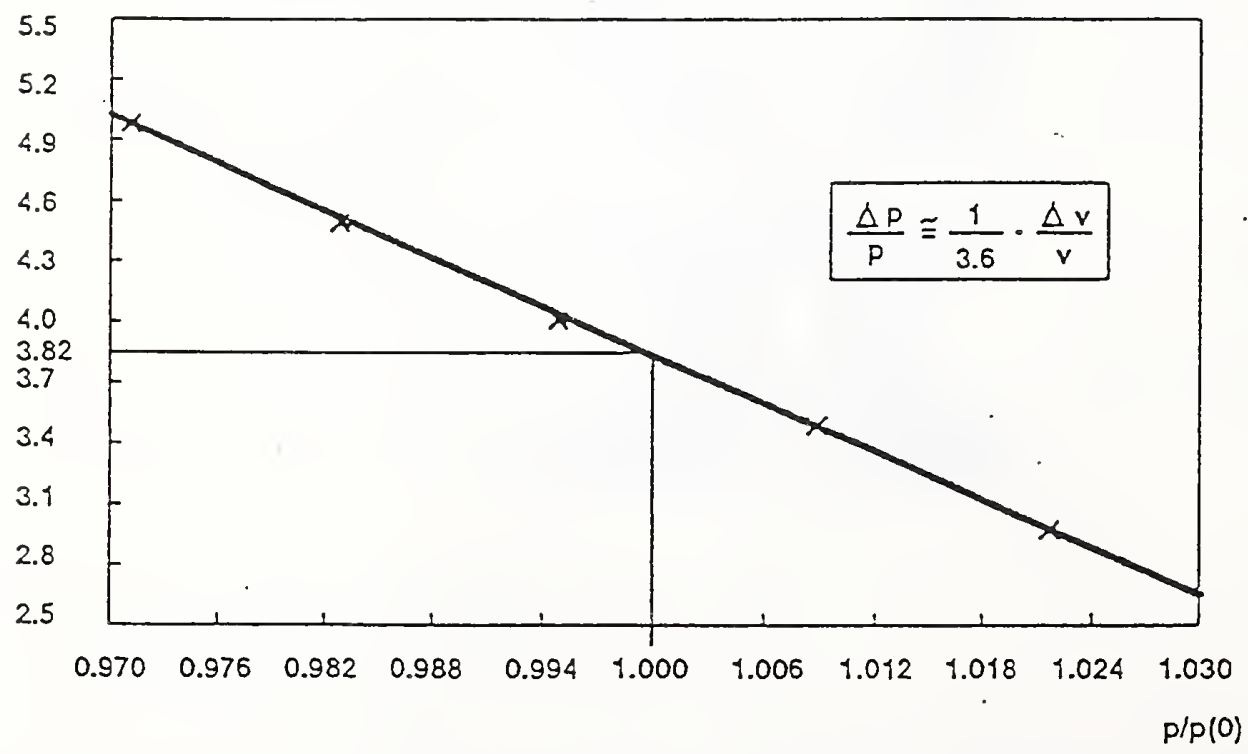

Fig. 11 Dependence of peak pressure on measuring head volume 
When manufacturing the measuring head it is therefore necessary to specify the exact pressure transducer type and to know the dimensions of the front part. Any deviation or change of the original volume, e.g. use of different sealing rings (not in line with the specification), thermal shock diaphragms, mechanical filters etc. leads to a systematic error.

The uncertainty of the measuring head volume due to manufacturing tolerances at known pressure transducer types

$$
\begin{aligned}
& \Delta V=+/-20 \mathrm{~mm}^{3} \\
& \Delta V / V_{0}=+/-2.4 \% \\
& \Delta p / p_{0}=+/-0.7 \%
\end{aligned}
$$

corresponds to an uncertainty piston height:

$$
\Delta K H=+/-0.26 \mathrm{~mm}
$$

When measuring the manufacturing dimensions with a micrometer the uncertainty can be reduced to

$$
\begin{aligned}
& \Delta V=+/-2.6 \mathrm{~mm}^{3} \\
& \Delta V / V_{0}=+/-0.30 \% \\
& \Delta p / p_{0}^{\circ}=+/-0.09 \%
\end{aligned}
$$

When the measuring head had already been calibrated the piston gage always ensures the same piston height. The uncertainty of this method for adjusting the piston height with this piston gage is

$$
\begin{aligned}
& \Delta K H=+/-0.02 \mathrm{~mm} \\
& \Delta V=+/-1.6 \mathrm{~mm}^{3} \\
& \Delta V / V_{0}=+/-0.20 \% \\
& \Delta p / P_{0}=+/-0.05 \%
\end{aligned}
$$

\section{Dead volume of pressure transducers}

Pressure transducers with the same installation dimensions can have a different dead volume (see fig. 12) in the front part, e.g. the transducers of the type AVL 5QP6000T, KISTLER 6211, KISTLER 6203 have a different dead volume of approx. $4 \mathrm{~mm}^{3}$. 
When, for example, these transducers are exchanged during measurement series without considering this fact, it will cause a relative change of the measuring head volume or the peak pressure by approx.

$$
\begin{aligned}
& \Delta V / V_{0}=+/-0.47 \% \\
& \Delta p / p_{0}=+/-0.13 \%
\end{aligned}
$$

transducer

$$
\text { dummy }
$$
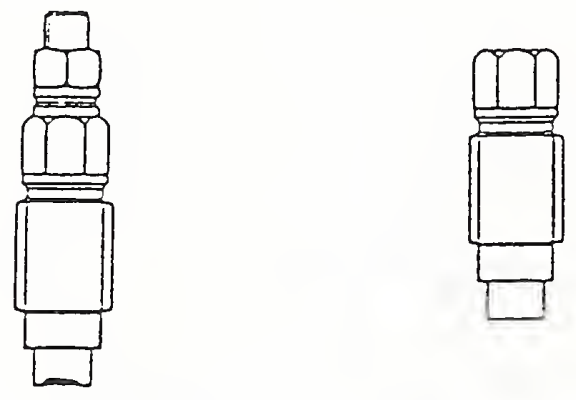

Fig. 12 Dead volume of pressure transducers or dummies

\section{Use of dummies}

Should the dummy not have exactly the shape of the pressure transducer it will result in a relative change of the measuring head volume or the peak pressure by approx.

$$
\begin{aligned}
& \Delta V / V_{0}=+/-1.0-1.4 \% \\
& \Delta p / p_{0}=+/-0.3-0.4 \%
\end{aligned}
$$

A further change in peak pressure derives from the different stress behavior between pressure transducers and dummies (not important at the static calibration) which can be proven experimentally (see fig. 13). Out of four pressure transducers of the same type one was defined as reference pressure transducer and a measurement series was taken. In the 2nd measurement series, the three pressure transducers were replaced by three dummies with their geometric dimensions exactly corresponding to the pressure transducer shapes. The reference pressure transducer was not dismounted from the bore. The difference of the results from the two series was higher than $+1-0.8 \%$. The simplified model of the pressure transducer and the dummy shown on fig. 13 was used for estimating the influence of stress property (Young's modulus E). 
The results can be seen on table 1 .

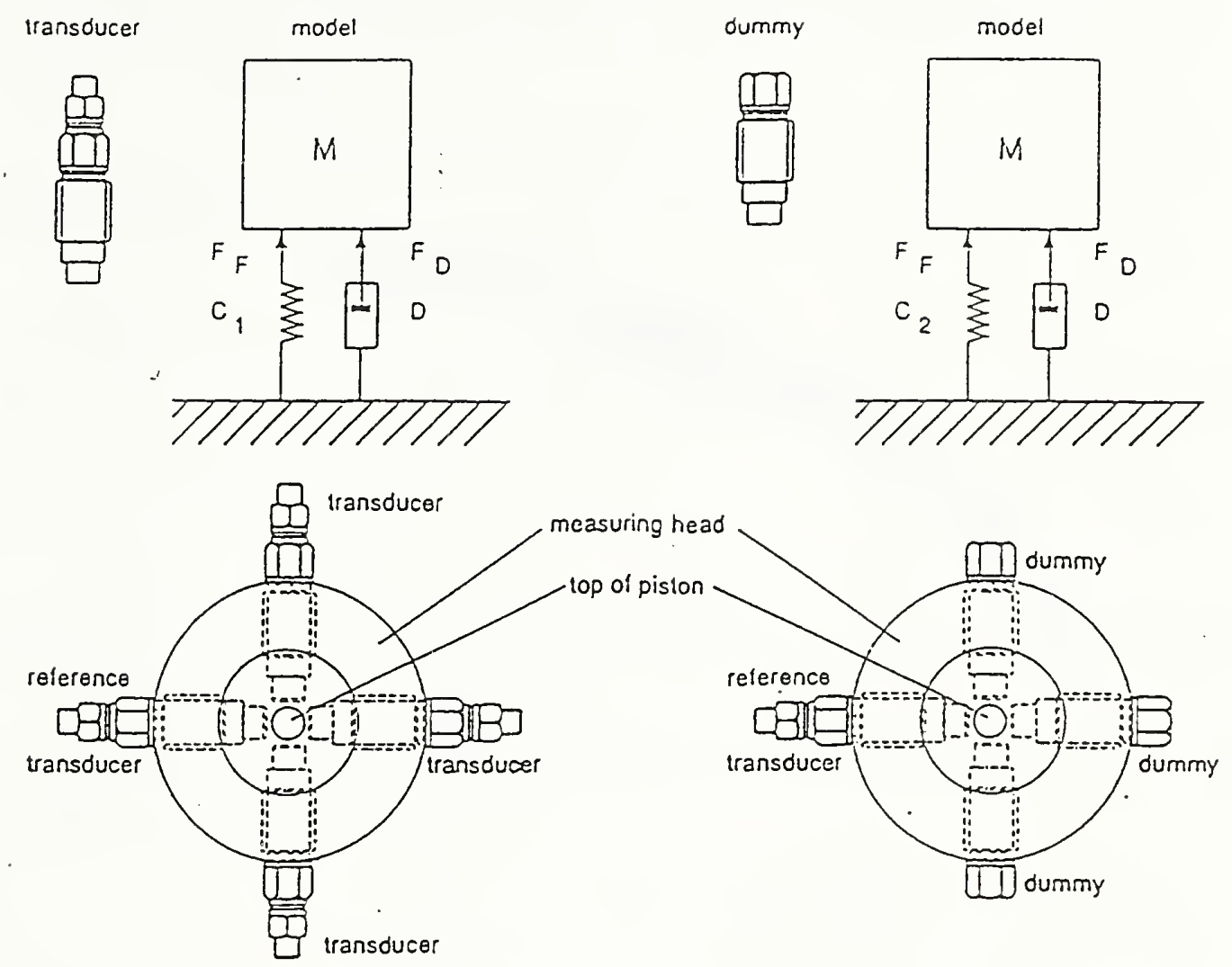

Fig. 13 Simplified model of pressure transducer and dummy and schematic view of experimental set-up

Table 1 E...Young's modulus, $x$ displacement

\begin{tabular}{cll} 
E / GPa & $x / \mu m$ & $\delta x / x_{0} / \%$ \\
\hline 85.000 & 0.992 & 0.0 \\
84.575 & 0.996 & 0.4 \\
84.150 & 1.001 & 0.9 \\
80.750 & 1.037 & 4.4
\end{tabular}

Uncertainty when determining the piston area

$$
\Delta A / A_{0}=2 \cdot \Delta D / D_{0}=2 \cdot 0.005 / 10=+/-0.001 \%
$$

This value is true for the piston not exposed to pressure. 
When the contact surface between measuring head bottom and base bottom of the B621FU is not cleaned, e.g. from drops of liquid, chip grains, etc. it will lead to a peak pressure error. Fig. 14 shows this error (experimentally determined).

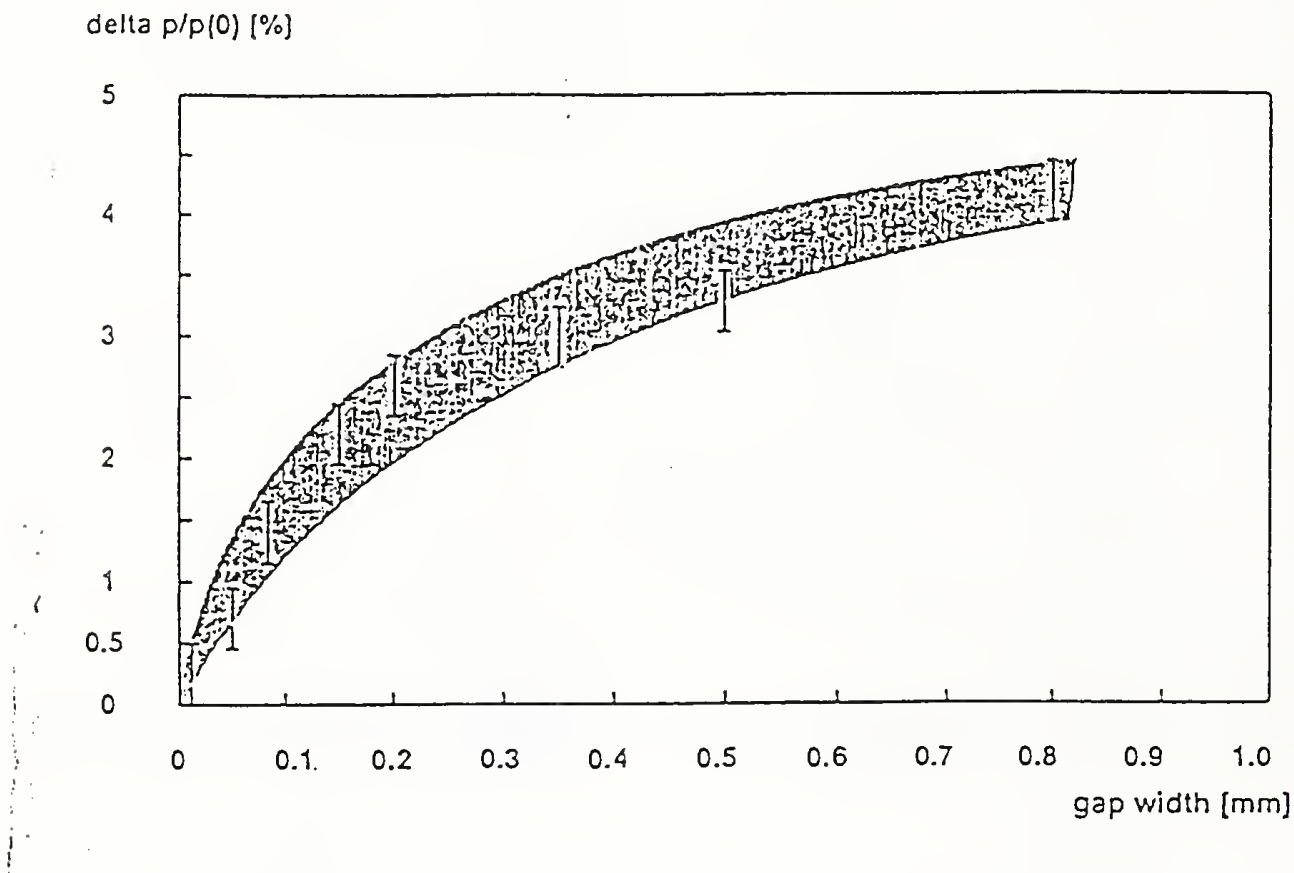

Fig. 14 Inclined contact surface of the measuring head

Air bubbles in the high pressure chamber of the measuring head

Air bubbles influence the shape of the generated pressure curve and its peak pressure. Furthermore, the reproducibility of zeroing is deteriorated which is an additional uncertainty for the height adjustment. Constructional measures on the filling station of the measuring head largely ensure bubble-free filling.

Influence due to leakage of the measuring head on its volume

100 repetitions at $100 \mathrm{MPa}$ and at $600 \mathrm{MPa}$ were made. The piston height was measured with a micrometer before starting the measurement. After completion of the measurement series a difference of $50 \mu \mathrm{m}$ in the piston height was measured, this corresponds to a relative change in volume or pressure of

$$
\begin{aligned}
& \Delta V / V_{0}=+/-0.4 \% \\
& \Delta p / p_{0}=+/-0.1 \%
\end{aligned}
$$


As approx. 15 repetitions are made at a typical calibration cycle, this error influence is very low i.e. less than $+/-0.015 \%$.

Influence of the ambient temperature on the measuring head

Change of piston area

A temperature change of $2^{\circ} \mathrm{C}$ results in a relative change of the piston area of approx. $0.005 \%$.

Change of measuring head volume

A temperature change of $2^{\circ} \mathrm{C}$ results in a relative change of the measuring head volume of approx. $0.007 \%$.

Change of compressibility

A temperature change of $2^{\circ} \mathrm{C}$ results in a relative change of the compressibility of the high pressure liquid of approx. $0.6 \%$.

The total influence of the temperature change on the peak pressure can be seen on fig. 15 (experimentally determined)

$$
\Delta p / p_{0}=+/-0.15 \% \text { per }-/+1^{\circ} \mathrm{C}
$$

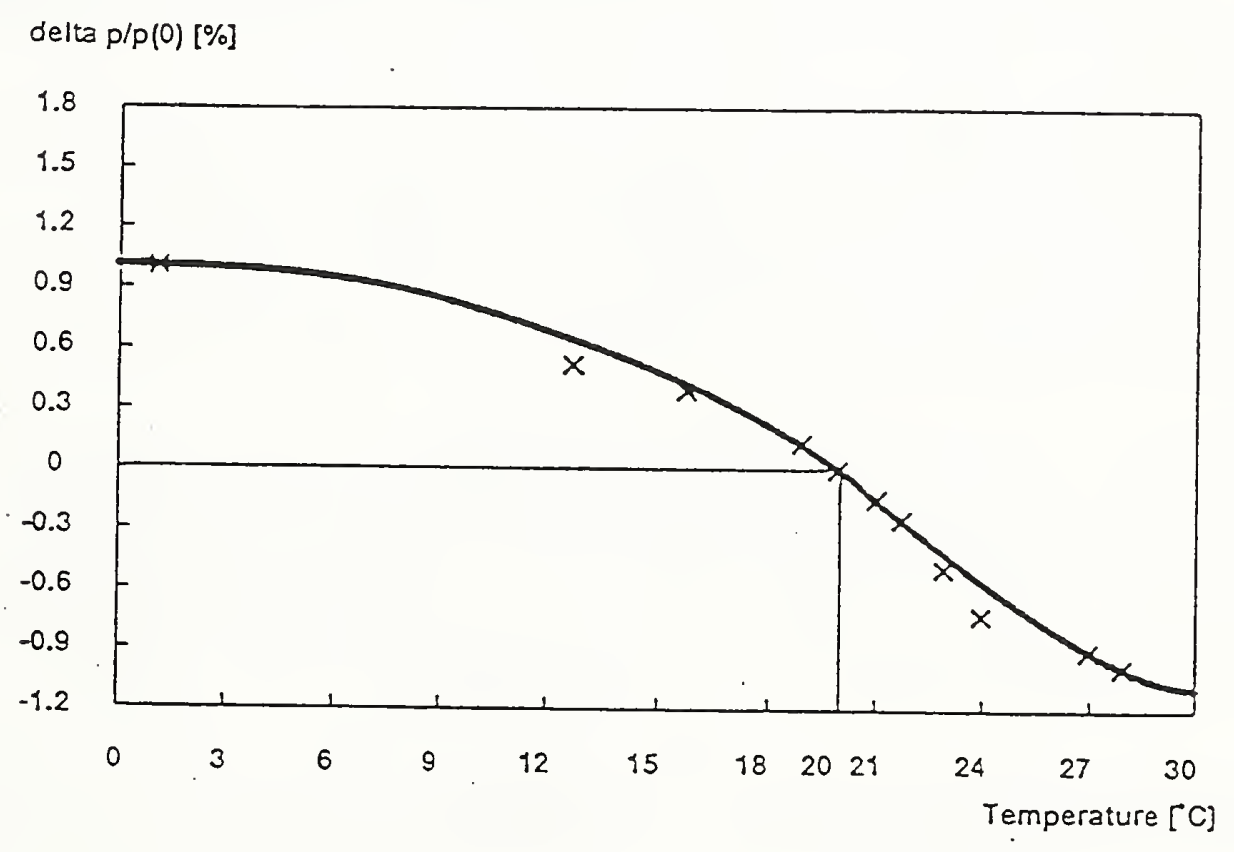

Fig. 15 Temperature influence on peak pressure 
Electronic measurement chain for the dynamic calibration (especially for piezoelectric pressure transducers):

Piezoelectric pressure transducers deliver electric charges at compressive load (see fig. 16). Charge amplifiers convert this charge into voltage, which is proportional to the charge. As the dynamic calibration takes place within approx. $2-5 \mathrm{msec}$, the voltage signal has to be digitized by a high-speed ADD converter (12 bit resolution, $1 \mu \mathrm{sec}$ sampling rate) for this fast event and the measured values have to be stored in the memory e.g. $16 \mathrm{kByte}$ (transient recorders, digital storage oscilloscopes).

Measurement Chain for Dynamic Calibration

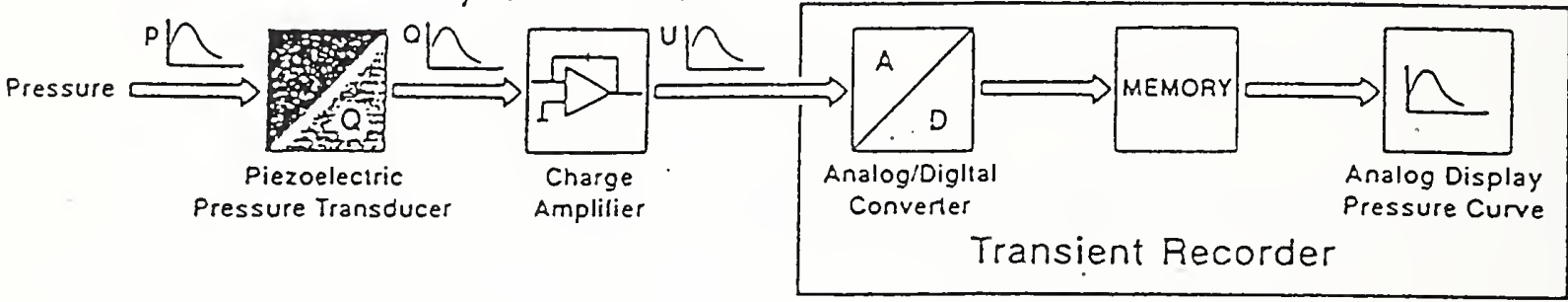

Fig. 16 Principle of the electronic measurement chain for the dynamic pressure measurement

A highly accurate voltage source $U$ and a highly accurate capacitor $C$ generate the reference charge $Q=C * U$ for calibrating the measurement chain.

Accuracy of the charge amplifier and AD-Converter (range $600-20000 \mathrm{pC}$ )

Uncertainty $+/-0.35 \%$ $+/-0.03 \%$

Accuracy of the charge calibrator

Voltage source (B692.A01): $+1-0.050 \%$

or

Voltage source (B201):

$+1-0.100 \%$ calibration capacitor (1000 pC) $+-0.100 \%$ 
Uncertainty of the electronic measurement chain

$$
+/-0.23 \%
$$

Total repeatability within a measurement series

Four pressure transducers were installed into the measuring head and 50 repetitions were made for each pressure step. The pressure transducers were not dismounted during the complete measurement procedure.

Table 2: Repeatability within a series

\begin{tabular}{lllll}
$\begin{array}{l}\text { Step } \\
{[\mathrm{MPa}]}\end{array}$ & $\begin{array}{l}\mathrm{P}_{\text {mean }} \\
{[\mathrm{MPa}]}\end{array}$ & $\begin{array}{l}\sigma \\
{[\mathrm{MPa}]}\end{array}$ & $\begin{array}{l}\text { Range } \\
{[\mathrm{MPa}]}\end{array}$ & $\begin{array}{l}\text { Range } / \mathrm{P}_{\text {mean }} \\
{[\%]}\end{array}$ \\
\hline 100 & 101.2 & 0.04 & 0.2 & 0.19 \\
300 & 301.5 & 0.07 & 0.3 & 0.10 \\
500 & 501.4 & 0.20 & 0.7 & 0.15 \\
600 & 604.5 & 0.70 & 2.7 & 0.43 \\
$800^{\circ}$ & 792.9 & 0.90 & 2.6 & 0.33
\end{tabular}

Total reproducibility with mounting/dismounting of pressure transducers (30 repetitions per pressure step, 9 combinations with three different types of pressure transducers)

A reference transducer (AVL 5QP6000T) was mounted in one bore of the measuring head. It was not dismounted during the measurement. Similar types of pressure transducers were randomly mounted or dismounted in the remaining three bores (see table 3). 
Tabte 3: Reproducibility within a series by exchanging transducers (reference transducer)

\begin{tabular}{lllll}
$\begin{array}{l}\text { Step } \\
{[\mathrm{MPa}]}\end{array}$ & $\begin{array}{l}\mathrm{P}_{\text {mean }} \\
{[\mathrm{MPa}]}\end{array}$ & $\begin{array}{l}\sigma \\
{[\mathrm{MPa}]}\end{array}$ & $\begin{array}{l}\text { Range } \\
{[\mathrm{MPa}]}\end{array}$ & $\begin{array}{l}\text { Range/P } \\
{[\%]}\end{array}$ \\
\hline 200 & 201.2 & 0.7 & 2.8 & 1.3 \\
400 & 398.5 & $0: 6$ & 2.3 & 0.6 \\
600 & 598.9 & 1.1 & 3.5 & 0.6
\end{tabular}

\section{Methods for calibrating the calibrator}

\section{Mathematical model [2]}

A simplified model (spring-dashpot model) allows to derive the relation between the falling height and the maximum pressure (see figure 17). The mathematical description of this model results in a nonlinear differential equation of second order (nonlinear spring and damping characteristics).

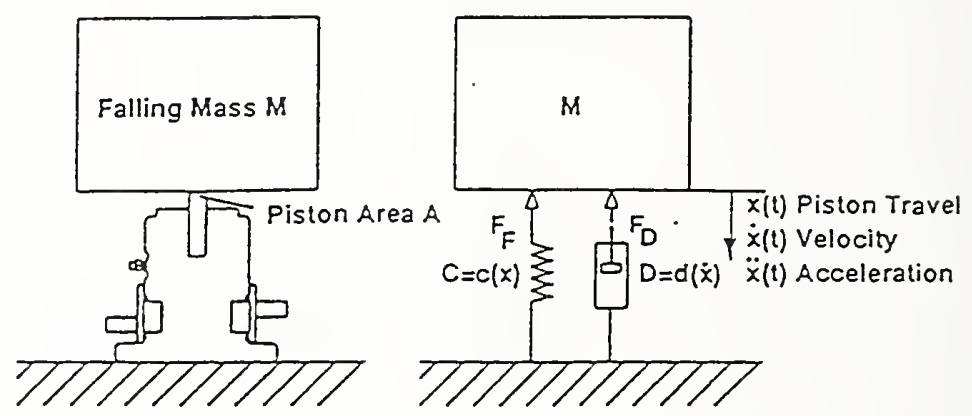

$M \cdot \ddot{x}(t)+D[\dot{x}(t)] \cdot \dot{x}(t)+C[x(t)] \cdot x(t)=0$ $P(t)=\frac{x(t)-C[x(t))]}{A}$

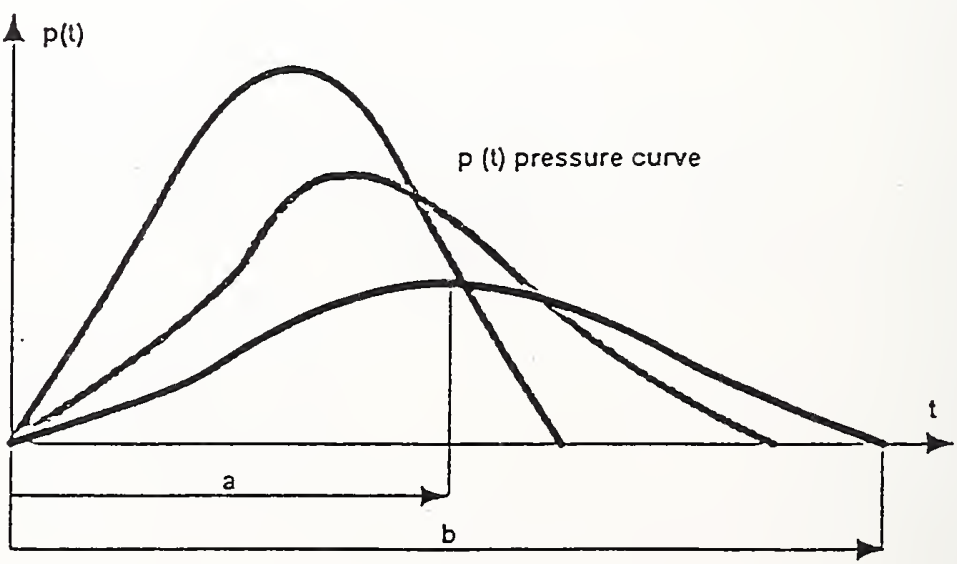

Fig. 17 Mathematical model 
The experimentally derived spring and damping characteristics allow a numerical solution of the equation (see fig. 18).

Characteristic of Spring Stiffness

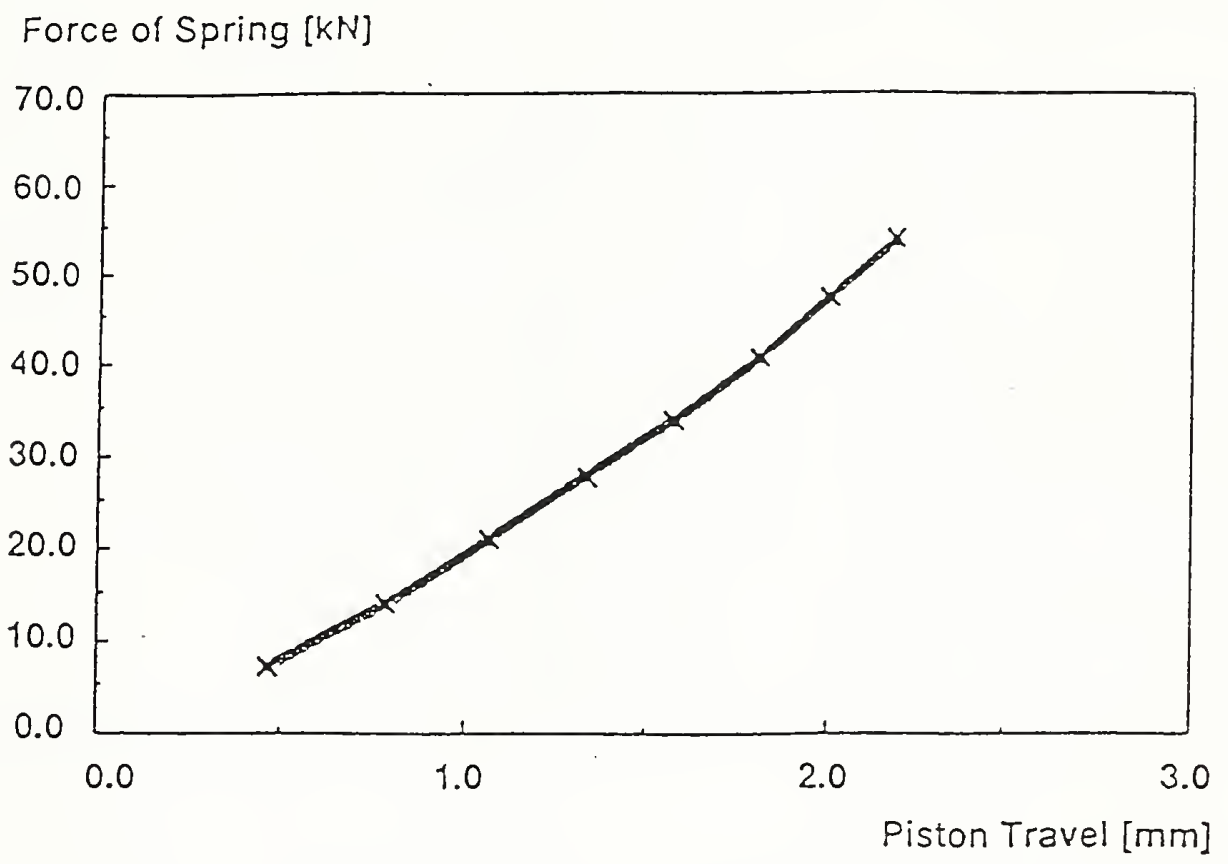

Characteristic of Damping

Force of Damping [kN]

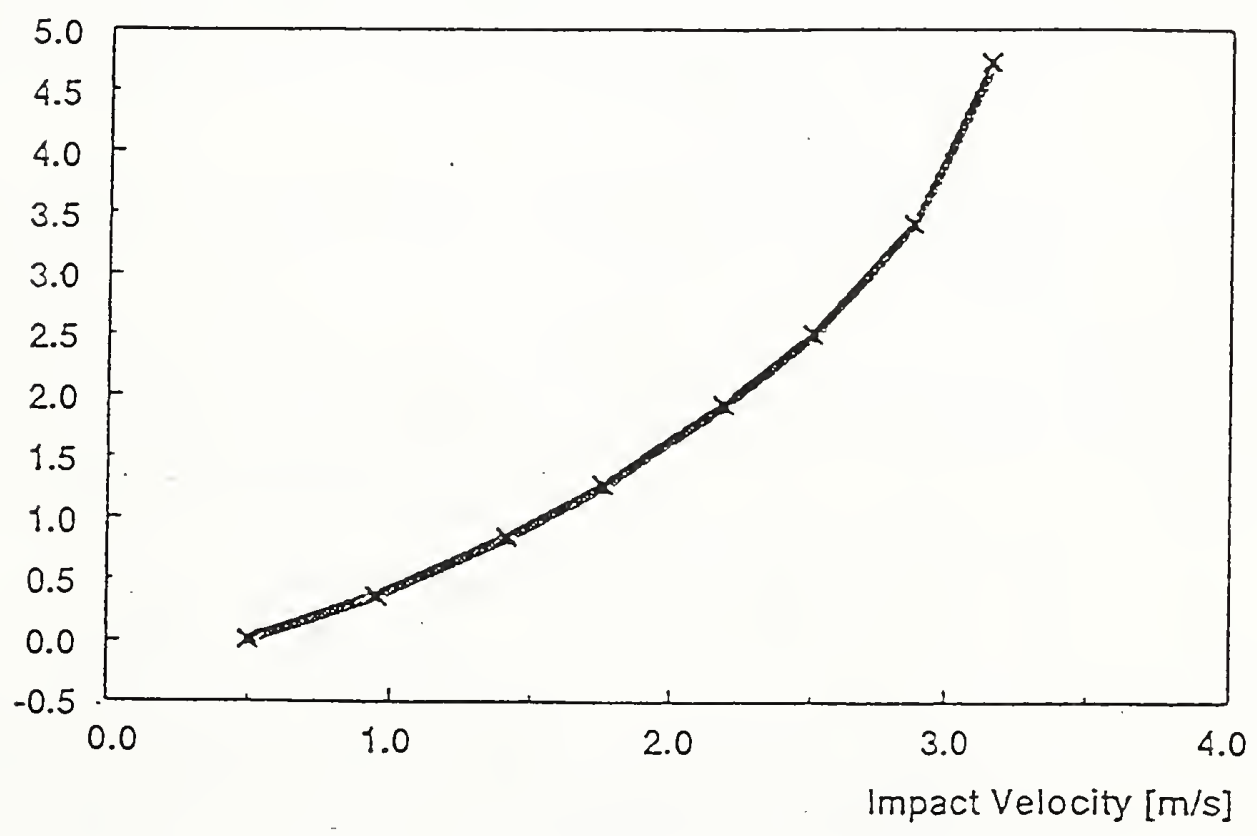

Fig. 18 Spring and stiffness characteristics 
The-result is the mathematical dependency of falling height to generated pressure, see table 4 and fig. 19/20.

Table 4: Results

\begin{tabular}{|c|c|c|c|c|c|c|c|c|}
\hline $\begin{array}{l}\text { Step } \\
\text { [bar] }\end{array}$ & $\begin{array}{l}\mathrm{H} \\
{[\mathrm{mm}]}\end{array}$ & $\begin{array}{l}P_{\text {meas }} \\
\text { [bar] }\end{array}$ & $\begin{array}{l}P_{\text {eval }} \\
\text { [bar] }\end{array}$ & $\begin{array}{l}\varepsilon: \\
{[\%]}\end{array}$ & $\begin{array}{l}T_{\text {gale }} \\
\text { [ms] }\end{array}$ & $\begin{array}{l}V_{A} \\
{[\mathrm{~m} / \mathrm{s}]}\end{array}$ & $\begin{array}{l}T_{A} \\
{[m s]}\end{array}$ & $\begin{array}{l}T_{B} \\
{[\mathrm{~ms}]}\end{array}$ \\
\hline 000 & 13.58 & 949 & 991 & 0.9 & 13.87 & 0.505 & 1.303 & 2.635 \\
\hline 000 & 48.90 & 1912 & 2004 & -0.2 & 6.47 & 0.973 & 1.250 & 2.535 \\
\hline 000 & 102.16 & 2903 & 2989 & 0.4 & 4.41 & 1.401 & 1.214 & 2.470 \\
\hline 000 & 171.28 & 3911 & 4001 & 0.0 & 3.39 & 1.810 & 1.180 & 2.408 \\
\hline 000 & 254.96 & 4927 & 4998 & 0.1 & 2.78 & 2.200 & 1.152 & 2.355 \\
\hline 00 & 352.61 & 5956 & 6000 & 0.0 & 2.37 & 2.577 & 1.128 & 2.306 \\
\hline 00 & 464.42 & 6994 & 7004 & -0.1 & 2.08 & 2.942 & 1.106 & 2.261 \\
\hline 00 & 591.30 & 8049 & 8031 & -0.4 & 1.85 & 3.302 & 1.085 & .221 \\
\hline
\end{tabular}

$\mathrm{P}[\mathrm{MPa}]$

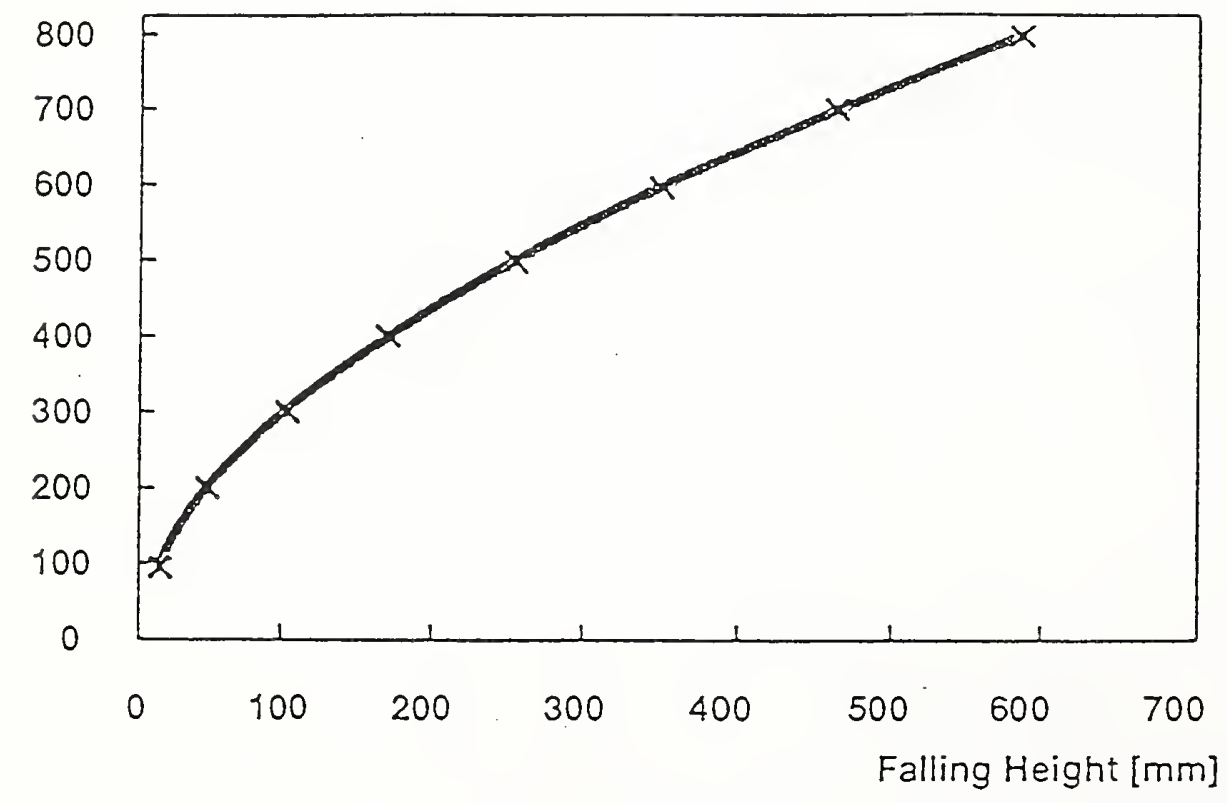

Fig. 19 Dependence pressure - falling height 


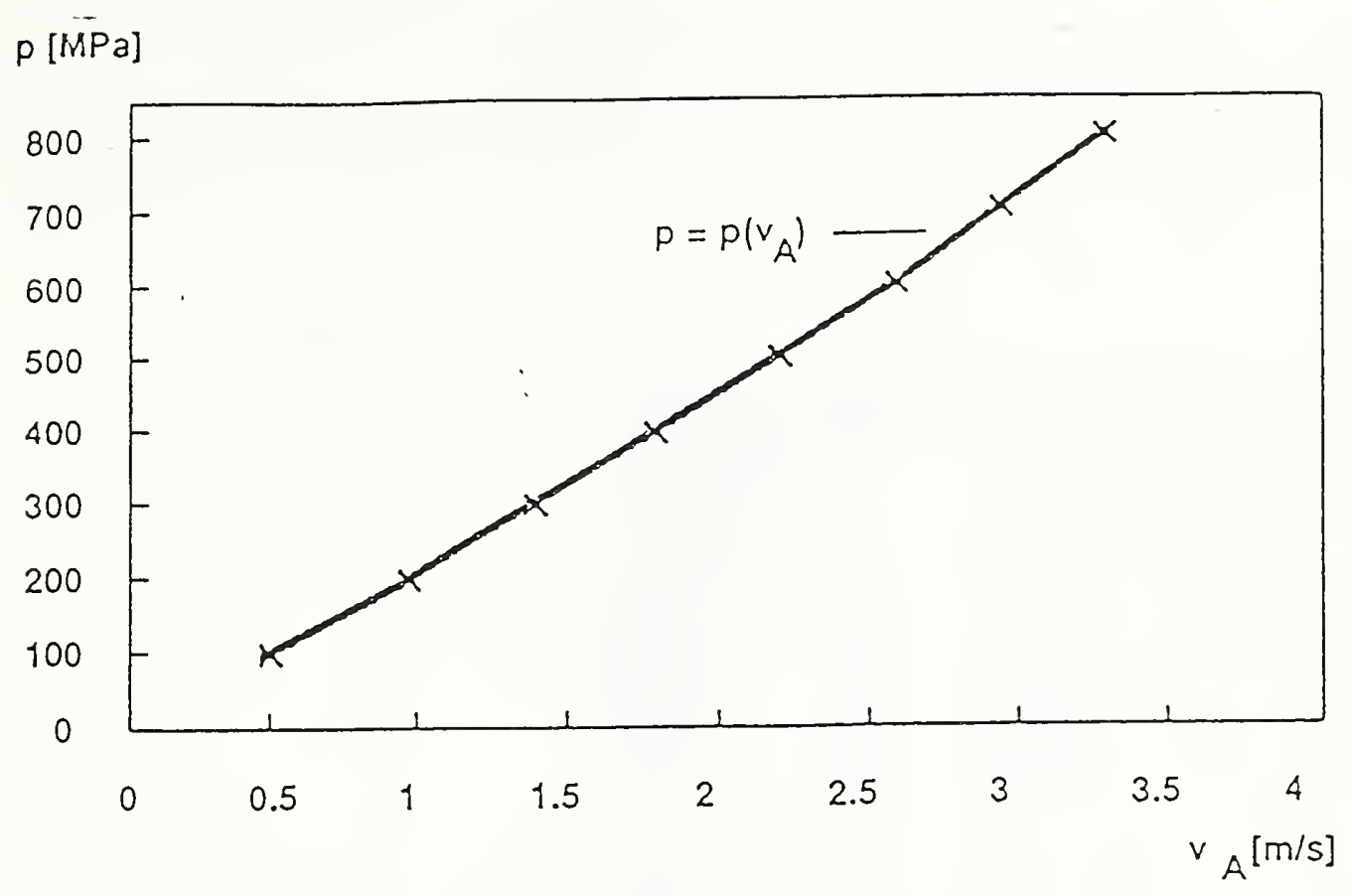

Fig. 20 Dependence pressure - impact velocity

When using the mathematical model for the calibration of the calibrator there are uncertainties at the determination of the $T_{A}$ and $T_{B}$ times of approx.

$+/-5$ - $10 \mu \mathrm{sec}$. Considering the uncertainties of the other influencing magnitudes, such as impact mass weight, piston area and impact velocity, the uncertainty of the mathematical model for the pressure calculation is approx. $+/-3.5 \%$.

Mathematical representation of relation between pressure and falling heights

When the falling heights for the measured pressure values are known, these pairs are used for the polynomial calculation of fourth order according to the least squares method (see table 5).

$$
H(p)=a_{0}+a_{1} \cdot p+a_{2} \cdot p^{2}+a_{3} \cdot p^{3}+a_{4} \cdot p^{4}
$$

Based on this mathematical calculation also other pressure values can be selected. The relevant falling height are automatically calculated by the B621CS Control System from the stored polynomial coefficients $a_{0}, \ldots, a_{4}$. 
Table 5: Coefficients for polynomial of fourth order

\begin{tabular}{|c|c|c|c|c|}
\hline \multirow[t]{2}{*}{ Step } & \multirow[t]{2}{*}{$H$} & \multirow{2}{*}{$\begin{array}{c}P_{\text {eval }} \\
\vdots\end{array}$} & \multirow[t]{2}{*}{$\mathrm{H}_{\text {calc }}$} & $\left(\mathrm{H}-\mathrm{H}_{\text {calc }}\right)$ \\
\hline & & & & $\mathrm{H}$ \\
\hline [bar] & [mm] & [bar] & {$[\mathrm{mm}]$} & [\%] \\
\hline 1000 & 13.58 & 995 & 13.54 & 0.24 \\
\hline 2000 & 48.90 & 2004 & 49.07 & -0.33 \\
\hline 3000 & 102.16 & 2992 & 101.81 & 0.32 \\
\hline 4000 & 171.28 & 4001 & 171.60 & -0.18 \\
\hline 5000 & 254.96 & 4998 & 254.91 & 0.02 \\
\hline 6000 & 352.61 & 6000 & 352.47 & 0.04 \\
\hline 7000 & 464.42 & 7004 & 464.53 & -0.02 \\
\hline 8000 & 591.30 & 8011 & 591.27 & 0.00 \\
\hline
\end{tabular}

$$
\begin{aligned}
& a_{0}=-6913.0721 \cdot 10^{-05} \\
& a_{1}=1667.9072 \cdot 10^{-06} \\
& a_{2}=1281.3057 \cdot 10^{-08} \\
& a_{3}=-7798.4908 \cdot 10^{-13} \\
& a_{4}=3835.7080 \cdot 10^{-17}
\end{aligned}
$$

As the pair of values (pressure versus falling heights) does not lie exactly on the polynomial curve, a systematic error of maximally $+/-0.5 \%$ in the height adjustment (for falling heights $\leq 100 \mathrm{~mm}$ ) is caused. With regard to the peak pressure this represents a relative error of $+1-0.25 \%$. 
One selected reference transducer and three test transducers are mounted on the transfer measuring head into well defined bores with accurate torque (see fig. 21)
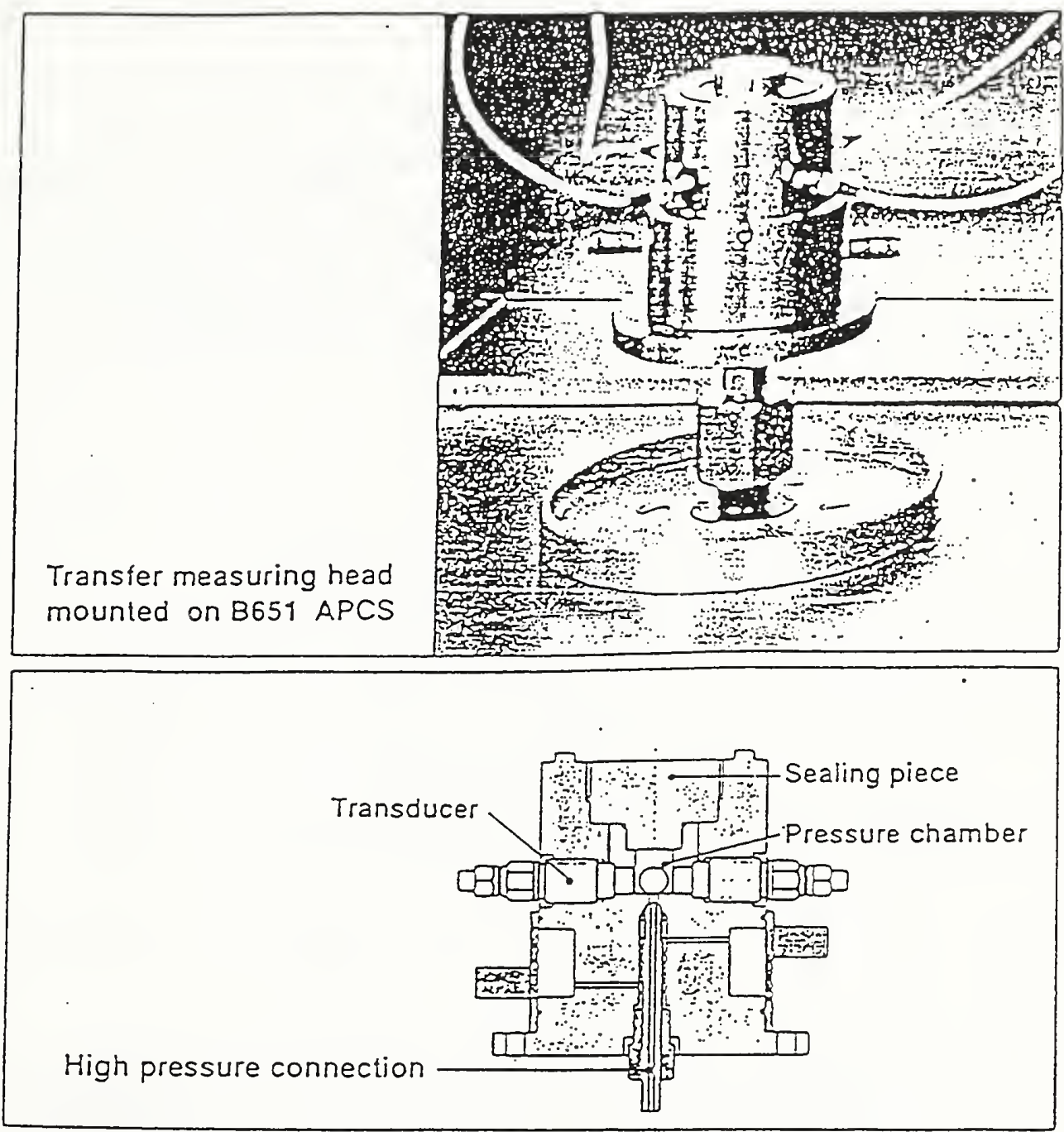

Fig. 21 Set-up of transfer measuring head

Classic static calibration cycles are applied and the data retrieved. The same transducers on the same measuring head are exposed to dynamic calibration pulses, eliminating all minor error influences caused by e.g. bore, adapter, torque etc. When the results of the pressure transducers to be tested and the ones of the reference transducer are compared, they should be in accordance within the uncertainty of the electronic measuring chain (lapprox.+/- $0.2 \%$ ) . 
However, in practice deviations of individual pressure transducers are observed, which lie far beyond this value fig. 22 , see [3].
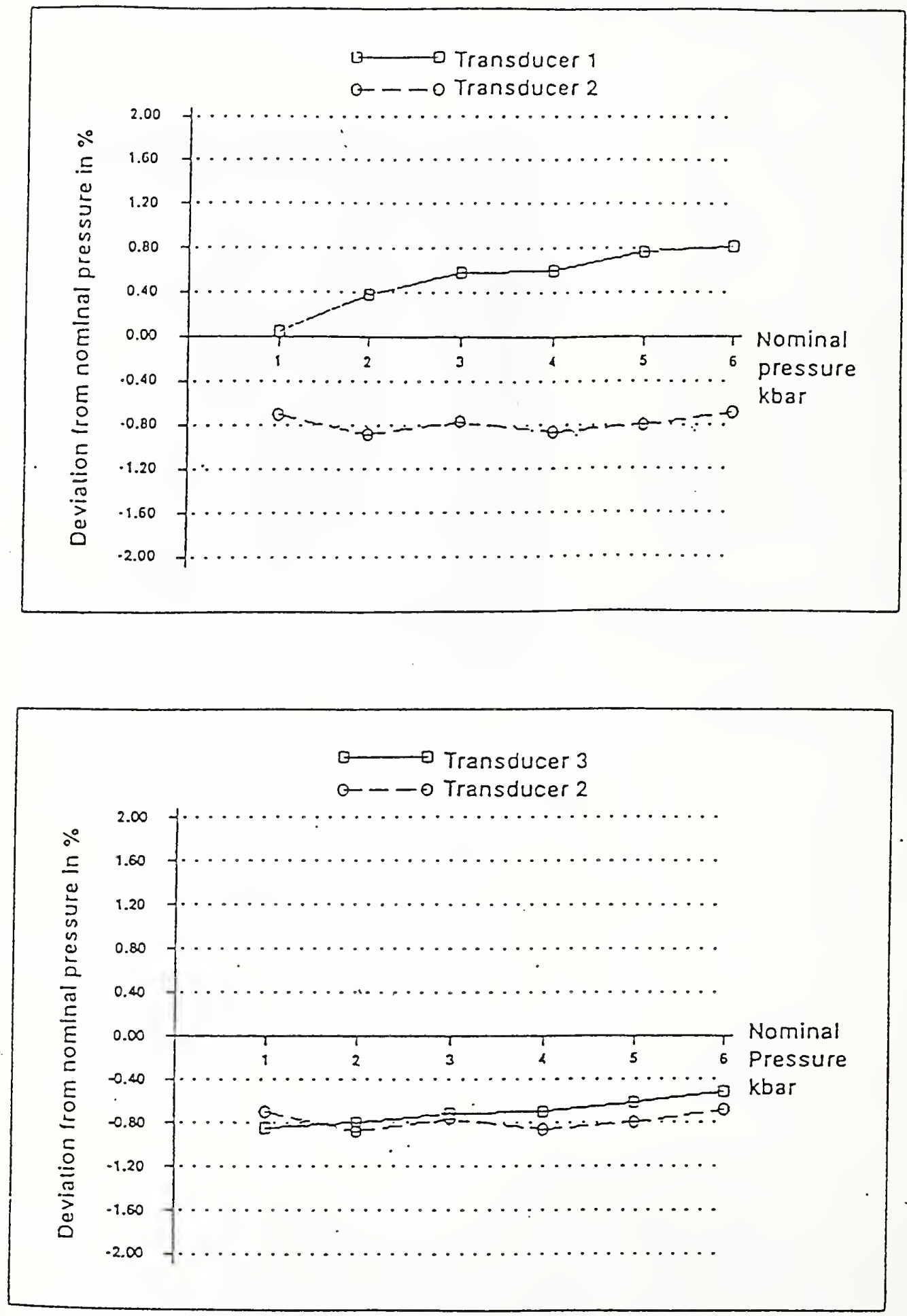

Fig. 22 a) Same pressure - different result b) Same pressure - same result 
In case of further calibrations of pressure transducers e.g. in another measuring head at least one bore for the selected reference pressure transducer has to be available.

This method can also be used to calibrate the calibrator. In that case static and dynamic results have to show a difference of less than $+1-1 \%$. The measured pressure values of the reference transducer(s) are used for computing the polynomial of 4th order which describes the dependency between pressure and falling height.

This method has already been applied at a customer in cooperation with the national Bureau of Standards. The customer defined four specific pressure transducers as reference transducers. With these transducers and the relevant transfer measuring head the static and dynamic calibration was carried out without mounting or dismounting the transducers. All four reference pressure transducers show within $0.8 \%$ the same pressure value at each individual pressure step.

\section{Prospects}

Improvement of the total reproducibility and accuracy

- larger foundation mass

- measure temperature of measuring head with relevant pressure correction

- better mathematical representation of the relation between pressure and falling height (representation with polynomial of 4 th order)

\section{Introduction of an adapter measuring head}

The measuring head is provided with four bores for the adapters, which can be manufactured for any transducer type in such a way that the measuring head volume always remains constant when combining different pressure transducer types. Further advantage is the equal adapter material for calibration (static and dynamic) and in practical use.

\section{Introduction of a fifth pressure transducer bore}

for firmly mounting the reference pressure transducer in the measuring head, this transducer will not be dismounted and will be calibrated statically in the measuring head, if necessary (traceable method). 


\section{Developing measurement methods for obtaining the primary standard}

Starting from the definition of the adiabatic compressibility $\kappa$ (the pressure is generated so quickly that no heat transfer takes place during this time), the pressure generated can be calculated:

integration

$$
\kappa(p)=-1 N \cdot d V / d p \quad\left(V=a \cdot x+V_{0}, d V=A \cdot d x\right)
$$

$$
p(V)=-\int_{0}^{1} 1 / \kappa(p) \cdot A /\left(a \cdot x+V_{0}\right) d x
$$

From this equation it can be seen that when knowing the compressibility of the liquid, the volume of the measuring head and the piston travel, the pressure generated can be calculated. The difficulty for obtaining such primary standard lies in the insufficient accuracy of the presently known compressibility values (at $100-800 \mathrm{MPa}$ ) and in the piston travel which is not easy to measure.

Measuring the acceleration of the impact mass during the impact with

- $\quad$ Brüehl \& Kjaer piezoelectric acceleration transducers

- Polytec laser vibration meter / interferometer

Fig. 23 shows the experimental set-up as applied for measuring the course of acceleration, the velocity and the travel of the impact mass during the impact.
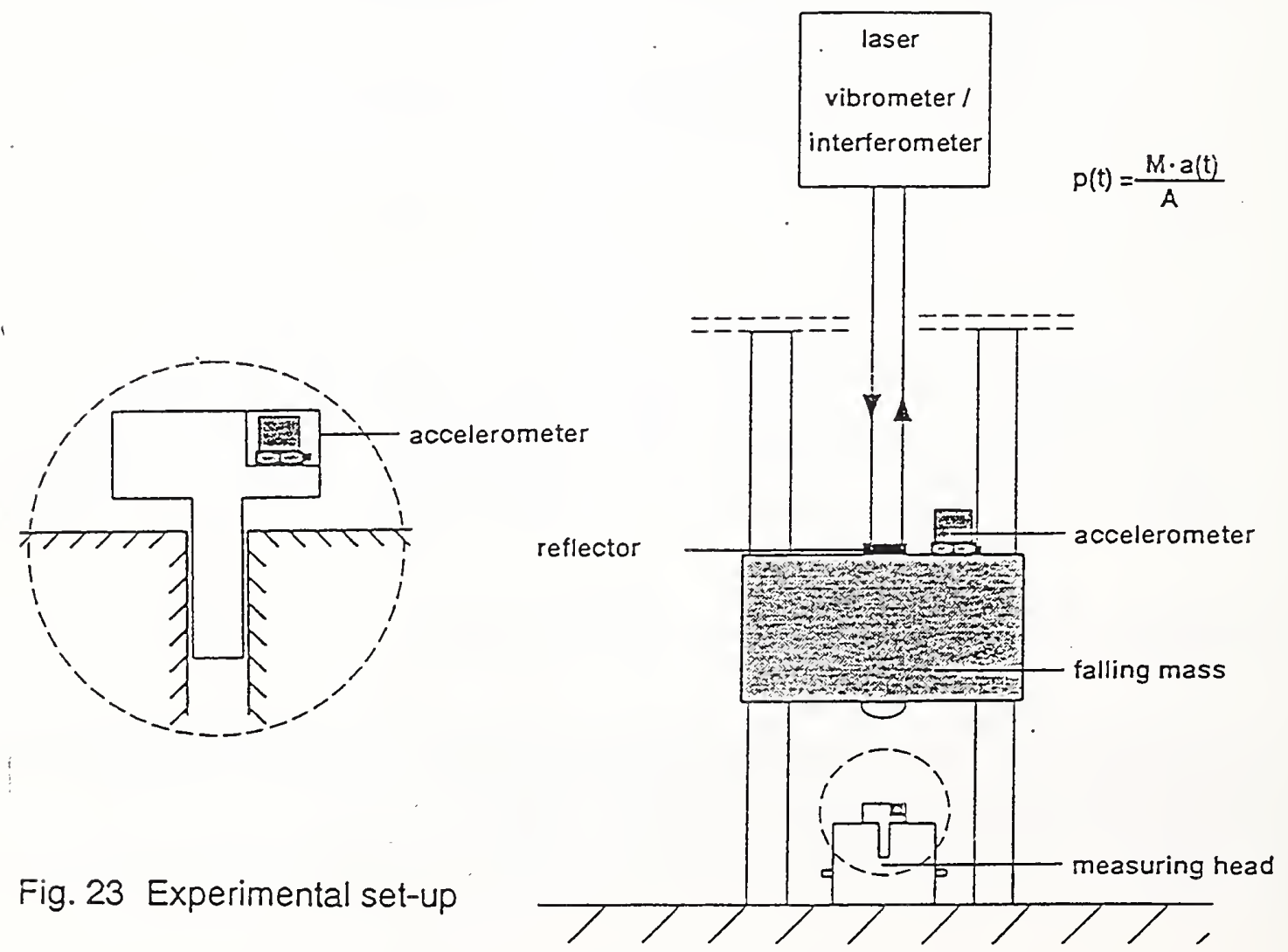

Fig. 23 Experimental set-up 
The courses of velocity and travel measured by the laser vibration meter were used for calculating the acceleration course (see fig. 24). The maximum acceleration from the acceleration measurements was used for further calculations (see fig. 25).

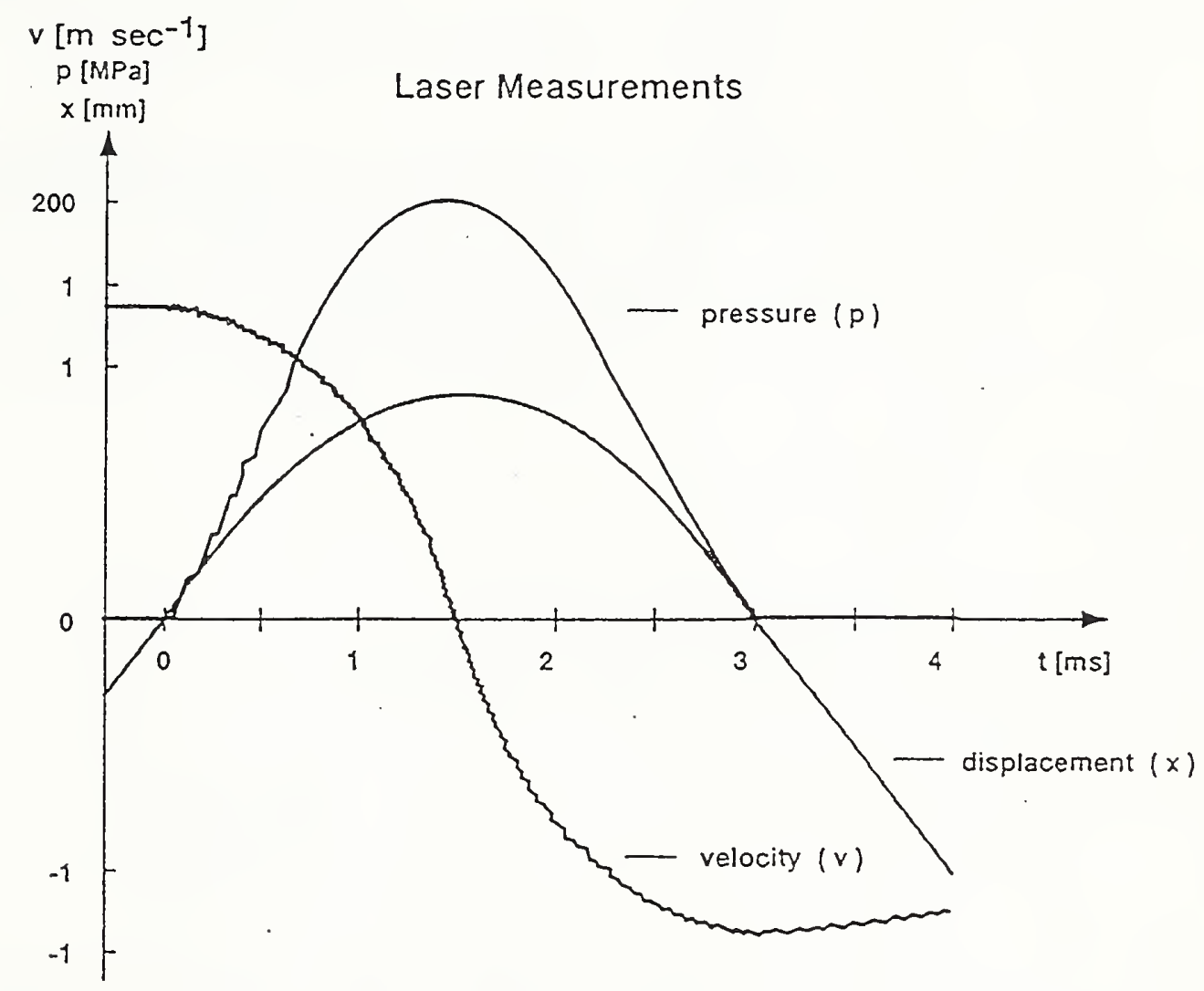

Fig. 24 Results of laser measurements 


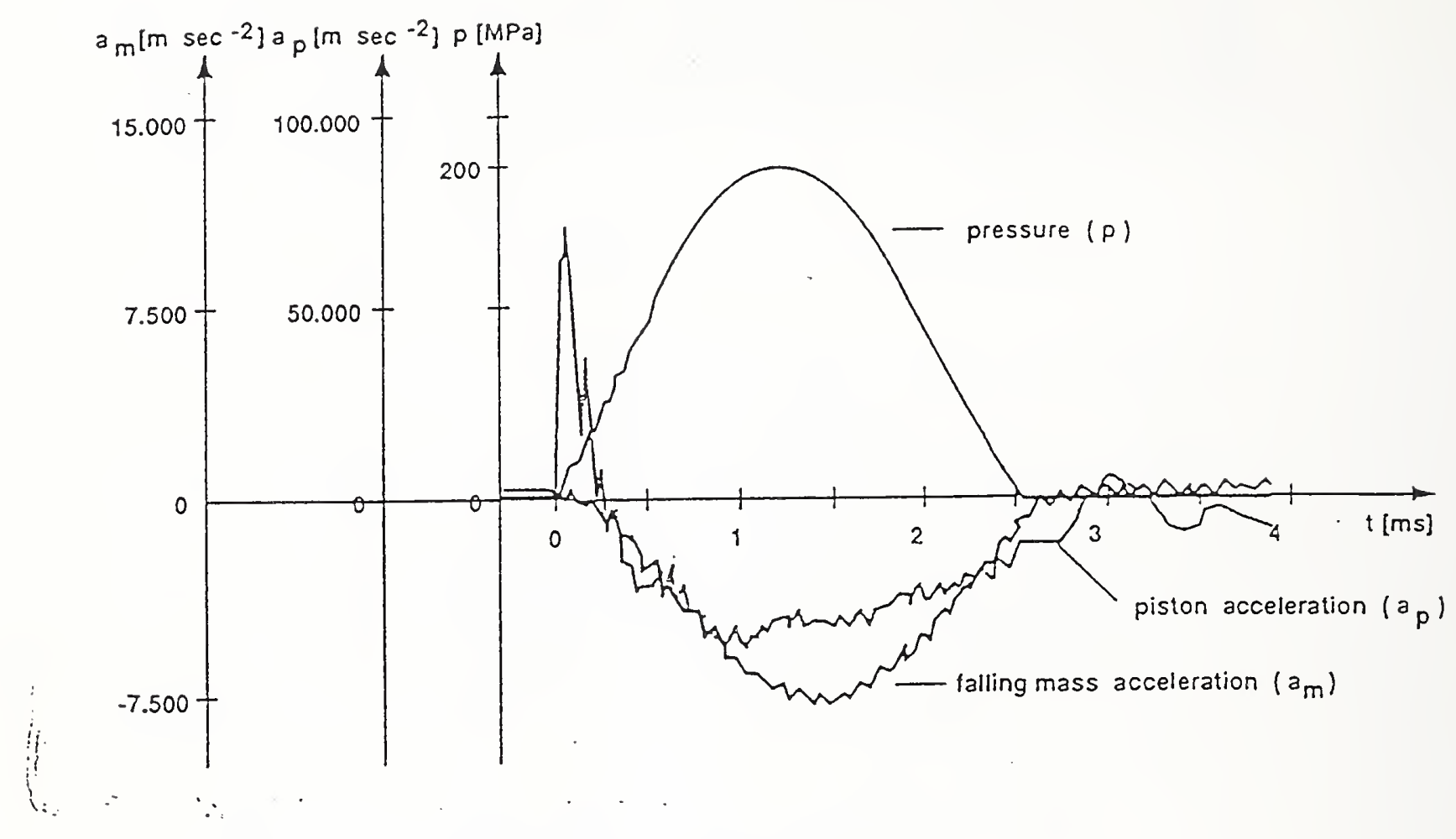

Fig. 25 Results of acceleration measurements

The peak pressure $p_{\max }$ was calculated by using the formula

$$
\begin{array}{lll}
P_{\max }=M^{*} a_{\max } / A & M & \text { impact mass } \\
& \text { a } & \text { acceleration } \\
& \text { A } & \text { piston area }
\end{array}
$$

It corresponds to the peak pressure measured with the pressure transducer in the range of $\leq 4 \%$.

\section{Conclusion}

The error influences of the dynamic pressure calibrator were explained and discussed in detail. Furthermore, the present level of calibration methods for calibrating the pressure calibrator was outlined and prospects for further development work in order to reach primary standard for dynamic pressure were given.

I would like to thank Mr. J. Winkler for his valuable ideas, Mr. R. Kristjan for his technical assistance and Mrs. U. Wutti for the translation of this paper into English and Mr. R. Boschanig for proof-reading, as well as all members of the advertising department for their support. 
References

[1] Dr. Engel, G.

Dynamische Druckmessung mit piezoelektrischen Druckmeßgeräten

AVL List GmbH, Graz, Austria, 1989

[2] Dr. Schwellberger, W.

Der kalte Druckstoß,

AVL List GmbH, Graz, Austria, 1985

[3] Winkler, J.

Quality Improvement in Piezoelectric Pressure Measurements by Applying Advanced Calibration Method

Fourth International AVL Symposium on Ballistic Measurement,

Ochsenboden, Switzerland, 1989 


\title{
WORKSHOP ON THE \\ MEASUREMENT OF TRANSIENT PRESSURE AND TEMPERATURE
}

\author{
22-23 April 1991
}

\author{
National Institut of Standards and Technology \\ Gaithersburg, Mariland
}

MEANS OF DYNAMIC CALIBRATION FOR PRESSURE TRANSDUCERS.

\author{
J.P. DAMION \\ Ecole Nationale Supérieure d'Arts et Métiers \\ Laboratoire de Métrologie Dynamique \\ 151, boulevard de l'Hopital \\ 75013 PARIS \\ FRANCE
}

\section{ABSTRACT}

After a brief review of the different methods permitting the determination of the transfer function of a transducer, this paper presents the means of dynamic calibration of pressure transducers in a gaseous condition, working in the laboratory of dynamic metrology at the Ecole Nationale Supérieure d'Arts et Métiers of Paris, and more especially the shock tubes and the fast opening devices.

The operation of the aperiodic pressure generators is described, and the limits of use in amplitude and frequency are given and also some examples of calibration.

\section{RESUME}

Après un bref résumé des différentes méthodes permettant la détermination de la fonction de transfert d'un capteur, cet article présente les moyens d'étalonnage dynamique des capteurs de pression en milieu gazeux, en service au laboratoire de Métrologie dynamique à l'Ecole Nationale Supérieure d'Arts et Métiers de Paris, et plus spécialement les tubes à choc et les disposititfs à ouverture rapide.

Le fonctionnement des capteurs de pression apériodlque est décrit, et les limites d'utilisation en amplitude et en fréquence sont données ainsi que quelques exemples d'étalonnage. 


\section{INTRODUCTION}

In a static state, a pressure transducer is characterized by its sensitivity which is the ratio of the variation of the output over the variatlon of the input. The sensitivity is practically constant within the range of the transducer.

In a dynamic state, when the input varies with time, keeping the above mentioned ratio, and comparing it with the sensitivity, some differences appear.

A transducer used in a dynamic state cannot be characterized only by its sensitivity, the objective of dynamic calibration is the determination of the transfer function (gain and phase in function of the frequency) able to describe the working of a pressure transducer in its static use or in its dynamic use.

To have access to this transfer function, two important methods are currently used :

- the harmonic method

- the transient method.

To use these two methods, two important types of means are used :

- periodic pressure generators which give a sinusoïdal signal

- aperiodic pressure generators which give a step or a pressure impulse.

\section{METHODS OF DYNAMIC CAUBRATION}

In the harmonic tests, the transducer is submitted to a sinusoidal input pressure of a given amplitude and a given frequency. The ratio of the amplitude of the transducer output over the transducer input gives the gain curve and the phase-angle between the output and the input, the phase curve for a given frequency. Through varying the input frequency point by point, the transfer function can be determined.

In the transient tests, the transducer is submitted to a pressure signal test (impulse - step) and the response is recorded. Then the transfer function $\mathrm{H}(\mathrm{V})$ is calculated point by point and is defined as the ratio of the Fourier transform of the transducer output $S(v)$ over a Fourier transform of the input $E(v)$

$$
H(v)=\frac{S(v)}{E(v)}=\frac{\int_{0}^{\infty} s(t) e^{-j w t} d t}{\int_{0}^{\infty} e(t) e^{-j w t} d t}
$$

\section{MEANS OF DYNAMIC CALUBRATION}

\subsection{Periodic pressure generators}

There are many types of periodic generators which, in most cases, consist of :

- a cavity in which the pressure changes sinusoïdally and where the transducer to be calibrated is placed cavity)

- a pressure modulator (vaniation of volume in the cavity or variation of the mass inside the

- a modulation motor with direct control In frequency (electrodynamic or piezoelectric actuator) or indirect control In frequency vith a cinematic electric motor which converts the circular motion into sinusoïdal linear motion). 
Most periodic generators work in gaseous conditions and their range of operation is in the low pressures and low frequencies. In actual fact, the generated amplitudes are low because the modulation factor must be low in order not to entail too high a distorsion rate. The frequencies are also relatively low for the pressure in the cavity to remain constant (important ratio of the wave length over the main size of the cavity).

Finally, one must bear in mind that there is practically no absolute periodic generator; it is always necessary to use a reference transducer or considered as such.

\subsection{Aperiodic pressure generators}

There are essentially two main types of pressure step generators which work in gaseous conditions :

- the shock tube

- the fast opening device.

The shock tube is the most frequently used means and its application concerns the high frequencies.

The limitation in low frequency is essentlally a function of the duration of the step pressure. The range in amplitude is in low pressure as well as high pressure.

The fast opening device is the extension of the shock tube in the low frequency.

It allows a step function of long duration to be generated, but the rise time is greater than the one met in the shock tube. Its application range goes from quasi-continuous to medium frequency limit which depends a lot on the means.

The amplitude range is the same as with the shock tube.

\subsection{A few remarks on generators}

Periodic pressure generators have a limited use, as the frequency and pressure range are very narrow. Besides, to cover that narrow range, the number of generators to be designed is excessively high. However, user requirements far exceed that application range.

Aperiodic pressure generators with only two main types of equipment cover the high and low pressures anyway and also the high and low frequencies. In spite of the obvious difficulties in carrying out, exploiting and Interpreting the results obtained, the Laboratoire of Métrologie Dynamique of E.N.S.A.M. PARIS is orientated towards the development of aperiodic means which seem to meet user requirements better.

\section{FAST OPENING DEVICE}

\subsection{Operation}

A fast opening device consists of three components :

- a small chamber at pressure P1 where the transducer to be calibrated is placed.

- a large chamber at P2 between the two.

- a system which separates the passage of two chambers and creates a fast opening

The chamber at pressure $\mathrm{P} 1$ being less important than the chamber at pressure $\mathrm{P} 2$, it results that when the opening device is actuated, the pressure in the small chamber changes from $\mathrm{P} 1$ to $\mathrm{P} 2$.

The transducer in the latter is submitted to a step pressure of amplitude P2 - P1. 
Because $\mathrm{P} 2$ can be greater or smaller than $\mathrm{P} 1$, the excitation can be a positive step or a negative step.

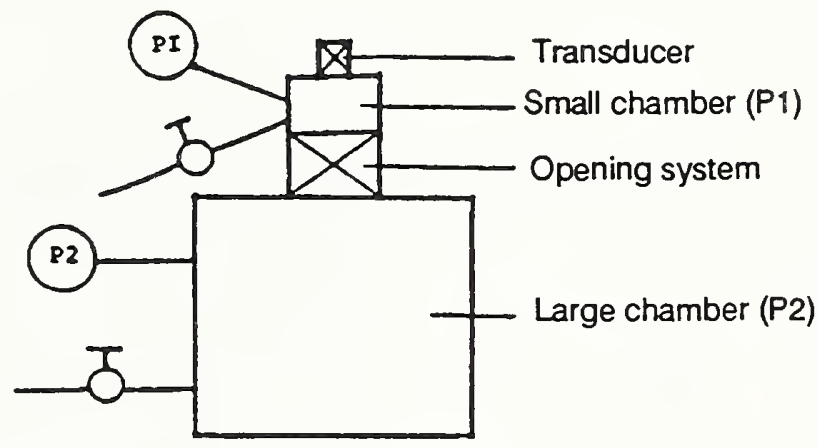

Fig. 1 : Fast opening device

\subsection{Limits of use}

- Amplitude limits

Concerning the high pressures, the fast opening devices are only limited in step generation by the pressure resistance of two chambers and by the available power for controlling of the opening system.

Concerning the low pressures, the fast opening devices can generate lower steps than in the case of the shock tube without too great much difficulty.

- Frequency limits

Concerning the low frequencies, the fast opening devices are only limited by the duration of the recording of the transducer response to be calibrated at a pressure step, as the level time can be as long as we want.

The limits concerning the high frequencies depend especially on the opening system and on the small chamber volum.

In order to determine the frequency limit, it is necessary to measure the "shape" of the transition between pressures $P 1$ and $P 2$ with a reference transducer dynamically tested at the shock tube and to determine the transfer function of the means.

In these conditions, we can effect a calibration using an absolute method (without a reference transducer) or through a comparison method (with a reference transducer). In the latter case, the uncertainties are greater but the limits in frequency are higher.

Figure 2 gives a step response example obtained on a fast opening device and figure 3 the transfer function of this one.

\section{SHOCK TUBE}

\subsection{Qperation}

A shock tube has two chambers with a constant section separated by a membrane. The high pressure chamber (HP) contains the drive gas, and, the low pressure chamber (LP) the working gas. 


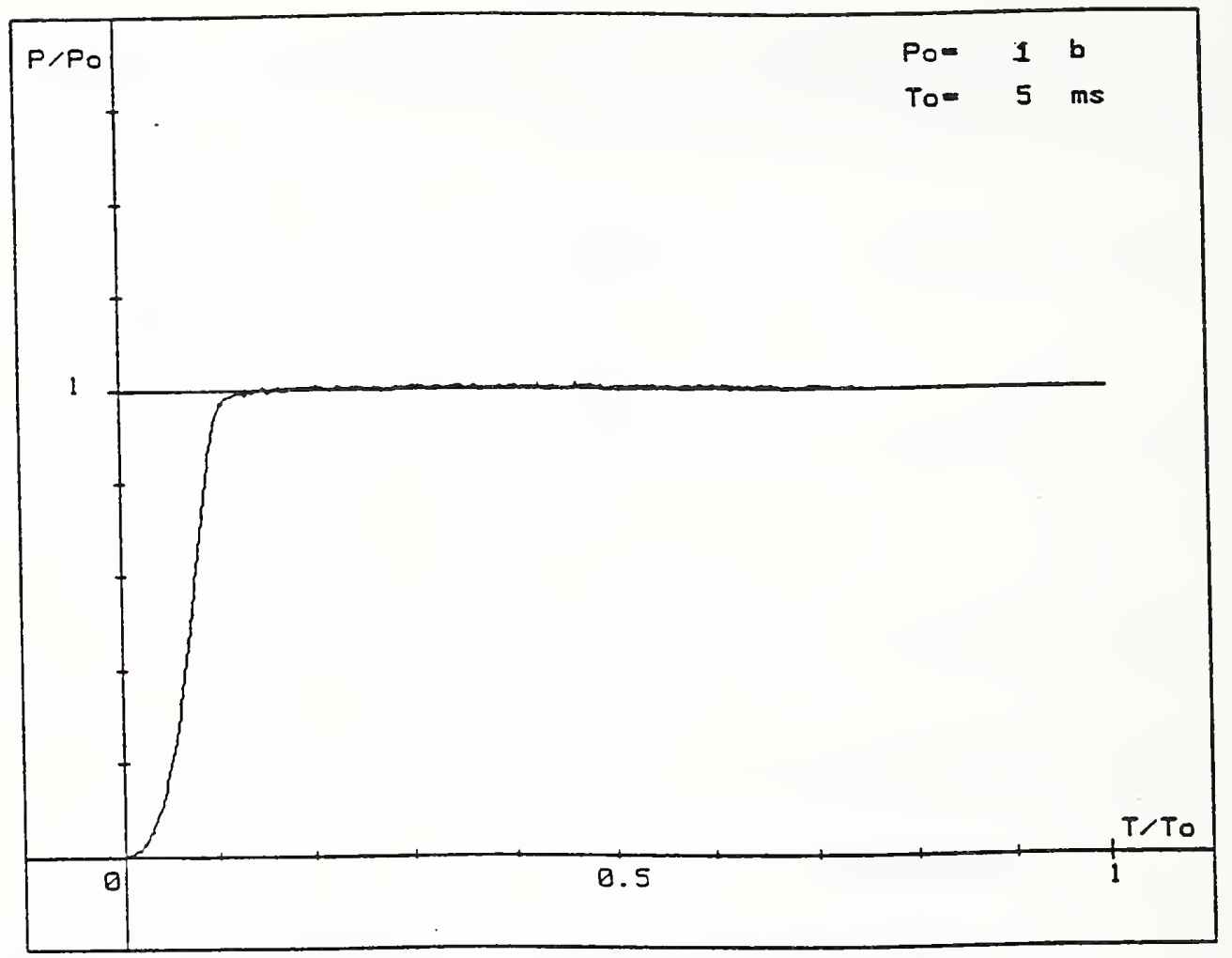

Fig. 2 : Step pressure (Fast opening device DOR100)

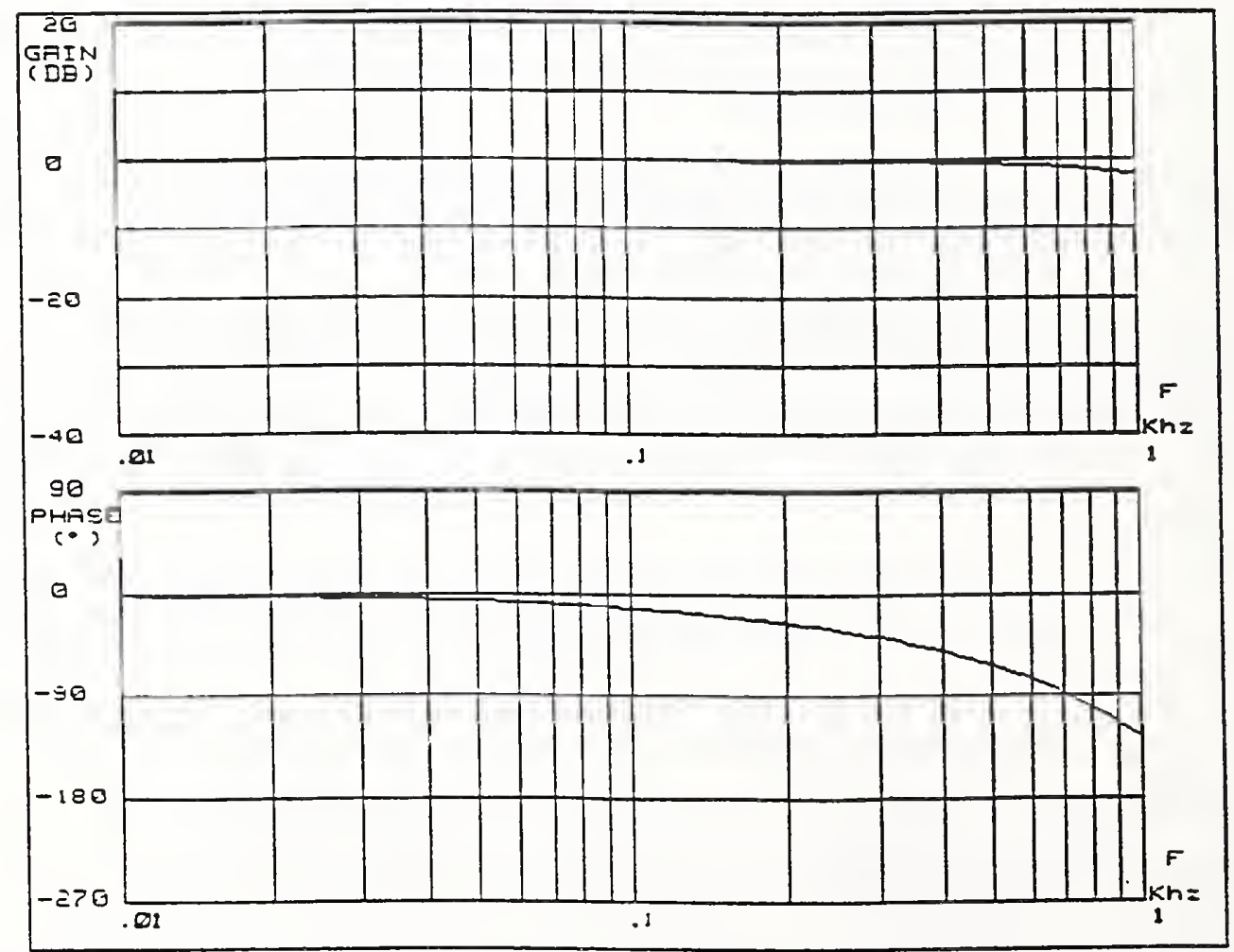

Fig. 3 : Transfer function of DOR 100 
Just before the breaking of the membrarie, the drive gas is in state 4 and the working gas in state 1 . When the membrane breaks at time $t=0$, waves represented in a distance $(x)$ - time $(t)$ diagram propagate in chambers HP et $L P$ (fig. 4).

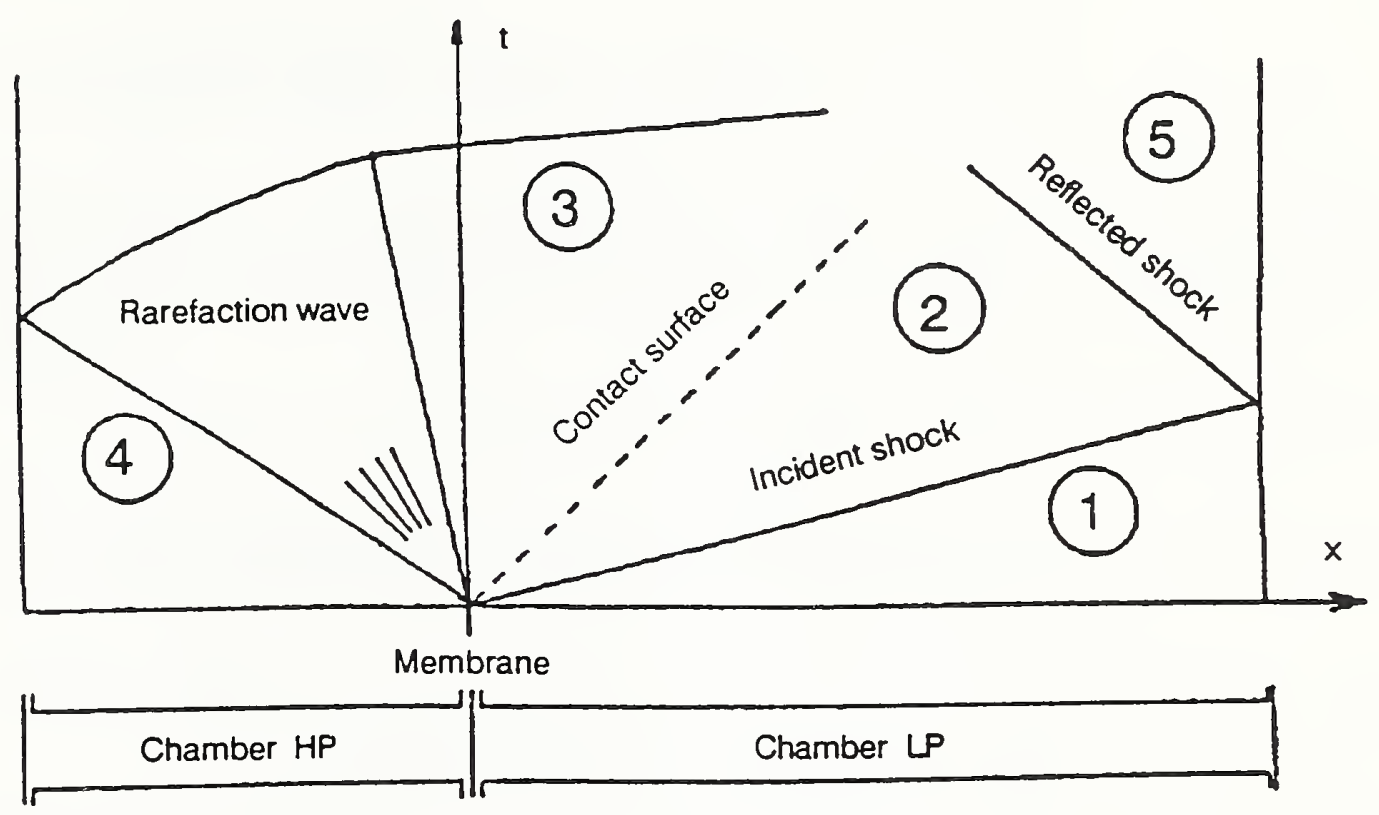

Fig. 4 : Shock tube Distance $(x) /$ Time $(t)$

In chamber HP, a rarefaction wave sends the gas from the state 4 to the state 3 and propagates in chamber HP. In chamber $L P$, a shock wave sends the working gas from state 1 to state 2 and propagates at the bottom of chamber $L P$. The shock wave compresses the gas it is in contact with and imparts it with a given speed. A contact wave is also impelled to the bottom of chamber LP and separates the drive gas from the working gas. The pressures and the speeds are equal on either side of this wave, however, the temperatures are different. When the first rarefaction wave goes to the bottom of chamber HP, it reflects on the solid wall in a rarefaction wave and the pressure falls again behind it. When the shock wave reaches the end of chamber $L P$, it reflects on the solid wall as a shock wave and the gas goes from state 2 to state 5. This shock wave recompresses the gas which is in front of it and stays at rest.

A pressure transducer placed on the wall of chamber BP Is initially at pressure $\mathrm{P} 1$; at the passage of the incident shock, its pressure climbs to P2. For a transducer placed at the bottom of chamber LP, the initial pressure is always $P 1$, but the pressure goes directly to P5 during the reflection of the incident shock.

During a limited time, a transducer placed on the wall (or at the bottom) is submitted, to a step of amplitude P2 - P1 (or P5 - P1); then, the transducer "sees" the continuation of the phenomenon (the reflected shock wave and/or the reflected rarefactlon).

The figure 5 shows an example of the signal obtained at the bottom of the chamber LP for a pressure ratio about 1,3. Figure 6 gives, for this same pressure ratio, the signal lssued from a transducer placed on the wall. 


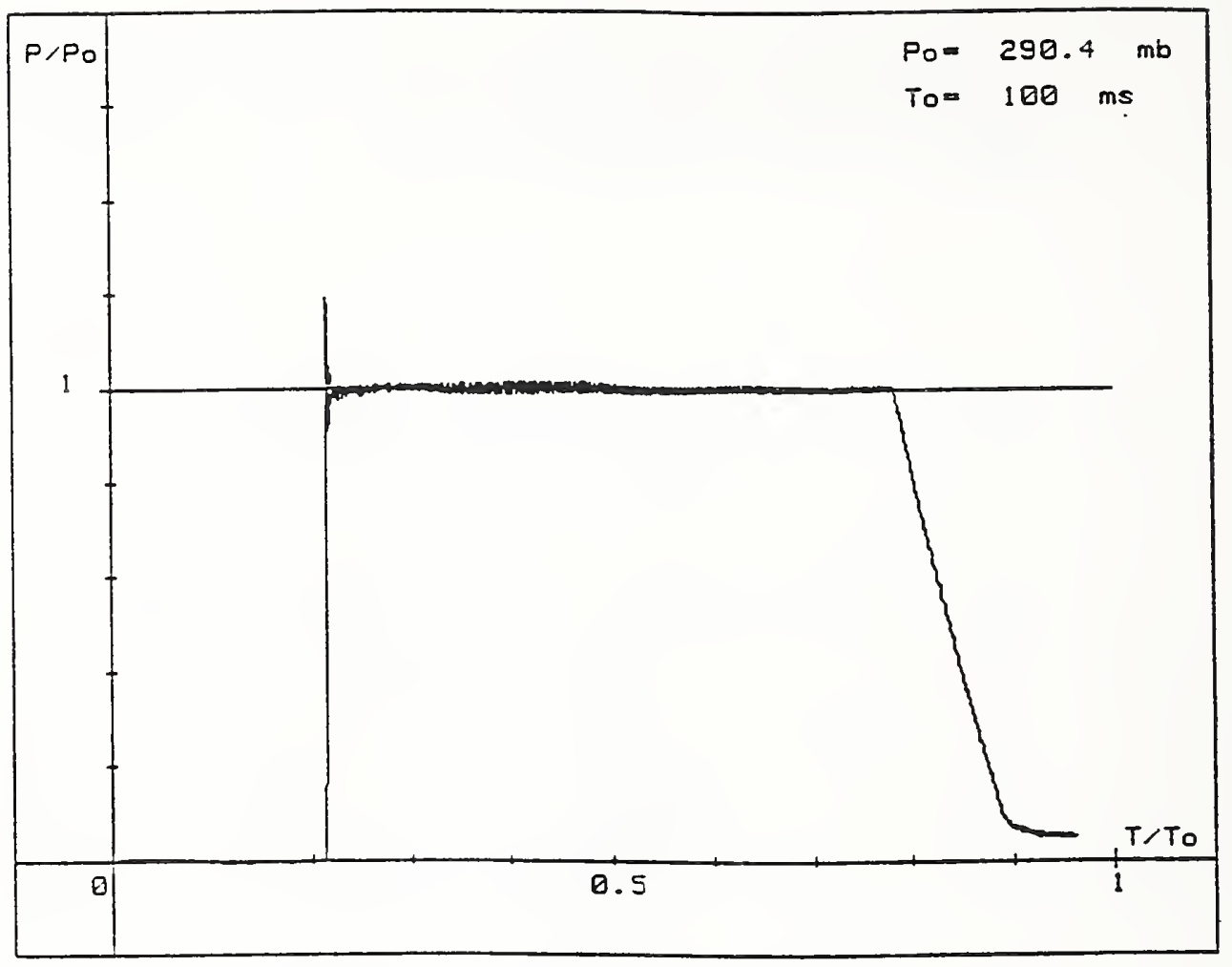

Fig. 5 : Step pressure in the end of shock tube TCR

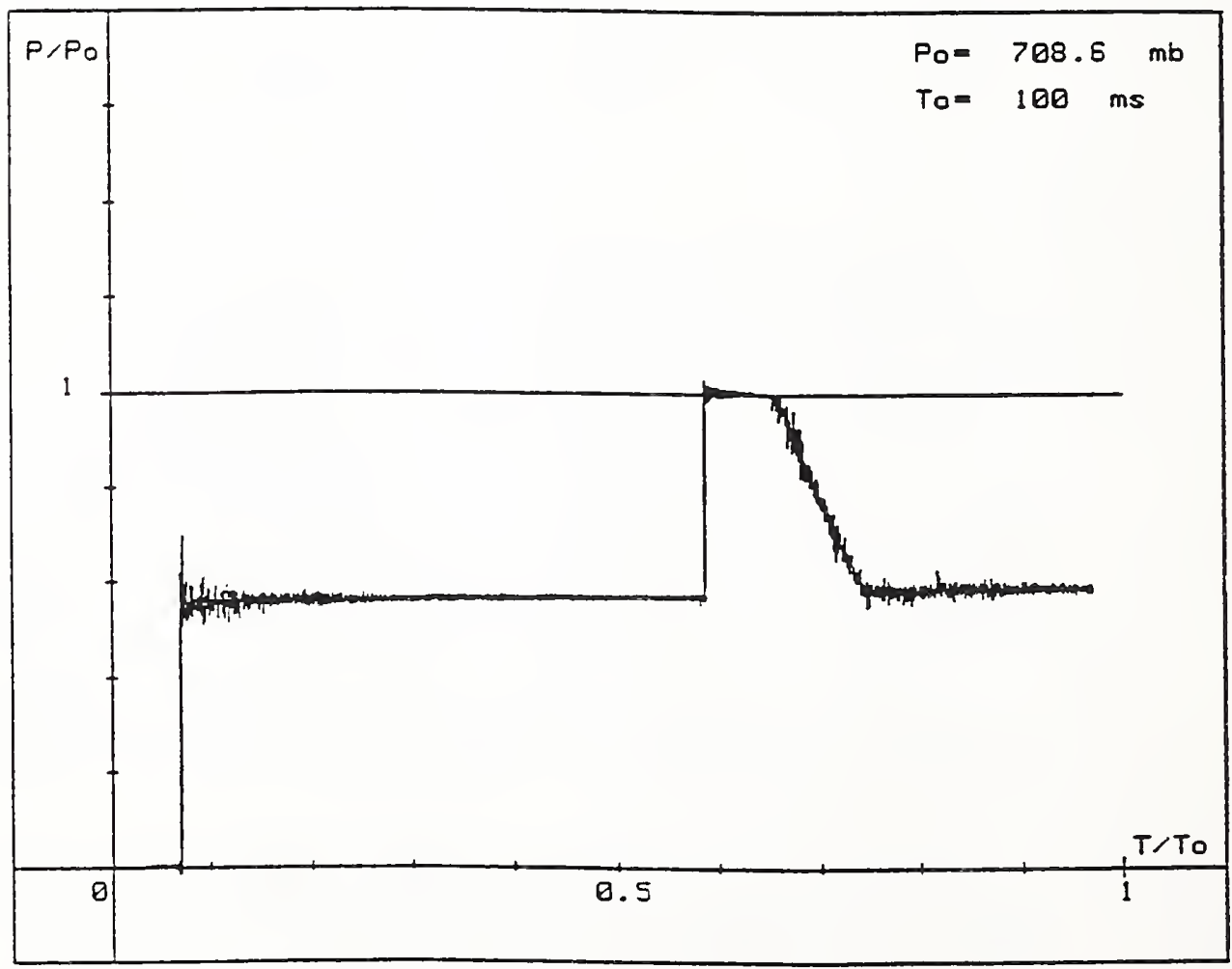

Fig. 6 : Step pressure in the side wall of shock tube TCR 


\subsection{Limits of use}

- Amplitude limits inception :

The amplitude limit (high or low) of the pressure step generated in a shock tube has at

- a purely theoretical limit

- a limit due to disregard of the hypothesis conditions (real operation)

- a technological limit.

The theoretical limit is not a problem because in all cases the real operation of the shock tube sets in before it is reached. As a matter of fact, in order that the flow in the shock tube be kept sufficiently close to the theoretical flow (equation of state in real gas close to the equation of state in perfect gas), the pressure ratio P4/P1 must be as close to the unit as possible. Nevertheless, in order to obtain high amplitudes, it Is necessary to increase the pressure in chamber LP.

With regard to technology, obtaining high amplitudes for creating high pressures is difficult and the safety measures to be taken make its use tricky.

Concerning the low pressures, the difficulties come from the membrane. Its breakage is often unsatisfactory and changes the quality of the pressure step a great deal.

\section{- Frequency limits}

The frequency limits in the dynamic calibration in shock tubes are two-fold:

- the limit in high frequency, which is function of the rise time of the pressure step effectively generated

- the limit in low frequency which depends essentially on the duration of the level of the pressure step.

The position of the calibration section (wall or bottom of the tube BP) is to be taken into account as well as the shape and the physical dimensions of the sensitive surface of the tested transducer.

\section{- High frequency limits}

Theoretically, the "thickness" of a shock wave (the necessary distance for the pressure to go from P1 to P2 or from P1 to P5) is very small (some microns) and moreover, the speed of the shock wave is great, the rise time is generally assumed to be below the microsecond for an imaginery observer placed at a definite point on the wall or at the bottom of the shock tube.

In the case of a calibration at the wall of the shock tube, the actual rise time depends on the speed of the incident shock wave and on the diameter of the transducer.

This limit is the higher as the speed of the incident shock is important and the diameter of the transducer is small.

At the bottom of the shock tube, the rise time is affected nevertheless by the curve of the incident shock wave and by the possible positioning defects of the transducer.

These two phenomena contribute to increasing the effective rise time of the pressure step but in proportions less important than at the wall of the shock tube.

- Low freguency limit

The low frequency limit in a shock tube is due to the finite duration of the pressure level, for 


\section{อเารลกา}

$\lim _{x \rightarrow \infty}$

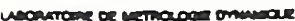

$\begin{array}{lll}\text { FCHIER } & \text { : VF101 } \\ \text { Capteur } & \vdots & \\ \text { Typo } & \vdots & \\ \text { Numéro } & \vdots & \\ \text { K étal. } & 0.02 & \mathrm{mV} / \mathrm{mbar}\end{array}$

ECHELON

Val, init : 3328

Val. palier : 45.2

Temps init : -0.006

Temps fin.

P. init. $: 25000$

Anpl.

mbar

mbar

\section{ETALONNAGE DYNAMIQUE DE CAPTEURS DE PRESSION}

REPONSE EN FREQUENCE

No fréq. $: 100$

Pre. freq $\quad: 100$

Der. fréq : 100

Fitre

:ASEG

$2901-1989$

$10: 44: 23$

$P$ atm. $\quad: 1020$

T amb. : 292

Moyen étal. : TC100

mbar

Uou étal.

ACOUISTION

: 1024

No poirts : NON

Entree : $: 800$

Amp. P.E :2

Temps P.E : 0.002

$m V$

Pér. éch

0.002

Moyen

ADAPTATEUR M 10

Réponse Temporelle

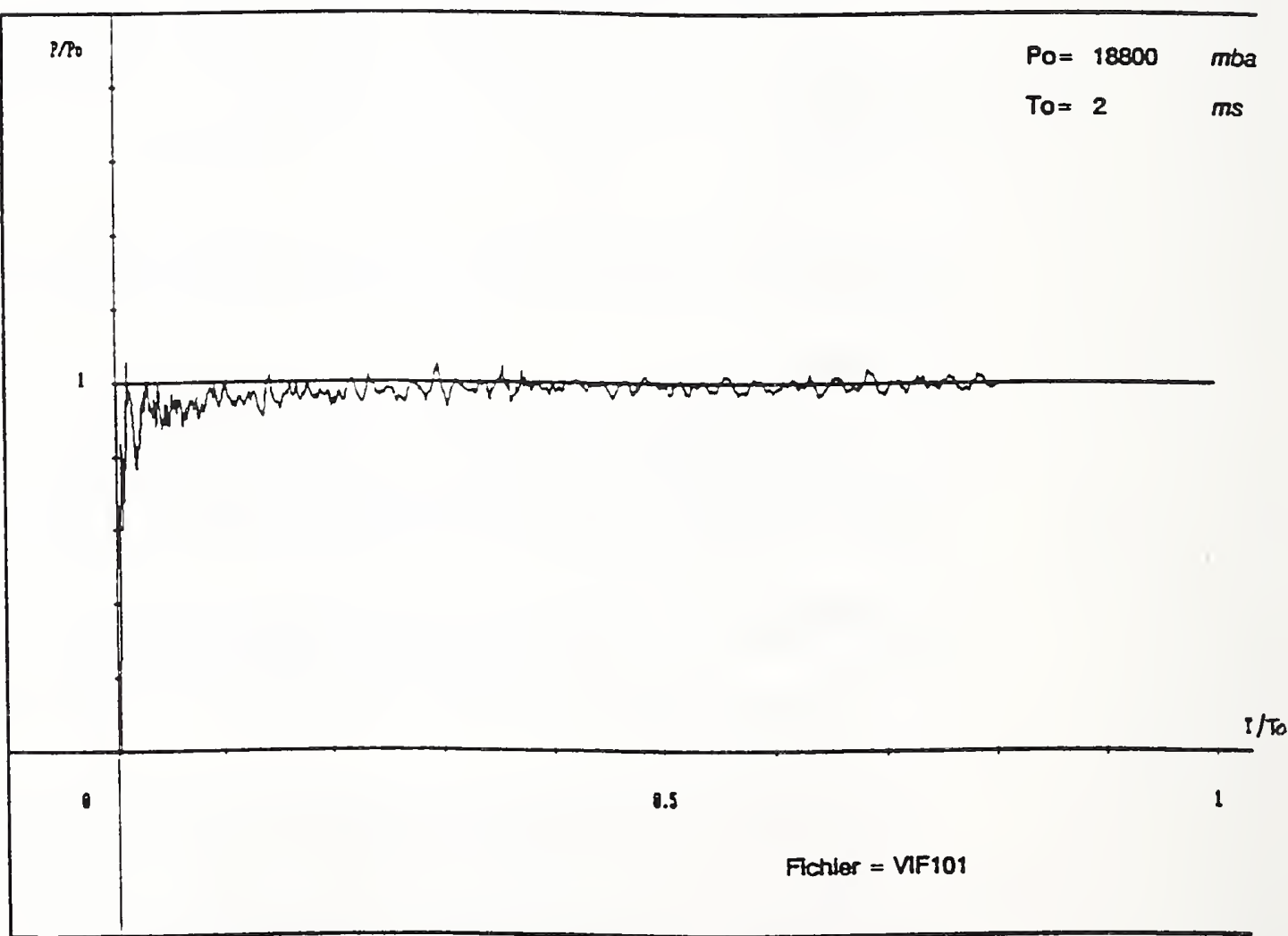

Fig. 7 : Step response of a transducer (TC100) 


\section{ensam}

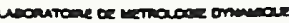

$\longrightarrow$

ETALONNAGE DYNAMIQUE DE CAPTEURS DE PRESSION

$29-01-1989$

$P$ atm. $\quad: 1020 \quad$ mbar

Tamb. :292

Moyen étal. : TC100

K

$\begin{array}{lll}\text { Type } & \vdots & \\ \text { Numero } & \vdots & \\ \text { K étal. } & 0.02 & \mathrm{mV} / \text { moar }\end{array}$

ECHELON

$\begin{array}{lll}\text { Val. Init. } & \vdots & m \mathrm{mV} \\ \text { Val. palier } & \vdots & \mathrm{mV} \\ \text { Temps init } & \vdots & \mathrm{ms} \\ \text { Temps fin. } & \vdots 25000 & \mathrm{~ms} \\ \text { P. init. } & : 18500 & \text { mbar } \\ \text { Ampl. } & & \text { mbar }\end{array}$

\section{REPONSE EN FREQUENCE}

Nb tróq. $: 100$

Pre. fréq $: 100$

Der. tró. :

Fitre

Agorithme
Ulou étal.

\section{ACQUISITION}

Nb points : : 1024

Entróte : : 800

Amp. P.E : 2

Temps P.E : : 0.002

Per ectan : NICOI Moyen

Réponse en Fréquence Moyennée ( 4 fichiers)

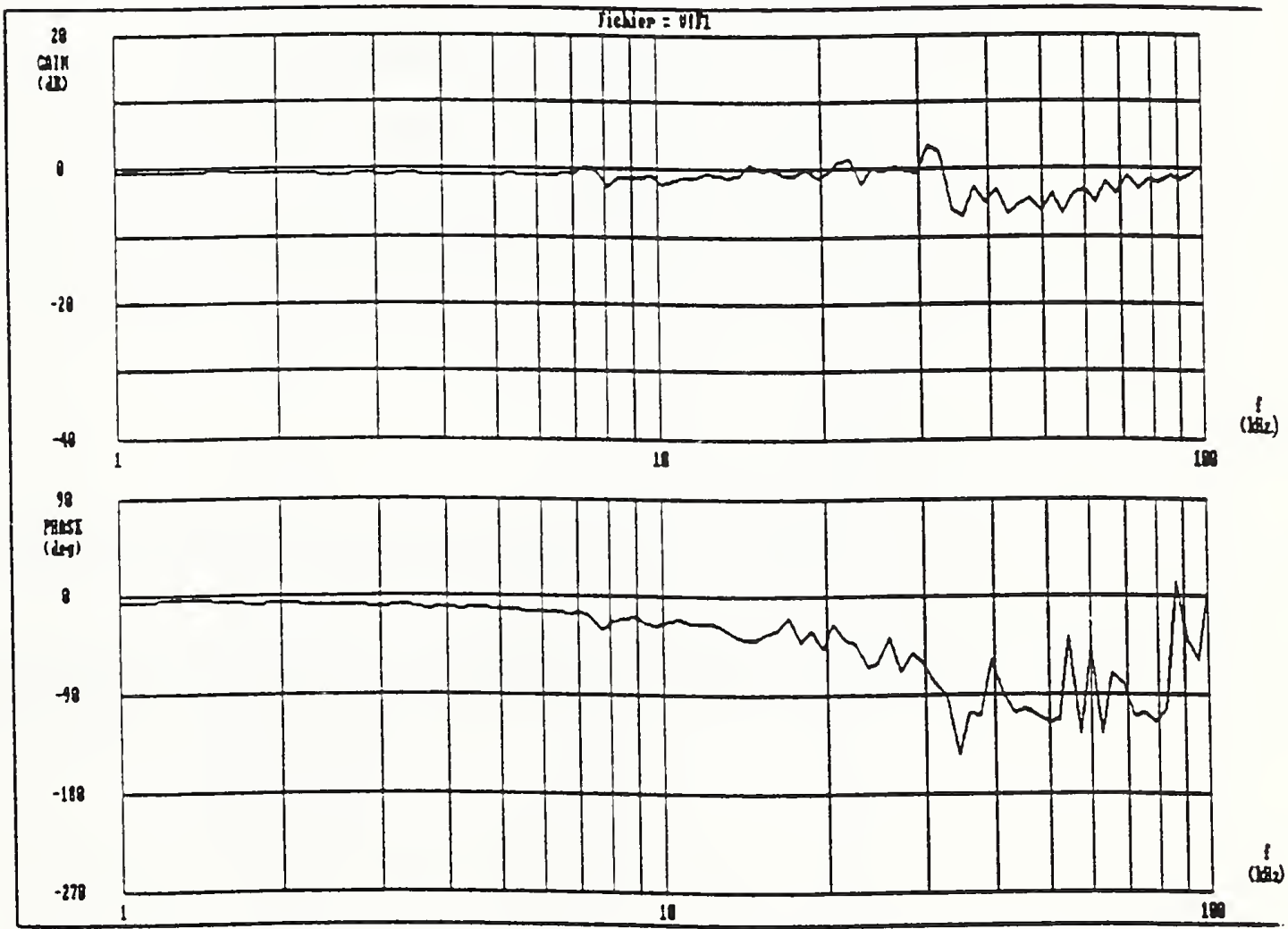

Fig. 8 : Transfer function of a transducer $(\mathrm{TC} 100)$ 
a calibration at the wall or at the bottom of the tube.

The duration of the level, and consequently the low frequency limit, is essentially a function of the length of a shock tube.

Figure 7 gives a step response example of a transducer obtained on shock tube and figure 8 the transfer function of this one.

\section{MEANS OF ENSAM PARIS}

During the last few years, ENSAM PARIS has developed with the help of the Bureau National de Métrologie (BNM) several means in order to satisfy the pressure frequency domain needed by the users.

\section{Shock tubes}

The shock tubes developed by the laboratory are :

- shock tube TC10 which chambers HP and LP are made to work at 10 bars and which enables to generate pressure steps of amplitude from 0,1 to 6 bars.

- shock tube TC100 is able to work in the range of pressure from 5 to 80 bars.

- shock tube TC200 is able to work with a static pressure up to 200 bars.

- shock tube TCR works in low pressure ( 1 to 4 bars) but its step duration is about 50 ms.

\section{Fast opening device}

The fast opening devices developed at the laboratory are :

- fast opening device DOR10 works with a static pressure up to 10 bars and is able to generate step pressure with a rise time of about $4 \mathrm{~ms}$.

- fast opening device DOR100 works at 100 bars and the rise time of the step pressure is about $0.4 \mathrm{~ms}$.

- fast opening device DOR200 have the same performance as DOR100 but the static pressure is up to 200 bars.

\section{CONCLUSION}

The laboratoire de Métrologie Dynamique of E.N.S.A.M. PARIS has developed a coherent equipment to calibrate dynamic pressure transducers in gaseous condition. The whole means are regrouped in the pressure frequency diagram given figure 9.

With shock tubes and fast opening devices, the laboratory can calibrate sensors in pressure range from a few mbars to a few hundred bars and in frequency range from a few $\mathrm{Hz}$ to a few tens $\mathrm{kHz}$. In addition, all the means can be intercompared with the reference shock tube TCR. 


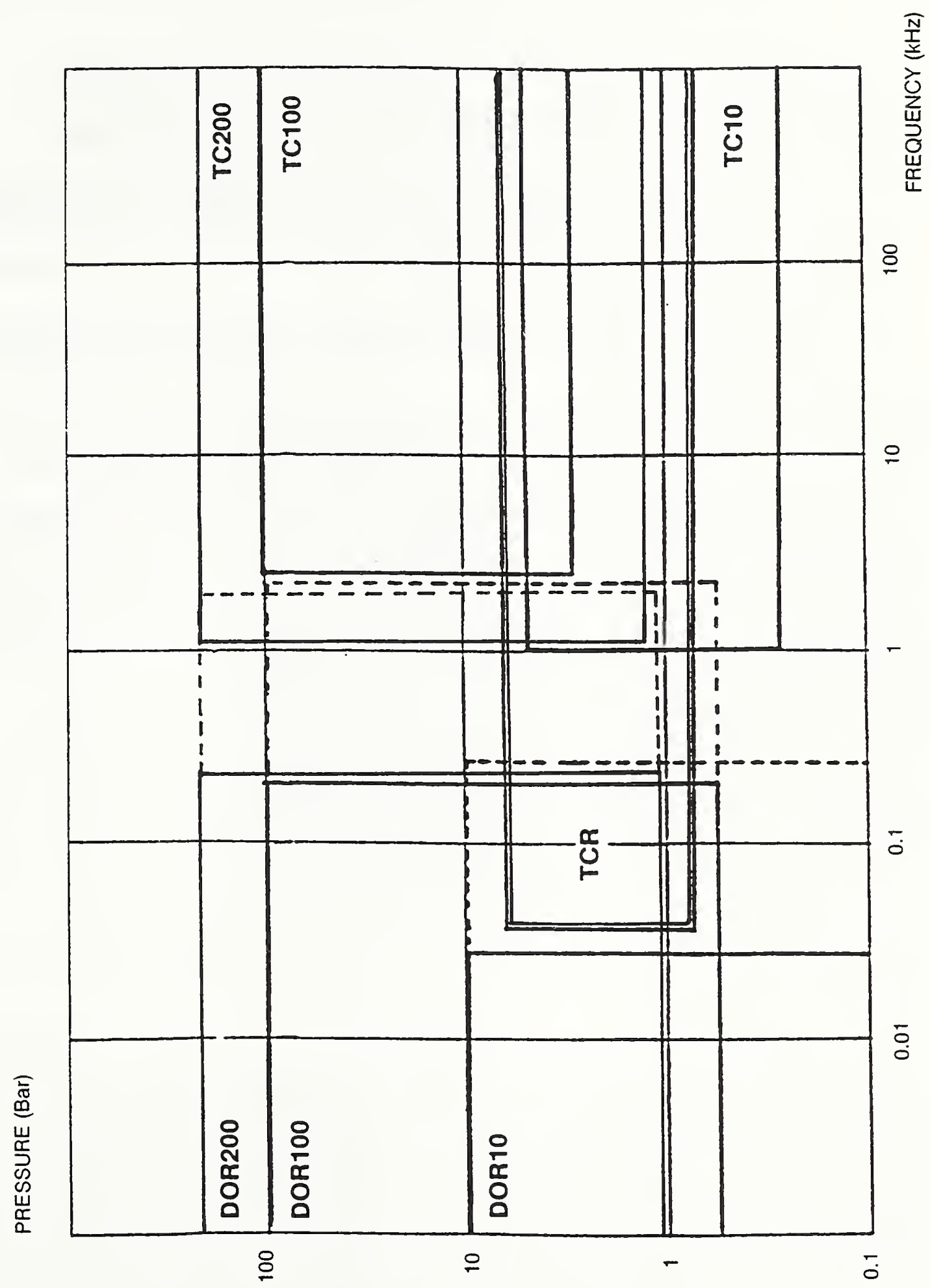

Fig. 9 : Frequency / Pressure Domain of means for dynamic calibration of pressure transducer at ENSAM Paris 


\section{BIBLIOGRAPHY}

- Schweppe, J.L., Eichenberger, LC., Muster, D.F., Michaels, E.L, and Paskusz, G.F., "Methods for the Dynamic Calibration of Pressure Transducers" NBS Monograph 67, December 12, 1963

- ANSI B88.1-1972, A guide for the Dynamic Calibration of Pressure Transducers, American National Standards Institute, 1972

- Paul S. Lederer, "Sensor Handbook for Automatic Test, Monitoring, Diagnostic, and Control Systems Applications to Military Vehicles and Machinery" NBS Special Publication 615, October 1981

- Damion J.P., "Moyens d'étalonnage dynamique des capteurs de pression". Bulletin d'information du Bureau National de Métrologie (B.N.M.) n 30, Octobre 1977

- Damion J.P., "Nouveaux moyens d'étalonnage dynamique des capteurs de pression", Métrologie 85, 16-17-18 Octobre 1985 


\section{A Proposed Dynamic Pressure and Temperature Primary Standard}

Gregory J. Rosasco, Vern E. Bean, and Wilbur S. Hurst

National Institute of Standards and Technology, Gaithersburg, MD 20899
Diatomic gas molecules have a fundamental vibrational motion whose frequency is affected by pressure in a simple way. In addition, these molecules have well defined rotational energy levels whose populations provide a reliable measure of the thermodynamic temperature. Since information concerning the frequency of vibration and the relative populations can be determined by laser spectroscopy, the gas molecules themselves can serve as sensors of pressure and temperature. Through measurements under static conditions, the pressure and temperature dependence of the spectra of selected molecules is now understood. As the time required for the spectroscopic measurement can be reduced to nanoseconds, the diatomic gas molecule is an excellent candidate for a dynamic pressure/temperature primary stardard. The temporal response in this case will be limited by the equilibration time for the molecules to respond to changes in local thermodynamic variables. Preliminary feasibility studies suggest that by using coherent anti-Stokes Raman spectroscopy we will be able to measure dynamic pressure up to $10^{8} \mathrm{~Pa}$ and dynamic temperature up to $1500 \mathrm{~K}$ with an uncertainty of $5 \%$.

Key words: dynamic calibrations; dynamic sources; molecular transducer; nonlinear optical spectroscopy; pressure; primary standard; Raman spectrum; temperature; transducers.

Accepted: October 13, 1989

\section{Introduction}

With modern laser diagnostic techniques, it is possible to characterize the pressure $(P)$ and temperature $(T)$ of a gas at the molecular level. The measurement times for these techniques are such that the response to changes in $T$ and $P$ is limited only by the fundamental relaxation and transport processes of the molecular system. This provides the basis for a new approach to the calibration of transducers used in the measurement of dynamical $P$ and $T$. The essence of dynamic calibrations is the determination of the time dependent response of the transducer, which requires, at a minimum, the application of a stimulus with known time dependence, i.e., a "standard" dynamic source.
If one were to rely on conventional sensors (whose response functions are not a priori known) to characterize the dynamic source, an inescapable circularity emerges from the preceding paragraph. Approaches to solution of this problem have traditionally [1] relied on some form of calculable source. In essence, this is a source some properties of which can be determined from accurate measurements, for example of quasi-static values of $P$ and $T$ and time rate of change of position, and whose time dependent $P$ and $T$ is then derived from an appropriate theoretical prescription, e.g., from hydrodynamics for sound propagation or fluid mechanics for shock waves (with appropriate equa- 
tions of state for isentropic or adiabatic expansions). It must be recognized that every theory relies to some degree on idealizations and that any laboratory realization of a dynamic source is nonideal. Thus, sources of dynamical $P$ and $T$ cannot be accurately known from theory alone; measurement of the "standard" source always is required.

Ideally, in the maintenance of national standards one seeks to relate the measured quantity to a constant of nature, maintained, for example, in the energy levels of an isolated atom or molecule. We are proposing this type of approach for the development of a "standard" source for dynamic $P$ and $T$. The essence of our approach is to combine the very best in calculable generators, fast transducers, and high-speed digital data acquisition systems with a new, fundamental measurement approach. The latter relies on the use of laser-based diagnostic techniques, developed over the past 10 years, to determine the $P$ and $T$ of the dynamic system. The unique characteristics of the optical techniques are:

- $T$ and $P$ are derived from measurement of the optical transitions between the atomic or molecular energy levels of the constituents of the dynamic source, i.e., the atoms or molecules are the fundamental transducers of the local $P$ and $T$ environment

- optical measurements can be accomplished with a single laser pulse of nanosecond duration, with the consequence that the "response time of the transducer" reduces to the equilibration time for the atoms or molecules (in the interaction region) to respond to changes in the local thermodynamic variables

- optical measurements can be accomplished within harsh environments by means of transmitted or reflected laser beams and, for multiple beam techniques, spatial resolution within the source volume can be defined by the regions of overlap of these beams, e.g., $\mathrm{mm}^{3}$ dimensions.

Our approach relies mainly on the use of nonlinear Raman spectroscopies, since these have consistently been shown to provide useful diagnostic spectra in very short times with high spatial resolution[2]. The spectrum determined with these nonlinear Raman approaches is the simplest, best understood, and most highly characterized of any optical diagnostic technique. Comparisons of spectra observed for systems in known (static) states of
$P$ and $T$ with the predictions of theory provide a high degree of certainty in the use of these data for $P$ and $T$ measurement.

The purpose of this paper is to describe the nonlinear Raman optical measurements that can provide the new primary standard for dynamical $P$ and $T$. This description will include information on the $P$ and $T$ dependence of the spectrum and a brief consideration of the important elements of a measurement system which can be applied to a dynamic source. For the purposes of this discussion we do not consider the dynamic source in any detail; however, the information we present is considered applicable to a suitably designed shock tube source. The state-of-the-art in optical diagnostics is now at a point where accurate measurement of such a "standard" dynamic source is possible. Accuracy limits of the order of $5 \%$ for the metrologically significant range of $P$ up to $10^{8} \mathrm{~Pa}$ and $T$ up to $1500 \mathrm{~K}$ appear achievable.

In the following, we begin with an operational description of the use of nonlinear Raman spectroscopy for $P$ and $T$ measurement, drawing from the already established data base on the $T$ and $P$ dependence of observed spectra. We will then outline the elements of a measurement system for a dynamic source. Areas needing significant instrumental development are included in this discussion. Some questions with regard to the $P$ and $T$ dependence of the Raman spectrum which need further fundamental research also are highlighted. The presentation style is intended to be descriptive rather than rigorous; for completeness, more detailed information on the $T$ and $P$ dependence of nonlinear Raman spectra is included in the Appendix.

\section{Nonlinear Raman Optical Diagnostics}

The proposed approach to measurement of the $T$ and $P$ of the dynamic source is coherent anti-Stokes Raman spectroscopy (CARS) [3]. In its most simple realizations this technique uses two lasers, termed the pump and the Stokes beams, whose frequency difference is selected to be in resonance with a pure vibrational transition of a diatomic gas molecule contained in the source medium. The nonlinear interaction of the electric fields of these lasers with the molecules of the source medium generates a third, laser-like beam, termed the anti-Stokes beam, which carries the information about the molecular system, in particular about its $T$ and $P$. 
A useful arrangement of these beams, which provides a high degree of spatial resolution, is shown in figure 1. In this configuration, termed BOXCARS, the interaction volume is defined by the region of overlap of the two pump beams, $k_{0}$ (derived from one laser source), and the Stokes beam, $k_{\mathrm{s}}$. Sample volumes of millimeter and submillimeter dimension are remotely accessible in this arrangement.

The information about the local $T$ and $P$ environment of the molecules in the interaction volume is determined from the spectrum of the generated anti-Stokes beam (designated by $k_{\mathrm{as}}$ in fig. 1). The spectrum is obtained by measuring the power of the anti-Stokes beam as a function of the frequency difference between the pump and Stokes lasers. Considering for the moment a static system, a spectrum can be obtained by measuring this power as we change the frequency difference between a tunable narrowband Stokes-laser and a fixed frequency narrowband pump-laser. Since this is simply a power measurement, we can essentially eliminate the use of traditional spectroscopic in-
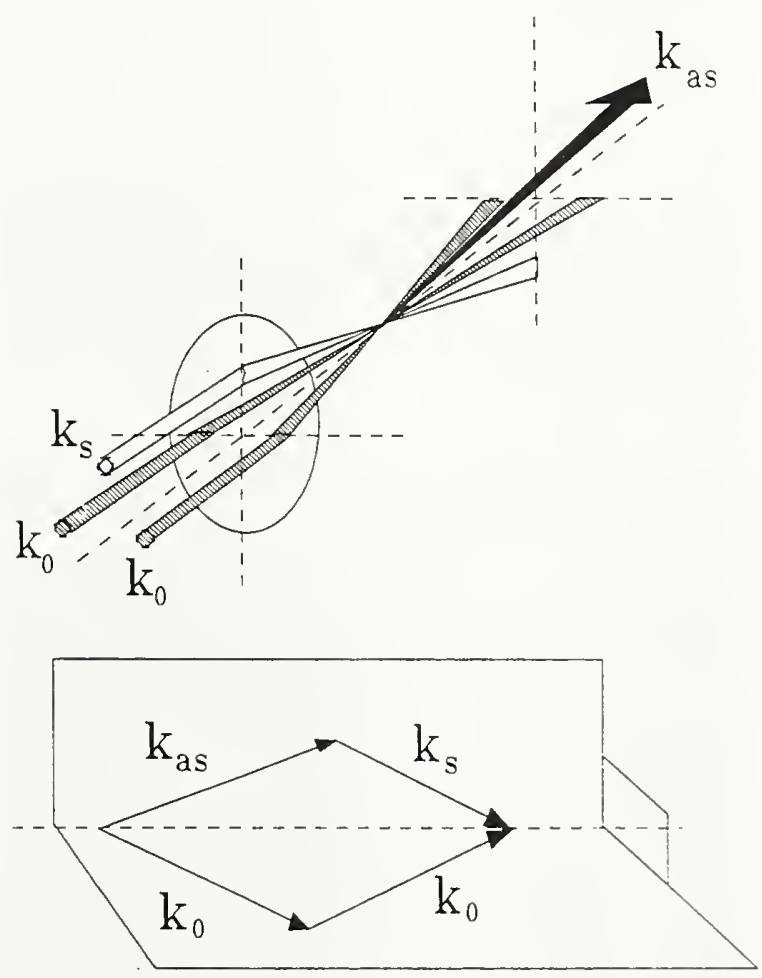

Figure 1. The approximate geometrical arrangement of the pump, (subscript 0), Stokes (subscript s), and generated antiStokes (subscript as) beams in a CARS experiment. The sample region is at the intersection of the crossing beams. The phase matching condition for (folded) BOXCARS [11] is indicated. struments (e.g., prism or grating spectrometers) and retrieve an undistorted measure of the information imparted by the molecular system. The narrowband Stokes and pump laser sources can readily be made of essentially delta-function-like bandwidth for this application.

The special conditions for static systems and narrowband lasers, described in the last paragraph, have been achieved in the laboratory in order to determine the fundamental molecular response, i.e., its spectrum, under known conditions of $T$ and $P$. This has been accomplished for certain ranges of these variables and for a few selected molecular systems [4]. We will illustrate the basics of spectroscopic $T$ and $P$ determinations by describing spectra derived from these studies.

\section{Temperature Dependence of CARS Spectra}

In figure 2 we show CARS spectra of pure $\mathrm{N}_{2}$ as a function of $T$ with the pressure held fixed at 1.0 $\mathrm{atm}$. The horizontal axis is the frequency difference between the pump and Stokes lasers and the vertical axis is the (calculated) power in the anti-Stokes beam (in an unspecified arbitrary unit). These spectra are referred to as vibrational $Q$-branch spectra, because the optical transition involves a change only in the vibrational quantum state (quantum number $v$ ) and no change in rotational state (i.e., no change in the rotational quantum number, $J$ ) [5]. The relevant states and modes of motion are schematically illustrated in figure 3 .

Returning to figure 2, we see that there are many maxima in the power as a function of frequency difference. Each of these arises from a pure vibrational transition which originates in a different rotational state $J$. The vibrational frequency depends, to a small degree, on the rotational state because the rotation of the molecule results in a slight stretching of the bond length producing a small change in the forces binding the molecule and a concomitant change in the vibrational frequency. The vibrational frequency (we use the traditional spectroscopic unit $\mathrm{cm}^{-1}, 1 \mathrm{~cm}^{-1} \simeq 30 \mathrm{GHz}$, in the figure) is approximately $2329.91 \mathrm{~cm}^{-1}$ for the $J=0$ rotationless state; the value decreases approximately according to $0.01738 J(J+1)$, which places the $J=10$ transition at $\simeq 2328.00 \mathrm{~cm}^{-1}$, less than a $0.1 \%$ change. The strength (integrated area) of an individual transition is a function of the population difference between the initial $(v=0, J)$ and the final $(v=1, J)$ states of the transition. This population 


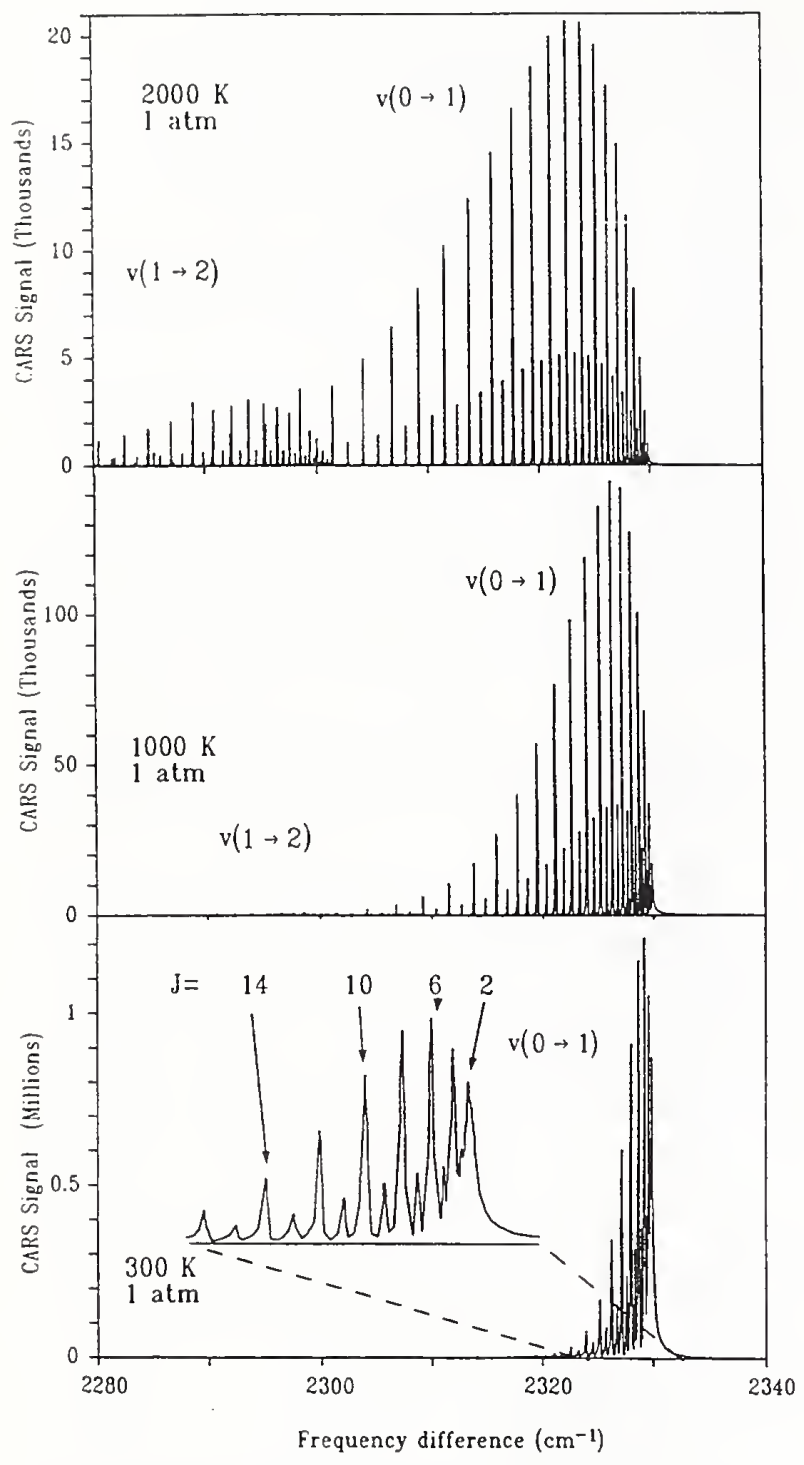

Figure 2. Calculated CARS spectra for the $\mathrm{N}_{2}$ vibrational $Q$ branch as functions of $T$ for fixed $P(=1 \mathrm{~atm})$. The horizontal axis is the frequency difference between the pump and Stokes lasers. The vertical axis is a measure of the CARS power. Although the absolute units of this power are arbitrary, the relative magnitudes as a function of $T$ are accurately represented. Selected transitions and bands of the complete spectrum are indicated. In the bottom panel, the spectral region from $Q(16)$ thru $Q(0)$ is shown on an expanded frequency scale.

dependence in the relative strengths of the transitions as a function of the rotational level, $J$, is the basis for temperature determination, since for systems in thermodynamic equilibrium the state populations are functions only of the temperature. The vibrational $Q$-branch spectrum is very useful for measuring $T$ because there are essentially no corrections to apply in order to relate the strength of a fully resolved transition to the population differene and therefore to the $T[6]$. As is seen in figure 2, the
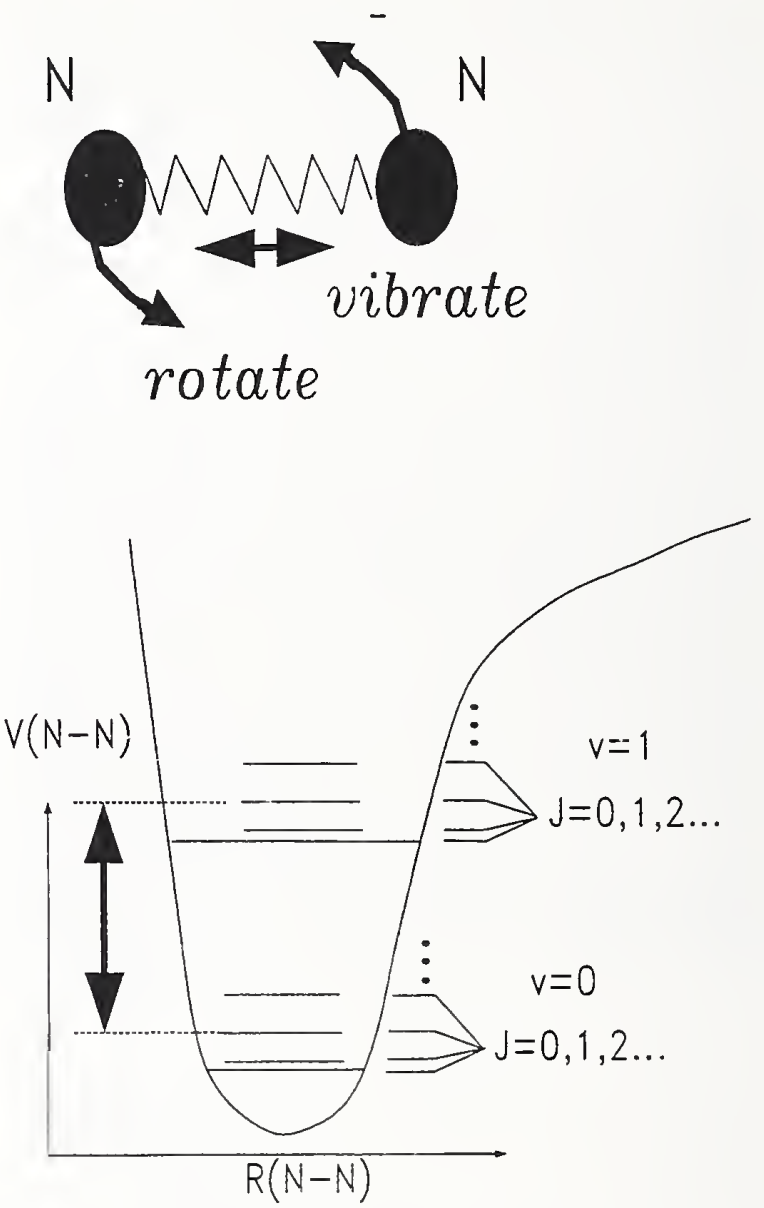

Figure 3. The solid curve schematically represents the potential energy of the ground electronic state versus internuclear separation. Vibrational and rotational energy levels (quantum numbers $\nu$ and $J$, respectively) also are indicated (not to scale). A molecular transition, $Q(2)$, associated with the vibrational $Q$ branch is indicated by the arrow.

transitions for higher- $J$ states increase in strength with increasing $T$; this simply mimics the population shifts to higher energy states associated with increasing $T$.

Strictly speaking, the temperature determined from the relative populations of the rotational levels should be called a "rotational temperature." In like manner, a "vibrational temperature" can be determined from a measurement of the relative populations of the vibrational levels. We observe in the higher- $T$ spectra in figure 2 that there are spectral maxima for transitions labeled $v=1 \rightarrow v=2$. This is a vibrational $Q$ branch which originates in the first excited vibrational level, $v=1$, and terminates in the second excited level, $v=2$. At sufficiently high $T$ there is a significant population in the $v=1$ state and this transition becomes observable. This transition is totally analogous to that discussed above 
which initiated in the vibrational ground state, $v=0$. Comparison of the integrated areas between the $1 \rightarrow 2$ and $0 \rightarrow 1$ transitions gives a measure of the "vibrational temperature." The rotational temperature of the vibrationally excited state also can be determined. The assumption of local thermodynamic equilibrium can thus be tested, since all levels should yield the same thermodynamic temperature in the equilibrium situation.

\section{Pressure Dependence of CARS Spectra}

First we note that the integrated area of the entire spectrum is a function of the number of molecules with which the intersecting laser beams interact. This feature often is used as a means of measuring species concentration in a diagnostic environment $[2,3]$. Each molecular species, e.g., $\mathrm{N}_{2}$, $\mathrm{O}_{2}, \mathrm{CO}, \mathrm{H}_{2}$, etc., has a separate vibrational resonance because the resonant frequency is a sensitive function of the binding forces and the masses of the atoms comprising the molecule. Thus, intercomparison of the areas of these different $Q$ branches can be used as a measure of the relative numbers of each molecule in the sample volume. Because it is very difficult to make accurate measurements of absolute intensity, it has been found that the absolute intensity of a transition is not a good measure of the density or pressure of a sample.

Fortunately, there are good measures of the pressure of a sample which can be recovered from the $Q$-branch spectrum of some diatomics. We illustrate these by spectra of the $Q$ branch of $\mathrm{D}_{2}$, which has been extensively studied in our laboratory. Figure 4 presents CARS spectra calculated from results of these experimental studies. The features of primary interest in this figure are the resonance frequencies and widths of the transitions. Figure 4 illustrates the important fact that these transitions change their width (broaden) and their resonance frequency (shift to lower values) with increasing pressure. In the pressure range shown, this broadening and shifting are linear with pressure. For the $Q(1)$ transition of $\mathrm{D}_{2}$, the broadening rate is $0.0012 \mathrm{~cm}^{-1} / \mathrm{atm}$ (we use the conventional half width at half peak height as our measure of width) whereas the shift rate is $-0.0019 \mathrm{~cm}^{-1} / \mathrm{atm}$, i.e., the line shifts more rapidly than it broadens. We see also that the transitions in the $\mathrm{D}_{2} Q$-branch spectrum remain isolated, non-overlapped, up to $100 \mathrm{~atm}$. We thus identify a very appealing approach to pressure measurement in that it is tied to the measurement of the frequency positions and widths of molecular transitions.

The situation described above for the $\mathrm{D}_{2} Q$ branch should be contrasted with the observations of pressure broadening and shifting in the $Q$ branch of $\mathrm{N}_{2}$. The broadening rates of the $\mathrm{N}_{2}$ transitions are typically 30-40 times larger than that of the $D_{2}$ $Q$ (1) transition. A shifting rate for a $\mathrm{N}_{2}$ transition is typically 10 times smaller than its broadening rate, thereby making accurate frequency determination less certain with increasing pressure. Additionally, we note that the interline spacing in the $\mathrm{N}_{2} Q$ branch is approximately $1 / 60$ th that of $D_{2}$. As a consequence, a transition such as $Q(10)$ would broaden to overlap most of the other transitions shown in figure 2 at a pressure of $100 \mathrm{~atm}$ at room temperature. This overlap of transitions leads to important changes in the appearance of the spectrum which are discussed briefly in the Appendix. At this point it suffices to say that the $\mathrm{N}_{2} Q$ branch involves a complicated spectral distribution function which has the consequence that pressure determinations would generally be less reliable than those derived from the approach based on the $Q$ branch of $\mathrm{D}_{2}$.

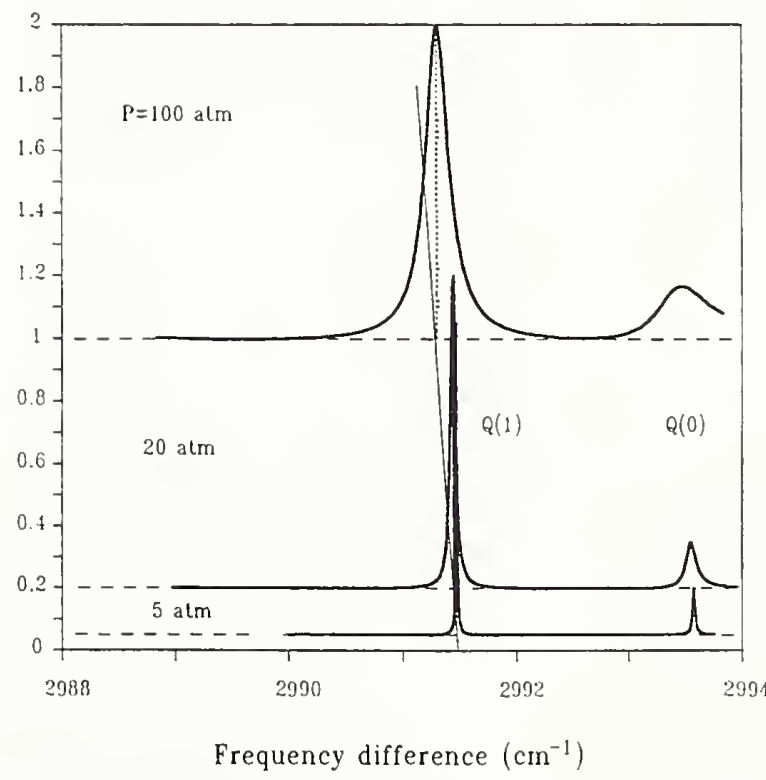

Figure 4. Calculated CARS spectra for the $J=0$ and $J=1$ transitions of the vibrational $Q$ branch of pure $\mathrm{D}_{2}$ at $T=295 \mathrm{~K}$. The zero of intensity for each $P$ is shifted by an amount proportional to $P$. The dotted vertical lines indicate the resonance frequency of the $Q(1)$ line at each $P$. The solid sloping line drawn through the vertical lines is thus an indication of the linear with $P$ shift of the resonance frequency. At $P=0$ the $Q(0)$ line is at 2993.57 $\mathrm{cm}^{-1}$ with the higher $J$ transitions at lower frequencies approximately by the amount $1.056 \mathrm{~J}(J+1)$. 
To this point we have restricted consideration to static systems and very high resolution measurements with narrowband lasers. We have displayed the results of careful measurements under known, static conditions of $T$ and $P$ which demonstrate that temperature can be determined by the measurement of the relative intensities of molecular transitions and that pressure can be determined from the positions and widths of molecular transitions. We turn now to discuss briefly the measurement approach required to characterize a dynamic system.

\section{Single Shot Diagnostic Measurements}

First we point out that we are interested in very rapid measurements, in the range of microseconds or less, in order to characterize the full bandwidth of transducers. Next we note, from the previous discussion, that a relatively large amount of spectral information must be acquired in order to characterize $T$ and $P$. These requirements of time and spectral range (at relatively high resolution) result in the selection of a technique in which all the spectral elements are measured at once, i.e., a single shot measurement. Single shot measurements are the strong suit of nonlinear Raman diagnostics because we can employ pulsed laser sources and obtain high optical power levels which, by virtue of the inherent nonlinear response of these techniques, results in the generation of strong, readily detected, CARS signals.

The most straightforward approach to single shot measurement is to replace the scanned, narrowband Stokes laser, considered above, with a broadband Stokes laser whose frequency width is sufficient to provide the entire Raman spectrum of interest in a single shot. For example, the entire 60 $\mathrm{cm}^{-1}$ region in figure 2 would be measured. We note that a similar spectral bandwidth would be required for temperature measurement via the $D_{2}$ $Q$ branch because of the larger interline spacing in the $\mathrm{D}_{2} Q$ branch, cf. figure 4 . The measurement of the spectrum then requires multiple detectors coupled to an instrument which disperses different frequency components in the anti-Stokes beam to the separate spatial locations of the detectors. This combination of a spectrometer and detector we refer to as a multichannel system.

\section{Instrument Development}

The basic tools for these single shot measurements do exist. The necessary laser systems have been realized in the research laboratory. Adaptation of these to specific diagnostic situations will be required. Similarly, a large variety of spectrometer/detector systems are available and this should allow for reasonably straightforward realization of the specific systems required for our application. Because of the requirements for large spectral ranges and high resolution, noted above, some multiplexing of multichannel systems will be necessary. More details on the instrumentation are discussed in the following.

\subsection{Lasers}

The basic requirements for the laser sources are well within the state-of-the-art of laser technology. The combination of a single-frequency, pulsed Nd:YAG oscillator with a series of amplifiers and a frequency doubling crystal will provide an adequate Raman pump laser. This laser also will serve as a pumping source for amplifying the required broadband dye laser for the Stokes beam. The oscillator for this Stokes laser will require special design, tailored specifically for the transitions selected for optical characterization of the dynamic source.

\subsection{Spectrometer/Detector System}

This system also will be tailored to the transitions chosen. It will be unique in that it will have a high resolution $\left(0.001-0.05 \mathrm{~cm}^{-1}\right)$ system for frequency and linewidth determination along with the more conventional low resolution $\left(0.1-0.5 \mathrm{~cm}^{-1}\right)$ channel for temperature determinations. A high resolution multichannel system has not yet been attempted in diagnostic applications. Our ability to selectively add a component such as $\mathrm{D}_{2}$ to serve as a pressure transducer in the working medium allows us to consider this approach. Incorporating a significant fraction of $\mathrm{N}_{2}$ in this medium allows for a number of independent checks on the temperature and on the state of thermodynamic equilibrium. Depending on the pressure range, we may be able to determine the temperature from the $\mathrm{D}_{2}$ spectrum. An approach under consideration is to simultaneously measure the $Q$ branch of $\mathrm{D}_{2}$ for pressure determination and the pure rotation $S$ branch (transitions within a vibrational state involving a change of +2 in the rotational quantum number only) of $\mathrm{N}_{2}$ to determine the temperature [7].

The discussion in the last few paragraphs indicates one area of the research necessary to develop 
a standard source of dynamic $P$ and $T$. There are additional fundamental studies required to assure accurate knowledge of the $T$ and $P$ dependence of CARS spectra and ultimately to enable the selection of a measurement approach optimized for application to the dynamic source. We turn now to a brief discussion of these.

\section{Fundamental Studies}

The most important fundamental question to be answered in this work is the dependence of the spectra of our specific dynamic system on $T$ and $P$. What is unique and new about the system we propose is that it is by necessity a mixed gas system, for example $\mathrm{D}_{2}$ contained at low concentrations in $\mathrm{N}_{2}$. Except for our recent studies (see ref. [4] and below), there is relatively little work on mixed gas systems. Additionally, we are interested in these systems over quite large ranges of $P$ and $T$.

There are a number of motivations for the selection of a mixed gas system. First, no one molecule presents all the necessary spectral characteristics which allow an adequate characterization of both $T$ and $P$. Of the many single component systems considered, $\mathrm{D}_{2}$ comes closest to fulfilling this requirement. $\mathrm{D}_{2}$ provides a good measure of $P$ because it has very narrow, isolated lines. This characteristic makes it difficult to simultaneously measure the relative strengths of all the $Q$-branch transitions to obtain an accurate measure of $T$. Furthermore, it is much harder (i.e., it takes much longer) to establish thermodynamic equilibrium for $D_{2}$ than it is for $N_{2}$ because of the much larger gaps between the energy levels in $D_{2}$. Large departures from thermodynamic equilibrium can degrade the performance of a dynamic source and the accuracy of the spectroscopic measurements themselves. The use of pure $\mathrm{D}_{2}$ has a number of other limitations; for example, a shock tube dynamic source would be very limited in dynamic range by the use of such a light gas. We note also that the cost of running a pure $\mathrm{D}_{2}$ system would be prohibitive, as also might the potential safety hazards associated with pure $D_{2}$ at high pressures or high temperatures.

The use of a mixed gas system is potentially an advantage for pressure measurements. This statement is derived from our observation that mixed gas systems such as $D_{2}: X$, with $X=H e, A r$, and $N_{2}$, have a larger shift to width ratio than pure $D_{2}$. There remain some fundamental questions in this regard, however, because we have observed line shape asymmetries in the spectrum of $\mathrm{D}_{2}: \mathrm{Ar}$ and $\mathrm{H}_{2}: \mathrm{Ar}[8]$. We also know that these asymmetries are functions of $T$. These observations need to be fully understood in order to reliably calibrate $P$; furthermore, these studies must be extended to the $\mathrm{D}_{2}: \mathrm{N}_{2}$ system. An adequate spectral model and the pertinent molecular parameters must be determined and tested over the complete range of $P$ and $T$ of interest for the dynamic source.

\section{Characterization of the Dynamic Source 8.1 Accuracy Levels in $T$ and $P$}

The accuracy level for dynamic pressure measurements using the proposed optical/molecular approach is estimated to be approximately $5 \%$. This estimate is based on our ability to measure the widths and shifts of the $\mathrm{D}_{2} Q$-branch transitions. For the mixed gas systems proposed as standards, the lines shift at least as rapidly than they broaden. Thus our accuracy goals, imply that we can locate the line center to approximately $1 / 10-1 / 20$ th of the line width and that we can make line-width measurements at approximately the 5\% level. Achieving these measurement accuracies in a single shot experiment is one of the development goals of this work.

Accuracy in temperature measurements depends on our ability to measure the relative intensities of spectral features associated with different molecular quantum states. In practice, this comes down to comparing an observed spectrum to predicted spectra such as those shown in figure 2. In a single shot experiment this comparison must include the effects of a finite-resolution, spectroscopic instrument function. The accuracy of these comparisons for single shot measurements can be of the order of $5 \%[3,4]$. This is the target level of accuracy for temperature measurements on the dynamic source. The simultaneous and independent measurement of pressure will help eliminate systematic errors in either of these measurements.

\subsection{Temporal Evolution}

It is important to recognize that the nonlinear laser diagnostic techniques which allow us to obtain a 10 ns "snap-shot" of the local $T$ and $P$ environment of a molecule, can not easily be extended to provide a continuous-in-time measurement ("movie") of the evolution of $T$ and $P$ in a dynamic source. These techniques do provide a primary standard which can be used to calibrate measure- 
ment approaches which yield good relative measures of $T$ and $P$ with rapid and continuous temporal response. Until this point, we have had no primary standard which could assure the accuracy of these measurements. The primary standard would be used to accurately pin the $P$ and $T$ values at representative points in the temporal evolution of the source during each calibration run. Further, the nonlinear optical techniques would be used to hold the absolute calibration of the source over long periods of use.

\section{Conclusions}

We have described a new approach to the measurement of $T$ and $P$ of a dynamic source. The measurement is based on the fundamental properties of molecules, specifically on the energies and populations of the vibrational and rotational levels of diatomic gas molecules. The nonlinear optical technique, coherent anti-Stokes Raman spectroscopy (CARS), has been proposed as the method for acquiring the information from the molecular system. This technique has the advantages of very rapid (nanosecond) measurement times, small sampling volumes, and a well understood and verified $T$ - and $P$-dependent spectrum. The development of this measurement system to provide a reference standard for dynamic calibrations of $T$ up to $1500 \mathrm{~K}$ and $P$ up to $10^{8} \mathrm{~Pa}$ with a $5 \%$ accuracy appears highly feasible.

\section{Appendix}

\subsection{Raman Scattering}

The Raman spectrum [5] arises from inelastic scattering in which an incident photon of frequency $v_{0}$ is "absorbed," a scattered photon of frequency $v_{0} \pm \Delta v$ is "emitted," and the molecule undergoes a transition among its internal states. The energy difference between the scattered and incident photons, $h \Delta v$, is equal to that between the internal states of the scattering molecule. More concisely, Raman scattering, as we will consider it in this discussion, will refer to a two-photon transition originating in the ground electronic state, with intermediate states being far off-resonance, onephoton-allowed electronic transitions. The initial and final states of this transition are molecular vibration-rotation levels in the ground electronic state.

\subsection{Nonlinear Optics}

The fact that Raman scattering is a two-photon transition makes it essentially a nonlinear optical phenomenon. Many nonlinear Raman spectroscopy techniques simply involve impressing (on the molecules) optical fields corresponding to the two photons of the Raman transition. In the following we will briefly discuss Raman scattering in the context of nonlinear optics [3,9]. In this theory the response of a macroscopic system to an applied electromagnetic field, $E$, is written in a form such as

$$
\Pi \propto \chi^{(1)} E+\chi^{(2)} E: E+\chi^{(3)} E: E: E+\ldots
$$

where $\Pi$ is the polarization density induced by the action of the fields and $\chi^{(n)}$ is the $n$th order susceptibility (a tensor of rank $n+1$ ). The relarionship in eq (1) is intended to indicate that we are concerned with vector quantities and that the response of the system involves, in general, all orders of the electric fields. The electromagnetic fields and polarization densities appearing in this expression typically are assumed to be monochromatic (angular frequency $\omega$ ) plane waves (wave vector $k$ ) with the representation

$$
E=\sum_{i} 1 / 2 E\left(\omega_{i}\right) \exp \left(k_{i} \cdot r-\omega_{i} t\right)+\text { c.c. }
$$

(c.c. denotes complex conjugate) with

$$
k_{i}=\frac{n_{i} \omega_{i}}{c}
$$

where $n_{i}$ is the index of refraction and $c$ the speed of light. With this representation of the fields and polarization, the susceptibilities are written as functions of the frequencies of the associated monochromatic components of the field. Nonlinear Raman effects occur when the frequency differences and electric field directions of the applied fields can drive a Raman transition.

\subsection{Coherent Anti-Stokes Raman Spectroscopy}

In particular, the nonlinear Raman effect known as coherent anti-Stokes Raman spectroscopy (CARS) arises from the 3rd-order terms with electric field components $E_{j}\left(\omega_{0}\right), E_{k}\left(\omega_{0}\right)$, and $\left[E_{l}\left(\omega_{a s}\right)\right]^{*}$ (the $j, k, l$ are spatial indices and the "** denotes complex conjugate) such that a polarization density at frequency $\omega_{\text {as }}$ with wave vector $K_{\text {as }}$ is generated with the conditions 


$$
\omega_{\mathrm{as}}=2 \omega_{0}-\omega_{\mathrm{s}}
$$

and

$$
K_{\mathrm{as}}=2 k_{0}-k_{\mathrm{s}}
$$

These equations are essentially statements of conservation of energy and momentum. The latter condition indicates that a phase matching geometry must be established between the incident "pump" field $\left(\omega_{0}\right)$ and the incident "Stokes" field $\left(\omega_{s}\right)$ in order to generate a signal beam, the anti-Stokes component $\left(\omega_{a s}\right)$. The corresponding susceptibility has 2-photon, Raman resonances when

$$
\omega_{0}-\omega_{\mathrm{s}}=\Delta \omega_{\text {molecular }}
$$

with $\Delta \omega_{\text {molecular }}$ being a molecular transition angular frequency $\left(2 \pi \Delta \nu=\Delta \omega_{\text {molecular }}\right)$.

\subsection{Raman $Q$-Branch Resonance}

For example, the fundamental (i.e., $v=0 \rightarrow v=1$ ) vibrational $Q$ branch is composed of all transitions from the ground to first excited vibrational level without change in rotational quantum number; the molecular transition frequency in this case is

$$
\Delta \omega_{\text {molecular }}=\hbar^{-1}[E(v=1, J)-E(v=0, J)] .
$$

This relation yields a multiple line spectrum for the $Q$ branch because the rotational energy level spacing changes with the vibrational state. The transition frequencies for the vibrational $Q$-branch of a simple diatomic molecule such as $\mathrm{N}_{2}, \mathrm{CO}$, or $\mathrm{D}_{2}$ can be expressed in terms of familiar molecular constants $[5]$ as

$$
\nu(J) \simeq v_{c}-\alpha_{e} J(J+1)+\beta_{e} J^{2}(J+1)^{2}
$$

with $v_{0}$ the frequency of the pure vibrational transition between the rotationless $(J=0)$ states and with the $\alpha_{\mathrm{e}}$ and $\beta_{\mathrm{e}}$ expressing, respectively, the change in the moment of inertia and (lowest order) centrifugal distortion with vibrational state. In keeping with convention, we will express frequency in the spectroscopic unit the wavenumber, $\mathrm{cm}^{-1}$, which is the frequency in $\mathrm{Hz}$ divided by the speed of light in $\mathrm{cm} / \mathrm{s}[\nu / c=\omega /(2 \pi c)]$.

\subsection{CARS Susceptibility}

The CARS susceptibility resonance for the isotropic vibrational $Q$ branch with laser, Stokes, and anti-Stokes electric field vectors in parallel orientations is defined by the following relations [10]

$$
\begin{aligned}
& \Pi_{1}\left(\omega_{\mathrm{as}}\right)=3 \chi_{1111}^{(3)}\left(-\omega_{\mathrm{as}}, \omega_{0}, \omega_{0},-\omega_{\mathrm{s}}\right) \\
& {\left[E_{1}\left(\omega_{0}\right)\right]^{2}\left[E_{1}\left(\omega_{\mathrm{s}}\right)\right]^{*}} \\
& \chi_{1111}^{(3)}=\frac{i N c^{4}}{12 \hbar \omega_{\mathrm{s}}^{4}} \frac{\partial \sigma}{\partial \Omega} S(\omega)+\chi^{\mathrm{NR}} \\
& S(\omega)=l^{\mathrm{t}}(G)^{-1} \Delta \rho_{0} l \\
& G=G(\omega)=i\left\{\omega I-\omega_{J}\right\}+N W \\
& \omega=\omega_{0}-\omega_{\mathrm{s}} \\
& \left(\omega_{J}\right)_{J J}=2 \pi c \nu(J) \delta_{J J} \\
& \left(\Delta \rho_{0}\right)_{J S}=\left[\rho_{\mathrm{eq}}(v=1, J)-\rho_{\mathrm{eq}}(v=0, J)\right] \delta_{J J} \\
& \frac{\partial \sigma}{\partial \Omega}=\frac{\omega_{s}^{4}}{c^{4}}\left|\sum_{r}\left\{\frac{\mu_{f r} \mu_{r i}}{\hbar\left(\omega_{r i}-\omega_{0}\right)}+\frac{\mu_{f r} \mu_{r i}}{\hbar\left(\omega_{f r}+\omega_{0}\right)}\right\}\right|^{2}
\end{aligned}
$$

with $N$ the number density, $l$ a column vector of 1 's (superscript " $t$ " signifying transpose), $I$ the unit matrix, $\omega_{J}$ and $\Delta \rho_{0}$ diagonal matrices of transition frequencies and (equilibrium) population differences respectively. A nonresonant contribution, $\chi^{\mathrm{NR}}$, to the third order susceptibility (see below) is included in eq (9). Equation (10) is a short-hand matrix equation for the frequency dependence of the (Raman) resonant part of the susceptibility which involves the relaxation matrix $W$. This matrix carries all the information about the effects of collisions on the spectrum; we will discuss it later.

\subsection{Raman Cross-Section}

First we note that eq (15) is an approximate expression for the Raman scattering cross-section. It explicitly shows the two, one-photon dipole matrix elements, $\mu_{\mathrm{mn}}$, connecting the initial $(i=\{v=0, J\})$ and final $(f=\{v=1, J\})$ molecular levels via intermediate electronic states, $r$ [the subscripts $\{m n\}$ stand for sets such as $\{r i\}$ or $\{f r\}$ in eq (15)]. This form of the cross-section is used because all photons (laser, Stokes, and, anti-Stokes) satisfy the relation

$$
\omega_{\mathrm{s}}<\omega_{0}<\omega_{\mathrm{as}} \ll \omega_{\mathrm{mn}}
$$

with

$$
\omega_{m n} \equiv \hbar^{-1}[E(m)-E(n)] .
$$


As a consequence of these conditions, no denominator in eq (15) is near zero, i.e., we are far from any one photon resonance.

The quantity inside the squared-magnitude symbols in eq (15) is an expression for the optical polarizability matrix element for the transition $\{i f\}$. The optical polarizability is a 2 nd-rank tensor quantity which, for the systems of interest here, has two irreducible tensor components. These are a scalar, rotationally invariant part of rank zero, and a traceless, symmetric 2nd-rank tensor part, known as the anisotropy. Reference to the "isotropic $Q$ branch" indicates consideration of that part of the interaction associated with the scaler part of the polarizability matrix elements.

The squared-magnitude of the matrix element of the scaler portion of the polarizability is linearly dependent on the vibrational quantum number but essentially independent of the rotational quantum number [6]. As a result, the contribution of an individual $Q(J)$ transition to the isotropic $Q$ branch is almost exactly proportional to the population difference in eq (14).

\subsection{Nonresonant Contribution to $\chi$}

Reference to eq (11) will show that the contribution of the Raman resonance to the susceptibility has both real and imaginary components. Far off the Raman resonance, when $\left|\omega-\omega_{J}\right| \gg\left|N W_{S J}\right|$ for all $\left\{J^{\prime}, J\right\}$, this contribution becomes pure real, cf. eq (9). When one includes all the possible field combinations implicit in eq (1), there are a large variety of far off-resonance two-photon transitions which add together to provide a constant-in-frequency real susceptibility called the nonresonant background. This is the source of $\chi^{\mathrm{NR}}$ in eq (9).

\subsection{CARS Intensity}

The polarization density in eq (8) is a source term in Maxwell's equations which are solved to yield the anti-Stokes field. For copropagating (along $z$ ), plane wave, linearly polarized (aiong $x$ ) pump and Stokes excitation, the approximate solution is a plane, anti-Stokes wave, with electric field parallel to $x(x \equiv 1)$

$$
\begin{aligned}
& E_{x}^{\mathrm{as}}=\mathrm{E}\left(\omega_{\mathrm{as}}, z\right) \exp \left(k_{\mathrm{as}} z-\omega_{\mathrm{as}} t\right) \\
& E\left(\omega_{\mathrm{as}} z\right)=\frac{i \pi \omega_{\mathrm{as}}}{2 c n_{\mathrm{as}}} \chi^{\mathrm{CARS}}\left[E\left(\omega_{0}\right)\right]^{2}\left[E\left(\omega_{\mathrm{s}}\right)\right]^{*} \\
& \frac{\sin (\delta k z / 2)}{\delta k / 2} \mathrm{e}^{i \delta k z / 2}
\end{aligned}
$$

with

$$
\chi^{\mathrm{CARS}}=12 \chi_{1111}
$$

and with

$$
\delta k=K_{\mathrm{as}}-k_{\mathrm{as}}
$$

expressing the phase matching condition. The wave vectors on the RHS of eq (21) are those of the CARS polarization, eq (4b), and the generated. radiative CARS field, eq (18). The intensity (power density) associated with this field is

$$
I\left(\omega_{a s}\right)=\frac{16 \pi^{4} \omega_{a s}^{2} I\left(\omega_{s}\right)\left[I\left(\omega_{0}\right)\right]^{2} \mid \chi^{C A R S p}}{c^{4} n_{0}^{2} n_{s} n_{\alpha s}}\left(\frac{\sin (\delta k / 2)}{\delta k / 2}\right)^{2} .
$$

Equations (18)-(22) involve some idealizations and approximations, such as plane wave excitation and weak field limits, which can easily be removed in the theory or to some extent achieved in the laboratory, or both, see for example reference [3]. These equations do provide a basis for understanding the essential features of the signal generation process, namely, an intensity proportional to: the squared magnitude of a third order nonlinear susceptibility, $\chi\left(-\omega_{a s}, \omega_{0}, \omega_{0},-\omega_{5}\right)$, the square of the pump intensity, the probe intensity, and a phase matching factor.

\subsection{Spatial Resolution and Phase Matching}

For systems with $\delta k \simeq 0$, e.g., low density gases, spatial resolution and signal enhancement is achieved simultaneously in the copropagating geometry, discussed above, by using focussed pump and Stokes lasers. Gaussian transverse-mode lasers yield nearly Gaussian anti-Stokes beams generated from spatial volumes of diameter less than the pump focal diameter and with axial distances of the order of $6 z_{0}$, with $z_{0}$ the familiar confocal parameter of the focussed pump beam [3]. For a 5 -mm diameter $\left(d_{0}\right)$ pump focussed by a $20-\mathrm{cm}(f)$ focussing lens. the approximate dimensions of the sample volume are found from the equations for the focussed beam diameter, $2 w_{0}$,

$$
2 w_{0} \simeq \frac{4 \lambda_{0}}{\pi} \frac{f}{d_{0}}=28 \times 10^{-4} \mathrm{~cm}
$$

and for the confocal parameter

$$
z_{0}=\frac{\pi w_{0}^{2}}{\lambda_{0}}=0.108 \mathrm{~cm}
$$


Spatial resolution can be enhanced by crossing the pump and Stokes beams, for example as shown in figure 1 . The requirements of phase matching for signal enhancement, c.f. eqs (21) and (22), result in the folded BOXCARS [11] arrangement shown in the figure. This arrangement will yield a spatial resolution nearer to the dimensions $2 w_{0}$ and $2 z_{0}$. Since the folded BOXCARS geometry yields good spatial separation of the anti-Stokes beam, this usually is the spectroscopically preferred arrangement. However, many other configurations are possible [2].

\subsection{CARS Spectrum}

The spectrum results from the frequency dependence of the squared magnitude of $\chi$. From eqs ( 9 ) and (10), we can write the general form

$$
|\chi|^{2}=\left(\chi^{\mathrm{NR}}\right)^{2}+2 \chi_{\mathrm{R}}^{\prime} \chi^{\mathrm{NR}}+\chi_{\mathrm{R}}^{\prime 2}+\chi_{\mathrm{R}}^{\prime \prime 2}
$$

with the subscript $\mathrm{R}$ referring to the (Raman) resonant part of the susceptibility and the prime denoting real and the double prime denoting imaginary part, respectively. If we limit consideration to a single, presumed isolated (our specific meaning for this term will be clarified below) transition, at $\omega_{J}$, the resonant part of the susceptibility is

$$
\chi_{\mathrm{R}} \propto \frac{\left(\omega-\omega_{J}\right)+i \Gamma_{J}}{\left(\omega-\omega_{J}\right)^{2}+\Gamma_{J}^{2}}
$$

with $\Gamma_{J}$ the linewidth of the pressure broadened line. The sum of the last two terms in eq (25) yields a Lorentzian lineshape, i.e.,

$$
I(\omega) \propto \frac{1}{\left(\omega-\omega_{J}\right)^{2}+\Gamma_{J}^{2}} .
$$

The presence of a nonzero nonresonant background leads to asymmetric lineshapes via the second term in eq (25). The first term yields a uniform nonzero signal level which can be dominant if the concentration of the resonant species is low.

\subsection{Collisional Line Interference}

A transition can be considered isolated if the condition expressed by the relation

$$
\sum_{J \neq J} \frac{W_{J J}}{\left(\omega_{J}-\omega_{J}\right)} \ll 1
$$

is satisfied. The quantity $W_{J J}$ is an off-diagonal ele- ment of the relaxation matrix introduced in eq (11). It is a measure of the rate of collisionally-induced exchange between transitions (see below). Equation (28) simply states that if the rate of exchange between transitions is much smaller than the frequency separation of the transitions, then they can be considered isolated. Conversely, if collisions are mixing lines at a rate commensurate with their separations, we will see line interferences. These are described by the full relaxation matrix equation. The corrections for multiple line spectra which arise when we cannot ignore the nondiagonality of the relaxation matrix in eq (11) are discussed in the following.

\subsection{The Relaxation Matrix}

10.12.1 Pressure Shift and Broadening In general, the diagonal elements of the relaxation matrix describe the familiar pressure broadening and shifting of spectral lines [12]. Thus, the resonant frequency and linewidth in eq (26) are

$$
\omega_{J}=\Omega_{J}+\delta_{J} P
$$

[with the convention that the quantity $\Omega_{J}$ is the zero pressure value derived from accurately known molecular constants, see for example eq (7)] and

$$
\Gamma_{J}=\gamma_{J} P
$$

Equations (29) and (30) implicitly define pressure shift and pressure broadening coefficients, $\delta$ and $\gamma$ respectively.

The change and spread of the observed resonance frequency are associated with intermolecular collisions. Encounters with other molecules alter the binding forces and therefore the resonance frequencies of the optically active molecule. In the pressure regimes of our interest, such encounters are limited to binary collision events such that the duration of the encounter is much shorter than the time between collisions. In simplest terms, the shifting coefficient expresses the average rate of collision-induced phase shift of the oscillator. Similarly, the broadening coefficient expresses the rate at which the oscillator is dephased by the collisions.

These effects are expressed as proportional to pressure because the probability per unit time that a collision produces a phase shift in the observed oscillation frequency can be written as the product $N \cdot v_{\mathrm{av}} \cdot \sigma$ of the number density, $N$ (molecule $/ \mathrm{cm}^{3}$ ), the average velocity, $v_{\mathrm{av}}(\mathrm{cm} / \mathrm{s})$, and a cross- 
section, $\sigma\left(\mathrm{cm}^{2}\right)$, appropriate to shifting or broadening for the collision. Such an expression is analogous to that for the collisional momentum transfer which defines the pressure in the gas.

We now turn to consideration of the off-diagonal elements of the relaxation matrix.

10.12.2 Line Mixing and State-to-State Rates In the familiar "impact limit" of collision physics (aspects of which were enumerated in the preceding section, see also ref. [12]), the elements of $W$ are proportional to appropriately averaged products of two scattering ("S-matrix") operators. For molecules such as $\mathrm{N}_{2}$ and $\mathrm{CO}$, it has been shown that there is very weak dependence of the scattering matrix elements on the vibrational states. This reveals itself in very weak shifting and a line broadening which is independent of vibrational (e.g., $1 \rightarrow 2$ vs $0 \rightarrow 1$ ) or overtone (e.g., $0 \rightarrow 2$ vs $0 \rightarrow 1$ ) branch. The absence of strong vibrational state dependencies in the $S$-matrix elements yields a relaxation matrix for the isotropic $Q$ branch which can be expressed entirely in terms of state-to-state rates for rotational energy transfer. The off-diagonal element, $W_{J J}$ for these conditions, is simply the negative of the inelastic rate for a transition from $J$ to $J^{\prime}$. In terms of the discussion in the last section, we can see that the occurrence of an inelastic collision terminates the oscillation, i.e., equivalently totally dephases the oscillator. Very little shifting would be expected under these conditions. Thus, the diagonal term, the line broadening coefficient, reduces to the total inelastic rate out of a state $J$.

Because of the nonzero off-diagonal components, the spectrum resulting from the relaxation matrix equation will show the phenomenon of collisional narrowing of the $Q$ branch with increasing pressure. The important criterion for the narrowing is a rapid rate of rotational energy transfer relative to the frequency difference between the $Q(J)$ transitions, i.e., the relation in eq (28) is not satisfied. Systems such as $\mathrm{N}_{2}$ and $\mathrm{CO}$ show significant narrowing at $1 \mathrm{~atm}$ and room temperature, because they have relatively small rotational energy gaps with concomitantly large rates of rotational energy transfer, and small interline spacings within the $Q$ branch. In contrast, the spectra of $\mathrm{D}_{2}$ displayed in figure 4 can be considered isolated, independent lines. We illustrate the effects of interference in the following.

\section{$10.13 Q$-Branch Spectra of $\mathrm{N}_{2}$}

Pressure dependent spectra of pure $\mathrm{N}_{2}$ at room $T$ are shown in figure 5. For comparison, an isolated line model $\left(W_{S J}=0\right.$ for $\left.J^{\prime} \neq J\right)$ for the spectrum at $10 \mathrm{~atm}$ is also included in the figure. Clearly, neglect of the collisional interference terms, $W_{s J}$, would lead to large errors in pressure determinations. The accuracy of theoretically predicted spectra, such as those shown in figure 5 , has been tested against spectra measured on samples in the pressure range up to $100 \mathrm{~atm}$ and for temperatures from 295 up to $2400 \mathrm{~K}$ (the upper limit in $T$ depends on $P$ because of the pressure limits of high- $T$ furnaces and cells), see reference [4].

\subsection{Energy Gap Laws for Rotational Energy Transfer}

The fundamental basis for calculation of the spectra shown in figure 5 is an energy-gap rate law [13] for the individual state-to-state rates for rotational energy transfer. As discussed above, knowledge of the state-to-state rates allows specification of the entire relaxation matrix and therefore

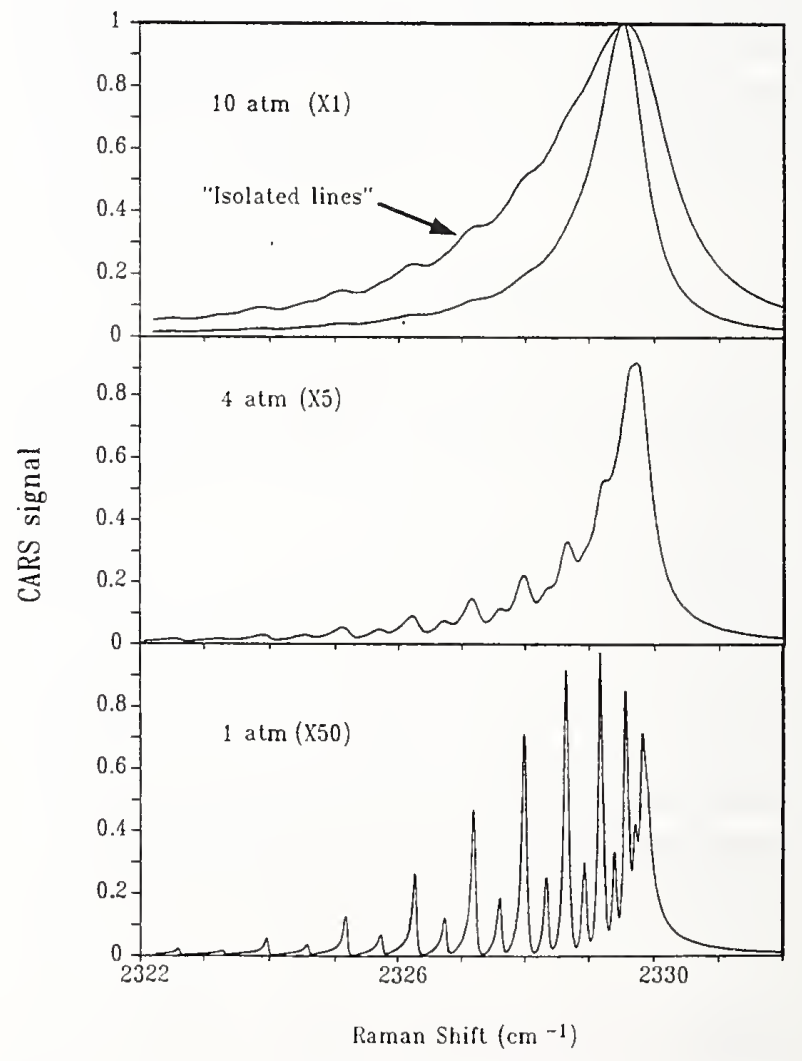

Figure 5. Calculated CARS spectra for the pure $\mathrm{N}_{2} Q$ branch at $295 \mathrm{~K}$. The intensity at each pressure has been multiplied by the indicated factor and plotted on a unit-normalized scale. An isolated line model which considers only the diagonal elements of the relaxation matrix is included for comparison at $10 \mathrm{~atm}$. 
computation of the spectrum [from eqs (9)-(11) and (22)]. The most widely used form of the rate law for specification of the relaxation matrix is the modified exponential gap (MEG) law [14] which parameterizes the upward $\left(J \rightarrow J^{\prime}\right.$ with $\left.J<J^{\prime}\right)$ stateto-state rate according to

$$
\begin{aligned}
& W_{J J}=\alpha_{0} f(T) \cdot\left(\frac{1+A E\left(J^{\prime}\right) / k T \delta^{\prime}}{1+A E\left(J^{\prime}\right) / k T}\right)^{2} \\
& \cdot\left(\frac{1+A E(J) / k T \delta}{1+A E(J) / k T}\right)^{2} \cdot \exp \left(\frac{-\beta\left\{E\left(J^{\prime}\right)-E(J)\right\}}{k T}\right)
\end{aligned}
$$

with

$$
f(T)=\left(\frac{T_{0}}{T}\right)^{1 / 2} \frac{1-\exp (-m)}{1-\exp \left(-m T / T_{0}\right)} .
$$

The downward rate is found from the principle of detailed balance, i.e.,

$$
\rho_{\mathrm{eq}}(J) W_{J \mathrm{~J}}=\rho_{\mathrm{eq}}\left(J^{\prime}\right) W_{J J}
$$

The quantity $A$ is calculated from the LennardJones intermolecular potential parameters for each molecular collision system. The parameters $\alpha_{0}, \delta^{\prime}$, $\delta, \beta$, and $m$ are determined from comparisons to accurately measured line broadening coefficients, $\gamma_{J}$, (functions of $J$ and $T$ ) and to experimentally determined pressure dependent spectra.

There remain some significant research issues in the prediction of $\mathrm{N}_{2}$ isotropic $Q$-branch spectra, such as: some refinement of the rate law model seems important at high temperature [15] and the appropriate spectral and rate law model for situations where $\mathrm{N}_{2}$ is found in a foreign gas host must be experimentally examined.

\subsection{Q-Branch Spectra of the Hydrogens}

The state-of-the-art for prediction of the spectrum of molecular systems such as $\mathrm{D}_{2}\left(\right.$ or $\left.\mathrm{H}_{2}\right)$ : $\mathrm{X}$ $\left[\mathrm{X}=\mathrm{D}_{2}\right.$ (or $\left.\left.\mathrm{H}_{2}\right), \mathrm{He}, \mathrm{Ar}, \mathrm{N}_{2}\right]$ is not as fully developed as for $\mathrm{N}_{2}: \mathrm{N}_{2}$. This situation arises because there is a large vibrational state dependence in the $S$-matrix elements. Such a vibrational state dependence leads to the addition of a significant "elastic vibrational dephasing," discussed earlier, contribution to the linewidth and line shift in these systems. The elastic vibrational dephasing component, although understood formally from theory, is not easily calculated and does not have a simple predictive basis similar to that just presented for the rotationally inelastic component.
The significance of elastic dephasing for the hydrogens is judged relative to their rotationally inelastic contributions. For the hydrogenic systems, the presence of elastic vibrational dephasing is evidenced by pressure shifting coefficients, $\delta$, [cf. eq (29)], which are of the order of and often a few times larger than the line broadening coefficients, $\gamma_{J}$ [cf. eq (30)]. Because of the large rotational energy gaps in these systems the inelastic portion of the total line broadening is relatively weak (especially for the foreign gas collision partners). Small inelastic rates and large vibrational dephasing both lead to a decrease in the line interference resulting from the solution of eqs (10) and (11). Finally, there is large rotation-vibration interaction in the hydrogens, e.g., $\alpha_{\mathrm{e}}$ in eq (7) is 1.08 for $D_{2}$, more than 60 times larger than the $\alpha_{e}$ of $N_{2}$. As a result of all these factors, the hydrogens will display essentially isolated-line type spectra with shift and width measurements yielding good measures of the pressure. The spectra displayed in figure 4 illustrate the pressure sensitivity of the pure gas system, those in figure 6 indicate the potential sensitivity for the $D_{2}$ :Ar system. The spectra given in this figure are based on actual measurements which to this point have been extended only to $50 \mathrm{~atm}$. Realizing the potential of this system requires more study as we will discuss in the following.

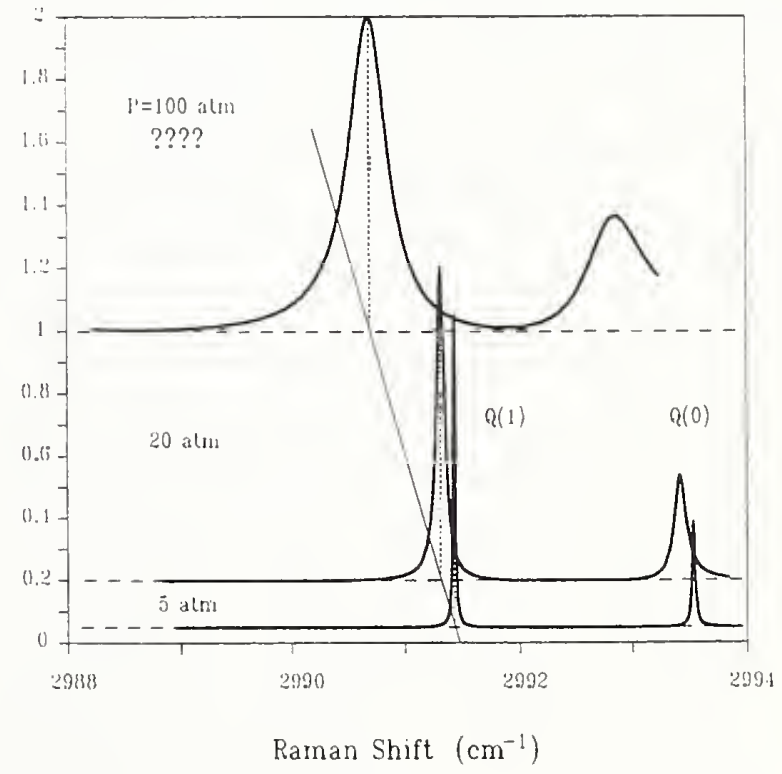

Figure 6. Calculated CARS spectra for the $J=0$ and $J=1$ transitions of $\mathrm{D}_{2}$ contained at $10 \%$ concentration in $\mathrm{Ar}$ at $295 \mathrm{~K}$. The spectra are based on experimental measurements which extend only up to $50 \mathrm{~atm}$. The extrapolation to $100 \mathrm{~atm}$ is based on the lower pressure data. See caption and compare to figure 4 . 
The complete $J, P$, and $T$ dependence of the spectra of $\mathrm{D}_{2}\left(\mathrm{H}_{2}\right)$ :X is yet to be determined. Some preliminary data on pure $\mathrm{H}_{2}$ up to $1000 \mathrm{~K}$ and 50 atm has been obtained. An empirical fitting law based on the MEG law for the rotationally inelastic contribution and a simple power law in $T$ for scaling the (assumed $J$ independent) vibrational dephasing contribution has been applied with some success to these data. More work is needed for all the systems of interest, in order to have a predictive basis.

In particular there remain some important scientific questions with respect to the foreign gas systems. Recent work has revealed anomalous concentration dependent lineshapes for the $\mathrm{D}_{2}$ (and $\mathrm{H}_{2}$ ):Ar system [8]. These anomalies are associated with a large (collision) speed dependence in the cross-sections for line shifting and a small propensity of $\mathrm{D}_{2}$ :Ar collisions to result in speed changes. As a result, the lines are inhomogeneously broadened and rather asymmetric at very low concentrations of the active molecule in the Ar host. More study of the concentration, pressure, and temperature dependence of the $\mathrm{D}_{2}$ :Ar lineshape is required.

\subsection{Doppler Broadening}

The line broadening, shifting, and interferences discussed above are appropriate to pressure and temperature regimes in which the rate of velocity changing collisions, $N v_{\mathrm{av}} \sigma_{\mathrm{D}}$, with $\sigma_{\mathrm{D}}$ the "diffusion cross-section," is larger than the Doppler width, $\approx\left|k_{0}-k_{\mathrm{s}}\right| v_{\mathrm{av}}$, of the Raman transition. Doppler broadening arises from the free streaming of the molecules, and, at low pressures, it can be used for measurement of translational temperature. For the hydrogens, in particular, this source of line broadening is significant at pressures up to $10 \mathrm{~atm}$ at high temperatures, e.g., $1000 \mathrm{~K}$. Research on the $\mathrm{D}_{2}: \mathrm{X}$ systems $\left(\mathrm{X}=\mathrm{D}_{2}, \mathrm{H}_{2}, \mathrm{He}, \mathrm{CH}_{4}, \mathrm{Ar}\right)$, under conditions where both velocity changing and normal pressure broadening collisions, as discussed above, contribute to the line shape is in progress. A reliable theoretical description appears possible, especially for relatively light collision partners, using a simple diffusion-like model solution to kinetic equations for line formation. However, more work, in particular for the heavy collision partners and high temperatures, remains to be done for these systems.

\subsection{Summary}

In the above, we have briefly summarized essential aspects of the theory of nonlinear Raman spec- troscopy as seen from the viewpoint of one who wishes to apply the technique to temperature and pressure measurements. This has involved some discussion of elements of the theory of spectral line formation for the relevant Raman transitions. Although the level of detail and rigor of this discussion has been limited, the intention is simply to indicate that we have a good understanding of the basic physics which underlies our measurement approach. A great deal of work has gone into building this understanding and developing a reliable predictive basis for temperature and pressure measurements. It is now possible to transfer the static pressure and temperature scale to the molecular level to serve as a primary standard for dynamic $P$ and $T$.

About the authors: Gregory J. Rosasco, Vern E. Bean, and Wilbur S. Hurst are physicists in the Temperature and Pressure Division of the NIST Center for Chemical Technology.

\section{References}

[1] Schweppe, J. L., Eichberger, L. C., Muster, D. F., Michaels, E. L., and Paskusz, G. F., Methods for the Dynamic Pressure Calibration of Transducers, National Bureau of Standards Monograph 67, (Government Printing Office, Washington, DC), 1963.

[2] Laser optical diagnostics and nonlinear Raman diagnostics are reviewed in:

Bechtel, J. H., Dash, C. J., and Teets, R. E., "Combustion research with lasers", in Laser Applications, Vol. 5, Ready, J. F., and Erf, R. K., eds., (Academic Press, Inc.), 1984.

Hall, R. J., and Eckbreth, A. C., "Coherent anti-Stokes Raman spectroscopy (CARS): application to combustion diagnostics", in Laser Applications, Vol. 5, Ready, J. F., and Erf, R. K., eds., (Academic Press, Inc.), 1984.

Greenhalgh, D. A., "Quantitative CARS spectroscopy", in Advances in Non-linear Spectroscopy, Clark, R. J. H., and Hester, R. E., eds., (John Wiley and Sons, Ltd), 1988.

Eckbreth, A. C., Laser Diagnostics for Combustion Temperature and Species, (Abacus Press, Cambridge, MA), 1988.

[3] Coherent anti-Stokes Raman spectroscopy is discussed in many recent articles and books, for example:

Druet, S. A. J., and Taran, J.-P., Prog. Quant. Electr. 7, 1 (1981).

Nibler, J. W., and Pubanz, G. A., "Coherent Raman spectroscopy of gases", in Advances in Non-linear Spectroscopy, Clark, R. J. H., and Hester, R. E., eds., (John Wiley and Sons, Ltd), 1988.

Chemical Applications of Nonlinear Raman Spectroscopy, Harvey, A. B., ed. (Academic Press, NY) 1981.

Eesley, G. L., Coherent Raman Spectroscopy, (Pergamon, Oxford), 1981. 
[4] The spectra of simple diatomic molecules such as $\mathrm{N}_{2}$ and $\mathrm{CO}$ have been studied by a number of groups, see for example:

Rosasco, G. J., Lempert, W., Hurst, W. S., and Fein, A., Chem. Phys. Lett. 97, 435 (1983).

Lavorel, B., Millot, G., Saint-Loup, R., Wenger, C., Berger, H., Sala, J. P., Bonamy, J., and Robert, D., J. Phys. (Paris) 47, 417 (1986).

Rahn, L. A., and Palmer, R. E., J. Opt. Soc. Am. B 3, 1164 (1986).

Koszykowski, M. L., Rahn, L. A., Palmer, R. E., and Coltrin, M. E., J. Phys. Chem. 91, 41 (1987).

Rahn, L. A., Palmer, R. E., Koszykowski, M. L., and Greenhalgh, D. A., Chem. Phys. Lett. 133, 513 (1987).

Rosasco, G. J., Rahn, L. A., Hurst, W. S., Palmer, R. E., Looney, J. P., and Hahn, J. W., Proc. SPIE 912, 171 (1988).

Rosasco, G. J., Rahn, L. A., Hurst, W. S., Palmer, R. E., and Dohne, S. M., J. Chem. Phys. 90, 4059 (1989).

Looney, J. P., Rosasco, G. J., Rahn, L. A., Hurst, W. S., and Hahn, J. W., Chem. Phys. Lett. 161, 232 (1989).

The data base for the hydrogens is more limited with regard to the range of temperature (especially above room temperature) and pressure for which accurate measurements have been reported. Summaries of the applicable measurements are contained in:

Rosasco, G. J., and Hurst, W. S., "The effects of velocity and phase changing collisions on Raman $Q$-branch spectra", in Spectral Line Shapes, Vol. 4, Exton, R. J., ed., (A. Deepak Publishing, Virginia), 1987.

Smyth, K. C., Rosasco, G. J., and Hurst, W. S., J. Chem. Phys. 87, 1001 (1987).

Rosasco, G. J., May, A. D., Hurst, W. S., Petway, L. B., and Smyth, K. C., J. Chem. Phys. 90, 2115 (1989).

Farrow, R. L., Rahn, L. A., Sitz, G. O., and Rosasco, G. J., Phys. Rev. Lett. 63, 746 (1989).

Rahn, L. A., and Rosasco, G. J., submitted to Phys. Rev. A.

[5] Raman spectroscopy is reviewed for diatomic molecules and general gas phase systems in:

Herzberg, G., Molecular Spectra and Molecular Structure I. Spectra of Diatomic Molecules, (D. Van Nostrand Co. Inc.) 1950.

Weber, A., "High resolution Raman studies of gases", in The Raman Effect, Vol. 2, Anderson, A., ed., (Marcel Decker, NY), 1973.
[6] Intensity correction factors for the $Q$ branch are given in: James, T. C., and Klemperer, W. J., Chem. Phys. 31, 130 (1959).

[7] Recent reports of the use of pure rotational CARS spectra for temperature determinations are given in:

Eckbreth, A. C., and Anderson, T. J., Opt. Lett. 11, 496 (1986).

Alden, M., Bengtsson, P.-E., and Edner, H., Appl. Opt. 25, 4493 (1986).

[8] Farrow, R. L., Rahn, L. A., Sitz, G. O., and Rosasco, G. J., Phys. Rev. Lett. 63, 746 (1989).

[9] In addition to the references in [3], general references on nonlinear optics include:

Yariv, A., Quantum Electronics, Second Edition, (John Wiley and Sons, New York, 1975), Chapters 16 and 18.

Levenson, M. D., Introduction to Nonlinear Laser Spectroscopy, (Academic Press, New York, 1982), Chapter 4.

Shen, Y. R., The Principles of Nonlinear Optics, (John Wiley and Sons, New York, 1984).

[10] The formulae in eqs (8)-(15) are adapted from reference [3].

[11] Shirley, J. A., Hall, R. J., and Eckbreth, A. C., Opt. Lett. 5, 380 (1980).

[12] Excellent reviews of the fundamentals of spectral line broadening are given in:

Sobel'man, I. I., Introduction to the Theory of Atomic Spectra, (Pergamon Press, Oxford, 1972), Chapter 10.

Fiutak, J., and Van Kranendonk, J., Can. J. Phys. 40, 1085 (1962); 41, 21 (1963).

Ben Reuven, A., Adv. Chem. Phys. 33, 235 (1975).

[13] Brunner, T. A., and Pritchard, D., Adv. Chem. Phys. 50, 589 (1982).

[14] Koszykowski, M. L., Rahn, L. A., Palmer, R. E., and Coltrin, M. E., J. Phys. Chem. 91, 41 (1987).

[15] Porter, F. M., Greenhalgh, D. A., Williams, D. R., Baker, C. A., Woyde, M., and Stricker, W., Proceedings of the XIth International Conference on Raman Spectroscopy, London, 1988.

Rahn, L. A., Palmer, R. E., Koszykowski, M. L., and Greenhalgh, D. A., Chem. Phys. Lett. 133, 513 (1987).

Looney, J. P., Rosasco, G. J., Rahn, L. A., Hurst, W. S., and Hahn, J. W., Chem. Phys. Lett. 161, 232 (1989). 


\section{WORKSHOP ON THE MEASUREMENT OF TRANSIENT \\ PRESSURE AND TEMPERATURE \\ April 23-24, 1991 \\ Final Participants List}

Kee Abe11

NAVSWC

Dahlgren Lab.

Code E 13A

Dahlgren, VA 22448-5000

John Bal1

U.S. Army TMDE Support Group

AMXTM - DT

Redstone Arsenal, AL 35898-5400

Vern Bean

NIST

Bldg 221, Rm. B126

Gaithersburg, MD 20899

Donald Bornemann

EG\&G

P.O. Box 3000

Miamisburg, OH 45343

William Bryant

Edwards A.F.B.

6510 Test Wing TSIM

Edwards A.F.B., CA 93523-5000

Ared Cezairliyan

NIST

Bldg. 236, Rm. 124

Gaithersburg, MD 20899

Thomas Chandy

Endevco

30700 Rancho Viejo

San Juan Capistrano, CA 92675

Lang-Mann Chang

U.S. Army Ballistic Res. Lab.

Aberdeen Proving Ground

SLCBR-IB - P

Aberdeen, MD 21005

Hao-Yun Chou

C. Shan Inst. of Sci. \& Tech.

P.O. Box 90008-9-9

Lung-tan; Tawain, 32500

R.O.C.

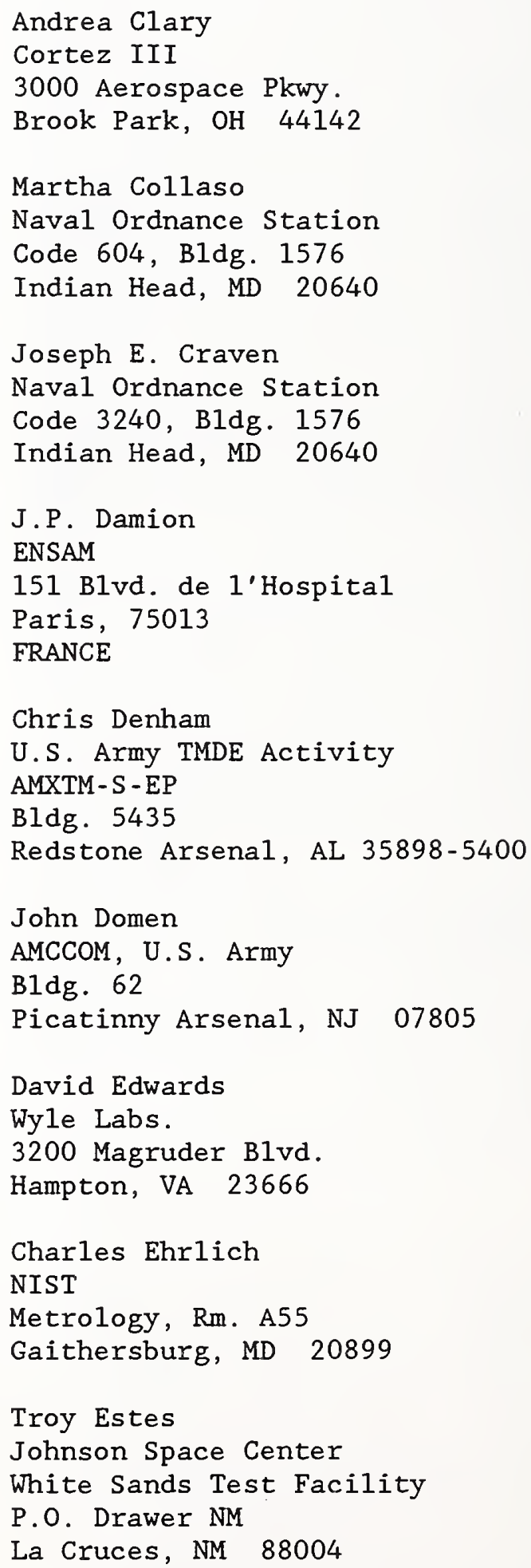


David Evans

NIST

Bldg. 233, Rm. A149

Gaithersburg, MD 20899

Douglas Evans

U.S. Air Force

WL/MNGI

Bldg. 13, Rm. 114

Eglin AFB, FL 32542

Timothy Fackler

Naval Surface Warfare Center

10901 New Hampshire Ave.

R15 B336-210

Silver Spring, MD 20903

Brian Fitzpatrick

$\mathrm{HI}-\mathrm{TECH}$, Inc.

8223 Cloverleaf $\mathrm{Dr}$.

Ste. $126 \mathrm{~A}$

Millersville, MD 21108

David Frank

Navy Primary Std.'s Lab.

Washington Navy Yard

Bldg. 219-2 Code 064

Washington, DC 20374

Charles Gerek

Naval Air Test Center

Patuxent River, MD 20670-5304

Bill Grahn

Edwards A.F.B.

6510 Test Wing TSIM

Edwards A.F.B., CA 93523-5000

Ben Granath

PCB Piezotronics

3425 Walden Ave.

Depew, NY 14043-2495

Robert Hall

General Physics Corp.

9891 Broken Land Pkwy.

Suite 400

Columbia, MD 21045

Bruce Hartenbaum

H-TECH Laboratories

P.O. Box 1686

Santa Monica, CA 90406
Marilyn Hartman

Navy Primary Std's Lab.

Washington Navy Yard

Bldg. 219-2 Code 064

Washington, DC 20374

Nat Hudson

Alliant Techsystems Inc.

7225 Northland Dr.

MS 48-2100

Brooklyn Park, MN 55428-1596

Wilbur Hurst

NIST

Bldg. 221/Rm. B126

Gaithersburg, MD 20899

Mark Hutchenson

Langley Research Center

MS 236

Hampton, VA 23665-5225

Willis F. Jackson

Applied Research Assoc.

30 Diamond St.

P.O. Box 548

Aberdeen, MD 21001

Dave Jaros

PCB Piezotronics

3425 Walden Ave.

Depew, NY 14043-2495

Robert Jobe

General Dynamics/Ft. Worth

P.0. Box 748

MZ 5838

Ft. Worth, TX 76101

Per-Ake Karlsson

Swedish Ordnance

Tullgatan 8

Eskilstuna, S-631 87,

Sweden

Don Keller

K-Tech

901 Pennsylvania Ave. NE

Albuquerque, NM 87110

Douglas Keough

SRI

333 Ravenswood Ave.

Menlo Park, CA 94025 
Ken Klingaman

U.S . Army ARDC

Bldg. 1501

Picatinny Arsenal, NJ 07806

John Kubler

Kistler Instruments

75 John Glenn Dr.

Amherst, NY 14228

Jim Lally

PCB Peizotronics

3425 Walden Ave.

Depew, NY 14043-2495

Ralph S. Lucas

Naval Ordnance Station

Code 604, Bldg. 1576

Indian Head, MD 20640

Dave Madden

U.S. Air Force, AGMC

Newark AFB, OH 43057-5475

Walter Markus

NIST

B1dg. 220, Rm. A53

Gaithersburg, MD 20899

Ray Massarotti

Wyle Labs

3200 Magruder Blvd.

Hampton, VA 23666

Ken McMullen

U.S. Army CSTA

STECS - EN - BI

Aberdeen, MD 21005

Richark Mersiowsky

Naval Surface Warfare Ctr.

R15

Dahlgren, VA 22448-5000

Bill Monette

Cummings Electronics

2851 State St.

Columbus, OH 47201

Donald H. Newhall

Harwood Engineering

455 South St.

Walpole, MA 02081-2799

\author{
Sten Orrling \\ Swedish Ordnance \\ Tullgatan 8 \\ Eskilstuna, S-631 87, \\ Sweden
}

Hugh Oslin

10901 Naval Surface Warfare Ctr.

New Hamphsire Ave.

4-238, Code E13A

Silver Spring, MD 20903-5000

Daniel Parobek

Air Force Wright Lab.

WL/FIMN

Wright Patterson AFB, OH 45433-6553

Philip Peregino

Ballistic Research Lab.

SLCBR - TB-EE

Aberdeen Proving Ground, MD 21005

Charlie Pugh

Gans and Pugh

690 Center St.

Herndon, VA 22070

Jeffrey Rauch

Sverdrup Technology

2001 Aerospace Pkwy.

Brookpark, OH 44142

Josef Riegebauer

AVL LIST Ges.m.b.H

$\mathrm{K}$ leiststrasse 48

Graz, A-8020, Austria

Gregory Rosasco

NIST

Bldg 221/Rm. B306

Gaithersburg, MD 20899

Arthur Rusche

Boeing

P.0. Box 3707

MS 47-08

Seattle, WA 98124

Kent Rye

Naval Surface Warfare Ctr.

10901 New Hampshire Ave.

R15

Silver Spring, MD 20903 
Jeff Schnellinger

Kistler Instruments

75 John Glenn Dr.

Amherst, NY 14228

Hratch Semerjian

NIST

Bldg. 221/Rm. B306

Gaithersburg, MD 20899

Robert Sill

Endevco

30700 Rancho Viejo Road

San Juan Capistrano, CA 92675

William C. Smith

LESC White Sands Test Fac.

P.O. Drawer MM

Rm. 201

Las Cruces, NM 88004

Eugene Stefko

Naval Ordnance Station

Code 604, Bldg. 1576

Indian Head, MD 20640

Peter K. Stein

Stein Engineering Service

5602 E. Monte Rosa

Phoenix, AZ 85018

Chang-Kun Tai

CMS/ITRI

321 Kuang-fu Road, Sec. 2

Hsinchu, Taiwan

R.O.C.

Chris Talebkhah

PCB Piezotronics

3425 Walden Ave.

Depew, NY 14043-2495

Dennis Taylor

Raytheon Service Co.

NSWC Dahlgren

Dahlgren, CA 22448-5000

John Terrell

AVL North America

4641 N. First Ave., 非3

P.O. Box 36438

Tucson, AZ 85718
Ronald B. Tussing

Naval Surface Warfare Ctr.

White Oak Lab.

MC 336-113

Silver Spring, MD 20903-5000

William P. Twigg

Westinghouse ESG

P.O. Box 746

Mail Stop T-29

Baltimore, MD 21203

Don Ward

NIST

Metrology Bldg./Room. A55

Gaitherburg, MD 20899

Kate Webster

Cortez III

3000 Aerospace Pkwy.

Brook Park, OH 44142

Janet Whipple

Langley Research Center

MS 234

Hampton, VA 23665-5225

Marek Winiarz

GE Aircraft Engines

10270 St. Rita La.

MD Q45

Cincinnati, $\mathrm{OH} \quad$ 45215-6301

Bill Wolber

Cummings Electronics

2851 State St.

Columbua, $\mathrm{OH} 47201$

Jerry W. Wood

Pratt \& Whitney

P.O. Box 109600

MS 716-87

West Palm Beach, FL 33410-9600

John Wright

NSWC/Dahlgren

R15

King George, VA 22448 



\begin{tabular}{|c|c|}
\hline \multirow[t]{2}{*}{$\begin{array}{l}\text { U.S. DEPARTMENT OF COMMERCE } \\
\text { NATIONAL INSTITUTE OF STANDARDS AND TECHNOLOGY }\end{array}$} & \begin{tabular}{|l|} 
1. PUBLICATION OR REPORT NUMBER \\
NISTIR 4828 \\
2. PERFORMING OROANIZATION REPORT MUMBER
\end{tabular} \\
\hline & $\begin{array}{l}\text { 3. PUBUCATION DATE } \\
\text { APRIL } 1992\end{array}$ \\
\hline \multicolumn{2}{|c|}{$\begin{array}{l}\text { 4. TITLEAND SUBTITLE } \\
\text { Executive Summary: Proceedings of the Workshop on the Measurement of Transient Pressure } \\
\text { and Temperature }\end{array}$} \\
\hline \multicolumn{2}{|l|}{$\begin{array}{l}\text { 5. AUTHOR(S) } \\
\text { Vern E. Bean and Gregory J. Rosasco }\end{array}$} \\
\hline $\begin{array}{l}\text { 6. PERFORMING ORGANIZATION (IF JOINT OR OTHER THAN MIST, SEE INSTRUCTIONS) } \\
\text { U.S. DEPARTMENT OF COMMEACE } \\
\text { MATIONAL INSTITUTE OF STANDARDS AND TECHNOLOGY } \\
\text { GATTHERSBURG, MD 20899 }\end{array}$ & $\begin{array}{l}\text { 7. CONTRACT/GRANT NUMBER } \\
\text { 8. TYPE OF REPORT AND PERIOD COVERED }\end{array}$ \\
\hline \multicolumn{2}{|l|}{$\begin{array}{l}\text { 9. SPONSORING ORQANIZATION MAME AND COMPLETE ADDRESS (STREET, CITY, STATE, ZIP) } \\
\text { National Institute of Standards and Technology } \\
\text { Gaithersburg, MD } 20899\end{array}$} \\
\hline
\end{tabular}

11. ABSTRACT (A 200-WORD OR LESS FACTUAL SUMMARY OF MOST SIGNIFICANT INFORMATION. IF DOCUMENT INCLUDES A SIGNIFICANT BIBLOGRAPHY OR LITERATURE SURVEY, MENTION IT HERE)

The talks and the discussions at the Workshop on the Measurement of Transient Pressure and Temperature held at NIST, Gaithersburg, MD on 23-24 April 1991 are reviewed. Twelve talks were presented describing measurement needs, the state-of-the-art in making these measurements, the need for establishing national standards for such measurements, and possible methods for improving these measurements.

12. KEY WORDS (6 TO 12 ENTRIES; ALPHABETICAL ORDER; CAPITAUZE ONLY PROPER NAMES; AND SEPARATE KEY WORDS BY SEMICOLONS) pressure, temperature, dynamic measurements, transient measurements, national standards

\section{AVAILABIUTY}

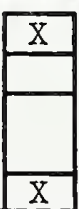

UNUMITED

FOR OFFICIAL DISTRIBUTION. DO NOT RELEASE TO MATIONAL TECHNICAL INFORMATION SERVCE (NTIS).

ORDER FROM SUPERINTENDENT OF DOCUMENTS, U.S. GOVERNMENT PRINTING OFFICE, WASHINGTON, DC 20402.

ORDER FROM NATIONAL TECHMICAL INFORMATION SERVCE (NTIS), SPRINGFIELD, VA 22161.
14. NUMBER OF PRINTED PAGES

218

15. PRICE

Al0 

